\title{
Extrapolação em espaços de Köthe
}

\author{
Víctor Juan Hernández Del Toro
}

\author{
DisSERTAÇÃO APRESENTADA \\ AO
Instituto de Matemática E Estatística \\ DA \\ Universidade de São Paulo \\ PARA \\ OBTENÇÃO DO TÍTULO \\ DE \\ Mestre em CiÊncias
}

Área de Concentração: Matemática
Orientador: Prof. Dr. Valentin Raphael Henri Ferenczi

Durante o desenvolvimento deste trabalho o autor recebeu auxílio financeiro da CNPq

São Paulo, novembro de 2014 


\section{Extrapolação em espaços de Köthe}

Esta versão da dissertação contém as correções e alterações sugeridas pela Comissão Julgadora durante a defesa da versão original do trabalho, realizada em 29/01/2015. Uma cópia da versão original está disponível no Instituto de Matemática e Estatística da Universidade de São Paulo.

Comissão Julgadora:

- Prof. Dr. Valentin Ferenczi (orientador) - IME-USP

- Prof. Dr. Eloi Medida Galego-IME-USP

- Prof. Dr. Jordi Lopez-Abad -ICMAT 


\section{Resumo}

\section{Extrapolação em espaços de Köthe}

Neste trabalho apresentamos alguns resultados da teoria de extrapolação desenvolvida por Nigel Kalton em [16].

Nós introduzimos o conceito de Soma torcida e apresentamos algumas das suas propriedades. Em seguida descrevemos um método de construção de somas torcidas ideado pelo Kalton e Peck para dar uma solução alternativa ao problema 3SP de espaços de Hilbert (ver [19]).

Nós estudamos um tipo de função $\Omega: X \longrightarrow L_{0}(\mu)$ definida em um espaço de Köthe chamada de Centralizador, que satisfaz:

- $\Omega(\alpha x)=\alpha \Omega(x)$ para todo $x \in X$ e todo $\alpha \in \mathbb{C}$.

- Existe uma constante $\rho(\Omega)>0$ tal que

$$
\|\Omega(u x)-u \Omega(x)\|_{X} \leq \rho(\Omega)\|u\|_{\infty}\|x\|_{X} .
$$

para todo $u \in L_{\infty}(\mu)$ e todo $x \in X$.

Além disso, se $\Omega(f)$ é uma função real sempre que $f$ seja uma função real, dizemos que $\Omega$ é um Centralizador real.

Seja $0<\theta<1$. Se $X=\left[X_{0}, X_{1}\right]_{\theta}$ é um espaço interpolado de Calderón (ver [1]), então definimos um Centralizador neste espaço, chamado de Centralizador induzido, por $\Omega_{0}(x)=$ $F^{\prime}(\theta)$, onde $F: \overline{\mathbb{S}} \longrightarrow X_{0}+X_{1}$ é uma função definida na faixa $\overline{\mathbb{S}}=\{z \in \mathbb{C}: 0 \leq \operatorname{Re}(z) \leq 1\}$ que satisfaz:

- $F$ é analítica no interior de $\overline{\mathbb{S}}$,

- $F(i t) \in X_{0}$ e $F(1+i t) \in X_{1}$ para todo $t \in \mathbb{R}$ e as funções $t \mapsto F(i t)$ e $t \mapsto F(1+i t)$ são contínuas. 
- $F$ verifica

$$
\|F\|_{\mathscr{F}}=\max \left(\sup _{t \in \mathbb{R}}\|F(i t)\|_{X_{0}}, \sup _{t \in \mathbb{R}}\|F(1+i t)\|_{X_{1}}\right)<\infty
$$

Logo, damos o conceito de função indicadora, que foi inicialmente introduzido por Gillespie (ver [25]) e usado pelo Kalton para resolver alguns problemas de extrapolação. Nós apresentamos as provas dos seguintes resultados:

Um de eles demonstrado pelo Pisier em [13]:

Se X é um espaço de Köthe p-convexo e q-côncavo, onde $\frac{1}{p}+\frac{1}{q}=1$, então para $\frac{\theta}{2}=\frac{1}{q} e$ $0<\theta<1$ existe um único espaço de Köthe $Y$ tal que

$$
X=L_{2}^{\theta} Y^{1-\theta}
$$

Nós vamos apresentar a prova deste teorema usando como ferramenta as funções indicadoras (prova de Kalton).

E outro resultado usando centralizadores:

Seja X um espaço de Köthe p-convexo e q-côncavo, onde $1 / p+1 / q=1$, com $1<p \leq 2$. Se $\Omega: X \longrightarrow L_{0}(\mu)$ é um centralizador real, com $\rho(\Omega) \leq 1 / 200 q$, então existem espaços de Köthe $X_{0}$ e $X_{1}$ tais que $X=\left[X_{0}, X_{1}\right]_{1 / 2}$ e se $\Omega_{0}$ é o centralizador induzido em $X$, então

$$
\left\|\Omega(x)-\Omega_{0}(x)\right\|_{X} \leq C\|x\|_{X}, \text { para todo } x \in X
$$

e alguma constante positiva $C$.

Palavras-chave: Espaço de Köthe, Espaço interpolado, Indicador de um espaço de Köthe , Centralizador. 


\section{Abstract}

\section{Extrapolation in Köthe spaces}

In this work we present some results in the Extrapolation Theory of Banach Spaces develop by Nigel Kalton in [16].

We introduce the concept of Twisted sums and we present some properties. we describe a method of construction of twisted sums develop by Kalton and Peck to give an alternate solution to the problem 3SP for Hilbert spaces (ver [19]).

We study a type of function $\Omega: X \longrightarrow L_{0}(\mu)$ defined on a Köthe space, called Centralizer, that satisfies:

- $\Omega(\alpha x)=\alpha \Omega(x)$ for all $x \in X$ and $\alpha \in \mathbb{C}$.

- There exists a constant $\rho(\Omega)>0$ such that

$$
\|\Omega(u x)-u \Omega(x)\|_{X} \leq \rho(\Omega)\|u\|_{\infty}\|x\|_{X} .
$$

for all $u \in L_{\infty}(\mu)$ and $x \in X$,

furthermore, if $\Omega(f)$ is a real function whenever $f$ is a real function, then $\Omega$ is called Real centralizer.

Let $0<\theta<1$. If $X=\left[X_{0}, X_{1}\right]_{\theta}$ is a Calderón interpolation space ( see [1] ), then we define a Centralizer in $X$, called Induced centralizer, by $\Omega_{0}(x)=F^{\prime}(\theta)$, where $F: \overline{\mathbb{S}} \longrightarrow X_{0}+X_{1}$ is a function defined on $\overline{\mathbb{S}}=\{z \in \mathbb{C}: 0 \leq \operatorname{Re}(z) \leq 1\}$, that satisfies:

- $F$ is analytic in the interior of $\overline{\mathbb{S}}$ and bounded on $\partial \overline{\mathbb{S}}$.

- $F(i t) \in X_{0}$ and $F(1+i t) \in X_{1}$ for all $t \in \mathbb{R}$ and $t \mapsto F(i t)$ and $t \mapsto F(1+i t)$ are continuous functions. 
- $F$ satisfies

$$
\|F\|_{\mathscr{F}}=\max \left(\sup _{t \in \mathbb{R}}\|F(i t)\|_{X_{0}}, \sup _{t \in \mathbb{R}}\|F(1+i t)\|_{X_{1}}\right)<\infty
$$

We introduce the concept of Indicator function, which was initially introduced by Gilliespie (ver [25]) and used by Kalton to solve some extrapolation problems. We present the proofs of the following theorems :

Pisier proved the following theorem (ver [13]):

Let $X$ be a Köthe space which isp-convex andq-concave where $1<p<2$ and $1 / p+1 / q=1$. Then there is a Köthe space $Y$ such that

$$
X=L_{2}^{\theta} Y^{1-\theta}
$$

where $1 / q=\theta / 2$.

We use indicator functions to prove this theorem (Kalton's proof).

Using centralizers, we proved the following extrapolation theorem:

Let $X$ be a Köthe space which isp-convex andq-concave where $1<p<2$ and $1 / p+1 / q=1$. If $\Omega$ is a real centralizer on $X$ with $\rho(\Omega) \leq 1 / 200 q$, then there is a pair of Köthe spaces $X_{0}, X_{1}$ such that $X=\left[X_{0}, X_{1}\right]_{1 / 2}$ and if $\Omega_{0}$ is the induced centralizer, then

$$
\left\|\Omega(x)-\Omega_{0}(x)\right\|_{X} \leq C\|x\|_{X}, \text { for all } x \in X
$$

and some $C>0$.

Keywords: Köthe space, Interpolation space, Indicator of a Köthe space, Centralizer. 


\section{Índice}

Índice $\quad v$

1 Introdução 1

2 Resultados preliminares

2.1 Teoria da medida $\ldots \ldots \ldots \ldots \ldots \ldots \ldots \ldots \ldots$

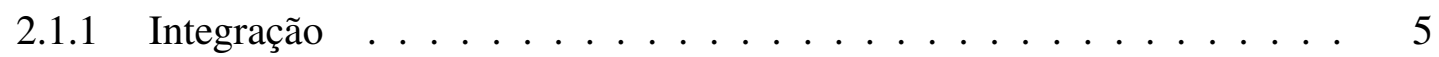

2.1.2 Tipos de convergências . . . . . . . . . . . . . . 8

2.1 .3 Integral de Bochner . . . . . . . . . . . . . . . . . . 9

2.2 Funções diferenciáveis . . . . . . . . . . . . . . . . . . . 10

2.2 .1 Funções analíticas . . . . . . . . . . . . . . . . . . 10

2.2.2 Funções de Lipschitz . . . . . . . . . . . . . . . . . . . . . . 11

2.3 Espaços de Banach reticulados . . . . . . . . . . . . . . . . . 11

2.3.1 $p$-potências de espaços de funções $\ldots \ldots \ldots \ldots \ldots$

2.3.2 Normas de ordem contínua . . . . . . . . . . . . . . . . . . . . . 13

2.3.3 Espaço de Banach de sequências (E.B.S) _ . . . . . . . . . . 13

2.4 Espaços quase-normados . . . . . . . . . . . . . . . . . . . . . . 14

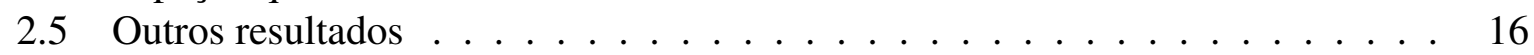

3 Somas torcidas 21

4 Interpolação em espaços de Banach $\quad 28$

4.1 Generalidades da teoria de interpolação . . . . . . . . . . . . . 28

4.2 Interpolação Complexa . . . . . . . . . . . . . . . . . . . . . . . . . 29

5 Espaços de Köthe e espaços de funções $g$-convexos 
$6 \quad O$ indicador de um espaço de funções $g$-convexo 62

6.1 Propriedades dos indicadores . . . . . . . . . . . . . . 63

6.2 Aproximação de funcionais semilineares . . . . . . . . . . . . . . . . . . 91

7 Centralizadores em espaços de Köthe 121

7.1 Propriedades dos centralizadores . . . . . . . . . . . . . . . . . 121

7.2 Auto-extensões de espaços interpolados . . . . . . . . . . . . . . . 127

8 Teoremas de extrapolação 137

$\begin{array}{ll}\text { Bibliografia } & 144\end{array}$ 


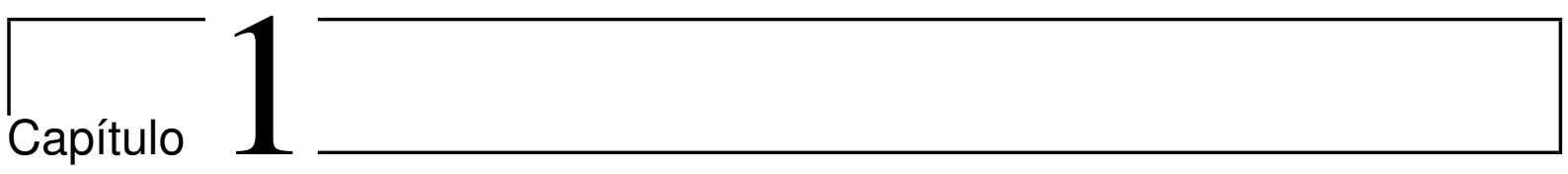

\section{Introdução}

Sabemos que uma auto-extensão de um espaço de Banach $Y$, é um espaço de Banach $X$ tal que $Y$ está mergulhado em $X$ e o espaço quociente $X / Y$ é isomorfo a $Y$ (ver [9]). O estudo das auto-extensões de um espaço nos conduz ao estudo das somas torcidas (ver Capítulo 3). Uma soma torcida $X$ de $Y$ e $Z$ tem associada uma sequência exata curta

$$
0 \rightarrow Y \stackrel{i}{\rightarrow} X \stackrel{j}{\rightarrow} Z \rightarrow 0 .
$$

Em [19], Kalton e Peck apresentam um método para construir somas torcidas de espaços de Banach $Y$ e $Z$. Eles consideram uma função $F: Z \longrightarrow W$, onde $W$ é um espaço vetorial com $Y \subset W$, que verifica

(i) F é homogênea,

(ii) existe $K$ positivo, tal que para todo todo $z_{1}, z_{2} \in Z$

$$
\left\|F\left(z_{1}+z_{2}\right)-F\left(z_{1}\right)-F\left(z_{2}\right)\right\|_{Y} \leq K\left(\left\|z_{1}\right\|_{Z}+\left\|z_{2}\right\|_{Z}\right)
$$

e demonstram que

$$
Z \oplus_{F} Y=\{(z, w) \in Z \times W: w-F(z) \in Y\}
$$

munido da quase-norma

$$
\|(z, w)\|_{Z \oplus_{F} Y}=\|z\|_{Z}+\|w-F(z)\|_{Y}
$$

é uma soma torcida de $Y$ e $Z$. Reciprocamente, Kalton e Peck demonstram que toda soma torcida $X$ de $Y$ e $Z$ é equivalente a uma soma torcida da forma $Z \oplus_{F} Y$ no seguinte sentido: existe um operador linear limitado $T: X \rightarrow Z \oplus_{F} Y$ tal que o seguinte diagrama comuta

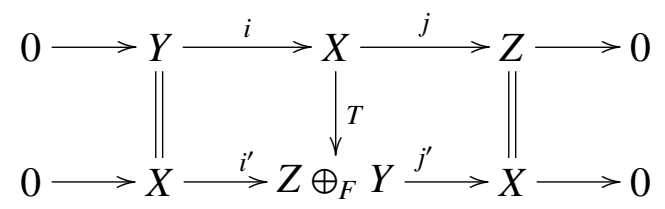


No caso $Y=Z$, denotamos $Y \oplus_{F} Y$ por $d_{F} Y$.

O Capítulo 4 é dedicado ao estudo do método de interpolação complexa desenvolvido por Calderón em [1].

Calderón usa o espaço $\mathscr{F}=\mathscr{F}\left(X_{0}, X_{1}\right)$ de funções analíticas no interior da faixa $\overline{\mathbb{S}}=\{z \in \mathbb{C}$ : $0 \leq \operatorname{Re}(z) \leq 1\}$ com valores em $X_{0}+X_{1}$ tais que as funções $t \mapsto F(i t), t \mapsto F(1+i t)$ são contínuas e

$$
\|F\|_{\mathscr{F}}=\max \left(\sup _{t \in \mathbb{R}}\|F(i t)\|_{X_{0}}, \sup _{t \in \mathbb{R}}\|F(1+i t)\|_{X_{1}}\right)<\infty,
$$

para definir o espaço intermédio entre $X_{0}$ e $X_{1}$ como

$$
\left[X_{0}, X_{1}\right]_{\theta}=X_{\theta}=\{F(\theta): F \in \mathscr{F}\}
$$

com norma

$$
\|x\|_{X_{\theta}}=\inf \left\{\|F\|_{\mathscr{F}}: F(\theta)=x\right\},
$$

onde $0<\theta<1$.

Quando ou $X_{0}$ ou $X_{1}$ são espaços reflexivos, o espaço $X_{\theta}$ pode-se fatorar da forma $X_{\theta}=X_{0}^{1-\theta} X_{1}^{\theta}$, onde $X_{0}^{1-\theta} X_{1}^{\theta}$ é definido por

$$
X_{0}^{1-\theta} X_{1}^{\theta}=\left\{x \in L_{0}: \text { existem } u_{0} \in X_{0}, u_{1} \in X_{1} \text {, tais que }|x|=\left|u_{0}\right|^{1-\theta}\left|u_{1}\right|^{\theta}\right\},
$$

munido da norma

$$
\|x\|=\inf \left\{\left\|u_{0}\right\|_{X_{0}}^{1-\theta} \cdot\left\|u_{1}\right\|_{X_{1}}^{\theta}:|x|=\left|u_{0}\right|^{1-\theta}\left|u_{1}\right|^{\theta}\right\} .
$$

Nós sempre vamos supor que $X_{\theta}=X_{0}^{1-\theta} X_{1}^{\theta}$.

No Capítulo 5 damos os conceitos de espaço de Banach de funções $g$-convexo e espaço de Köthe. Também apresentamos algumas propriedades destes espaços.

No Capítulo 7 relacionamos as teorias de interpolação e somas torcidas de espaço de Köthe.

Nós consideramos as somas torcidas $d_{\Omega_{0}} X_{\theta}$ de $X_{\theta}$, onde $\Omega_{0}: X_{\theta} \rightarrow L_{0}(\mu)$ verifica $(i)$, (ii) e é obtida por diferenciação complexa, i.e., dado $C>1$ fixo, escolhemos $F \in \mathscr{F}$ tal que $\|F\|_{\mathscr{F}}<$ $C\|x\|_{X_{\theta}}$ e $x=F(\theta)$, pondo assim $\Omega_{0}(x)=F^{\prime}(\theta)$. A função $\Omega_{0}$ tem propriedades interessantes como:

- Se $\bar{\Omega}_{0}$ é uma função obtida por diferenciação complexa definida por outra escolha de $F$, então as somas torcidas $d_{\Omega_{0}} X_{\theta}$ e $d_{\bar{\Omega}_{0}} X_{\theta}$ são equivalentes. Além disso, podemos dar uma fórmula explícita para $\Omega_{0}$, i.e.,

$$
\Omega(x)=x\left(\log x_{1}-\log x_{0}\right),
$$

onde $|x|=x_{0}^{1-\theta} x_{1}^{\theta} \mathrm{e}\|x\|_{X_{\theta}}=\left\|x_{0}\right\|_{X_{0}}=\left\|x_{1}\right\|_{X_{1}}$. 
- A soma torcida $d_{\Omega_{0}} X_{\theta}$ pode ser munida de uma norma equivalente a $\|\cdot\|_{d_{\Omega_{0}} X_{\theta}}$.

Neste capítulo vemos que a função $\Omega_{0}$ satisfaz:

(iii) Existe uma constante $\rho\left(\Omega_{0}\right)>0$ tal que

$$
\left\|\Omega_{0}(u x)-u \Omega_{0}(x)\right\|_{X_{\theta}} \leq \rho\left(\Omega_{0}\right)\|u\|_{\infty}\|x\|_{X_{\theta}} .
$$

para todo $u \in L_{\infty}(\mu)$ e todo $x \in X_{\theta}$.

O estudo de auto-extensões de um espaço de Köthe $X$, nos conduz a estudar funções $\Omega: X \rightarrow$ $L_{0}(\mu)$ que verificam as condições $(i)$ e (iii). Estas funções são chamadas de Centralizadores em $X$, as quais também verificam (ii). Esta situação nos leva à seguinte questão :

Dado um centralizador $\Omega$ em $X$, encontrar espaços de Köthe $X_{0}, X_{1}$ tais que $X=\left[X_{0}, X_{1}\right]_{\theta} e$ as somas torcidas $d_{\Omega_{0}} X_{\theta}, d_{\Omega} X$ sejam equivalentes.

Portanto, o estudo das auto-extensões de um espaço de Köthe é reduzido a um problema de extrapolação. Para abordar este problema, nós estudamos um funcional semilinear convexo e $K$-contínuo definido por Gillespie em [25], que chamamos de Indicador de um espaço de Köthe (ver Capítulo 6). Este foi definido por

$$
\Phi_{X}(f)=\sup _{x \in B_{X}} \int_{S} f \log |x| d \mu,
$$

onde $X$ é um espaço de Köthe e $f$ está em um semi-ideal $\mathscr{T}_{X}$ de $L_{1}^{+}=\left\{f \in L_{1}: f \geq 0\right.$ q.s. $\}$. Este funcional está associado a um único espaço de Köthe, i.e., se $\Phi_{X}=\Phi_{Y}$, então $X=Y$. Também provaremos algumas propriedades deste funcional, por exemplo:

- $\operatorname{Se}\left[X_{0}, X_{1}\right]_{\theta}=X_{\theta}=X_{0}^{1-\theta} X_{1}^{\theta}$, então

$$
\Phi_{X_{\theta}}=(1-\theta) \Phi_{X_{0}}+\theta \Phi_{X_{1}}
$$

- para todo funcional semilinear $\Phi$ convexo em um semi-ideal de $L_{1}$, tal que $\Phi_{L_{1}}-\Phi$ seja convexo, existe um espaço de Köthe $X$ tal que $\Phi=\Phi_{X}$.

A importância que desempenha o Indicador de um espaço de Köthe na teoria de extrapolação é que permite reduzir um problema de extrapolação a "solucionar uma equação ". Por exemplo, para demonstrar o resultado de Pisier

Teorema 1.1 (Pisier 1979) [16, pag.498] Se X é um espaço de Köthe p-convexo e q-côncavo, onde $\frac{1}{p}+\frac{1}{q}=1$, então para $\frac{\theta}{2}=\frac{1}{q}, 0<\theta<1$ existe um espaço de Köthe $Y$ tal que

$$
X=L_{2}^{\theta} Y^{1-\theta}
$$


basta encontrar um funcional semilinear convexo $\Phi, \operatorname{com} \Phi_{L_{1}}-\Phi$ convexo, tal que

$$
\Phi_{X}=\frac{\theta}{2} \Phi_{L_{1}}+(1-\theta) \Phi .
$$

Finalmente, no Capítulo 8, além do teorema acima, apresentamos um resultado de extrapolação provado pelo Kalton em 1992. 


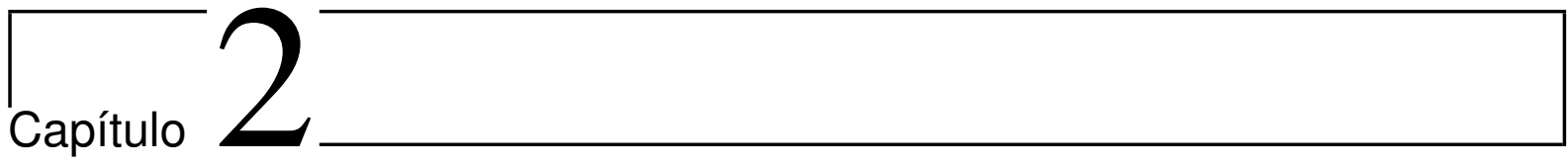

\section{Resultados preliminares}

Apresentamos neste capítulo alguns resultados omitindo suas provas, as quais podem ser encontradas nas referências citadas, pois o objetivo deste capítulo de nosso trabalho é fixar notações e terminologias.

\subsection{Teoria da medida}

Os resultados apresentados nesta seção podem ser consultados em qualquer livro de teoria da medida, ver por exemplo [5] e [28].

\subsubsection{Integração}

Definição 2.1 ( $\sigma$-álgebra) Seja $S$ um conjunto. Uma coleção $\Omega$ de subconjuntos de $S$,é uma $\sigma$-álgebra sobre $S$ se

- $S \in \Omega$,

- Se $A \in \Omega$, então $A^{c} \in \Omega$,

- Se $A_{1}, A_{2}, \ldots \in \Omega$, então $\bigcup_{i=1}^{\infty} A_{i} \in \Omega e \bigcap_{i=1}^{\infty} A_{i} \in \Omega$.

A dupla $(S, \Omega)$ é chamada de espaço mensurável. Todo subconjunto de $S$ que pertence a $\Omega$ é dito mensurável.

A proposição seguinte dá um jeito de construção de $\sigma$-álgebra.

Proposição 2.2 Seja $S$ um conjunto e $\mathscr{F}$ uma coleção de subconjuntos de S. Então existe a menor $\sigma$-álgebra sobre $S$ que contém $\mathscr{F}$. Esta $\sigma$-álgebra é chamada de $\sigma$-álgebra gerada por $\mathscr{F}$. 


\subsection{Teoria da medida}

A Proposição 2.2 nos conduz a definir dois tipos de $\sigma$-álgebra geradas:

Definição 2.3 ( $\sigma$-álgebra gerada em forma enumerável) Seja $\Omega$ uma $\sigma$-álgebra gerada por uma coleção enumerável de conjuntos $\mathscr{F}$. Dizemos que $\Omega$ é gerada em forma enumerável.

Definição 2.4 ( $\sigma$-álgebra de Borel) Seja $S$ um espaço topológico. A $\sigma$-álgebra gerada pelos conjuntos abertos de $S$ é chamada de $\sigma$-álgebra de Borel.

Definição 2.5 (Função mensurável) Sejam $(S, \Omega) e\left(T, \Omega_{0}\right)$ espaços mensuráveis. A função $f:(S, \Omega) \longrightarrow\left(T, \Omega_{0}\right)$ é dita mensurável, se $f^{-1}(A) \in \Omega$ para todo $A \in \Omega_{0}$. Um caso particular desta definição é quando $\Omega_{0}$ é um $\sigma$-álgebra de Borel. Neste caso $f:(S, \Omega) \longrightarrow\left(T, \Omega_{0}\right)$ é dita Borel mensurável.

Quando $T=\mathbb{C}$ na Definição 2.5 temos que:

Proposição 2.6 Se $f, g:(S, \Omega) \longrightarrow \mathbb{C} e \lambda \in \mathbb{C}$, então $\lambda f,|f|, f+g, f g, \max (|f|,|g|) e \frac{f}{g}$ (sempre que o quociente esteja bem definido) são funções Borel mensuráveis.

Definição 2.7 ( $\sigma$-álgebra gerada por funções ) Seja $\left\{\left(S_{i}, \Omega_{i}\right)\right\}_{i \in I}$ uma classe indexada de espaços mensuráveis e seja $f_{i}:(S, \Omega) \rightarrow\left(S_{i}, \Omega_{i}\right)$ uma coleção de funções mensuráveis. A $\sigma$-álgebra gerada pelo conjunto

$$
\left\{f_{i}^{-1}(B): i \in I, B \in \Omega_{i}\right\}
$$

é chamada de $\sigma$-álgebra gerada pelas funções $\left(f_{i}\right)_{i \in I}$.

Definição 2.8 (Função medida) Seja $S$ um conjunto e $\Omega$ uma $\sigma$-álgebra sobre $S$. Uma função medida em $S$ ou simplesmente uma medida em $S$, é uma função $\mu: \Omega \longrightarrow[0,+\infty]$ tal que

- $\mu(\emptyset)=0$,

- $\mu\left(\bigcup_{i=1}^{\infty} A_{i}\right)=\sum_{i=1}^{\infty} \mu\left(A_{i}\right)$ para qualquer coleção enumerável de conjuntos de $S$ disjuntos dois a dois,

A tripla $(S, \Omega, \mu)$ é chamada de espaço de medida.

Definição 2.9 Seja $(S, \Omega, \mu)$ um espaço de medida. A medida $\mu$ é dita $\sigma$-finita se existe uma sequência $A_{1}, A_{2}, \ldots \in \Omega \operatorname{com} \mu\left(A_{i}\right)<\infty$ e $A_{i} \subseteq A_{i+1}$ tal que $S=\bigcup_{i=1}^{\infty} A_{i}$. 


\subsection{Teoria da medida}

Definição 2.10 (Função simples) Seja $(S, \Omega)$ um espaço mensurável e $\mu$ uma medida sobre $(S, \Omega)$. Uma função $f:(S, \Omega) \longrightarrow \mathbb{R}$ é chamada simples não negativa em $S$ se existem conjuntos disjuntos dois a dois $A_{i} \in \Omega$ com $S=\bigcup_{i=1}^{n} A_{i}$ e números reais $a_{i} \geq 0$, para $i=0, \ldots, n$ tais que

$$
f=\sum_{i=1}^{n} a_{i} \cdot 1_{A_{i}},
$$

onde $1_{A_{i}}$ é a função característica do conjunto $A_{i}$. Definimos a integral em $S$ de uma função simples não negativa $f$ em relação a $\mu$ como

$$
\int_{S} f d \mu=\sum_{i=1}^{n} a_{i} \mu\left(A_{i}\right) .
$$

Na definição acima pode ser provado que a integral não depende $\operatorname{dos} A_{i}$ e $a_{i}$.

Definição 2.11 Vamos definir a integral de uma função $f:(S, \Omega) \longrightarrow \mathbb{C}$ mensurável.

- Primeiro consideremos o caso $f:(S, \Omega) \longrightarrow[0,+\infty]$ mensurável. Definimos a integral de f em $S$ como

$$
\int_{S} f d \mu=\sup \left\{\int_{S} g d \mu: g \text { simples não negativa e } g \leq f\right\} .
$$

- Consideremos o caso $f:(S, \Omega) \longrightarrow[-\infty,+\infty]$ mensurável. Seja $f^{+}=\max (f, 0) e$ $f^{-}=\max (-f, 0)$. Então definimos a integral de $f$ em $S$ como

$$
\int_{S} f d \mu=\int_{S} f^{+} d \mu-\int_{S} f^{-} d \mu
$$

- Seja $f:(S, \Omega) \longrightarrow \mathbb{C}$ mensurável. Note que $f=f_{1}+i f_{2}$. Dizemos que $f$ é integrável em $S$, se $f_{1}, f_{2}$ são integráveis em $S$. Definimos

$$
\int_{S} f d \mu=\int_{S} f_{1} d \mu+i \int_{S} f_{2} d \mu
$$

- Seja $A \in \Omega$. Dizemos que $f:(S, \Omega) \longrightarrow \mathbb{C}$ é integrável em $A$, se $f \cdot 1_{A}$ é integrável em $S$. Sua integral está dada por

$$
\int_{A} f d \mu=\int_{S} f \cdot 1_{A} d \mu
$$




\subsection{Teoria da medida}

Definição 2.12 (Espaço $L_{p}$ ) Seja $(S, \Omega, \mu)$ um espaço mensurável. Para $1 \leq p<\infty$, definimos o espaço normado

$$
L_{p}(S, \mu)=\left\{f: S \longrightarrow \mathbb{C} \text { mensurável }: \int_{S}|f|^{p} d \mu<\infty\right\},
$$

com norma $\|f\|_{L_{p}}=\left(\int_{S}|f|^{p} d \mu\right)^{1 / p}$.

O espaço $L_{p}(S, \mu)$ é um espaço de Banach. Também denotamos $L_{p}(S, \mu)$ simplesmente por $L_{p}(\mu)$ ou $L_{p}$.

\subsubsection{Tipos de convergências}

Vamos definir alguns tipos de convergência de funções que aparecem no trabalho.

Definição 2.13 (Convergências de funções) Seja $(S, \Omega, \mu)$ um espaço de medida e $f_{n}: S \longrightarrow$ $\mathbb{C}$ uma sequência de funções mensuráveis.

- Convergência quase-sempre. Diz-se que a sequência de funções $f_{n}: S \longrightarrow \mathbb{C}$ converge quase-sempre em $S$ (converge q.s.) para uma função mensurável $f: S \longrightarrow \mathbb{C}$, se existe um conjunto mensurável A satisfazendo $\mu(A)=0$, tal que $\left(f_{n}\right)_{n}$ converge pontualmente para $f$ em $S / A$.

Denotamos esta convergência por $f_{n} \longrightarrow f$ q.s. ou $f_{n} \stackrel{\text { q.s. }}{\longrightarrow} f$

- Convergência em medida. Diz-se que a sequência de funções $f_{n}: S \longrightarrow \mathbb{C}$ converge em medida para uma função mensurável $f: S \longrightarrow \mathbb{C}$, se para todo $\varepsilon>0$ e todo $A$ mensurável, $\operatorname{com} \mu(A)<\infty$, temos

$$
\lim _{n \rightarrow \infty} \mu\left(\left\{s \in A:\left|f(s)-f_{n}(s)\right| \geq \varepsilon\right\}\right)=0 .
$$

Proposição 2.14 [5, pág. 173, 180] Seja $f_{n}:(S, \Omega, \mu) \longrightarrow \mathbb{C}$ uma sequência de funções em um espaço de medida.

- Se $\mu$ é uma medida $\sigma$-finita e $\left(f_{n}\right)_{n}$ converge em medida para uma função $f$, então existe uma subsequência de $\left(f_{n}\right)_{n}$ que converge q.s. para $f$.

- Se $\left(f_{n}\right)_{n}$ converge q.s. para uma função $f$, então $\left(f_{n}\right)_{n}$ converge em medida para $f$.

Definição 2.15 (Espaço $\left.L_{0}\right)$ Seja $(S, \Omega, \mu)$ um espaço mensurável. Definimos o espaço vetorial

$$
L_{0}(S, d \mu)=\{f: S \longrightarrow \mathbb{C} \text { função }: \text { fé mensurável }\}
$$

munido da topologia da convergência em medida. 


\subsection{Teoria da medida}

Também denotamos $L_{0}(S, d \mu)$ simplesmente por $L_{0}(\mu)$ ou $L_{0}$.

Proposição 2.16 [5, pág. 178]Seja $(S, \Omega, \mu)$ um espaço mensurável. Se $1 \leq p<\infty$, então $L_{p}(S, \mu)$ é mergulhado em $L_{0}(S, \mu)$.

Teorema 2.17 [28, pág. 67] Seja $\left(f_{n}\right)_{n}$ uma sequência em $L_{p}$ tal que $\left\|f_{n}-f\right\|_{L_{p}} \rightarrow 0$, se $n \rightarrow \infty$. Então existe uma subsequência de $\left(f_{n}\right)_{n}$ que converge q.s. para $f$.

Teorema 2.18 [4, pág. 102] O espaço $L_{p}(S, \Omega, \mu)$ é separável, se $\mu$ é uma medida $\sigma$-finita e $\Omega$ é gerada em forma enumerável.

\subsubsection{Integral de Bochner}

Nesta subseção nós apresentamos alguns resultado da teoria de integração em espaços normados que podem ser encontrados na referência [7].

Definição 2.19 (Função simples) Sejam $(S, \Omega, \mu)$ um espaço de medida e $X$ um espaço normado. Uma função $f: S \longrightarrow X$ é chamada de simples, se existem $x_{1}, \ldots, x_{n} \in X e$ $S_{1}, \ldots, S_{n} \in \Omega$ tais que $f=\sum_{k=1}^{n} x_{k} \cdot 1_{S_{k}}$. A integral de uma função simples em $S$ é definida por

$$
\int_{S} f d \mu=\sum_{k=1}^{n} x_{k} \mu\left(S_{k}\right) .
$$

Definição 2.20 (Função fortemente mensurável) Uma função $f: S \longrightarrow X$ é fortemente mensurável ou $\mu$-mensurável, se existe uma sequência de funções simples $f_{n}$ tais que

$$
\lim _{n \rightarrow \infty}\left\|f(s)-f_{n}(s)\right\|_{X}=0 \text { q.s. }
$$

Definição 2.21 (Função fracamente mensurável) Uma função $f: S \longrightarrow X$ é fracamente mensurável ou fraca $\mu$-mensurável, se para todo $\varphi \in X^{\prime}$ a função $\varphi \circ f$ é fortemente mensurável.

Definição 2.22 (Função essencialmente separável) Uma função $f: S \longrightarrow X$ é essencialmente separável, se existe $A \subset S$, com $\mu(A)=0$ e tal que $f(S \backslash A)$ é um subconjunto separável (em norma) de X.

Teorema 2.23 (Teorema de Pettis) [7, pág. 42] Uma função $f: S \longrightarrow X$ é fortemente mensurável se, e somente se, $f$ é fracamente mensurável e essencialmente separável.

Observação 2.24 Quando X é um espaço separável, a definiçao de fracamente mensurável e fortemente mensurável são equivalentes. 


\subsection{Funções diferenciáveis}

Definição 2.25 (Função Bochner integrável) Uma função $f: S \longrightarrow X$ é chamada de Bochner integrável em $S$, se existe uma sequência de funções simples $\left(f_{n}\right)_{n}$ tal que

$$
\lim _{n \rightarrow \infty} \int_{S}\left\|f_{n}(s)-f(s)\right\|_{X} d \mu=0 .
$$

A integral de Brochner de f em $S$ é definida por

$$
\int_{S} f d \mu=\lim _{n \rightarrow \infty} \int_{S} f_{n} d \mu .
$$

A integral de Brochner de $f$ em $S$ não depende da escolha da sequência de funções simples $\left(f_{n}\right)_{n}$.

Teorema 2.26 Seja $f: S \longrightarrow X$ uma função fortemente mensurável. A função $f$ é Bochner integrável em $S$ se, e somente se,

$$
\int_{S}\|f(s)\|_{X} d \mu<\infty
$$

\subsection{Funções diferenciáveis}

\subsubsection{Funções analíticas}

Para o estudo desta seção pode-se ver [15].

Definição 2.27 (Funcão holomorfa) Seja $X$ um quase-espaço de Banach e $U \subseteq \mathbb{C}$ aberto. Dizemos que uma função $f: U \longrightarrow X$ é holomorfa em $z_{0} \in U$, se existe uma sequência $\left(a_{n}\right)_{n}$ de $X$ e $U_{0}$ subconjunto aberto de $U$ tais que

$$
f(z)=\sum_{i=1}^{\infty} a_{n}\left(z-z_{0}\right)^{n}
$$

para todo $z \in U_{0}$.

Proposição 2.28 Seja $X$ um quase-espaço de Banach, $U \subseteq \mathbb{C}$ aberto e $f_{n}: U \longrightarrow X$ uma sequência de funções holomorfas. Se $f_{n}$ converge uniformemente em todo subconjunto compacto de U para uma função $f$, então $f$ é holomorfa em $U$.

Proposição 2.29 Seja $f: U \subseteq \mathbb{C} \longrightarrow \mathbb{C}$ uma função holomorfa, onde $U$ é aberto e conexo. Se $f$ é zero em uma sequência de pontos distintos de $U$ que tem ponto límite em $U$, então $f$ é zero em $U$.

Teorema 2.30 (Teorema da aplicação de Riemann) [23, pág. 306] Seja A um subconjunto proprio de $\mathbb{C}$ simplesmente conexo e $\mathbb{D}$ o disco unitário. Se $z_{0} \in A$, então existe uma única aplicação conforme $\varphi: A \longrightarrow \mathbb{D}$ tal que $\varphi\left(z_{0}\right)=0$ e $\varphi^{\prime}\left(z_{0}\right)>0$. 


\subsubsection{Funções de Lipschitz}

Definição 2.31 (Funcão de Lipschitz) Sejam X e Y espaços de Banach. A função $f: X \longrightarrow Y$ é uma função de Lipschitz, se existe uma constante $K>0$ tal que

$$
\left\|f\left(x_{0}\right)-f\left(x_{1}\right)\right\|_{Y} \leq K\left\|x_{0}-x_{1}\right\|_{Y}
$$

para todo $x_{0}, x_{1} \in X$.

Teorema 2.32 [27, pág. 19] Seja X um espaço de Banach reflexivo. Então toda função de Lipschitz $f: \mathbb{R} \longrightarrow X$ é quase-sempre diferenciável.

\subsection{Espaços de Banach reticulados}

Para o estudo desta seção, ver a referência [11, cap. 1].

Definição 2.33 (Espaços de Banach reticulados) Um espaço de Banach X sobre $\mathbb{R}$, com uma ordem parcial $\leq$,é chamado de espaço de Banach reticulado, se

- $x \leq y$, implica que $x+z \leq y+z$ para todo $x, y, z \in X$,

- $a \cdot x \geq 0$, para todo $x \geq 0$ e todo real $a \geq 0$,

- para todo $x, y \in X$, existem o supremo e o infimo de $\{x, y\}$, que denotamos respectivamente por $x \vee y$ e $x \wedge y$,

- se $|x| \leq|y|$, então $\|x\|_{X} \leq\|y\|_{X}$, onde $|x|=x \vee(-x)$.

Denotamos por $X^{+}=\{x \in X: x \geq 0\}$.

Definição 2.34 (Elementos disjuntos) Seja X um espaço de Banach reticulado. Os elementos $x, y \in X$ são disjuntos, se $|x| \wedge|y|=0$.

Definição 2.35 (Cone) Seja $X$ um espaço de Banach reticulado. Um Cone de X é um subconjunto $C$ de $X$ tal que para todo $x, y \in C$ e $\lambda \geq 0$ temos que $\lambda \cdot x \in C$ e $x+y \in C$.

Definição 2.36 (Cone $L_{1}^{+}(\mu)$ ) Definimos um cone de $L_{1}(\mu)$ por

$$
L_{1}^{+}(\mu)=\left\{f \in L_{1}^{+}(\mu): f \geq 0 \text { q.s. }\right\} .
$$

Simplesmente denotamos $L_{1}^{+}(\mu)$ por $L_{1}^{+}$. 


\subsection{Espaços de Banach reticulados}

Definição 2.37 (Espaço de Banach de função) Seja $(S, \mu)$ um espaço de medida. Um espaço de Banach cujos elementos são funções $\mu$-mensuráveis de valores reais ou complexos, e tal que

$$
|f| \leq|g| \Rightarrow\|f\|_{X} \leq\|g\|_{X}
$$

é chamado de espaço de Banach de funções .

Observe que um espaço de Banach de funções é um espaço de Banach reticulado.

Definição 2.38 (Espaços $p$-convexos e $q$-côncavos) Seja $X$ um espaço de Banach de funções com valores em $\mathbb{C} e 1 \leq p<\infty$. O espaço $X$ é dito $p$-convexo se

$$
\left\|\left(\sum_{i=1}^{n}\left|x_{i}\right|^{p}\right)^{1 / p}\right\|_{X} \leq C_{0}\left(\sum_{i=1}^{n}\left\|x_{i}\right\|_{X}^{p}\right)^{1 / p}
$$

para todo $n \in \mathbb{N}$ e alguma constante $C_{0}>0$.

O espaço X é dito q-côncavo, com $1 \leq q<\infty$, se

$$
C_{1}\left\|\left(\sum_{i=1}^{n}\left|x_{i}\right|^{q}\right)^{1 / q}\right\|_{X} \geq\left(\sum_{i=1}^{n}\left\|x_{i}\right\|_{X}^{q}\right)^{1 / q}
$$

para todo $n \in \mathbb{N}$ e alguma constante $C_{1}>0$.

Neste trabalho, vamos assumir que $C_{0}=C_{1}=1$.

Proposição 2.39 Sejam $1 \leq p<\infty e 1 \leq q \leq \infty$, tais que $\frac{1}{p}+\frac{1}{q}=1$. Se X é um espaço p-convexo (respectivamente q-côncavo), então o espaço dual $X^{\prime}$ é um espaço q-côncavo (respectivamente q-convexo).

Proposição 2.40 Seja X e Y espaços de Banach de funções e assuma que $Y$ é isomorfo (em sentido topológico) a um subespaço de X. Então

- Se X é p-convexo e q-côncavo para algum $1<p \leq 2$ e $q<\infty$, então Y é p-convexo.

- Se X é q-côncavo para algum $q \geq 2$, então $Y$ é q-côncavo.

\subsection{1 $p$-potências de espaços de funções}

As definições e a prova dos resultados desta seção podem ser consultados em [24, Pág. 55].

Definição 2.41 Dado $0<p<\infty$ e X um espaço de Banach de função, definimos a p-potências de X por

$$
X^{(p)}=\left\{x \text { mensurável: }|x|^{1 / p} \in X\right\} .
$$


Observação 2.42 O conjunto $X^{(p)}$ é um espaço vetorial.

Proposição 2.43 Seja X um espaço de Banach de funções . Definimos uma função em $X^{(p)}$ por

$$
\|x\|_{X^{(p)}}=\left\||x|^{1 / p}\right\|_{X}^{p}
$$

Se $0<p \leq 1$, então $\left(X^{(p)},\|\cdot\|_{X^{(p)}}\right)$ é um espaço de Banach funções.

\subsubsection{Normas de ordem contínua}

Para consultar a prova dos resultados ver a referência [20, pág. 86]

Definição 2.44 Seja X um espaços de Banach reticulado. A norma $\|\cdot\|_{X}$ é chamada de norma de ordem contínua, se para toda rede decrescente $\left(x_{\alpha}\right)_{\alpha}$ tal que $\inf \left\{x_{\alpha}\right\}=0$, temos que inf $\left\|x_{\alpha}\right\|_{X}=$ 0 .

Proposição 2.45 Seja X um espaços de Banach reticulado. As seguintes afirmações são equivalentes

1. $\|\cdot\|_{X}$ é uma norma de ordem contínua.

2. Toda sequência monótona limitada é convergente.

3. Toda sequência disjunta limitada de $X^{+}$converge em norma para zero.

Exemplo 2.46 O espaço $L_{p}(\mu)$ munido da norma usual, onde $1 \leq p<\infty$, tem norma de ordem contínua.

\subsubsection{Espaço de Banach de sequências (E.B.S)}

As definições desta seção podem ser encontradas [29, pág. 1161].

Um espaço de Banach de sequências (E.B.S) X, é um espaço de sequências normado e completo tal que

- Os vetores da base canônica $e_{n}$ pertencem a $X$.

- Se $\left|y_{n}\right| \leq\left|x_{n}\right|$, onde $x \in X$, então $y \in X$ e $\|y\|_{X} \leq\|x\|_{X}$.

- Para todo $n \in \mathbb{N}$, o funcional $x \longmapsto x_{n}$ é contínuo. 


\subsection{Espaços quase-normados}

- A bola unitária $B_{X}$ é fechada na topologia da convergência pontual.

Definimos o espaço dual de Köthe ou dual de Köthe de um E.B.S, que denotamos por $X^{*}$, como

$$
X^{*}=\left\{x^{*}: \sum_{n=1}^{\infty}\left|x_{n} \| x_{n}^{*}\right|<\infty, \text { para todo } x \in B_{X}\right\}
$$

munido da norma

$$
\|x\|_{X^{*}}=\sup _{x \in B_{X}} \sum_{n=1}^{\infty}\left|x_{n} \| x_{n}^{*}\right| .
$$

\subsection{Espaços quase-normados}

Definição 2.47 Seja $X$ um espaço vetorial. Uma função $\|\cdot\|_{X}: X \longrightarrow[0, \infty)$ é chamada quase-norma em $X$, se verifica:

- se $\|x\|_{X}=0$, então $x=0$,

- $\|\lambda x\|_{X}=|\lambda| \cdot\|x\|_{X}$ para todo $\lambda$ escalar e $x \in X$,

- existe um $C>0$ tal que para todo $x, y \in X$, temos

$$
\|x+y\|_{X} \leq C \cdot\left(\|x\|_{X}+\|y\|_{X}\right) .
$$

$O$ par $\left(X,\|\cdot\|_{X}\right)$ é chamado de espaço quase-normado. A menor constante que verifica a desigualdade acima é chamada módulo de concavidade da quase-norma.

Definição 2.48 (Quase-normas equivalentes) Seja X um espaço quase-normado e $\|\cdot\|,\||\cdot|\|$ duas quase-normas em $X$. Dizemos que $\|\cdot\| e\||\cdot|\|$ são quase-normas equivalentes se existem constantes positivas $M$ e $m$ tais que

$$
m \cdot\|x\| \leq\|\| x\|\leq M \cdot\| x \|
$$

para todo $x \in X$.

Sejam $r>0$ e $x_{0} \in X$. Os conjuntos da forma $B_{r}\left(x_{0}\right)=\left\{x \in X:\left\|x-x_{0}\right\|_{X}<r\right\}$ induzem uma topologia em $X$, que chamamos de topologia induzida por $\|\cdot\|_{X}$. Note que uma quase-norma nem sempre é uma métrica (pode não verifica a desigualdade triangular). Então para definir uma noção de completude em um espaço quase-normado, precisamos de uma métrica que induza a mesma topologia que induz $\|\cdot\|_{X}$. O seguinte teorema soluciona este problema: 


\subsection{Espaços quase-normados}

Teorema 2.49 (Teorema de Aoki-Rolewicz) [2] Se X é um espaço quase-normado cuja quase-norma satisfaz

$$
\|x+y\|_{X} \leq C \cdot\left(\|x\|_{X}+\|y\|_{X}\right)
$$

para algum $C>1$ e para todo $x, y \in X$, então $\|\cdot\|$ dada por

$$
\||x|\|=\inf \left\{\left(\sum_{k=1}^{n}\left\|x_{k}\right\|_{X}^{p}\right)^{1 / p}: x=\sum_{k=1}^{n} x_{k}\right\}
$$

para todo $x \in X$, é uma quase-norma em $X$ que verifica

$$
\|\| x\left\|\left|\leq\|x\|_{X} \leq 2 C \cdot\||x \|| \text { para todo } x \in X\right.\right.
$$

$e$

$$
\left\|\left|x+y\|\|^{p} \leq\|\| x\right|\right\|^{p}+\|\mid y\|^{p}
$$

para todo $x, y \in X$, onde p é o número tal que $C=2^{1 / p-1}$.

Observação 2.50 O Teorema de Aoki-Rolewicz garante que a função d definida por

$$
d(x, y)=\|x-y\| \|^{p} \text { para todo } x, y \in X,
$$

define uma métrica em $X$ que induz a mesma topologia que $\|\cdot\|_{X}$.

Este fato motiva a seguinte definição :

Definição 2.51 (Espaço quase-Banach) Seja X um espaço quase-normado. Dizemos que Xé um espaço quase-Banach, se $(X, d)$ é um espaço métrico completo, onde

$$
d(x, y)=\|\mid x-y\|^{p} \text { para todo } x, y \in X
$$

e ||$\cdot \mid \|$, p são dados pelo Teorema de Aoki-Rolewicz.

Definição 2.52 (Operador linear limitado) Seja $\left(X,\|\cdot\|_{X}\right)$ e $\left(Y,\|\cdot\|_{Y}\right)$ espaços quasenormados. Um operador linear $T: X \longrightarrow Y$ é chamado limitado, se

$$
\|T\|=\sup _{\|x\|_{X} \leq 1}\|T(x)\|_{Y}<\infty .
$$

Definição 2.53 (Isomorfismo entre espaços quase-normados ) Seja $\left(X,\|\cdot\|_{X}\right)$ e $\left(Y,\|\cdot\|_{Y}\right)$ espaços quase-normados. Um operador linear limitado bijetor $T: X \rightarrow Y$ é chamado isomorfismo de $X$ em $Y$, se $T^{-1}$ é um operador linear limitado. Dizemos que dois espaços quase-normados $X$ e $Y$ são isomorfos, se existe um isomorfismo de $X$ em $Y$.

Outras definições, como espaço quociente de espaços quase-normados são definidas de maneira análoga ao caso de espaços normados. Resultados como os teoremas da aplicação aberta e do grafo fechado também são válidos para espaços quase-Banach (Ver [28, Pág. 43-54]). 
Proposição 2.54 (Existência de função de escolha homogênea limitada) Seja $T: X \longrightarrow Y$ um operador linear sobrejetor entre espacos quase-Banach complexos. Então existe uma função $\phi: Y \longrightarrow X$ e uma constante $C>0$ tal que:

1. $\|\phi(y)\|_{X} \leq C\|y\|_{Y}$ para todo $y \in Y$,

2. $T(\phi(y))=y$ para todo $y \in Y$,

3. $\phi(\lambda \cdot y)=\lambda \cdot \phi(y)$ para todo $y \in Y$ e $\lambda$ complexo.

Demonstração. Dado que $T$ é sobrejetora, pelo teorema da aplicação aberta temos que existe uma constante $C>0$ tal que $\frac{1}{C} B_{Y} \subseteq T\left(B_{X}\right)$. Vamos definir inicialmente uma função $\phi$ em $S_{Y}=\left\{y \in Y:\|Y\|_{Y}=1\right\}$. Considere sobre $S_{Y}$ a relação de equivalência $y_{1} \sim y_{1}$ se, e somente se, existe $\lambda$ complexo com $|\lambda|=1$ tal que $y_{2}=\lambda y_{1}$. Escrevemos como $\bar{y}$ a classe de equivalência do elemento $y$. $\mathrm{O}$ axioma da escolha garante a existência de um conjunto $J=\cup_{\alpha \in I}\left\{y_{\alpha}\right\}$ que contém um único elemento de cada classe de equivalência. Seja $A_{\alpha}=\left\{x \in B_{X}: y_{\alpha}=T\left(C x_{\alpha}\right)\right\}$. Note que $\left(A_{\alpha}\right)_{\alpha \in I}$ é uma coleção de conjuntos não vazios disjuntos dois a dois, portanto, pelo axioma da escolha, existe um conjunto $A=\cup_{\alpha \in I}\left\{x_{\alpha}\right\}$ que contém um único elemento de $A_{\alpha}$, isto é, $A \cap A_{\alpha}=\left\{x_{\alpha}\right\}$. Para cada $y_{\alpha} \in J$, definamos $\phi\left(y_{\alpha}\right)=C x_{\alpha}$. Deste modo $\left\|\phi\left(y_{\alpha}\right)\right\|_{X} \leq C$ para todo $\alpha \in I$. Agora dado $y \in S_{Y}$, definamos $\phi(y)=y_{\alpha}$ se $y \in \overline{y_{\alpha}}$. Também, para cada $\lambda$ escalar e $y \in S_{Y}$ definamos $\phi(\lambda y)=\lambda \phi(y)$. Logo, para $y \in Y$ não nulo, seja

$$
\phi(y)=\|y\|_{Y} \phi\left(\frac{y}{\|y\|_{Y}}\right) .
$$

É imediato ver que $\phi$ satisfaz as condições 1,2 e 3 .

\subsection{Outros resultados}

Teorema 2.55 (Desigualdade de Jensen) [28, pág. 61] Seja $(S, \Omega, \mu)$ um espaço de medida tal que $\mu(S)<\infty$. Seja $\varphi: I \subseteq \mathbb{R} \rightarrow \mathbb{R}$ uma função convexa, onde I é um intervalo. Se $g: S \rightarrow I$ é uma função integrável, então

$$
\varphi\left(\frac{1}{\mu(S)} \cdot \int_{S} g d \mu\right) \leq \frac{1}{\mu(S)} \cdot \int_{S}(\varphi \circ g) d \mu .
$$

O próximo corolário é uma consequência da desigualdade de Jensen

Corolário 2.56 (Versão da desigualdade de Jensen para funções de densidades) Seja $(S, \Omega, \mu)$ onde $S$ é um subconjunto da reta real. Seja $f: \mathbb{R} \longrightarrow \mathbb{R}$ uma função não negativa tal que

$$
\int_{-\infty}^{\infty} f(t) d t=1
$$




\subsection{Outros resultados}

Seja $g$ uma função de valor real mensurável e $\varphi: \operatorname{Im}(\mathrm{g}) \rightarrow \mathbb{R}$ uma função convexa. Então

$$
\varphi\left(\int_{-\infty}^{\infty} g(t) f(t) d t\right) \leq \int_{-\infty}^{\infty}(\varphi \circ g)(t) f(t) d t .
$$

Teorema 2.57 (Teorema de Komlós) [10] Seja $\mu$ uma medida $\sigma$-finita. Se $\left(f_{n}\right)_{n}$ é uma sequência em $L_{1}(\mu)$ limitada, então existe uma subsequência $\left(g_{n}\right)_{n}$ de $\left(f_{n}\right)_{n}$ e uma função $g \in L_{1}(\mu)$ tal que toda subsequência $\left(h_{n}\right)_{n} d e\left(g_{n}\right)_{n}$ é Cesàro-convergente para $g$, isto é,

$$
\frac{1}{n} \sum_{k=1}^{n} h_{k} \stackrel{q . s .}{\rightarrow} g .
$$

Teorema 2.58 (Teorema de Convergência Monótona) [28, pág. 21] Seja $\left(f_{n}\right)_{n}$ uma sequência crescente de funções em $L_{1}(S, \mu)$ tal que

$$
\sup _{n} \int f_{n} d \mu<\infty
$$

Se $\left(f_{n}\right)_{n}$ converge q.s. em $S$ para uma função $f$, então $f \in L_{1}(S, \mu) e\left\|f_{n}-f\right\|_{L_{1}} \longrightarrow 0$, se $n \rightarrow \infty$.

Teorema 2.59 (Teorema de Convergência Dominada) [28, pág. 26] Seja $\left(f_{n}\right)_{n}$ uma sequência de funções mensuráveis em $S$ de valores complexos tais que $f_{n} \rightarrow f$ q.s. Se existe uma função $g \in L_{1}(\mu)$ tal que $\left|f_{n}(s)\right| \leq g_{n}(s)$ para todo $s \in S$, então $f \in L_{1}(\mu)$,

$$
\lim _{n \rightarrow \infty}\left\|f_{n}-f\right\|_{L_{1}}=0
$$

$e$

$$
\lim _{n \rightarrow \infty} \int_{S} f_{n} d \mu=\int_{S} f d \mu .
$$

Teorema 2.60 (Desigualdade de Hölder) [28, pág. 62] Se $f \in L_{p}$ e $g \in L_{q} \operatorname{com} 1 / p+1 / q=1$, então $f g \in L_{1} e$

$$
\int|f g| d \mu \leq\|f\|_{L_{p}}\|g\|_{L_{q}} \text {. }
$$

Teorema 2.61 (Teorema de Egorov) [4, pág. 81] Seja $(S, \mu)$ um espaço de medida e $E$ um conjunto tal que $\mu(E)<\infty$. Seja $\left(f_{n}\right)_{n}$ uma sequência de funções mensuráveis tal que $f_{n} \rightarrow f$ q.s. em $E$, onde $f$ é uma função mensurável. Então para $\varepsilon>0$, existe um conjunto mensurável $A \subseteq E$ tal que $\mu(E \backslash A)<\varepsilon e\left(f_{n}\right)_{n}$ converge uniformemente para $f$ em $A$. 


\subsection{Outros resultados}

Usando o teorema acima demostramos o seguinte resultado:

Corolário 2.62 Seja $(S, \mu)$ um espaço de medida, onde $\mu$ é $\sigma$-finita. Seja $\left(f_{n}\right)_{n}$ uma sequência de funções mensuráveis tal que $f_{n} \rightarrow f$ q.s. em $S$, onde $f$ é uma função mensurável. Então , existe uma sequência de conjuntos $\left(A_{n}\right)_{n}$ mensuráveis, tais que, $A_{n} \subseteq A_{n+1}, \mu\left(S \backslash \cup_{n} A_{n}\right)=0$ e $\left(f_{n}\right)_{n}$ converge uniformemente para $f$ em cada $A_{n}$.

Teorema 2.63 [5, pág. 180] Seja $\mu$ uma medida $\sigma$-finita. Então a bola unitária $B_{L_{1}(\mu)} \subseteq L_{0}(\mu)$ é fechada na topologia da convergência em medida.

Teorema 2.64 (Teorema de Hahn-Banach para funcionais contínuos) [12, pág. 40] Seja X um espaço normado real (ou complexo) e Y um subespaço normado de X. Se $\varphi$ é um funcional linear contínuo em $Y$, então existe um funcional linear contínuo $\bar{\varphi}$ em $X$, tal que $\bar{\varphi}(y)=\varphi(y)$ para todo $y \in Y$ e $\|\bar{\varphi}\|=\|\varphi\|$.

Teorema 2.65 (Teorema de Hahn-Banach geométrico) [12, pág. 43] Seja X um espaço de Banach real. Sejam A, B subcojuntos convexos e disjuntos de X. Se A é aberto, então existe um funcional linear contínuo $\varphi: X \longrightarrow \mathbb{R}$ tal que

$$
\varphi(b)<\varphi(\text { a) para todo } a \in A \text { e todo } b \in B \text {. }
$$

Teorema 2.66 (Teorema de Separação) [12, pág. 43] Seja $X$ um espaço normado real ou complexo e $C \subset X$ fechado convexo. Se $x_{0} \notin C$, então existe $\varphi \in X^{\prime}$ tal que

$$
\operatorname{Re}\left(\varphi\left(x_{0}\right)\right)>\sup _{x \in C} \operatorname{Re}(\varphi(x)) .
$$

Teorema 2.67 [7, pág. 59] Seja $\mu$ é uma medida $\sigma$-finita e $\varphi \in\left(L_{1}(\mu)\right)^{\prime}$. Então existe $u \in L_{\infty}(\mu)$ único tal que

$$
\varphi(f)=\int u f d \mu .
$$

Proposição 2.68 [6, pág. 97] Seja $T: X \longrightarrow Y$ uma aplicação linear sobrejetora entre espaços vetoriais. Então existe uma aplicação linear $S: Y \longrightarrow X$ tal que $T S=i d_{Y}$.

Proposição 2.69 [9, pág. 3] Sejam $Y, X, Z, R, W$ e $S$ espaços quase-Banach. Suponha que o seguinte diagrama comuta



onde $i_{k}$ é injetor e $j_{k}$ é sobrejetor com $\operatorname{Im}\left(i_{k}\right)=\operatorname{ker}\left(j_{k}\right)$ para $k=1,2$. Se $\alpha, \beta$ são isomorfismos topológicos, então $\gamma$ é um isomorfismo topológico. 


\subsection{Outros resultados}

Teorema 2.70 [21] Sejam X e Y espaços de Banach e $T: X \longrightarrow Y$ um operador linear limitado. As seguintes afirmações são equivalentes:

1. $T(X)$ éfechado.

2. $X / \operatorname{ker}(T)$ é isomorfo a $T(X)$.

3. Existe uma constante $C$ tal que para todo $x \in X$, existe $y \in X \operatorname{com} T(x)=T(y) e$ $\|y\|_{X} \leq C\|T(x)\|_{Y}$.

Teorema 2.71 (Banach-Dieudonné) [12, pág. 125] Seja X um espaço de Banach e $K$ um subconjunto convexo em $X^{\prime}$. Se $K \cap n B_{X}$ é $w^{\star}$-fechado para todo $n \in \mathbb{N}$, então $K$ é $w^{\star}$-fechado.

Teorema 2.72 [12, pág. 70] Seja X um espaço de Banach real. Se K é um subconjuto convexo $w^{\star}$-fechado de $X^{\prime}$ e $\varphi \in X^{\prime} \backslash K$, então existe $x_{0} \in X$ tal que $\varphi\left(x_{0}\right)>\sup _{\Psi \in K} \Psi\left(x_{0}\right)$.

Definição 2.73 (Funcional de Minkowski) [28, pág. 24] Seja X um espaço vetorial sobre um corpo $(\mathbb{R}$ ou $\mathbb{C})$. Seja $K$ um subconjunto de $X$. O funcional de Minkowski do conjunto $K$, $\rho_{K}: X \longmapsto[0, \infty]$, é definido por

$$
\rho_{K}(x)= \begin{cases}\inf \left\{\alpha>0: \alpha^{-1} x \in K\right\}, & \text { se } \alpha^{-1} x \in K, \text { para algum } \alpha>0 \\ \infty, & \text { se } \alpha^{-1} x \notin K, \text { para todo } \alpha>0 .\end{cases}
$$

Definição 2.74 [28, pág. 6,24-25] Seja $X$ um espaço vetorial sobre um corpo ( $\mathbb{R}$ ou $\mathbb{C}$ ). Seja $K$ um subconjunto de $X$.

- Ké chamado de balançado, se para todo $|\lambda| \leq 1$ e $x \in K$, temos que $\lambda x \in K$

- $K$ é chamado de absorvente, se para $x \in X$, existe $\lambda>0$ escalar tal que $\lambda^{-1} x \in K$.

Proposição 2.75 (Propriedades do funcional de Minkowski) [28, pág. 25] Seja $\rho_{K}$ o funcional de Minkowski e suponha que K é absorvente e convexo. Então

- $\rho_{K}(\lambda x)=\lambda \rho_{K}(x)$ para todo $\lambda \geq 0$ escalar e $x \in X$,

- $\rho_{K}(x+y) \leq \rho_{K}(x)+\rho_{K}(y)$, para todo $x, y \in X$,

- $\rho_{K}$ é uma seminorma, se $K$ é balançado. 


\subsection{Outros resultados}

Lema 2.76 [18, pág. 256] Suponha $\varepsilon>0 e$

$$
B_{\varepsilon}=\log \left(\sum_{k=1}^{\infty}\left(\frac{1}{k}\right)^{1+\varepsilon}\right)
$$

Se $\xi_{1} \geq \xi_{2}, \ldots \geq \xi_{n} \geq 0$ e $\sum_{k=1}^{n} \xi_{k}=1$, então

$$
\sum_{k=1}^{n} \xi_{k} \log k \leq \sum_{k=1}^{n} \xi_{k} \log \frac{1}{\xi_{k}} \leq(1+\varepsilon) \sum_{k=1}^{n} \xi_{k} \log k+B_{\varepsilon} .
$$

Proposição 2.77 [3] Se $\left(a_{n}\right)_{n},\left(b_{n}\right)_{n},\left(c_{n}\right)_{n}$ são sequências de números positivos tais que $a_{n}=$ $b_{n} c_{n}$,

$$
\begin{gathered}
\lim _{n \rightarrow \infty} a_{n}=a, \\
\lim _{n \rightarrow \infty}\left(\frac{1}{n}\left(b_{1}+b_{2}+\ldots+b_{n}\right)\right)=b \\
\lim _{n \rightarrow \infty}\left(\frac{1}{n}\left(c_{1}+c_{2}+\ldots+c_{n}\right)\right)=c,
\end{gathered}
$$

$e$

então $a \leq b c$.

Definição 2.78 (Subespaço normante) Seja $X^{\prime}$ o espaço dual usual de X. Um subespaço normado $Y$ de $X^{\prime}$, é chamado de subespaço normante de $X^{\prime}$ se

$$
\|x\|_{X}=\sup _{x^{\prime} \in B_{Y}}\left|x^{\prime}(x)\right| .
$$

Definição 2.79 (Predual) [26] Seja Y um espaço de Banach. Um espaço de Banach $Y_{*}$ é um predual para $Y$, se o dual $Y_{*}^{\prime}$ é isométrico a $Y$.

Teorema 2.80 (Extensão uniformemente contínua de funções ) [14, pág. 176 ] Seja M um subespaço métrico de algum espaço métrico X e Y um espaço métrico completo.

Se $f: M \longrightarrow Y$ é uma função uniformemente contínua, então existe uma única extensão $\bar{f}: \bar{M} \longrightarrow Y$ uniformemente contínua. A extensão está definida por

$$
\bar{f}(x)=\lim _{n \rightarrow \infty} f\left(x_{n}\right),
$$

onde $\left(x_{n}\right)_{n}$ é qualquer sequência de $M$ que converge para $x$.

Teorema 2.81 (Teorema da interseção de Cantor) [22, pág. 134] Seja X um espaço de Banach. Então $X$ é reflexivo se, e somente se, para qualquer sequência $\left(C_{n}\right)_{n}$ de subconjuntos não vazio fechado, limitados e convexos de $X$ tais que $C_{n+1} \subseteq C_{n}$ para todo $n \in \mathbb{N}$, temos que $\bigcap_{n} C_{n} \neq \emptyset$

Teorema 2.82 [22, pág. 245] Um espaço normado $X$ é reflexivo se, e somente se, a bola unitária $B_{X}$ é fracamente compacta. 
Capítulo 2

\section{Somas torcidas}

Começamos este capítulo dando as definições de soma torcida e sequência exata curta.

Vamos apresentar o método de construção de somas torcidas de Kalton e Peck por meio de funções quase-lineares.

Definição 3.1 (Soma torcida e extensão) Seja $Y$ e $Z$ espaços de Banach. Uma soma torcida de $Y$ e $Z$ é um quase-espaço normado $X$ que contém um espaço $Y^{\prime}$ isomorfo a $Y$ tal que $X / Y^{\prime}$ é isomorfo a Z. Se X é um espaço de Banach, dizemos que X é uma extensão de Y por Z. Quando $Y=Z$ e X é um espaco de Banach, dizemos que X é uma auto-extensão de $Y$.

Definição 3.2 (Sequência exata curta) Sejam Y, Z espaços de Banach e X um quase-espaço de Banach. Um diagrama

$$
0 \rightarrow Y \stackrel{i}{\rightarrow} X \stackrel{j}{\rightarrow} Z \rightarrow 0
$$

onde

- i é linear, contínua e injetora,

- $i(Y)=\operatorname{ker}(j)$,

- jé linear, contínua e sobrejetora

é chamado de sequência exata curta.

A seguinte proposição mostra que uma soma torcida induz uma sequência exata curta.

Proposição 3.3 Seja Y e Z espaços de Banach. O espaço quase-normado X é uma soma torcida de $Y$ e Z se, e somente se, existem funções i e j como na Definição 3.2 tais que

$$
0 \rightarrow Y \stackrel{i}{\rightarrow} X \stackrel{j}{\rightarrow} Z \rightarrow 0
$$

seja uma sequência exata curta. 
Demonstração. Seja $X$ uma soma torcida de $Y$ e $Z$. Seja $Y^{\prime}$ um subespaço de $X$ tal que existem $T: Y^{\prime} \rightarrow Y$ e $S: X / Y^{\prime} \rightarrow Z$ isomorfismos lineares. Se $\pi: X \rightarrow X / Y^{\prime}$ é a aplicação quociente, então

$$
0 \rightarrow Y \stackrel{i=T}{\longrightarrow} X \stackrel{j=S \circ \pi}{\longrightarrow} Z \rightarrow 0
$$

é uma sequência exata curta.

Agora suponha que $0 \rightarrow Y \stackrel{i}{\rightarrow} X \stackrel{j}{\rightarrow} Z \rightarrow 0$ seja uma sequência exata curta. Além disso, $j(X)$ é um subespaço fechado de $X$. O Teorema da Aplicação Aberta implica que $Y$ é isomorfo a $i(Y)$ e $X / i(Y)$ (lembre-se que $\operatorname{ker}(j)=i(Y))$ é isomorfo a $Z$. Pondo $Y^{\prime}=i(Y)$, obtemos que $Y$ é isomorfo $Y^{\prime}$ e $X / Y^{\prime}$ é isomorfo a $Z$, assim $X$ é soma torcida de $Y$ e $Z$.

Proposição 3.4 Uma soma torcida é um espaço quase-Banach.

Demonstração. Sejam $Y, Z$ espaços de Banach e $X$ uma soma torcida de $Y$ e $Z$. Seja

$$
0 \rightarrow Y \stackrel{i=T}{\longrightarrow} X \stackrel{j=S \circ \pi}{\longrightarrow} Z \rightarrow 0
$$

a sequência exata curta definida como na Proposição 3.3. Seja $\left(x_{n}\right)_{n}$ uma sequência de Cauchy em $(X, d)$, onde $d(x, y)=\|\| x-y \|^{p}$ é a métrica definida em Definição 2.51. Vejamos que $\left(x_{n}\right)_{n}$ converge em $(X, d)$. De fato, o Teorema 2.49 garante que $\left\|\left|\|\mid\| \mathrm{e}\|\cdot\|_{X}\right.\right.$ são quase-normas equivalentes, portanto, $\|\cdot\|_{X}$ verifica que dado $\varepsilon>0$, existe $N \in \mathbb{N}$, tal que

$$
\left\|x_{n}-x_{m}\right\|_{X}<\varepsilon,
$$

se $n, m \geq N$. Este fato implica que $j\left(x_{n}\right)$ é uma sequência de Cauchy em $Z$, portanto convergente em $Z$, sem perda de generalidade assumamos que converge para 0 . Note que $j\left(x_{n}\right)=S\left(x_{n}+i(Y)\right)$. Por $S$ ser um isomorfismo, temos que a sequência $\left(x_{n}+i(Y)\right)_{n}$ converge (na quase-norma de $X / i(Y))$ para $i(Y)$, portanto , encontramos uma subsequência de $\left(x_{n}\right)_{n}$, que denotamos por $\left(x_{k_{n}}\right)_{n}$, tal que $\left\|x_{k_{n}}-i\left(y_{n}\right)\right\|_{X} \leq \frac{1}{n^{1 / p}}$ para todo $n \in \mathbb{N}$. Isto implica que $d\left(x_{k_{n}}, i\left(y_{n}\right)\right) \leq \frac{1}{n}$ para todo $n \in \mathbb{N}$ (Teorema de Aoki-Rolewicz). Afirmamos que $i\left(y_{n}\right)$ é uma sequência de Cauchy em $(X, d)$. De fato,

$$
\begin{aligned}
d\left(i\left(y_{n}\right), i\left(y_{m}\right)\right) & \leq d\left(i\left(y_{n}\right), x_{k_{n}}\right)+d\left(i\left(y_{m}\right), x_{k_{m}}\right)+d\left(x_{k_{n}}, x_{k_{m}}\right) \\
& \leq \frac{1}{n}+\frac{1}{m}+d\left(x_{k_{n}}, x_{k_{m}}\right),
\end{aligned}
$$

isto mostra que $i\left(y_{n}\right)$ é uma sequência de Cauchy em $(X, d)$. Agora como $i: Y \longrightarrow i(Y)$ é um isomorfismo entre espaços quase-lineares, demonstramos que $\left(y_{n}\right)$ é uma sequência de Cauchy de $Y$, portanto, converge para algum $y \in Y$. Note que $\left(i\left(y_{n}\right)\right)_{n}$ converge para $i(y)$ em $(X, d)$. 
Pelo fato $d\left(x_{k_{n}}, i\left(y_{n}\right)\right) \leq \frac{1}{n}$ para todo $n \in \mathbb{N}$, demonstramos que $\left(x_{k_{n}}\right)_{n}$ é uma subsequência de $\left(x_{n}\right)_{n}$ convergente, portanto, $\left(x_{n}\right)_{n}$ converge, concluindo assim que $(X, d)$ é um espaço métrico completo.

Definição 3.5 (Sequências exatas curtas equivalentes) Seja $0 \rightarrow Y \stackrel{i}{\rightarrow} X \stackrel{j}{\rightarrow} Z \rightarrow 0 e 0 \rightarrow$ $Y \stackrel{i^{\prime}}{\rightarrow} X^{\prime} \stackrel{j^{\prime}}{\rightarrow} Z \rightarrow 0$ duas sequências exatas curtas. Dizemos que são equivalentes, se existe um operador linear limitado $T: X \longrightarrow X^{\prime}$ tal que o seguinte diagrama comuta

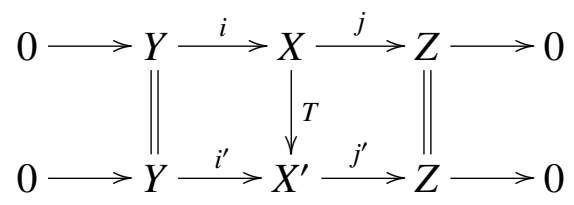

Proposição 3.6 T na Definição 3.5 é um isomorfismo entre espaços quase-normados.

Demonstração. O resultado é imediato da Proposição 2.69.

Definição 3.7 (Somas torcidas equivalentes) Seja $Y$ e $Z$ espaços de Banach e $X, X^{\prime}$ somas torcidas de $Y$ e $Z$. Dizemos que $X$ e $X^{\prime}$ são somas torcidas equivalentes, se existem duas sequências exatas curtas $0 \rightarrow Y \stackrel{i}{\rightarrow} X \stackrel{j}{\rightarrow} Z \rightarrow 0 e 0 \rightarrow Y \stackrel{i^{\prime}}{\rightarrow} X^{\prime} \stackrel{j^{\prime}}{\rightarrow} Z \rightarrow 0$ equivalentes.

Definição 3.8 (Aplicação quase-linear) Sejam $Y, Z$ espaços de Banach e $W$ um espaço vetorial topológico de Hausdorff. Dizemos que $F: Z \rightarrow W$ é uma aplicação quase-linear em $Y$, se $Y$ é subespaço vetorial de $W$, onde a inclusão de $Y$ em $W$ é contínua e $F$ verifica

1. $F(\lambda z)=\lambda F(z)$ para todo $z \in Z$ e $\lambda$ complexo,

2. $F\left(z_{0}+z_{1}\right)-F\left(z_{0}\right)-F\left(z_{1}\right) \in Y$ para todo $z_{0}, z_{1} \in Z$ e existe uma constante $K>0$ tal que

$$
\left\|F\left(z_{0}+z_{1}\right)-F\left(z_{0}\right)-F\left(z_{1}\right)\right\|_{Y} \leq K\left(\left\|z_{0}\right\|_{Z}+\left\|z_{1}\right\|_{Z}\right)
$$

para todo $z_{0}, z_{1} \in Z$. Quando $W=Y$, dizemos que $F: Z \rightarrow Y$ é quase-linear.

O seguinte teorema dá uma forma de construir somas torcidas usando aplicações quase-lineares.

Teorema 3.9 Seja $F: Z \rightarrow W$ uma aplicação quase-linear em Y. Seja

$$
Z \oplus_{F} Y=\{(z, w) \in Z \times W: w-F(z) \in Y\}
$$

e definamos para $(z, w) \in Z \oplus_{F} Y$

$$
\|(z, w)\|_{Z \oplus_{F} Y}=\|z\|_{Z}+\|w-F(z)\|_{Y} .
$$

$O$ espaço $\left(Z \oplus_{F} Y,\|\cdot\|_{Z \oplus_{F} Y}\right)$ é uma soma torcida de $Y$ e $Z$. 
Demonstração. Note que $\|\lambda \cdot(z, w)\|_{Z \oplus_{F} Y}=|\lambda| \cdot\|(z, w)\|_{Z \oplus_{F} Y} \mathrm{e}\|(z, w)\|_{Z \oplus_{F} Y}=0$ se, e somente se, $(z, w)=(0,0)$. Agora

$$
\begin{aligned}
\|(z, w)+(u, v)\|_{Z \oplus_{F} Y}= & \|z+u\|_{Z}+\|w+v-F(z+u)\|_{Y} \\
\leq & \left(\|z\|_{Z}+\|u\|_{Y}\right)+\|w-F(z)\|_{Y}+\|v-F(u)\|_{Y} \\
& +\|F(z)+F(u)-F(z+u)\|_{Y} \\
\leq & \left(\|z\|_{Z}+\|u\|_{Y}\right)+\|w-F(z)\|_{Y}+\|v-F(u)\|_{Y} \\
& +K \cdot\left(\|z\|_{Z}+\|u\|_{Y}\right) \\
\leq & (K+1)\left(\|(z, w)\|_{Z \oplus_{F} Y}+\|(u, v)\|_{Z \oplus_{F} Y}\right)
\end{aligned}
$$

isto mostra que $Z \oplus_{F} Y$ é um espaço quase-normado.

Agora definamos o subespaço $Y_{0}=\{(0, y): y \in Y\}$ de $Z \oplus_{F} Y$. Este subespaço é isométrico a $Y$, pois $\|(0, y)\|_{Z \oplus_{F} Y}=\|y\|_{Y}$. Demonstraremos que $\left(Z \oplus_{F} Y\right) / Y_{0}$ é isométrico a $Z$. De fato,

$$
\begin{aligned}
\left\|(z, w)+Y_{0}\right\|_{\left(Z \oplus_{F} Y\right) / Y^{\prime}} & =\inf \left\{\|(z, w)+(0, v)\|_{Z \oplus_{F} Y}: y \in Y\right\} \\
& =\inf \left\{\|v-F(z)\|_{Y}+\|z\|_{Z}: y \in Y\right\} \\
& =\|z\|_{Z} .
\end{aligned}
$$

Observação 3.10 A sequência exata curta

$$
0 \rightarrow Y \stackrel{i}{\rightarrow} Z \oplus_{F} Y \stackrel{j}{\rightarrow} Z \rightarrow 0
$$

onde $i(y)=(0, y)$ e $j(z, w)=z$, é chamada de sequência exata associada a $Z \oplus_{F} Y$.

Teorema 3.11 Toda soma torcida $X$ de $Y$ e $Z$ é equivalente a $Z \oplus_{F} Y$ para $F: Z \rightarrow Y$ quaselinear oportuna.

Demonstração. Considere a sequência exata curta $0 \rightarrow Y \stackrel{i}{\rightarrow} X \stackrel{j}{\rightarrow} Z \rightarrow 0$. Dado que $j$ é sobrejetora, temos que existe uma aplicação linear $\theta: Z \rightarrow X$ tal que $j \theta=i d_{Z}$ (ver Proposição 2.68) e pela Proposição 2.54 temos que existe uma constante $C>0$ e uma função $\phi: Z \longrightarrow X$ que satisfaz 
- $\|\phi(z)\|_{X} \leq C\|z\|_{Z}$ para todo $z \in Z$,

- $j(\phi(z))=z$, para todo $z \in Z$,

- $\phi(\lambda z)=\lambda \phi(z)$, para todo $z \in Z$ e $\lambda$ escalar.

Os fatos $\phi(z)-\theta(z) \in \operatorname{ker}(j)=\operatorname{Im}(\mathrm{i})$ e $i$ injetora, conduzem a definir $F: Z \rightarrow Y$ por $F(z)=$ $i^{-1}(\phi(z)-\theta(z))$. Seja $\left\|i^{-1}\right\|$ uma constante tal que $\left\|i^{-1}(x)\right\|_{Y} \leq\left\|i^{-1}\right\|\|x\|_{X}$ para todo $x \in \operatorname{Im}(\mathrm{i})$. Para $z_{1}, z_{2} \in Z$

$$
\begin{aligned}
\left\|F\left(z_{1}+z_{2}\right)-F\left(z_{1}\right)-F\left(z_{2}\right)\right\|_{Y} & =\left\|i^{-1}\left(\phi\left(z_{1}+z_{2}\right)-\phi\left(z_{1}\right)-\phi\left(z_{2}\right)\right)\right\|_{Y} \\
& \leq\left\|i^{-1}\right\|\left\|\phi\left(z_{1}+z_{2}\right)-\phi\left(z_{1}\right)-\phi\left(z_{2}\right)\right\|_{X} \\
& \leq\left\|i^{-1}\right\| K_{1}\left(\left\|\phi\left(z_{1}+z_{2}\right)\right\|_{X}+\left\|\phi\left(z_{1}\right)\right\|_{X}+\left\|\phi\left(z_{2}\right)\right\|_{X}\right) \\
& \leq\left\|i^{-1}\right\| K_{1} C\left(\left\|z_{1}+z_{2}\right\|_{Z}+\left\|z_{1}\right\|_{Z}+\left\|z_{2}\right\|_{Z}\right) \\
& \leq 2\left\|i^{-1}\right\| K_{1} C\left(\left\|z_{1}\right\|_{Z}+\left\|z_{2}\right\|_{Z}\right) .
\end{aligned}
$$

$\operatorname{logo}, F$ é uma aplicação quase-linear, pois, $F(\lambda z)=\lambda F(z)$ para todo $\lambda$ escalar.

Definamos um operador $T: X \rightarrow Z \oplus_{F} Y$ por

$$
T(x)=\left(j(x), i^{-1}(x-\theta(j x))\right) .
$$

Demonstraremos que $T$ é contínuo. De fato,

$$
\begin{aligned}
\|T(x)\|_{Z \oplus_{F} Y} & =\|j(x)\|_{Z}+\left\|i^{-1}(x-\theta(j x))-i^{-1}(\phi(j x))-\theta(j x)\right\|_{Y} \\
& =\|j(x)\|_{Z}+\left\|i^{-1}(x-\phi(j x))\right\|_{Y} \\
& \leq\|j(x)\|_{Z}+\left\|i^{-1}\right\|\|x-\phi(j(x))\|_{X} \\
& \leq\|j(x)\|_{Z}+K\left\|i^{-1}\right\|\left(\|x\|_{X}+\|\phi(j(x))\|_{X}\right) \\
& \leq\|j\|\|x\|_{X}+K\left\|i^{-1}\right\|\left(\|x\|_{X}+C\|j\|\|x\|_{X}\right) \\
& =\left(\|j\|+K\left\|i^{-1}\right\|+C K\left\|i^{-1}\right\|\|j\|\right)\|x\|_{X} .
\end{aligned}
$$


Provemos que o seguinte diagrama comuta

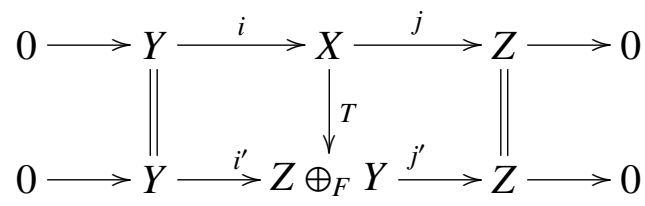

onde $i^{\prime}(y)=(0, y)$ e $j^{\prime}(z, y)=z$. De fato,

$$
\begin{aligned}
T(i(y)) & =\left(j(i(y)), i^{-1}(i(y)-\theta(j(i y))), \text { como } \operatorname{Im}(\mathrm{i})=\operatorname{ker}(\mathrm{j})\right. \text { temos que } \\
& =(0, y) \\
& =i^{\prime}(y) \text { para todo } y \in Y
\end{aligned}
$$

e $j^{\prime} T(x)=j(x)$ para todo $x \in X$.

Definição 3.12 (Aplicações quase-lineares equivalentes) Sejam $F, G: Z \longrightarrow W$ aplicações quase-lineares em $Y$. Dizemos que elas são equivalentes, se existe $M>0$ e uma aplicação linear $A: Z \longrightarrow Y$ tais que $F(z)-G(z)+A(z) \in Y e$

$$
\|F(z)-G(z)+A(z)\|_{Y} \leq M\|z\|_{Z}
$$

para todo $z \in Z$.

Na próxima proposição damos uma condição necessária para que duas aplicações quase-lineares sejam equivalentes.

Proposição 3.13 Se $F, G: Z \longrightarrow W$ são aplicações quase-lineares em $Y$ equivalentes, então $Z \oplus_{F} Y$ e $Z \oplus_{G} Y$ são somas torcidas equivalentes.

Demonstração. Suponha que $F$ e $G$ são funções quase-lineares em $Y$ equivalentes, i.e., existe $M>0$ e uma aplicação linear $A: Z \longrightarrow Y$ tal que $F(z)-G(z)+A(z) \in Y$ e $\|F(z)-G(z)+A(z)\|_{Y} \leq$ $M\|z\|_{Z}$ para todo $z \in Z$. Note que $w-F(z) \in Y$ se, e somente se, $w+A(z)-G(z) \in Y$. Seja $T: Z \oplus_{F} Y \longrightarrow Z \oplus_{G} Y$ definida por $T(z, w)=(z, w+A(z))$

$$
\begin{aligned}
\|T(z, w)\|_{Z \oplus_{G} Y} & =\|z\|_{Z}+\|w+A(z)-G(z)\|_{Y} \\
& \leq\|z\|_{Z}+\|w-F(z)\|_{Y}+\|A(z)+F(z)-G(z)\|_{Y} \\
& \leq(M+1)\|z\|_{Z}+\|w-F(z)\|_{Y} \\
& \leq(M+1)\|(z, w)\|_{Z \oplus_{F} Y}
\end{aligned}
$$


Isto mostra que $T$ é contínuo. Observe que as sequências associadas de $Z \oplus_{F} Y$ e $Z \oplus_{G} Y$ são equivalentes, pois o diagrama

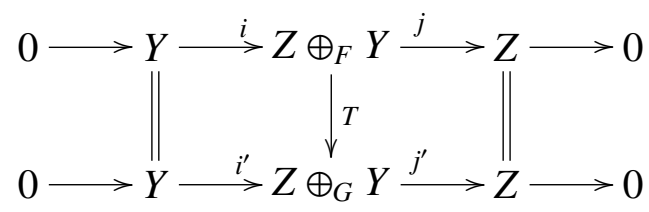

comuta. 
$\Gamma_{\text {Capítulo }}$

\section{Interpolação em espaços de Banach}

Na primeira parte deste capítulo fornecemos algumas definições e resultados da teoria de interpolação (ver [8]).

$\mathrm{Na}$ segunda parte do capítulo abordamos o método de interpolação complexa definido por Calderón em [1]. Provaremos que todo espaço interpolado pode ser fatorado de certa maneita como o producto de dois espaços

\subsection{Generalidades da teoria de interpolação}

Definição 4.1 (Par interpolado) Sejam $X_{0}$ e $X_{1}$ espaços de Banach reais ou complexos. O par $\left[X_{0}, X_{1}\right]$ é chamado de par interpolado, se $X_{0}$ e $X_{1}$ estão mergulhados continuamente em $V$, onde $V$ é um espaço topológico de Hausdorff.

A definição acima permite definir dois subespaços lineares normados de $V$, os quais são: o espaço interseção $X_{0} \cap X_{1}=\left\{v \in V: v \in X_{0}\right.$ e $\left.v \in X_{1}\right\}$ dotado pela norma

$$
\|v\|_{X \cap Y}=\max \left(\|v\|_{X_{0}},\|v\|_{X_{1}}\right)
$$

e o espaço soma $X_{0}+X_{1}=\left\{v \in V\right.$ : existem $x_{0} \in X_{0}$ e $x_{1} \in X_{1}$ tais que $\left.v=x_{0}+x_{1}\right\}$ dotado pela norma

$$
\|v\|_{X_{0}+X_{1}}=\inf \left\{\left\|x_{0}\right\|_{X_{0}}+\left\|x_{1}\right\|_{X_{1}}: v=x_{0}+x_{1}\right\}
$$

Os espaços definidos acima são de Banach e

$$
X_{0} \cap X_{1} \subset X_{0}, X_{1} \subset X_{0}+X_{1} .
$$

Definição 4.2 (Espaço intermédio) Seja $\left[X_{0}, X_{1}\right]$ um par interpolado. Um espaço intermédio entre $X_{0}$ e $X_{1}$, é um espaço de Banach $E$ tal que

$$
X_{0} \cap X_{1} \subset E \subset X_{0}+X_{1} .
$$




\subsection{Interpolação Complexa}

Definição 4.3 (Espaço interpolado) Um espaço interpolado é um espaço intermédio E entre $X_{0}$ e $X_{1}$ com a seguinte propriedade:

se $T: X_{0}+X_{1} \rightarrow X_{0}+X_{1}$ é um operador linear tal que as restrições a $X_{0}$ e $X_{1}$, respectivamente, são $T: X_{0} \rightarrow X_{0}$ e $T: X_{1} \rightarrow X_{1}$, então a restrição de $T$ a $E$ é $T: E \rightarrow E$.

\subsection{Interpolação Complexa}

O método de interpolação complexa desenvolvida por Calderón em [1] será usado ao longo do nosso trabalho.

O seguinte teorema dá condições para que uma função holomorfa de valores em um espaço de Banach verifique o Princípio do Módulo Máximo na faixa $\overline{\mathbb{S}}=\{z \in \mathbb{C}: 0 \leq \operatorname{Re}(z) \leq 1\}$. Nós denotamos o interior de $\overline{\mathbb{S}}$ por $\mathbb{S}$.

Teorema 4.4 (Princípio do Módulo Máximo) Seja $X$ um espaço de Banach complexo e $F$ : $\overline{\mathbb{S}} \longrightarrow X$ uma função holomorfa em $\mathbb{S}$, contínua e limitada . Então

$$
\sup _{z \in \mathbb{S}}\|F(z)\|_{X} \leq \max \left(\sup _{t \in \mathbb{R}}\|F(i t)\|_{X}, \sup _{t \in \mathbb{R}}\|F(1+i t)\|_{X}\right) .
$$

Demonstração. Primeiro provemos o teorema para $X=\mathbb{C}$, assumindo que $F$ verifica $\lim _{|\operatorname{Im}(z)| \rightarrow \infty} F(z)=0$. Consideremos a aplicação $h: \overline{\mathbb{S}} \longrightarrow \mathbb{C}$, definida por

$$
h(z)=\frac{e^{i \pi z}-i}{e^{i \pi z}+i}
$$

A aplicação $h$ é uma bijeção entre $\overline{\mathbb{S}}$ e $\overline{\mathbb{D}}-\{ \pm 1\}$, holomorfa em $\mathbb{S}$ e $h(\partial \overline{\mathbb{S}})=\partial \overline{\mathbb{D}}-\{ \pm 1\}$. Observe que $g(z)=F\left(h^{-1}(z)\right)$ é contínua, limitada em $\overline{\mathbb{D}}-\{ \pm 1\}$ e holomorfa em $\mathbb{D}$. Pelo fato $\lim _{|\operatorname{Im}(z)| \rightarrow \infty} F(z)=0$, obtemos que $\lim _{z \rightarrow \pm 1} g(z)=0$ e portanto estendemos $g$ continuamente a $\overline{\mathbb{D}}$. Aplicando o Princípio do Módulo Máximo no disco temos

$$
|g(z)| \leq \max _{w \in \partial \overline{\mathbb{D}}}|g(w)| \leq \max \left(\sup _{t \in \mathbb{R}}|F(i t)|, \sup _{t \in \mathbb{R}}|F(1+i t)|\right) .
$$

Com isso terminamos a prova para este caso.

Agora seja $F$ uma função qualquer. Definamos $F_{\varepsilon, z_{0}}(z)=e^{\varepsilon\left(z-z_{0}\right)^{2}} F(z)$ onde $\varepsilon>0$ e $z_{0}$ é arbitrário em $\mathbb{S}$. Como $\left|e^{\varepsilon\left(z-z_{0}\right)^{2}}\right| \leq e^{\varepsilon\left(x^{2}-y^{2}\right)} \operatorname{com} z-z_{0}=x+i y \mathrm{e}-1 \leq x \leq 1$, temos $\lim _{|I m(z)| \rightarrow \infty} F_{\varepsilon, z_{0}}(z)=0$. A aplicação $F_{\varepsilon, z_{0}}$ assim definida satisfaz as condições do caso anterior, portanto 


\subsection{Interpolação Complexa}

$$
\begin{aligned}
\left|F\left(z_{0}\right)\right| & =\left|F_{\varepsilon, z_{0}}\left(z_{0}\right)\right| \\
& \leq \max \left(\sup _{t \in \mathbb{R}}\left|F_{\varepsilon, z_{0}}(i t)\right|, \sup _{t \in \mathbb{R}}\left|F_{\varepsilon, z_{0}}(1+i t)\right|\right) \\
& \leq e^{\varepsilon} \max \left(\sup _{t \in \mathbb{R}}|F(i t)|, \sup _{t \in \mathbb{R}}|F(1+i t)|\right)
\end{aligned}
$$

para qualquer $\varepsilon>0$. Como $z_{0}$ foi escolhido arbitrariamente, obtemos

$$
\sup _{z \in \overline{\mathbb{S}}}|F(z)| \leq \max \left(\sup _{t \in \mathbb{R}}|F(i t)|, \sup _{t \in \mathbb{R}}|F(1+i t)|\right) .
$$

Isto mostra o teorema para o caso $X=\mathbb{C}$.

Demonstraremos o caso geral, onde $X$ é um espaço de Bancah complexo. De fato, seja $z_{0} \in \mathbb{S}$ arbitrário. O teorema de Hahn-Banach garante a existência de um funcional linear $\varphi$ de norma 1 em $X$ tal que $\left|\varphi\left(F\left(z_{0}\right)\right)\right|=\left\|F\left(z_{0}\right)\right\|_{X}$. Definamos $g_{\varphi}(z)=\varphi(F(z))$ para $z \in \overline{\mathbb{S}}$. Como $F$ é holomorfa em $\mathbb{S}$, para $w \in \mathbb{S}$, escrevemos $F(z)=\sum_{n=1}^{\infty} a_{n}(z-w)^{n}$, onde $a_{n} \in X$. Então

$$
\begin{aligned}
g_{\varphi}(z) & =\varphi(F(z)) \\
& =\varphi\left(\sum_{n=1}^{\infty} a_{n}(z-w)^{n}\right) \\
& =\sum_{n=1}^{\infty} \varphi\left(a_{n}\right)(z-w)^{n}
\end{aligned}
$$

assim $g_{\varphi}$ é holomorfa em $\mathbb{S}$. Também $g_{\varphi}$ é contínua e limitada, além disso $g_{\varphi}$ verifica as condições do caso $X=\mathbb{C}$, $\log o$

$$
\begin{aligned}
\left\|F\left(z_{0}\right)\right\|_{X} & =\left|g_{\varphi}\left(z_{0}\right)\right| \\
& \leq \max \left(\sup _{t \in \mathbb{R}}\left|g_{\varphi}(i t)\right|, \sup _{t \in \mathbb{R}}\left|g_{\varphi}(1+i t)\right|\right) \\
& \leq \max \left(\sup _{t \in \mathbb{R}}\|F(i t)\|_{X}, \sup _{t \in \mathbb{R}}\|F(1+i t)\|_{X}\right)
\end{aligned}
$$

para $z_{0}$ arbitrário em $\mathbb{S}$. Isto termina a prova do teorema.

Definição 4.5 (Espaço de funções holomorfas) Seja $\left[X_{0}, X_{1}\right]$ um par interpolado. Seja $\overline{\mathbb{S}}=$ $\{z \in \mathbb{C}: 0 \leq \operatorname{Re}(z) \leq 1\}$. Denotemos por $\mathscr{F}$ o conjunto das funções $F: \overline{\mathbb{S}} \rightarrow X_{0}+X_{1}$ tais que 


\subsection{Interpolação Complexa}

1. F é holomorfa em $\mathbb{S}$, contínua e limitado no bordo de $\overline{\mathbb{S}}$.

2. $F($ it $) \in X_{0}$ e $F(1+i t) \in X_{1}$ para todo $t \in \mathbb{R}$.

3. As funções $t \longmapsto F(i t) e t \longmapsto F(1+i t)$ são contínuas $e$

$$
\|F\|_{\mathscr{F}}=\max \left(\sup _{t \in \mathbb{R}}\|F(i t)\|_{X_{0}}, \sup _{t \in \mathbb{R}}\|F(1+i t)\|_{X_{1}}\right)<\infty .
$$

Notemos que o espaço $\mathscr{F}$ assim definido é um espaço vetorial. A expressão que aparece em (3) na Definição 4.5 define uma norma. De fato, se $\|F\|_{\mathscr{F}}=0$, então $\|F(1+i t)\|_{X_{1}}=\|F(i t)\|_{X_{0}}=0$ para todo $t \in \mathbb{R}$. Como

$$
\|F(1+i t)\|_{X_{0}+X_{1}} \leq\|F(1+i t)\|_{X_{1}} \text { e }\|F(i t)\|_{X_{0}+X_{1}} \leq\|F(i t)\|_{X_{0}}
$$

temos que $\|F(i t)\|_{X_{0}+X_{1}}=\|F(1+i t)\|_{X_{0}+X_{1}}=0$ para todo $t \in \mathbb{R}$, assim o Teorema 4.4 implica que $F=0$. É imediato provar que $\|\lambda F\|_{\mathscr{F}}=|\lambda|\|F\|_{\mathscr{F}}$ para $\lambda \in \mathbb{C}$. A desigualdade triangular é uma consequência da igualdade de números positivos

$$
\max (a+b, c+d)=\max (a, c)+\max (b, d) .
$$

De fato,

$$
\begin{aligned}
\|F+G\|_{\mathscr{F}} & =\max \left(\sup _{t \in \mathbb{R}}\|(F+G)(i t)\|_{X_{0}}, \sup _{t \in \mathbb{R}}\|(F+G)(1+i t)\|_{X_{1}}\right) \\
& \leq \max \left(\sup _{t \in \mathbb{R}}\|F(i t)\|_{X_{0}}+\sup _{t \in \mathbb{R}}\|G(i t)\|_{X_{0}}, \sup _{t \in \mathbb{R}}\|F(1+i t)\|_{X_{1}}+\sup _{t \in \mathbb{R}}\|G(1+i t)\|_{X_{1}}\right) \\
& =\|F\|_{\mathscr{F}}+\|G\|_{\mathscr{F}} .
\end{aligned}
$$

Observação 4.6 Vamos dar algumas observações em relação a $\left(\mathscr{F},\|\cdot\|_{\mathscr{F}}\right)$

- Algumas vezes denotamos o espaço $\mathscr{F}$ por $\mathscr{F}\left(X_{0}, X_{1}\right)$ para indicar que as aplicações de $\mathscr{F}$ são definidas de $\overline{\mathbb{S}}$ em $X_{0}+X_{1}$.

- Se $\varphi: \mathbb{S} \longrightarrow \mathbb{D}$ é uma aplicação conforme que pode ser estendida continuamente a $\partial \overline{\mathbb{S}}$, então $\|\varphi F\|_{\mathscr{F}}=\|F\|_{\mathscr{F}}$, pois $\varphi(\partial \overline{\mathbb{S}})=\partial \overline{\mathbb{D}}-\{ \pm 1\} e$

$$
\begin{aligned}
\|\varphi F\|_{\mathscr{F}} & =\max \left(\sup _{t \in \mathbb{R}}\|\varphi(i t) F(i t)\|_{X_{0}}, \sup _{t \in \mathbb{R}}\|\varphi(i t+1) F(1+i t)\|_{X_{1}}\right) \\
& =\max \left(\sup _{t \in \mathbb{R}}|\varphi(i t)|\|F(i t)\|_{X_{0}}, \sup _{t \in \mathbb{R}}|\varphi(i t+1)|\|F(1+i t)\|_{X_{1}}\right) \\
& =\max \left(\sup _{t \in \mathbb{R}}\|F(i t)\|_{X_{0}}, \sup _{t \in \mathbb{R}}\|F(1+i t)\|_{X_{1}}\right) \\
& =\|F\|_{\mathscr{F}} .
\end{aligned}
$$




\subsection{Interpolação Complexa}

Teorema 4.7 $\mathscr{F}$ é um espaço de Banach.

Demonstração. Suponha que $\left(F_{n}\right)_{n}$ seja uma sequência de Cauchy em $\mathscr{F}$. Pelo Teorema 4.4 temos para cada $z \in \overline{\mathbb{S}}$

$$
\begin{aligned}
\left\|F_{n}(z)-F_{m}(z)\right\|_{X_{0}+X_{1}} & \leq \max \left(\sup _{t \in \mathbb{R}}\left\|\left(F_{n}-F_{m}\right)(i t)\right\|_{X_{0}+X_{1}}, \sup _{t \in \mathbb{R}}\left\|\left(F_{n}-F_{m}\right)(1+i t)\right\|_{X_{0}+X_{1}}\right) \\
& \leq \max \left(\sup _{t \in \mathbb{R}}\left\|\left(F_{n}-F_{m}\right)(i t)\right\|_{X_{0}}, \sup _{t \in \mathbb{R}}\left\|\left(F_{n}-F_{m}\right)(1+i t)\right\|_{X_{1}}\right) \\
& =\left\|F_{n}-F_{m}\right\|_{\mathscr{F}}
\end{aligned}
$$

por $X_{0}+X_{1}$ ser de Banach, temos que $F_{n}(z)$ converge para algum $F(z) \in X_{0}+X_{1}$.

Demonstraremos que a sequência $\left(F_{n}\right)_{n}$ converge uniformemente em norma de $X_{0}+X_{1}$ para $F$. De fato, seja $z \in \mathbb{S}$. Então existe $N(z) \in \mathbb{N}$, que depende de $z$, tal que

$$
n \geq N(z) \Rightarrow\left\|F_{n}(z)-F(z)\right\|_{X_{0}+X_{1}}<\varepsilon .
$$

Também existe $N \in \mathbb{N}$ satisfazendo

$$
n, m \geq N \Rightarrow\left\|F_{n}(z)-F_{m}(z)\right\|_{X_{0}+X_{1}}<\varepsilon,
$$

para qualquer $z$. Agora

$$
\begin{aligned}
\left\|F_{n}(z)-F(z)\right\|_{X_{0}+X_{1}} & \leq\left\|F_{n}(z)-F_{m}(z)\right\|_{X_{0}+X_{1}}+\left\|F(z)-F_{m}(z)\right\|_{X_{0}+X_{1}} \\
& <\left\|F_{n}(z)-F_{m}(z)\right\|_{X_{0}+X_{1}}+\varepsilon \text { se } m \geq N(z), N \\
& <2 \varepsilon \text { se } n \geq N .
\end{aligned}
$$

Isto mostra que $\left(F_{n}\right)_{n}$ converge uniformemente em norma de $X_{0}+X_{1}$ para $F$.

A Proposição 2.28 garante que $F$ é holomorfa em $\mathbb{S}$. Como $F_{n}(i t) \in X_{0}$ e $F_{n}(1+i t) \in X_{1}$ convergem uniformemente em norma de $X_{0}+X_{1}$ para $F(i t)$ e $F(1+i t)$ respectivamente, então devido a que $X_{0}$ e $X_{1}$ são espaços de Banach temos que $F(i t) \in X_{0}$ e $F(1+i t) \in X_{1}$.

Provaremos a continuidade da função $t \longmapsto F(i t)$. Seja $t_{0}$ real fixo e $\varepsilon>0$. Então existe $N \in \mathbb{N}$ tal que $\left\|F_{N}(i t)-F(i t)\right\|_{X_{0}}<\varepsilon$ para qualquer $t$. Por $t \longmapsto F_{N}(i t)$ ser contínua, temos que para $\varepsilon>0$ existe $\delta>0$ tal que

$$
\left|t-t_{0}\right|<\delta \Rightarrow\left\|F_{N}(i t)-F_{N}\left(i t_{0}\right)\right\|_{X_{0}}<\varepsilon
$$




\subsection{Interpolação Complexa}

usando este fato temos

$$
\begin{aligned}
\left\|F(i t)-F\left(i t_{0}\right)\right\|_{X_{0}} & \leq\left\|F\left(i t_{0}\right)-F_{N}\left(i t_{0}\right)\right\|_{X_{0}}+\left\|F_{N}(i t)-F(i t)\right\|_{X_{0}}+\left\|F_{N}\left(i t_{0}\right)-F_{N}(i t)\right\|_{X_{0}} \\
& <\varepsilon+\varepsilon+\left\|F_{N}\left(i t_{0}\right)-F_{N}(i t)\right\|_{X_{0}} . \\
& <3 \varepsilon \text { sempre que }\left|t-t_{0}\right|<\delta .
\end{aligned}
$$

Isto termina a prova da continuidade de $t \longmapsto F(i t)$. Similarmente provamos que a função $t \longmapsto F(+1 i t)$ é contínua.

Como $\left(F_{n}\right)_{n}$ é uma sequência de funções limitadas no bordo que converge uniformemente em norma para $F$, temos que $F$ é limitada no bordo, assim $F \in \mathscr{F}$.

Definição 4.8 Seja $\left[X_{0}, X_{1}\right]$ um par interpolado. Seja $0<\theta<1$. Definimos um subespaço linear $X_{\theta}$ de $X_{0}+X_{1}$ por

$$
X_{\theta}=\left\{x \in X_{0}+X_{1}: x=F(\theta) \text { para algum } F \in \mathscr{F}\right\} .
$$

O subespaço $X_{\theta}$ é denotado por $\left[X_{0} X_{1}\right]_{\theta}$.

A expressão $\|x\|_{X_{\theta}}=\inf \left\{\|F\|_{\mathscr{F}}: x=F(\theta)\right\}$ é uma norma no espaço $X_{\theta}$.

Proposição 4.9 $X_{\theta}$ é um espaço intermédio.

Demonstração. Primeiro demonstraremos que se $0<\theta<1$, então

$$
X_{0} \cap X_{1} \subseteq X_{\theta} \subseteq X_{0}+X_{1}
$$

De fato, seja $x \in X_{0} \cap X_{1}$ e considere a função constante $F(z)=x$ para todo $z \in \mathbb{S}$. Note que $F \in \mathscr{F}$, portanto, $x \in X_{\theta}$ e $\|x\|_{X_{\theta}} \leq\|F\|_{\mathscr{F}}=\max \left(\|x\|_{X_{0}},\|x\|_{X_{1}}\right)=\|x\|_{X_{0} \cap X_{1}}$ e $\operatorname{assim} X_{0} \cap X_{1} \subseteq X_{\theta}$. Provemos que $X_{\theta} \subseteq X_{0}+X_{1}$. Suponha que $x \in X_{\theta}$ e $x=F(\theta)$. Então pelo Princípio do Módulo Máximo temos

$$
\begin{aligned}
\|x\|_{X_{0}+X_{1}} & \leq \max \left(\sup _{t \in \mathbb{R}}\|F(i t)\|_{X_{0}+X_{1}}, \sup _{t \in \mathbb{R}}\|F(1+i t)\|_{X_{0}+X_{1}}\right) \\
& \leq \max \left(\sup _{t \in \mathbb{R}}\|F(i t)\|_{X_{0}}, \sup _{t \in \mathbb{R}}\|F(1+i t)\|_{X_{1}}\right) \\
& =\|F\|_{\mathscr{F}},
\end{aligned}
$$

$\operatorname{assim}\|x\|_{X_{0}+X_{1}} \leq\|x\|_{X_{\theta}}$.

Para terminar a prova da proposição, basta demonstrar que $X_{\theta}$ é Banach. Consideremos uma função $\delta_{\theta}: \mathscr{F} \longrightarrow X_{0}+X_{1}$ definida por $\delta_{\theta}(F)=F(\theta)$. Como $\|F(\theta)\|_{X_{0}+X_{1}} \leq\|F\| \|_{\mathscr{F}}$, temos que 


\subsection{Interpolação Complexa}

$\delta_{\theta}$ é contínuo, portanto $\mathscr{N}=\operatorname{ker}\left(\delta_{\theta}\right)=\{F \in \mathscr{F}: F(\theta)=0\}$ é fechado, além disso $\mathscr{F} / \mathscr{N}$ é de Banach. Consideremos $C>1$ e $F \in \mathscr{F}$. Então existe $G \in \mathscr{F}$ tal que $G(\theta)=F(\theta)$ e $\|G\|_{\mathscr{F}} \leq C\|F(\theta)\|_{X_{\theta}}$, isto é, $\|G\|_{\mathscr{F}} \leq C\left\|\delta_{\theta}(F)\right\|_{X_{\theta}}$, portanto $\delta_{\theta}$ verifica (3) do Teorema 2.70, assim $\delta_{\theta}(\mathscr{F})=X_{\theta}$ é isomorfo a $\mathscr{F} / \mathscr{N}$.

O seguinte teorema mostra que $X_{\theta}$ é um espaço interpolado entre $X_{0}$ e $X_{1}$.

Teorema 4.10 Seja $\left[X_{0}, X_{1}\right]$ e $\left[Y_{0}, Y_{1}\right]$ pares interpolados. Seja $T: X_{0}+X_{1} \longrightarrow Y_{0}+Y_{1} u m$ operador linear contínuo tal que $T$ seja contínuo de $X_{i}$ em $Y_{i}$ para $i=0,1$. Então $T$ é um operador contínuo de $X_{\theta}$ em $Y_{\theta}$ e sua norma, que denotamos por $\|T\|_{\theta}$, verifica

$$
\|T\|_{\theta} \leq\|T\|_{0}^{1-\theta}\|T\|_{1}^{\theta}
$$

onde $\|T\|_{i}$ denota a norma de $T: X_{i} \longrightarrow Y_{i}$, para $i=0,1$.

Demonstração. Denotemos por $\|T\|_{i}$ a norma do operador $T: X_{i} \longrightarrow Y_{i}$ para $i=0,1$. Primeiro assumamos que as normas são não nulas. Seja $x \in X_{\theta}$. Então existe $F \in \mathscr{F}\left(X_{0}, X_{1}\right)$ tal que $F(\theta)=x$. Definamos uma função $G$ no conjunto $\mathbb{S}$ por

$$
G(z)=\left(\frac{\|T\|_{0}}{\|T\|_{1}}\right)^{z-\theta} T(F(z)) .
$$

Demonstraremos que $G \in \mathscr{F}\left(Y_{0}, Y_{1}\right)$. De fato, como $T: X_{0} \longrightarrow Y_{0}$ e $F(i t) \in X_{0}$, então $T(F(i t)) \in Y_{0}$, portanto $G(i t) \in Y_{0}$. Do mesmo modo mostramos que $G(1+i t) \in Y_{1}$. Como $T$ é contínua e a função $t \longmapsto F(i t)$ é contínua, obtemos que $t \longmapsto G(i t)$ é contínua. Analogamente demonstramos que a função $t \longmapsto G(1+i t)$ é contínua. Vejamos que $G$ é holomorfa $\mathbb{S}$. Como $F$ é holomorfa em $\mathbb{S}$, para $w \in \mathbb{S}$, escrevemos $F(z)=\sum_{n=1}^{\infty} a_{n}(z-w)^{n}$, onde $a_{n} \in X_{0}+X_{1}$, portanto

$$
\begin{aligned}
G(z) & =\left(\sum_{m=1}^{\infty} \zeta_{m}(z-w)^{m}\right) T(F(z)) \\
& =\left(\sum_{m=1}^{\infty} \zeta_{m}(z-w)^{m}\right) T\left(\sum_{n=1}^{\infty} a_{n}(z-w)^{n}\right) \\
& =\left(\sum_{m=1}^{\infty} \zeta_{m}(z-w)^{m}\right) \sum_{n=1}^{\infty} T\left(a_{n}\right)(z-w)^{n} \\
& =\sum_{m=1}^{\infty} \sum_{n=1}^{\infty} \zeta_{m} T\left(a_{n}\right)(z-w)^{n+m},
\end{aligned}
$$

onde

$$
\left(\frac{\|T\|_{0}}{\|T\|_{1}}\right)^{z-\theta}=\sum_{m=1}^{\infty} \zeta_{m}(z-w)^{m}
$$




\subsection{Interpolação Complexa}

e $\zeta_{m} \in \mathbb{C}$, assim $G$ é holomorfa em $\mathbb{S}$. Agora

$$
\begin{aligned}
\|G(i t)\|_{Y_{0}} & =\left\|\left(\frac{\|T\|_{0}}{\|T\|_{1}}\right)^{i t-\theta} T(F(i t))\right\|_{Y_{0}} \\
& =\left(\frac{\|T\|_{0}}{\|T\|_{1}}\right)^{-\theta}\|T(F(i t))\|_{Y_{0}} \\
& \leq\|T\|_{0}^{1-\theta}\|T\|_{1}^{\theta}\|F(i t)\|_{X_{0}} .
\end{aligned}
$$

Similarmente mostramos que

$$
\|G(1+i t)\|_{Y_{1}} \leq\|T\|_{0}^{1-\theta}\|T\|_{1}^{\theta}\|F(1+i t)\|_{X_{1}} .
$$

Isto prova que $G$ é limitado no bordo de $\overline{\mathbb{S}}$ e além disso

$$
\|G\|_{\mathscr{F}\left(Y_{0}, Y_{1}\right)} \leq\|T\|_{0}^{1-\theta}\|T\|_{1}^{\theta}\|F\|_{\mathscr{F}\left(X_{0}, X_{1}\right)} .
$$

Observe que $T(x)=T(F(\theta))=G(\theta)$ e assim

$$
\|T(x)\|_{Y_{\theta}} \leq\|G\|_{\mathscr{F}\left(Y_{0}, Y_{1}\right)} \leq\|T\|_{0}^{1-\theta}\|T\|_{1}^{\theta}\|F\|_{\mathscr{F}\left(X_{0}, X_{1}\right)},
$$

tomando o ínfimo sobre os $F \in \mathscr{F}\left(X_{0}, X_{1}\right)$ tais que $x=F(\theta)$, deduzimos

$$
\|T(x)\|_{Y_{\theta}} \leq\|T\|_{0}^{1-\theta}\|T\|_{1}^{\theta}\|x\|_{X_{\theta}} .
$$

Agora, suponha sem perda de generalidade que $\|T\|_{0} \neq 0$ e $\|T\|_{1}=0$. Definamos para $\varepsilon>0$

$$
G(z)=\left(\frac{\|T\|_{0}}{\varepsilon}\right)^{z-\theta} T(F(z))
$$

De uma maneira similar ao caso anterior provamos que $G \in \mathscr{F}\left(Y_{0}, Y_{1}\right), G(\theta)=T(x)$,

$$
\|G(i t)\|_{Y_{0}}=\|T\|_{0}^{1-\theta} \varepsilon^{\theta}\|F(i t)\|_{X_{0}}
$$

$\mathrm{e}$

$$
\|G(1+i t)\|_{Y_{1}}=0
$$

assim,

$$
\|G\|_{\mathscr{F}\left(Y_{0}, Y_{1}\right)}=\|T\|_{0}^{1-\theta} \varepsilon^{\theta}\|F\|_{\mathscr{F}\left(X_{0}, X_{1}\right)},
$$

$\log 0$

$$
\|T(x)\|_{Y_{\theta}} \leq\|G\|_{\mathscr{F}\left(Y_{0}, Y_{1}\right)} \leq\|T\|_{0}^{1-\theta} \varepsilon^{\theta}\|F\|_{\mathscr{F}\left(X_{0}, X_{1}\right)}
$$

para qualquer $\varepsilon>0$ o que implica $T=0$, e portanto limitado.

Se $\|T\|_{0}=\|T\|_{1}=0$, defina $G(z)=T(F(z))$. Como $G(\theta)=T(x)$ e $G(i t)=G(1+i t)=0$ temos que $G=0$, portanto $T=0$ e assim limitada.

Agora vamos dar um exemplo de espaço interpolado de Calderón. 


\subsection{Interpolação Complexa}

Exemplo 4.11 (Interpolação de espaços $L_{p}$ ) Seja $\mu$ uma medida $\sigma$-finita em $S$ e consideremos os espaços $L_{p_{0}}$ e $L_{p_{1}}$ mergulhados em $L_{p_{0}}+L_{p_{1}}$. Se $0<\theta<1$ e $1 \leq p_{0}, p_{1} \leq \infty$, então $\left[L_{p_{0}}, L_{p_{1}}\right]_{\theta}=L_{p}$, onde $p$ verifica

$$
\frac{1}{p}=\frac{1-\theta}{p_{0}}+\frac{\theta}{p_{1}}
$$

De fato, seja $f$ uma função de soporte compacto. Sem perda de generalidade assumamos que $\|f\|_{L_{p}}=1$. Definamos uma função em $F: \overline{\mathbb{S}} \longrightarrow L_{p_{0}}+L_{p_{1}}$ por

$$
F(z)(s)= \begin{cases}|f(s)|^{p\left(\frac{1-z}{p_{0}}+\frac{z}{p_{1}}\right)} \cdot \frac{f(s)}{|f(s)|} & \text { se } s \in S, f(s) \neq 0 . \\ 0 & \text { se } s \in S, f(s)=0 .\end{cases}
$$

A função $F$ é holomorfa em $\mathbb{S}$ e contínua em $\overline{\mathbb{S}}$, com

$$
\begin{gathered}
|F(i t)(s)|=|f(s)|^{p / p_{0}}, \text { se } f(s) \neq 0 \\
|F(1+i t)(s)|=|f(s)|^{p / p_{1}}, \text { se } f(s) \neq 0,
\end{gathered}
$$

isto é, $F(i t) \in L_{p_{0}}$ e $F(1+i t) \in L_{p_{1}}$, com

$$
\begin{gathered}
\|F(i t)\|_{L_{p_{0}}} \leq\|f\|_{L_{p}}^{p / p_{0}}=1, \\
\|F(1+i t)\|_{L_{p_{1}}} \leq\|f\|_{L_{p}}^{p / p_{1}}=1 .
\end{gathered}
$$

Também as funções $t \in \mathbb{R} \longmapsto F(i t)$ e $t \in \mathbb{R} \longmapsto F(1+i t)$ são contínuas, portanto, $F \in \mathscr{F}\left(L_{p_{0}}, L_{p_{1}}\right)$, com $\|F\|_{\mathscr{F}\left(L_{p_{0}} L_{p_{1}}\right)} \leq 1$. Como $F(\theta)=f$, obtemos que $f \in\left[L_{p_{0}}, L_{p_{1}}\right]_{\theta}$ e $\|f\|_{\left[L_{p_{0}}, L_{p_{1}}\right]_{\theta}} \leq 1=\|f\|_{L_{p}}$. Isto prova que $L_{p} \subset\left[L_{p_{0}}, L_{p_{1}}\right]_{\theta}$.

Demostraremos que $\left[L_{p_{0}}, L_{p_{1}}\right]_{\theta} \subset L_{p}$. De fato, seja $f \in\left[L_{p_{0}}, L_{p_{1}}\right]_{\theta}$ e sem perda de generalidade suponha que $1=\|f\|_{\left[L_{p_{0}}, L_{p_{1}}\right]_{\theta}}$. Então existe $F \in \mathscr{F}\left(L_{p_{0}}, L_{p_{1}}\right)$ tal que $f=F(\theta)$. Sejam $q, q_{0}$ e $q_{1}$ os conjugados de $p, p_{0}$ e $p_{1}$, respectivamente, e $y$ uma função simples, com $\|y\|_{L_{q}}=1$. Definamos uma função $G$ em $\overline{\mathbb{S}}$ por

$$
G(z)(s)= \begin{cases}|y(s)|^{q\left(\frac{1-z}{q_{0}}+\frac{z}{q_{1}}\right)} \cdot \frac{y(s)}{|y(s)|} & \text { se } s \in S, y(s) \neq 0 . \\ 0 & \text { se } s \in S, y(s)=0 .\end{cases}
$$

Note que $G$ assim definida é holomorfa em $\mathbb{S}$ verifica $G(i t) \in L_{q_{0}}$ e $G(1+i t) \in L_{q_{1}}$, com

$$
\|G(i t)\|_{L_{q_{0}}} \leq\|y\|_{L_{q}}^{q / q_{0}}=1
$$




\subsection{Interpolação Complexa}

$$
\|G(1+i t)\|_{L_{q_{1}}} \leq\|y\|_{L_{q}}^{q / q_{1}}=1
$$

Definamos outra função $H$ em $\overline{\mathbb{S}}$ de valor complexo por

$$
H(z)=\int_{S} F(z)(s) G(z)(s) d \mu .
$$

Por $F$ e $G$ ser funções holomorfas em $\mathbb{S}$, obtemos que a função $H$ é holomorfa em $\mathbb{S}$. Também a função $H$ é contínua e limitada em $\overline{\mathbb{S}}$. Então pelo Teorema 4.4 obtemos que

$$
|H(z)| \leq \max \left(\sup _{t \in \mathbb{R}}|H(i t)|, \sup _{t \in \mathbb{R}}|H(1+i t)|\right) \text { para todo } z \in \overline{\mathbb{S}} .
$$

A desigualdade de Hölder implica que

$$
|H(i t)| \leq\|F(i t)\|_{L_{p_{0}}} \cdot\|G(i t)\|_{L_{q_{0}}} \leq\|F(i t)\|_{L_{p_{0}}} \cdot\|y\|_{L_{q}}^{q / q_{0}}=\|F(i t)\|_{L_{p_{0}}} .
$$

Similarmente

$$
|H(1+i t)| \leq\|F(1+i t)\|_{L_{p_{1}}} \cdot\|G(1+i t)\|_{L_{q_{1}}} \leq\|F(1+i t)\|_{L_{p_{1}}} \cdot\|y\|_{L_{q}}^{q / q_{1}}=\|F(1+i t)\|_{L_{p_{1}}},
$$

isto é,

$$
|H(z)| \leq \max \left(\sup _{t \in \mathbb{R}}\|F(i t)\|_{L_{p_{0}}}, \sup _{t \in \mathbb{R}}\|F(1+i t)\|_{L_{p_{1}}}\right)=\|F\|_{\mathscr{F}\left(L_{p_{0}} L_{p_{1}}\right)}, \text { para todo } z \in \overline{\mathbb{S}},
$$

em particular para $z=\theta$, temos que

$$
\left|\int_{S} f(s) y(s) d \mu\right|=|H(\theta)| \leq\|F\|_{\mathscr{F}\left(L_{p_{0}} L_{p_{1}}\right)},
$$

para toda função simples $y$ em $L_{q} \operatorname{com}\|y\|_{L_{q}}=1$, portanto, $f \in L_{p} \mathrm{e}$

$$
\|f\|_{L_{p}}=\sup \left\{\left|\int_{S} f(s) y(s) d \mu\right|: y \text { função simples com }\|y\|_{L_{q}}=1\right\} \leq\|F\|_{\mathscr{F}\left(L_{p_{0}} L_{p_{1}}\right)},
$$

como $F$ foi escolhida arbitrariamente em $\mathscr{F}\left(L_{p_{0}} L_{p_{1}}\right)$ com $F(\theta)=f$, obtemos que $\|f\|_{L_{p}} \leq\|f\|_{\left[L_{p_{0}}, L_{p_{1}}\right]_{\theta}}$. Assim temos que $\left[L_{p_{0}}, L_{p_{1}}\right]_{\theta} \subset L_{p}$ e portanto $\left[L_{p_{0}}, L_{p_{1}}\right]_{\theta}=L_{p}$.

Se aplicamos o Teorema 4.10 ao Exemplo 4.11, obtemos um resultado interessante:

Teorema 4.12 (Teorema de Riesz-Thorin) Seja $\mu$ uma medida $\sigma$-finita sobre $S$ e $0<\theta<1$. Se $T: L_{p_{0}}+L_{p_{1}} \longrightarrow L_{q_{0}}+L_{q_{1}}$ é um operador linear limitado tal que

$$
T: L_{p_{0}} \longrightarrow L_{q_{0}} \text { e } T: L_{p_{1}} \longrightarrow L_{q_{1}}
$$




\subsection{Interpolação Complexa}

são operadores limitados, com normas respectivas $\|T\|_{0} e\|T\|_{1}$, então a restrição de $T$ ao espaço $L_{p}$, com

$$
\frac{1}{p}=\frac{1-\theta}{p_{0}}+\frac{\theta}{p_{1}}
$$

é limitada e

$$
T: L_{p} \longrightarrow L_{q}
$$

onde

$$
\frac{1}{q}=\frac{1-\theta}{q_{0}}+\frac{\theta}{q_{1}} .
$$

A norma da restrição de $T$ a $L_{p}$, que denotamos por $\|T\|_{p}$, é estimada por

$$
\|T\|_{p} \leq\|T\|_{0}^{1-\theta} \cdot\|T\|_{1}^{\theta} .
$$

Definição 4.13 Sejam $X_{0}, X_{1}$ espaços de Köthe e $0<\theta<1$. Definamos um espaço de Banach por

$$
X_{0}^{1-\theta} X_{1}^{\theta}=\left\{x \in L_{0}: \text { existem } u_{0} \in X_{0}, u_{1} \in X_{1} \text {, tais que }|x|=\left|u_{0}\right|^{1-\theta}\left|u_{1}\right|^{\theta}\right\},
$$

normado por

$$
\|x\|=\inf \left\{\left\|u_{0}\right\|_{X_{0}}^{1-\theta} \cdot\left\|u_{1}\right\|_{X_{1}}^{\theta}:|x|=\left|u_{0}\right|^{1-\theta}\left|u_{1}\right|^{\theta}\right\} .
$$

Note que espaço $X_{0}^{1-\theta} X_{1}^{\theta}$ pode ser escrito como

$$
X_{0}^{1-\theta} X_{1}^{\theta}=\left\{x \in L_{0}: \text { existem } u_{0} \in B_{X_{0}}, u_{1} \in B_{X_{1}}, \lambda \geq 0 \text { tais que }|x| \leq \lambda\left|u_{0}\right|^{1-\theta}\left|u_{1}\right|^{\theta}\right\}
$$

com norma

$$
\|x\|=\inf \left\{\lambda: \text { existem } u_{0} \in B_{X_{0}}, u_{1} \in B_{X_{1}} \text { tais que }|x| \leq \lambda\left|u_{0}\right|^{1-\theta}\left|u_{1}\right|^{\theta}\right\} .
$$

Vamos comentar a prova de un resultado apresentado por Calderón em [1].

Teorema 4.14 Sejam $X_{0}$ e $X_{1}$ espaços de Banach de funções, tais que ou $X_{0}$ ou $X_{1}$ é reflexivo. Então

$$
X_{\theta}=X_{0}^{1-\theta} X_{1}^{\theta}
$$

onde a igualdade é no sentido isométrico.

Demonstração. Vamos provar o teorema em partes:

Passo 1. Vamos estabelecer algumas notações e definições .

Seja $X$ um espaco de Banach de funções (ver Definição 2.37) e $B$ um espaço de Banach. Uma função $f: S \longrightarrow B$ é chamada de função com B-valores. Denotamos por $X(B)$, o conjunto de 


\subsection{Interpolação Complexa}

todas as funções com $B$-valores $f$ tais que $\|f(\cdot)\|_{B} \in X$. Note que o conjunto $X(B)$ é um espaço de Banach, com

$$
\|f\|_{X(B)}=\|\| f(\cdot)\left\|_{B}\right\|_{X} .
$$

Sejam $X_{0}, X_{1}$ espaços de Banach de funções de valores reais contidos em $L_{0}(S, \mu)$ e seja $\left[B_{0}, B_{1}\right]$ um par interpolado. Denotamos por $\mathscr{F}_{0}\left(X_{0}\left(B_{0}\right), X_{1}\left(B_{1}\right)\right)$ o subconjunto de $\mathscr{F}\left(X_{0}\left(B_{0}\right), X_{1}\left(B_{1}\right)\right)$ de todas as funções $F$ da forma

$$
F(s, \xi)=e^{\delta \xi^{2}} \sum_{k=1}^{N} e^{\lambda_{n} \xi} f(s)
$$

onde as $f_{n}$ são funções em $X_{0}\left(B_{0}\right) \cap X_{1}\left(B_{1}\right), \lambda_{n}$ são números reais, $\delta>0, s \in S$ e $\xi \in \overline{\mathbb{S}}$. O subconjunto $\mathscr{F}_{0}\left(X_{0}\left(B_{0}\right), X_{1}\left(B_{1}\right)\right)$ é denso em $\mathscr{F}\left(X_{0}\left(B_{0}\right), X_{1}\left(B_{1}\right)\right)$. Definamos outro espaço de funções , que denotamos por $\mathscr{G}\left(Y_{0}, Y_{1}\right)$, como o conjunto das funções $G: \overline{\mathbb{S}} \longrightarrow Y_{0}+Y_{1}$ tais que

1. $G$ é holomorfa em $\mathbb{S}$ e contínua no bordo $\overline{\mathbb{S}}$.

2. $\|G(z)\|_{Y_{0}+Y_{1}} \leq c(1+|z|)$, para alguma constante $c>0$.

3. A função $G$ verifica $G\left(i t_{1}\right)-G\left(i t_{2}\right) \in Y_{0}, G\left(1+i t_{1}\right)-G\left(1+i t_{2}\right) \in Y_{1}$ para todo $t_{1}, t_{2} \in \mathbb{R} \mathrm{e}$

$$
\|G\|_{\mathscr{G}}=\max \left(\sup _{t_{1}, t_{2} \in \mathbb{R}}\left\|\frac{G\left(i t_{1}\right)-G\left(i t_{2}\right)}{t_{1}-t_{2}}\right\|_{Y_{0}}, \sup _{t_{1}, t_{2} \in \mathbb{R}}\left\|\frac{G\left(1+i t_{1}\right)-G\left(1+i t_{2}\right)}{t_{1}-t_{2}}\right\|_{Y_{1}}\right)<\infty .
$$

O espaço $X_{[\theta]}=\left\{G^{\prime}(\theta): G \in \mathscr{G}\left(Y_{0}, Y_{1}\right)\right\}$ munido da norma

$$
\|x\|_{X_{[\theta]}}=\inf \left\{\|G\|_{\mathscr{G}}: G^{\prime}(\theta)=x\right\}
$$

define um espaço interpolado entre $Y_{0}$ e $Y_{1}$. Também denotamos o espaço $X_{[\theta]}$ por $\left[X_{0} X_{1}\right]_{[\theta]}$.

Vamos denotar $X=X_{0}^{1-\theta} X_{1}^{\theta}$ e $B=\left[B_{0}, B_{1}\right]_{\theta}$.

Vamos provar o resultado para espaços de funções com $B$-valores.

Passo 2. Os espaços interpolados verificam

$$
\left[X_{0}\left(B_{0}\right), X\left(B_{1}\right)\right]_{\theta} \subset X(B) \subset\left[X_{0}\left(B_{0}\right), X\left(B_{1}\right)\right]_{[\theta]},
$$

onde as inclusões são contínuas. 


\subsection{Interpolação Complexa}

Passo 2.A. Demostremos que $\left[X_{0}\left(B_{0}\right), X\left(B_{1}\right)\right]_{\theta} \subset X(B)$. De fato, consideremos uma função em $\mathscr{F}\left(X_{0}\left(B_{0}\right), X_{1}\left(B_{1}\right)\right)$ da forma

$$
F(s, \xi)=e^{\delta \xi^{2}} \sum_{k=1}^{N} e^{\lambda_{n} \xi} f(s),
$$

onde as $f_{n}$ são funções em $X_{0}\left(B_{0}\right) \cap X_{1}\left(B_{1}\right), \lambda_{n}$ são números reais, $\delta>0, s \in S$ e $\xi \in \overline{\mathbb{S}}$. As $f_{n}$ são funções com $B$-valores, pois $B_{0} \cap B_{1} \subset B$. Agora damos uma estimativa da norma de $F(s, \xi)$, demonstrando as desigualdades seguintes:

$$
\begin{gathered}
\cdot \log \|F(s, \theta)\|_{B} \leq \int_{-\infty}^{+\infty} \rho_{0}(\theta, t) \log \|F(s, i t)\|_{B_{0}} d t+\int_{-\infty}^{+\infty} \log \|F(s, 1+i t)\|_{B_{1}} \rho_{1}(\theta, t) d t \\
\bullet\|F(s, \theta)\|_{B} \leq\left(\frac{1}{1-\theta} \int_{-\infty}^{+\infty}\|F(s, i t)\|_{B_{0}} \rho_{0}(\theta, t) d \mu\right)^{1-\theta} \cdot\left(\frac{1}{\theta} \int_{-\infty}^{+\infty}\|F(s, 1+i t)\|_{B_{1}} \rho_{1}(\theta, t) d t\right)^{\theta}
\end{gathered}
$$

onde

$$
\rho_{0}(\xi, t)=\frac{e^{-\pi(t-r)} \sin \pi \tau}{\sin ^{2} \pi \tau+\left(\cos \pi \tau-e^{-\pi(t-r)}\right)^{2}}, \text { onde } \xi=\tau+i r
$$

$\mathrm{e}$

$$
\rho_{1}(\xi, t)=\frac{e^{-\pi(t-r)} \sin \pi \tau}{\sin ^{2} \pi \tau+\left(\cos \pi \tau+e^{-\pi(t-r)}\right)^{2}}, \text { onde } \xi=\tau+i r
$$

são os kernel de Poisson (ver [23]).

Demonstraremos a primeira desigualdade. De fato, seja $M \in \mathbb{N}$ e $\varepsilon>0$. Calderón encontrou duas funções $\varphi_{0, n, \varepsilon}$ e $\varphi_{1, n, \varepsilon}$ infinitamente diferenciáveis em $\mathbb{R}$ tais que $\lim _{|t| \rightarrow \infty} \varphi_{j, n, \varepsilon}(t)=-n+\varepsilon$ e

$$
\max \left(\log \|F(\cdot, j+i t)\|_{B_{j}},-n\right) \leq \varphi_{j, n, \varepsilon}(t) \leq \max \left(\log \|F(\cdot, j+i t)\|_{B_{j}},-n\right)+2 \varepsilon
$$

para todo $t \in \mathbb{R}$ e $j=0,1$. Ele demonstrou que existe uma função analítica, limitada e contínua em $\overline{\mathbb{S}}$, que denotamos por $\phi$, tal que $\operatorname{Re}(\phi(i t))=\varphi_{0, n, \varepsilon}(t), \operatorname{Re}(\phi(1+i t))=\varphi_{1, n, \varepsilon}(t)$ e

$$
\operatorname{Re}(\phi(\xi))=\int_{-\infty}^{+\infty} \rho_{0}(\xi, t) \varphi_{0, n, \varepsilon}(t) d t+\int_{-\infty}^{+\infty} \rho_{1}(\xi, t) \varphi_{1, n, \varepsilon}(t) d t .
$$

Note que 


\subsection{Interpolação Complexa}

$$
\begin{aligned}
\left\|e^{-\phi(i t)} F(s, j+i t)\right\|_{B_{j}} & =e^{\operatorname{Re}(-\phi(j+i t))} \cdot\|F(s, j+i t)\|_{B_{j}} \\
& =e^{-\varphi_{j, n, \varepsilon}(t)} \cdot\|F(s, j+i t)\|_{B_{j}} \text { pela desigualdade }
\end{aligned}
$$

(4.1) temos que

$\leq 1$

para $j=0,1$ e todo $s \in S$. Isto implica que $e^{-\phi(\cdot)} F(s, \cdot) \in \mathscr{F}\left(B_{0}, B_{1}\right) \mathrm{e}$

$$
\left\|e^{-\phi(\cdot)} F(s, \cdot)\right\|_{\mathscr{F}\left(B_{0}, B_{1}\right)} \leq 1,
$$

portanto,

$$
\|F(s, \theta)\|_{B} \leq e^{\operatorname{Re}(\phi(\theta))},
$$

que equivale a

$$
\log \|F(s, \theta)\|_{B} \leq \operatorname{Re}(\phi(\theta))=\int_{-\infty}^{+\infty} \rho_{0}(\theta, t) \varphi_{0, n, \varepsilon}(t) d t+\int_{-\infty}^{+\infty} \rho_{1, n, \varepsilon}(\theta, t) \varphi_{1}(t) d t .
$$

Se $n \rightarrow \infty$ e $\varepsilon \rightarrow 0$, obtemos pela desigualdade (4.1) que

$$
\log \|F(s, \theta)\|_{B} \leq \int_{-\infty}^{+\infty} \rho_{0}(\theta, t) \log \|F(s, i t)\|_{B_{0}} d t+\int_{-\infty}^{+\infty} \log \|F(s, 1+i t)\|_{B_{1}} \rho_{1}(\theta, t) d t .
$$

Isto mostra a primeira desigualdade.

Agora demonstraremos a segunda desigualdade. De fato, o kernel de Poisson verifica que

$$
\int_{-\infty}^{+\infty} \rho_{0}(\theta, t) d t=1-\theta \text { e } \int_{-\infty}^{+\infty} \rho_{1}(\theta, t) d t=\theta
$$

portanto, pela desigualdade de Jensen (ver Corolário 2.56) obtemos que

$$
\exp \left(\frac{1}{1-\theta} \int_{-\infty}^{+\infty} \rho_{0}(\theta, t) \log \|F(s, i t)\|_{B_{0}} d t\right) \leq \frac{1}{1-\theta} \int_{-\infty}^{+\infty} \rho_{0}(\theta, t)\|F(s, i t)\|_{B_{0}} d t
$$

e

$$
\exp \left(\frac{1}{\theta} \int_{-\infty}^{+\infty} \rho_{1}(\theta, t) \log \|F(s, 1+i t)\|_{B_{1}} d t\right) \leq \frac{1}{\theta} \int_{-\infty}^{+\infty} \rho_{1}(\theta, t)\|F(s, 1+i t)\|_{B_{1}} d t .
$$

Aplicando logaritmo nas duas desigualdades acimas, multiplicado a primeira por $(1-\theta)$ e a segunda por $\theta$, somando as desigualdades obtidas e por último aplicando (4.2), temos que $\log \|F(s, \theta)\|_{B} \leq \log \left[\left(\frac{1}{1-\theta} \int_{-\infty}^{+\infty} \rho_{0}(\theta, t)\|F(s, i t)\|_{B_{0}} d t\right)^{1-\theta}\left(\frac{1}{\theta} \int_{-\infty}^{+\infty} \rho_{1}(\theta, t)\|F(s, 1+i t)\|_{B_{1}} d t\right)^{\theta}\right]$, 


\subsection{Interpolação Complexa}

demonstrando assim que

$$
\|F(s, \theta)\|_{B} \leq\left(\frac{1}{1-\theta} \int_{-\infty}^{+\infty}\|F(s, i t)\|_{B_{0}} \rho_{0}(\theta, t) d \mu\right)^{1-\theta} \cdot\left(\frac{1}{\theta} \int_{-\infty}^{+\infty}\|F(s, 1+i t)\|_{B_{1}} \rho_{1}(\theta, t) d t\right)^{\theta} .
$$

Se definirmos

$$
g(s)=\frac{1}{1-\theta} \int_{\infty}^{-\infty}\|F(s, i t)\|_{B_{0}} \rho_{0}(\theta, t) d \mu,
$$

e

$$
h(s)=\frac{1}{\theta} \int_{\infty}^{-\infty}\|F(s, 1+i t)\|_{B_{1}} \rho_{1}(\theta, t) d t,
$$

temos que $g \in X_{0}$ e $h \in X_{1}$, pois $\|F(\cdot, i t)\|_{B_{0}} \in X_{0}$ e $\|F(\cdot, 1+i t)\|_{B_{1}} \in X_{1}$, resumindo

$$
\|F(s, \theta)\|_{B} \leq g(s)^{1-\theta} h(s)^{\theta},
$$

para todo $s \in S$. Por $X$ ser um espaço de Banach de funções, obtemos que $\|F(\cdot, \theta)\|_{B} \in X$ e $\|g\|_{X_{0}},\|h\|_{X_{1}} \leq\|F\|_{\mathscr{F}}$, assim

$$
\|\| F(\cdot, \theta)\left\|_{B}\right\|_{X} \leq\left\|g^{1-\theta} h^{\theta}\right\|_{X}
$$

$\log 0$

$$
\|F(\theta)\|_{X(B)} \leq\|F\|_{\mathscr{F}}, \text { se } F \in \mathscr{F}_{0}\left(X_{0}\left(B_{0}\right) X_{1}\left(B_{1}\right)\right) .
$$

Seja $x \in\left[X_{0}\left(B_{0}\right), X\left(B_{1}\right)\right]_{\theta}$. Então, existe $F \in \mathscr{F}\left(X_{0}\left(B_{0}\right) X_{1}\left(B_{1}\right)\right)$ tal que $x=F(\theta)$ com $\|F\|_{\mathscr{F}} \leq\|x\|_{\theta}+\varepsilon$. Por $\mathscr{F}_{0}\left(X_{0}\left(B_{0}\right) X_{1}\left(B_{1}\right)\right)$ ser denso em $\mathscr{F}\left(X_{0}\left(B_{0}\right) X_{1}\left(B_{1}\right)\right)$, temos que existe uma sequência $\left(F_{n}\right)_{n}$ em $\mathscr{F}_{0}\left(X_{0}\left(B_{0}\right) X_{1}\left(B_{1}\right)\right)$ tal que $F_{n} \rightarrow F$ em $\|\cdot\|_{\mathscr{F}}$. Além disso,

$$
\left\|F_{n}(\theta)-F(\theta)\right\|_{\theta} \leq\left\|F_{n}-F\right\|_{\mathscr{F}} \rightarrow 0, \text { se } n \rightarrow \infty .
$$

Por outro lado,

$$
\left\|F_{n}(\theta)-F_{m}(\theta)\right\|_{X(B)} \leq\left\|F_{n}-F_{m}\right\|_{\mathscr{F}},
$$

isto prova que $\left(F_{n}(\theta)\right)_{n}$ é uma sequência de Cauchy em $X(B)$, portanto, converge em $X(B)$, assim $F(\theta) \in X(B)$. Também

$$
\left\|F_{n}(\theta)\right\|_{X(B)} \leq\left\|F_{n}\right\|_{\mathscr{F}}
$$

e se $n \rightarrow \infty$, obtemos

$$
\|F(\theta)\|_{X(B)} \leq\|F\|_{\mathscr{F}} \leq\|x\|_{\theta}+\varepsilon .
$$

Isto demonstra que

$$
\|x\|_{X(B)} \leq\|x\|_{\theta}+\varepsilon,
$$




\subsection{Interpolação Complexa}

para $\varepsilon$ arbitrário, logo $\|x\|_{X(B)} \leq\|x\|_{\theta}$. Em conclusão $\left[X_{0}\left(B_{0}\right), X\left(B_{1}\right)\right]_{\theta} \subset X(B)$.

Passo 2.B. Demonstraremos que $X(B) \subset\left[X_{0}\left(B_{0}\right), X\left(B_{1}\right)\right]_{[\theta]}$. De fato, seja $f \in X(B)$ uma função cuja imagem seja um conjunto enumerável, isto é, $f$ toma os valores $u_{1}, \ldots, u_{n}, \ldots \in B$. Escolhamos $g \in X_{0}$ e $h \in X_{1}$, com $\|g\|_{X_{0}},\|h\|_{X_{1}} \leq 1 \mathrm{e}$

$$
\|f(\cdot)\|_{B}=\|f\|_{X(B)}(1+\varepsilon) g(\cdot)^{1-\theta} h(\cdot)^{\theta} .
$$

Seja $S_{j}=\left\{s \in S: f(s)=u_{j}\right\}$. Como $u_{j} \in B=\left[B_{0} B_{1}\right]_{\theta}$, então existe $\varphi \in \mathscr{F}\left(B_{0}, B_{1}\right)$, com $\left\|\varphi_{j}\right\|_{\mathscr{F}} \leq 1+\varepsilon$, tal que $\varphi(\theta)=\frac{u_{j}}{\left\|u_{j}\right\|_{B}}$. Definamos uma função $F$ por

$$
F(s, \xi)= \begin{cases}\|f\|_{X(B)}(1+\varepsilon) g(s)^{1-\xi} h(s)^{\xi} \sum_{j=1}^{\infty} 1_{S_{j}}(s) \cdot \varphi(\xi) & \text { se } h(s) g(s) \neq 0, \\ 0 & \operatorname{se} h(s) g(s)=0\end{cases}
$$

Seja $\Gamma$ um caminho contido na faixa $\{z: 0 \leq \operatorname{Re}(z) \leq 1\}$ que liga os pontos $1 / 2$ e $\xi$. Definamos uma função $F_{1}(s, \xi)=\int_{\Gamma} F(s, \eta) d \eta$. A definição de $F_{1}$ não depende da escolha do caminho $\Gamma$. A função $F_{1}$ pertence a $\mathscr{F}\left(X_{0}\left(B_{0}\right), X_{1}\left(B_{1}\right)\right)$ e $\left\|F_{1}\right\|_{\mathscr{F}} \leq(1+\varepsilon)\|f\|_{X(B)}$. Também satisfaz que

$$
\frac{d}{d \xi} F_{1}(s, \theta)=F(s, \theta)=f(s)
$$

portanto, $f \in\left[X_{0}\left(B_{0}\right) X\left(B_{1}\right)\right]_{[\theta]}$ e $\|f\|_{[\theta]} \leq(1+\varepsilon)\|f\|_{X(B)}$, como $\varepsilon$ é arbitrário, obtemos que $\|f\|_{[\theta]} \leq\|f\|_{X(B)}$. Resumindo, se $f \in X(B)$ tem imagem enumerável, então $f \in$ $\mathscr{F}\left(X_{0}\left(B_{0}\right), X_{1}\left(B_{1}\right)\right)$ e $\|f\|_{[\theta]} \leq\|f\|_{X(B)}$.

Vamos demonstrar o caso geral. Seja $f \in X(B)$ arbitrário. Como o conjunto das funções em $X(B)$ com imagem enumerável é denso em $X(B)$, então existe uma sequência de funções $\left(f_{n}\right)_{n}$ com imagem enumerável tal que $\left\|f_{n}-f\right\|_{X(B)} \rightarrow 0$, se $n \rightarrow \infty$. Note que $f_{n}-f_{m} \in$ $\left[X_{0}\left(B_{0}\right), X_{1}\left(B_{1}\right)\right]_{[\theta]}$, com $\left\|f_{n}-f\right\|_{[\theta]} \leq\left\|f_{n}-f\right\|_{X(B)}$. Isto prova que $\left(f_{n}\right)_{n}$ é uma sequência de Cauchy em $\left[X_{0}\left(B_{0}\right), X_{1}\left(B_{1}\right)\right]_{[\theta]}$, portanto, convergente em $\left[X_{0}\left(B_{0},\right) X_{1}\left(B_{1}\right)\right]_{[\theta]}$, isto é, $f \in$ $\left[X_{0}\left(B_{0}\right), X_{1}\left(B_{1}\right)\right]_{[\theta]}$. Agora

$$
\|f\|_{[\theta]}=\lim _{n \rightarrow \infty}\left\|f_{n}\right\|_{[\theta]} \leq \lim _{n \rightarrow \infty}\left\|f_{n}\right\|_{X(B)}=\|f\|_{X(B)} .
$$

Em conclusão

$$
X(B) \subset\left[X_{0}\left(B_{0}\right), X\left(B_{1}\right)\right]_{[\theta]} .
$$

Passo 3. Vejamos que se ou $X_{0}\left(B_{0}\right)$ ou $X_{1}\left(B_{1}\right)$ é reflexivo, então $X(B)=\left[X_{0}\left(B_{0}\right), X\left(B_{1}\right)\right]_{\theta}$. Basta provar que $\left[X_{0}\left(B_{0}\right), X\left(B_{1}\right)\right]_{[\theta]} \subset\left[X_{0}\left(B_{0}\right), X\left(B_{1}\right)\right]_{\theta}$.

Denotemos por $X_{0}\left(B_{0}\right)=Y_{0}$ e $X_{1}\left(B_{1}\right)=Y_{1}$. Neste passo usamos o seguinte lema provado pelo Calderón em [1, pág. 118]. 


\subsection{Interpolação Complexa}

Lema 4.15 Se $F \in \mathscr{G}\left(Y_{0}, Y_{1}\right) e$

$$
\frac{F(i t+i h)-F(i t)}{h}
$$

converge em $Y_{0}$ em um conjunto de medida positiva quando $h \rightarrow 0$ ( $h$ real $)$, então $F^{\prime}(\theta) \in$ $\left[Y_{0}, Y_{1}\right]_{\theta}=Y_{\theta}$, para todo $0<\theta<1$.

Suponha que $Y_{0}$ é reflexivo. Seja $F \in \mathscr{G}\left(Y_{0}, Y_{1}\right)$. Então $p(t)=F(i t)$ é uma função contínua em relação a $t$, portanto, a imagem de $p$ está contida em um subespaço separável de $Y_{0}$ (tome o espaço gerado pela imagem de $p$ ), que denotamos por $V$. Sejam

$$
F_{n}(z)=\left(F\left(z+\frac{i}{n}\right)-F(z)\right) \cdot \frac{n}{i}
$$

$R_{m}(t)$ o fecho fraco do conjunto $\left\{F_{n}(i t): n \geq m\right\}$ e $R(t)=\bigcap_{m \in \mathbb{N}} R_{m}(t)$. Para todo $t \in \mathbb{R}$, os conjuntos $R_{m}(t)$ e $R(t)$ estão contidos em um conjunto limitado de $Y_{0}$, pois como $F \in \mathscr{G}\left(Y_{0}, Y_{1}\right)$, obtemos que

$$
\begin{aligned}
\left\|F_{n}(i t)\right\|_{Y_{0}} & =\left\|F\left(i\left(t+\frac{1}{n}\right)\right)-F(i t)\right\|_{Y_{0}} \cdot n \\
& \leq\|F\|_{\mathscr{G}}\left|t+\frac{1}{n}-t\right| \cdot n \\
& =\|F\|_{\mathscr{G}}, \text { para todo } n \in \mathbb{N} .
\end{aligned}
$$

Agora como $R_{m}(t)$ é um conjunto limitado, então existe uma bola centrada em zero, que denotamos por $D_{0}$, tal que $R_{m}(t) \subset D_{0}$, pelo fato de $Y_{0}$ ser reflexivo, obtemos que $D_{0}$ é fracamente compacta (ver Teorema 2.82). Devido a que $R_{m}(t)$ é fracamente fechado e está contido em um conjunto fracamente compacto, então $R_{m}(t)$ é fracamente compacto, portanto, convexo e limitado. O teorema da interseção de Cantor (ver Teorema 2.81) implica que $R(t)$ é não vazio. Seja $g$ uma função tal que $g(t) \in R(t)$ para todo $t \in \mathbb{R}$. Note que $R(t) \subset V$, logo a imagem de $g$ é separável.

Demonstraremos que

$$
F(i t)=F(0)+i \int_{0}^{t} g(\tau) d \tau
$$

Seja $L$ um funcional linear contínuo em $Y_{0}$ e $\varphi(t)=-i L(F(i t))$. Como $F \in \mathscr{G}\left(Y_{0}, Y_{1}\right)$, então $\varphi$ é uma função de Lipschitz. Agora

$$
L\left(F_{n}(i t)\right)=n\left(\varphi\left(t+\frac{1}{n}\right)-\varphi(t)\right) .
$$

Note que a imagem do conjunto $R_{m}(t)$ pelo operador $L$ é o fecho fraco do conjunto

$$
\left\{n\left(\varphi\left(t+\frac{1}{n}\right)-\varphi(t)\right): n \geq m\right\}
$$




\subsection{Interpolação Complexa}

portanto, a imagem de $R(t)$ por $L$ é a interseção dos conjuntos acimas. Como $\varphi$ é uma função de Lipschitz, então $\varphi$ é quase-sempre diferenciável em $\mathbb{R}$ (ver Teorema 2.32), isto é, existe um conjunto de medida zero $A$, tal que $\varphi^{\prime}(t)$ existe para todo $t \in \mathbb{R} \backslash A$, e $\varphi^{\prime}$ é fortemente mensurável. Isto equivale a dizer que $L(g(t))$ existe quase-sempre e é fortemente mesurável, em consequência $g$ é fracamente mensurável. Devido a que a imagem de $g$ é separável, da Observação 2.24 deduzimos que $g$ é fortemente mensurável. Lembre-se que a imagem de $g$ está contida em $R(t)$, portanto, $g$ é uma função limitada, em particular, limitada em qualquer intervalo finito real, assim o Teorema 2.26 garante que $g$ é Bochner integrável em todo intervalo finito real. Note que

$$
L(F(i t))=i \varphi(t)=i \varphi(0)+i \int_{0}^{t} \varphi^{\prime}(\tau) d \tau=L(F(0))+i \int_{0}^{t} L(g(\tau)) d \tau,
$$

para todo $L$ operador linear contínuo em $Y_{0}$, assim

$$
F(i t)=F(0)+i \int_{0}^{t} g(\tau) d \tau
$$

Da igualdade acima temos que $F(i t)$ é quase-sempre diferenciável em relação a $t$, portanto,

$$
\frac{1}{h} \cdot \frac{F(i t+i h)-F(i t)}{h} \rightarrow 0
$$

quando $h \rightarrow 0$ em um conjunto de medida positiva de $\mathbb{R}$. Daqui deduzimos que $F^{\prime}(\theta) \in$ $\left[Y_{0}, Y_{1}\right]_{[\theta]}$. Vejamos que a inclusão é contínua. De fato, seja $x \in X_{[\theta]}$, isto é, existe $F \in \mathscr{G}\left(Y_{0}, Y_{1}\right)$ tal que $x=F^{\prime}(\theta)$ e $\|F\|_{\mathscr{G}} \leq\|x\|_{[\theta]}+\varepsilon$. Consideremos a função

$$
h_{n}(z)=e^{\varepsilon z^{2}} F_{n}(z) \text {, }
$$

onde

$$
F_{n}(z)=\left(F\left(z+\frac{i}{n}\right)-F(z)\right) \cdot \frac{n}{i} .
$$

Observe que $h_{n} \in \mathscr{F}\left(Y_{0}, Y_{1}\right)$ e

$$
\begin{aligned}
\left\|h_{n}(i t)\right\|_{Y_{0}} & =\left\|e^{-\varepsilon t^{2}} F_{n}(i t)\right\|_{Y_{0}} \\
& =n \cdot e^{-\varepsilon t^{2}}\left\|F\left(i t+\frac{i}{n}\right)-F(i t)\right\|_{Y_{0}} \\
& \leq e^{-\varepsilon t^{2}}\left\|F\left(i t+\frac{i}{n}\right)-F(i t)\right\|_{Y_{0}} \\
& \leq\|F\|_{\mathscr{G}} .
\end{aligned}
$$




\subsection{Interpolação Complexa}

Similarmente provamos que $\left\|h_{n}(1+i t)\right\|_{Y_{1}} \leq e^{\varepsilon}\|F\|_{\mathscr{G}}$, portanto,

$$
\begin{aligned}
\left\|h_{n}\right\|_{\mathscr{F}} & \leq\left(1+e^{\varepsilon}\right)\|F\|_{\mathscr{G}} \\
& \leq\left(1+e^{\varepsilon}\right)\left(\|x\|_{[\theta]}+\varepsilon\right),
\end{aligned}
$$

$\operatorname{logo}$

$$
\left\|h_{n}(\theta)\right\|_{\theta} \leq\left(1+e^{\varepsilon}\right)\left(\|x\|_{[\theta]}+\varepsilon\right) .
$$

Agora

$$
\begin{aligned}
\left\|h_{n}(\theta)-e^{\varepsilon \theta^{2}} x\right\|_{\theta}= & e^{\varepsilon \theta^{2}}\left\|\left(F\left(\theta+\frac{i}{n}\right)-F(\theta)\right) \cdot \frac{n}{i}-F^{\prime}(\theta)\right\|_{\theta} \\
= & e^{\varepsilon \theta^{2}}\left\|\frac{F\left(\theta+\lambda_{n}\right)-F(\theta)}{\lambda_{n}}-F^{\prime}(\theta)\right\|_{\theta} \text {, onde } \lambda_{n}=\frac{i}{n} \\
& \rightarrow 0, \text { se } n \rightarrow \infty .
\end{aligned}
$$

Então

$$
\begin{aligned}
\|x\|_{\theta} & =\left\|h_{n}(\theta) e^{-\varepsilon \theta^{2}}-h_{n}(\theta) e^{-\varepsilon \theta^{2}}+e^{-\varepsilon \theta^{2}} \cdot e^{\varepsilon \theta^{2}} x\right\|_{\theta} \\
\leq & e^{-\varepsilon \theta^{2}}\left\|h_{n}(\theta)\right\|_{\theta}+e^{-\varepsilon \theta^{2}}\left\|h_{n}(\theta)-e^{\varepsilon \theta^{2}} x\right\|_{\theta} \\
\leq & e^{-\varepsilon \theta^{2}}\left(1+e^{\varepsilon}\right)\left(\|x\|_{[\theta]}+\varepsilon\right)+e^{-\varepsilon \theta^{2}}\left\|h_{n}(\theta)-e^{\varepsilon \theta^{2}} x\right\|_{\theta} \\
& \rightarrow e^{-\varepsilon \theta^{2}}\left(1+e^{\varepsilon}\right)\left(\|x\|_{[\theta]}+\varepsilon\right), \text { se } n \rightarrow \infty .
\end{aligned}
$$

Se $\varepsilon \rightarrow 0$, então $\|x\|_{\theta} \leq\|x\|_{[\theta]}$, portanto, a inclusão é contínua. Em conclusão $\left[Y_{0}, Y_{1}\right]_{[\theta]} \subset\left[Y_{0}, Y_{1}\right]_{\theta}$.

Neste trabalho, nós sempre vamos assumir que $X_{\theta}=X_{0}^{1-\theta} X_{1}^{\theta}$. 


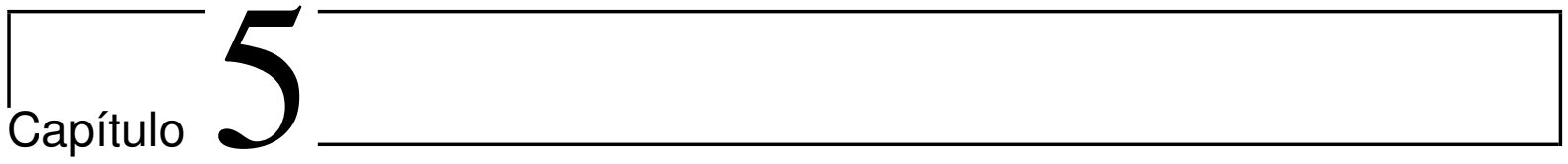

\section{Espaços de Köthe e espaços de funções $g$-convexos}

Definição 5.1 (Espaço admissível) Seja $S$ um espaço polonês e $\mu$ uma medida $\sigma$-finita de Borel em $S$. Dizemos que a função $f \longmapsto\|f\|_{X}\left(L_{0} \longmapsto[0, \infty]\right)$ é uma norma admissível, se o conjunto $X=\left\{x \in L_{0}:\|x\|_{X}<\infty\right\}$ verifica as seguintes condições :

1. $\left(X,\|\cdot\|_{X}\right)$ é um espaço normado.

2. $B_{X}=\left\{x:\|x\|_{X} \leq 1\right\}$ é fechado em $L_{0}$ e contém um elemento positivo.

3. Existe h positivo em $L_{0}$ tal que

$$
\|h x\|_{L_{1}} \leq\|x\|_{X}
$$

para todo $x \in L_{0}$.

O espaço X assim definido é chamado de espaço admissível.

Definição 5.2 (Espaço de Köthe) Um espaço admissivel é chamado de espaço de Köthe se $|x(s)| \leq|y(s)|$ para todo $s \in S$ com $x$ mensurável e $y \in X$, então $x \in X$ e $\|x\|_{X} \leq\|y\|_{X}$.

Observação 5.3 Se uma norma (ou quase-norma) verifica esta propriedade, então dizemos que a norma (ou quase-norma) $\|\cdot\|_{X}$ preserva ordem.

Definição 5.4 (Espaço de funções $g$-convexo ) Seja $x \longmapsto\|x\|_{X}\left(L_{0} \longmapsto[0, \infty]\right)$ uma função e $X=\left\{x \in L_{0}:\|x\|_{X}<\infty\right\}$. O espaço $X$ é chamado de espaço de funções g-convexo, se verifica:

1. $B_{X}=\left\{x:\|x\|_{X} \leq 1\right\}$ é fechado em $L_{0}$ e contém um elemento positivo.

2. $\|\alpha \cdot x\|_{X}=|\alpha| \cdot\|x\|_{X}$ para todo $x \in X$ e todo $\alpha \in \mathbb{C}$. 
3. Para todo $x, u, v \in L_{0} e 0 \leq \theta \leq 1$, com $|x| \leq|u|^{1-\theta}|v|^{\theta}$, temos que $\|x\|_{X} \leq\|u\|_{X}^{1-\theta}\|v\|_{X}^{\theta}$.

4. Para cada $0<\tau<\infty$, existe um número $N(\tau)$, com $\lim _{\tau \rightarrow 0} N(\tau)=0$, tal que, se $x, y \in X$, com $\operatorname{supp}(x) \cap \operatorname{supp}(y)=\emptyset e\|x\|_{X} \leq 1,\|y\|_{X} \leq \tau$, então $\|x+y\|_{X} \leq 1+N(\tau)$. Denotemos o menor número $N(\tau) \geq 0$ que verifica as condições acima por $\eta(\tau)$.

Observação 5.5 Seja X um espaço de funções g-convexo.

1. Se $\theta=0$ e $x, u \in X$ em 3 na definição acima, temos que $|x| \leq|u|$ implica $\|x\|_{X} \leq\|u\|_{X}$.

2. Um espaço de Köthe X é um espaço de funções g-convexo. De fato:

Note que $X$ satisfaz 1. e 2. da Definição 5.4, portanto, basta apenas provar 3 e 4. Para demonstrar 3 , nós usamos a desigualdade

$$
a^{\theta} b^{1-\theta} \leq \theta a+(1-\theta) b
$$

onde $a, b \geq 0$ e $0 \leq \theta \leq 1$,

$$
\begin{aligned}
|u|^{\theta}|v|^{1-\theta} & =\left|c^{1 / \theta} u\right|^{\theta} \cdot\left|c^{-1 /(1-\theta)} v\right|^{1-\theta} \\
& \leq \theta \cdot\left|c^{1 / \theta} u\right|+(1-\theta) \cdot\left|c^{-1 /(1-\theta)} v\right|,
\end{aligned}
$$

para todo $u, v \in L_{0}$ e $c \geq 0$, o que implica

$$
\left\||u|^{\theta}|v|^{1-\theta}\right\|_{X} \leq \theta c^{1 / \theta} \cdot\|u\|_{X}+(1-\theta) c^{1 /(1-\theta)} \cdot\|v\|_{X} .
$$

Em particular, se $c=\left(\|v\|_{X} /\|u\|_{X}\right)^{\theta(1-\theta)}$, obtemos que

$$
\left\||u|^{\theta} \cdot|v|^{1-\theta}\right\|_{X} \leq\|u\|_{X}^{\theta} \cdot\|v\|_{X}^{1-\theta} .
$$

Agora, supondo que $|x| \leq|u|^{\theta}|v|^{1-\theta}$, temos imediatamente que $\|x\|_{X} \leq\|u\|_{X}^{\theta} \cdot\|v\|_{X}^{1-\theta}$.

Demonstraremos que $X$ verifica a parte 4. da Definição 5.4. Sejam $0<\tau<\infty$ e $x, y \in X$, com $\operatorname{supp}(x) \cap \operatorname{supp}(y)=\emptyset \mathrm{e}\|x\|_{X} \leq 1,\|y\|_{X} \leq \tau$. Por $\|\cdot\|_{X}$ ser uma norma, temos que $\|x+y\|_{X} \leq$ $\|x\|_{X}+\|y\|_{X} \leq 1+\tau$ e pela definição de $\eta(\tau)$ (ver Definição 5.4, parte 4.), segue que $0 \leq \eta(\tau) \leq \tau$, $\operatorname{assim} \lim _{\tau \rightarrow 0} \eta(\tau)=0$.

Observação 5.6 Se X é um espaço g-convexo, então toda função $f$ de $X$, é quase-finita, isto é, o conjunto dos elementos $E=\{s: f(s)=\infty\}$ tem medida zero. De fato:

Se $f \in X$, então $\|f\|_{X}<\infty$. Observe que $n \cdot\left|1_{E}\right| \leq|f|$ para todo $n \in \mathbb{N}$, portanto, $n \cdot\left|1_{E}\right| \in X$ e $n \cdot\left\|1_{E}\right\|_{X} \leq\|f\|_{X}$ para todo $n \in \mathbb{N}$, assim $1_{E}=0$, isto é, $\mu(E)=0$. 
Proposição 5.7 Todo espaço g-convexo é um espaço quase-normado de Banach. Em particular, todo espaço de Köthe é um espaço de Banach.

Demonstração. Vejamos que $X$ é um espaço quase-normado. De fato, sejam $x, y \in X$ e definamos duas funções como

$$
x_{0}(s)= \begin{cases}x(s)+y(s) & \text { se }|x(s)| \leq|y(s)| \\ 0 & \text { se }|x(s)|>|y(s)|\end{cases}
$$

e,

$$
y_{0}(s)= \begin{cases}x(s)+y(s) & \text { se }|x(s)|>|y(s)|, \\ 0 & \text { se }|x(s)| \leq|y(s)| .\end{cases}
$$

Observe que $\operatorname{supp}\left(x_{0}\right) \cap \operatorname{supp}\left(y_{0}\right)=\emptyset$ e $x_{0}+y_{0}=x+y$.

Agora pela parte 4. da Definição 5.4 temos

$$
\left\|\frac{x_{0}}{2\left(\|x\|_{X}+\|y\|_{X}\right)}+\frac{y_{0}}{2\left(\|x\|_{X}+\|y\|_{X}\right)}\right\|_{X} \leq 1+\eta(1),
$$

$\log 0$

$$
\|x+y\|_{X} \leq 2(1+\eta(1)) \cdot\left(\|x\|_{X}+\|y\|_{X}\right)
$$

para todo $x, y \in X$.

Demonstraremos que $\|x\|_{X}=0$, implica $x=0$. De fato, suponha por contradição que $x \neq 0$. Observe que $\|n x\|_{X}=0$ para todo $n \in \mathbb{N}$, portanto, $n x \in B_{X}$ para todo $n \in \mathbb{N}$. Escolha $y \notin B_{X}$. Se $x_{n}=\min (n|x|,|y|)$, então $x_{n} \leq n|x|$ e pelo item 1. desta observação, temos que $x_{n} \in B_{X}$. Também $x_{n} \longrightarrow|y|$ q.s., isto é, $\left(x_{n}\right)_{n}$ converge em medida para $|y|$. Como $X$ é um espaço de funções $g$-convexos, temos que $B_{X}$ é fechada em $L_{0}$, assim $|y| \in B_{X}$, o que contradiz a escolha de $y$.

Com isto, nós concluímos que $\|\cdot\|_{X}$ é uma quase-norma.

Vamos demonstrar que $X$ é um espaço quase-Banach. Para isso demonstraremos que o espaço $(X, d)$ é completo, onde $d$ é a métrica definida em Definição 2.51, para facilitar os cálculos, escrevamos $\|x-y\|=d(x, y)=\|x-y\|^{p}$. De fato, seja $\left(x_{n}\right)_{n}$ uma sequência de Cauchy em $(X,\|\cdot\|)$. Vejamos que ela é convergente. De fato, por $\left(x_{n}\right)_{n}$ ser de Cauchy, encontramos uma subsequencia de $\left(x_{n}\right)_{n}$, que denotamos por $\left(y_{n}\right)_{n}$, tal que

$$
\sum_{k=1}^{\infty}\left\|y_{k+1}-y_{k}\right\|<\infty
$$


Definamos uma função $y$ por

$$
y(s)=\left|y_{1}(s)\right|+\sum_{k=1}^{\infty}\left|y_{k+1}(s)-y_{k}(s)\right|,
$$

a qual pode tomar o valor $\infty$ para alguns $s$.

Seja $\vartheta_{n}(s)=\left|y_{1}(s)\right|+\sum_{k=1}^{n}\left|y_{k+1}(s)-y_{k}(s)\right|$. Note que $\left(\vartheta_{n}\right)_{n}$ é uma sequência de $X$, com $\vartheta_{n} \rightarrow y$ q.s. (portanto, converge em medida), se definimos $\rho_{m}(s)=\inf _{n \geq m} \vartheta_{n}(s)$, então $\rho_{m} \leq \vartheta_{n}$, se $n \geq m$, e $\rho_{m} \rightarrow y$ q.s. quando $m \rightarrow \infty$, portanto, $\rho_{m} \in X$ e $\left\|\rho_{m}\right\|_{X} \leq\left\|\vartheta_{n}\right\|_{X}$ para $n \geq m$, assim $\left\|\rho_{m}\right\|_{X} \leq \liminf \left\|\vartheta_{n}\right\|_{X}$, como $B_{X}$ é fechada na topologia da medida, temos que $y \in X$ e $\|y\|_{X} \leq \liminf \left\|\vartheta_{n}\right\|_{X}$. Pela Observação 5.6, obtemos que o conjunto $E=\{s: y(s)=\infty\}$ tem medida zero, $\operatorname{logo}$ a serie $\sum_{k=1}^{\infty}\left|y_{k+1}(s)-y_{k}(s)\right|$ converge em $s \in S \backslash E$, assim para $s \in S \backslash E$ a serie $\sum_{k=1}^{\infty}\left(y_{k+1}(s)-y_{k}(s)\right)$ é convergente. Definamos uma função $x$ por

$$
x(s)= \begin{cases}y_{1}(s)+\sum_{k=1}^{\infty}\left(y_{k+1}(s)-y_{k}(s)\right) & \text { se } s \in S \backslash E, \\ 0 & \text { se } s \in E .\end{cases}
$$

Note que $|x| \leq y$, portanto, $x \in X$. Observe que

$$
\left|x(s)-y_{m}(s)\right| \leq \sum_{k=m}^{\infty}\left|y_{k+1}(s)-y_{k}(s)\right|, \text { se } s \in S \backslash E,
$$

$\log$,

$$
\left\|x-y_{m}\right\| \leq \sum_{k=m}^{\infty}\left\|y_{k+1}-y_{k}\right\| \rightarrow 0, \text { se } m \rightarrow \infty .
$$

Por outro lado,

$$
\left\|x-x_{n}\right\| \leq\left\|x-y_{m}\right\|+\left\|x_{n}-y_{m}\right\| \rightarrow 0,
$$

se $m \rightarrow \infty$ e se $n \rightarrow \infty$. Isto mostra que $(X,\|\cdot\|)$, isto é, $(X, d)$ é completo, portanto $\left(X,\|\cdot\|_{X}\right)$ é um espaço quase-Banach.

Definição 5.8 (Dual de Köthe) Seja X um espaço de Köthe. Definimos $X^{*}$, o Dual de Köthe de $X$, como o espaço de funções mensuráveis $x^{*}$ tais que

$$
\left\|x^{*}\right\|_{X^{*}}=\sup _{x \in B_{X}} \int_{S}\left|x x^{*}\right| d \mu
$$

existe. 
A função $\|\cdot\|_{X^{*}}$ define uma norma em $X^{*}$. Observe que $X^{*}$ pode ser visto como subespaço de $X^{\prime}$, identificando o elemento $x^{*} \in X^{*}$ pelo funcional linear $y(x)=\int_{S} x x^{*} d \mu$. De fato,

$$
\begin{aligned}
\|y\|_{X^{\prime}} & =\sup _{x \in B_{X}}|y(x)| \\
& =\sup _{x \in B_{X}}\left|\int_{S} x x^{*} d \mu\right| \\
& =\sup _{x \in B_{X}} \int_{S}\left|x x^{*}\right| d \mu \\
& =\left\|x^{*}\right\|_{X^{*}} .
\end{aligned}
$$

Exemplo 5.9 Se $1 \leq p<\infty$, temos que $L_{p}^{*}(\mu)=L_{q}(\mu)$, onde $\frac{1}{p}+\frac{1}{q}=1$.

Existem espacos $X$, tais que $X^{*} \neq X^{\prime}$, por exemplo, $L_{\infty}^{*}(\mu)=L_{1}(\mu)$.

Como é de esperar, $X^{*}$ é um espaço de Köthe sempre que $X$ seja um espaço de Köthe. A proposição abaixo prova esse fato.

Proposição 5.10 Se X é um espaço de Köthe, então $X^{*}$ é um espaço de Köthe.

Demonstração. Provemos que $B_{X^{*}}$ é fechada na topologia da convergência em medida. De fato, seja $\left(x_{n}^{*}\right)_{n}$ uma sequência de $B_{X^{*}}$ que converge em medida para $x^{*}$. Como $\mu$ é uma medida $\sigma$-finita, então encontramos uma subsequência de $\left(x_{n}^{*}\right)_{n}$ que converge q.s. para $x^{*}$, denotamos essa subsequência como $\left(x_{n}^{*}\right)_{n}$. Assumiremos que $\left|\left(x_{n}^{*}\right)_{n}\right|$ é uma sequência crescente que converge q.s. para $x^{*}$ (basta tomar a sequência $\left.y_{n}^{*}=\inf _{k \geq n}\left|x_{k}^{*}\right|\right)$. Seja $x \in B_{X}$ qualquer, observe que $\left|x_{n}^{*} \| x\right|$ é uma sequência crescente que converge q.s. para $\left|x^{*}\right||x|$, com

$$
\sup _{n} \int\left|x_{n}^{*}\left\|x \mid d \mu \leq \sup _{n}\right\| x_{n}^{*} \|_{X^{*}} \leq 1\right.
$$

Pelo Teorema 2.59 temos que $\left|x^{*} \| x\right| \in L_{1}$ e $\left|x_{n}^{*} \| x\right|$ converge para $\left|x^{*} \| x\right|$ em norma de $L_{1}$, assim

$$
\begin{aligned}
\int\left|x^{*} \| x\right| d \mu & =\lim _{n \rightarrow \infty} \int\left|x_{n}^{*}\right||x| d \mu \\
& \leq \sup _{n}\left\|x_{n}^{*}\right\|_{X^{*}} \\
& \leq 1,
\end{aligned}
$$

para todo $x \in B_{X}$, concluindo que 


$$
\sup _{x \in B_{X}} \int\left|x^{*} \| x\right| d \mu \leq 1
$$

e $\operatorname{assim} x^{*} \in B_{X^{*}}$.

Note que $B_{X^{*}}$ contém um elemento positivo, pois existe uma função positiva $h$ tal que $\|h x\|_{L_{1}} \leq$ $\|x\|_{X}$ para todo $x \in X$. Pela definição de $X^{*}$, temos que $h \in X^{*}, \operatorname{logo} h /\|h\|_{X^{*}} \in B_{X^{*}}$.

É imediato ver que se $|y| \leq|x| \operatorname{com} x \in X^{*}$, então $y \in X^{*}$ e $\|y\|_{X^{*}} \leq\|x\|_{X^{*}}$. Com isso acaba a prova desta proposição .

Vejamos que $X^{*}$ é isométrico a um subespaço normante (ver Definição 2.78 ) de $X^{\prime}$.

Proposição $5.11 X^{*}$ é um é isométrico a um subespaço normante de $X^{\prime}$.

Demonstração. Como $X$ é um espaço de Köthe, então existe $h$ positivo em $L_{0}$, tal que para todo $x \in X$, temos que $h x \in L_{1} \mathrm{e}\|x h\|_{L_{1}} \leq\|x\|_{X}$, isto é, $X \subseteq L_{1}(h d \mu)$.

Vejamos que $B_{X}$ é fechado em $L_{1}(h d \mu)$. De fato, seja $\left(x_{n}\right)_{n}$ sequência em $B_{X}$ tal que $x_{n} \longrightarrow x$ em norma de $L_{1}(h d \mu)$, isto é, $\left\|h x_{n}-h x\right\|_{L_{1}} \longrightarrow 0$, portanto, existe uma subsequência de $\left(x_{n}\right)_{n}$, que denotamos por $\left(x_{n}\right)_{n}$, tal que $\left(x_{n}\right)_{n}$ converge q.s. para $x$, assim pela Proposição 2.14 temos que $\left(x_{n}\right)_{n}$ converge em medida relativa a $x, \log x \in B_{X}$, pois $B_{X}$ é fechada na topologia da convergência em medida.

Seja $\left\|x_{0}\right\|_{X}>1$ fixo. Agora, como $B_{X}$ é fechado em $L_{1}(h d \mu)$, pelo teorema de separação de Hahn-Banach (Teorema 2.66), existe $x^{*} \in\left(L_{1}(h d \mu)\right)^{\prime}$ tal que

$$
\sup _{y \in B_{X}}\left|x^{*}(y)\right|=\sup _{y \in B_{X}} \operatorname{Re}\left(x^{*}(y)\right) \leq 1
$$

e $\left|x^{*}\left(x_{0}\right)\right|>1$. Porém, os funcionais $x^{*}$ em $\left(L_{1}(h d \mu)^{\prime}\right.$ são representados por

$$
x^{*}(y)=\int y h^{-1} u d \mu
$$

para alguma função $u \in L_{\infty}\left(h^{-1} d \mu\right)$, assim $h^{-1} u=x^{*} \in X^{*}$ e $x^{*} \in B_{X^{*}}$. Lembre-se que nós queremos demonstrar que

$$
\|x\|_{X}=\sup _{x^{*} \in B_{X^{*}}}\left|x^{*}(x)\right|
$$

para isso, é suficiente provar que para $\varepsilon>0$, existe $x^{*} \in B_{X^{*}}$ tal que

$$
\left|x^{*}(x)\right|>\|x\|_{X}-\varepsilon .
$$

Se tomamos $x_{0}=\frac{x}{\|x\|_{X}-\varepsilon}$, temos que $\left\|x_{0}\right\|_{X}>1$, logo existe $x^{*} \in B_{X^{*}}$ tal que $x^{*}\left(x_{0}\right)>1$, deste fato, deduzimos a desigualdade (5.1).

Proposição 5.12 Se $X_{0}$ e $X_{1}$ são espaços de Köthe, então

$$
\left(X_{0}^{*}\right)^{1-\theta} \cdot\left(X_{1}^{*}\right)^{\theta} \subset\left(X_{0}^{1-\theta} \cdot X_{1}^{\theta}\right)^{*}
$$


Demonstração. Sejam $x^{*} \in\left(X_{0}^{*}\right)^{1-\theta} \cdot\left(X_{1}^{*}\right)^{\theta}$ e $x_{0}^{*} \in X_{0}^{*}, x_{1}^{*} \in X_{1}^{*}$, tais que $\left|x^{*}\right|=\left|x_{0}^{*}\right|^{1-\theta}\left|x_{1}^{*}\right|^{\theta}$. Seja $x \in B_{X_{0}^{1-\theta} \cdot X_{1}^{\theta}}$ arbitrário. Então existem $x_{0} \in X_{0}$ e $x_{1} \in X_{1}$, tais que $|x|=\left|x_{0}\right|^{1-\theta}\left|x_{1}\right|^{\theta} \mathrm{e}$ $\left\|x_{0}\right\|_{X_{0}}^{1-\theta}\left\|x_{1}\right\|_{X_{1}}^{\theta} \leq 1$. Como $x_{0}^{*} \in X_{0}^{*}$ e $x_{1}^{*} \in X_{1}^{*}$, então $\left|x_{0}^{*}\left\|x_{0}|,| x_{1}^{*}\right\| x_{1}\right| \in L_{1}$, com

$$
\int\left|x_{0}^{*}\left\|x_{0} \mid d \mu \leq\right\| x_{0}^{*} \|_{X_{0}^{*}} \text { e } \int\right| x_{1}^{*}\left\|x_{1} \mid d \mu \leq\right\| x_{1}^{*} \|_{X_{1}^{*}}
$$

Agora pelos fatos de $\left(\left|x_{0}^{*} \| x_{0}\right|\right)^{1-\theta} \in L_{1 /(1-\theta)},\left(\left|x_{1}^{*} \| x_{1}\right|\right)^{\theta} \in L_{1 / \theta}$ e pela desigualdade de Hölder temos que

$$
\begin{aligned}
\int\left|x \| x^{*}\right| d \mu & =\int\left(\left|x_{1} \| x_{1}^{*}\right|\right)^{\theta} \cdot\left(\left|x_{0}^{*} \| x_{0}\right|\right)^{1-\theta} d \mu \\
& \leq\left(\int\left|x_{1}^{*} \| x_{1}\right| d \mu\right)^{\theta} \cdot\left(\int\left|x_{0}^{*} \| x_{0}\right| d \mu\right)^{1-\theta} \\
& \leq\left\|x_{1}^{*}\right\|_{X_{1}^{*}}^{\theta} \cdot\left\|x_{0}^{*}\right\|_{X_{0}^{*}}^{1-\theta} \cdot\left\|x_{1}\right\|_{X_{1}}^{\theta} \cdot\left\|x_{0}\right\|_{X_{0}}^{1-\theta} \\
& \leq\left\|x_{1}^{*}\right\|_{X_{1}^{*}}^{\theta} \cdot\left\|x_{0}^{*}\right\|_{X_{0}^{*}}^{1-\theta}
\end{aligned}
$$

para todo $x \in B_{X}$, portanto, $\left\|x^{*}\right\|_{\left(X_{0}^{1-\theta} \cdot X_{1}^{\theta}\right)^{*}} \leq\left\|x_{1}^{*}\right\|_{X_{1}^{*}}^{\theta} \cdot\left\|x_{0}^{*}\right\|_{X_{0}^{*}}^{1-\theta}$ para todo $x_{0}^{*} \in X_{0}^{*}$ e $x_{1}^{*} \in X_{1}^{*}$, tais que $\left|x^{*}\right|=\left|x_{0}^{*}\right|^{1-\theta}\left|x_{1}^{*}\right|^{\theta}$, assim

$$
\left\|x^{*}\right\|_{\left(X_{0}^{1-\theta} \cdot X_{1}^{\theta}\right)^{*}} \leq\left\|x^{*}\right\|_{\left(X_{0}^{*}\right)^{1-\theta} \cdot\left(X_{1}^{*}\right)^{\theta}} .
$$

Isto prova que

$$
\left(X_{0}^{*}\right)^{1-\theta} \cdot\left(X_{1}^{*}\right)^{\theta} \subset\left(X_{0}^{1-\theta} \cdot X_{1}^{\theta}\right)^{*}
$$

Lema 5.13 Seja X um espaço de Köthe e $0<\theta<1$. Então o espaço $\theta$-potência $X^{(\theta)}$ (ver Definição 2.41) satisfaz a seguintes propriedades:

- A bola fechada unitária em $X^{(\theta)}$ é fechada na topologia da convergência q.s.

- Existe um h positivo em $L_{0}$ tal que

$$
\|h x\|_{L_{1}} \leq\|x\|_{X^{(\theta)}}
$$

para todo $x \in X^{(\theta)}$. 
Demonstração. Para provar a primeira parte tomemos uma sequência $\left(z_{n}\right)_{n}$ em $B_{X^{(\theta)}}$ tal que converge q.s. para $z$. Imediatamente obtemos que $\left|z_{n}\right|^{1 / \theta} \longrightarrow|z|^{1 / \theta}$ q.s., isto implica que $\left|z_{n}\right|^{1 / \theta}$ converge em medida relativa para $|z|^{1 / \theta}$. Como $\left|z_{n}\right|^{1 / \theta}$ é uma sequência na bola unitária de $X$, nós concluimos pelo fato de $X$ ser um espaço de Köthe, que $z^{1 / \theta} \in X$ e assim $z \in X^{(\theta)}$.

Provaremos agora a segunda parte. De fato, seja $z \in X^{(\theta)}$. Como $X$ é um espaço de Köthe, então existe $\bar{h}>0$ tal que

$$
\|\bar{h} \bar{x}\|_{L_{1}} \leq\|\bar{x}\|_{X} \text { para todo } \bar{x} \in X
$$

em particular

$$
\left\|\bar{h}|z|^{1 / \theta}\right\|_{L_{1}} \leq\left\||z|^{1 / \theta}\right\|_{X}
$$

consequentemente,

$$
\left\|\bar{h}|z|^{1 / \theta}\right\|_{L_{1}} \leq\left\||z|^{1 / \theta}\right\|_{X}
$$

portanto,

$$
\left\|\bar{h}|z|^{1 / \theta}\right\|_{L_{1}}^{\theta} \leq\|z\|_{X^{(\theta)}}
$$

$\log 0$

$$
\left(\int \bar{h}|z|^{1 / \theta} d \mu\right)^{\theta} \leq\|z\|_{X^{(\theta)}}
$$

Como $S$ é $\sigma$-finita, então existe uma sequência de conjuntos mensuráveis disjuntos $\left(S_{n}\right)_{n}$ tal que $0<\mu\left(S_{k}\right)<\infty$ e $S=\bigcup_{k \in \mathbb{N}} S_{k}$, portanto,

$$
\left\|\bar{h} 1_{S_{k}}|z|^{1 / \theta}\right\|_{L_{1}} \leq\left\|1_{S_{k}}|z|^{1 / \theta}\right\|_{X}
$$

$\log 0$

$$
\left\|\bar{h} 1_{S_{k}}|z|^{1 / \theta}\right\|_{L_{1}}^{\theta} \leq\left\|1_{S_{k}}|z|^{1 / \theta}\right\|_{X}^{\theta}
$$

consequentemente,

$$
\left(\int_{S_{k}} \bar{h}|z|^{1 / \theta} d \mu\right)^{\theta} \leq\|z\|_{X^{(\theta)}}
$$

finalmente,

$$
\left(\mu\left(S_{k}\right) \cdot \frac{1}{\mu\left(S_{k}\right)} \int_{S_{k}} \bar{h}|z|^{1 / \theta} d \mu\right)^{\theta} \leq\|z\|_{X^{(\theta)}},
$$

e como $0<\theta<1$, a desigualdade de Jensen (ver Teorema 2.55) garante que

$$
\mu\left(S_{k}\right)^{\theta-1} \int_{S_{k}} \bar{h}^{\theta}|z| d \mu \leq\left(\mu\left(S_{k}\right) \cdot \frac{1}{\mu\left(S_{k}\right)} \int_{S_{k}} \bar{h}|z|^{1 / \theta} d \mu\right)^{\theta},
$$


escrevendo $c_{k}=\frac{1}{\mu\left(S_{k}\right)^{\theta-1}}$, obtemos que

$$
\left\|\bar{h} \cdot 1_{S_{k}} z\right\|_{L_{1}} \leq c_{k}\|z\|_{X^{(\theta)}}
$$

Definindo

$$
h(s)=\frac{1_{S_{k}} \cdot \bar{h}(s)}{2^{k} \cdot c_{k}}, \text { se } s \in S_{k},
$$

temos que

$$
\begin{aligned}
\|h z\|_{L_{1}} & =\sum_{k=1}^{\infty} \frac{1}{2^{k} \cdot c_{k}}\left\|\bar{h} 1_{S_{k}} z\right\|_{L_{1}} \\
& \leq \sum_{k=1}^{\infty} \frac{1}{2^{k} \cdot c_{k}} \cdot c_{k}\|z\|_{X^{(\theta)}} \\
& =\|z\|_{X^{(\theta)}}
\end{aligned}
$$

Proposição 5.14 Se $\left(X_{0}, X_{1}\right)$ é um par interpolados de espaços de Köthe, então $X_{\theta}=X_{0}^{1-\theta} X_{1}^{\theta}$ é um espaço de Köthe para $0<\theta<1$.

Demonstração. Vamos aprovar o teorema por partes:

Passo 1. Primeiro, encontremos $h \in L_{0}$ positivo tal que $\|h x\|_{L_{1}} \leq\|x\|_{X_{\theta}}$ para todo $x \in X_{\theta}$. Pela hipótese de $X_{0}$ e $X_{1}$ serem espaços de Köthe, existem $h_{0}, h_{1} \in L_{0}$ positivos tais que $\left\|h_{0} x_{0}\right\|_{L_{1}} \leq$ $\left\|x_{0}\right\|_{X_{0}}$ para todo $x_{0} \in X_{0} \mathrm{e}\left\|h_{1} x_{1}\right\|_{L_{1}} \leq\|x\|_{X_{1}}$ para todo $x_{1} \in X_{1}$. Então, para todo $x_{0}$ e $x_{1}$ tais que $|x|=\left|x_{0}\right|^{1-\theta}\left|x_{1}\right|^{\theta}$ e pela desigualdade de Hölder, temos que

$$
\begin{aligned}
\left\|x_{0}\right\|_{X_{0}}^{1-\theta}\left\|x_{1}\right\|_{X_{1}}^{\theta} & \geq\left\|h_{0} x_{0}\right\|_{L_{1}}^{1-\theta}\left\|h_{1} x_{1}\right\|_{L_{1}}^{\theta} \\
& =\left(\int\left(\left|h_{0} x_{0}\right|^{1-\theta}\right)^{1 /(1-\theta)} d \mu\right)^{1-\theta}\left(\int\left(\left|h_{1} x_{1}\right|^{\theta}\right)^{1 / \theta} d \mu\right)^{\theta} \\
& \geq \int\left|h_{0} x_{0}\right|^{1-\theta}\left|h_{1} x_{1}\right|^{\theta} d \mu \\
& =\left\|h_{0}^{1-\theta} h_{1}^{\theta} x\right\|_{L_{1}} .
\end{aligned}
$$

Se $h=h_{0}^{1-\theta} h_{1}^{\theta}$, nós concluimos que $\|h x\|_{L_{1}} \leq\|x\|_{X_{\theta}}$ para todo $x \in X_{\theta}$. 
Passo 2. A bola unitária $B_{X_{\theta}}$ contém um elemento positivo. De fato, como $X_{0}$ e $X_{1}$ são espaços de Köthe, então existem elementos positivos $x_{0}$ e $x_{1}$ em $B_{X_{0}}$ e $B_{X_{1}}$, respectivamente. Definindo $|x|=\left|x_{0}\right|^{1-\theta}\left|x_{1}\right|^{\theta}$, temos que $|x|$ é um elemento positivo de $B_{X_{\theta}}$.

Passo 3. Afirmamos que

$$
\text { se }|y| \leq|x| \text {, com } x \in X_{\theta} \text {, então } y \in X_{\theta} \text { e }\|y\|_{X_{\theta}} \leq\|x\|_{X_{\theta}} \text {. }
$$

De fato, sejam $x_{0}$ e $x_{1}$ tais que $|x|=\left|x_{0}\right|^{1-\theta}\left|x_{1}\right|^{\theta}$. Agora

$$
\left(|y|\left|x_{1}\right|^{-\theta}\right)\left|x_{1}\right|^{\theta} \leq\left|x_{0}\right|^{1-\theta}\left|x_{1}\right|^{\theta}
$$

o que implica $\left|y_{0}\right|=\left(\left|y \| x_{1}\right|^{-\theta}\right)^{1 /(1-\theta)} \leq\left|x_{0}\right|$, assim $y_{0} \in X_{0}$ e $\left\|y_{0}\right\|_{X_{0}} \leq\left\|x_{0}\right\|_{X_{0}}$. Se escrevemos $\left|y_{1}\right|=\left|x_{1}\right|$, obtemos que $|y|=\left|y_{0}\right|^{1-\theta}\left|y_{1}\right|^{\theta}$, isto é, $y \in X_{\theta}$. É imediato ver que

$$
\|y\|_{X_{\theta}} \leq\left\|y_{0}\right\|_{X_{0}}^{1-\theta}\left\|y_{1}\right\|_{X_{1}}^{\theta} \leq\left\|x_{0}\right\|_{X_{0}}^{1-\theta}\left\|x_{1}\right\|_{X_{1}}^{\theta},
$$

portanto, $\|y\|_{X_{\theta}} \leq\left\|x_{0}\right\|_{X_{0}}^{1-\theta}\left\|x_{1}\right\|_{X_{1}}^{\theta}$ para todo $x_{0}$ e todo $x_{1}$ tais que $|x|=\left|x_{0}\right|^{1-\theta}\left|x_{1}\right|^{\theta}, \operatorname{logo}$ $\|y\|_{X_{\theta}} \leq\|x\|_{X_{\theta}}$.

Passo 4. Provaremos que $B_{X_{\theta}}$ é fechada em $L_{0}$. Seja $\left(x_{n}\right)_{n}$ uma sequência em $B_{X_{\theta}}$ que converge em medida para $x$. Vejamos que $x \in B_{X_{\theta}}$. De fato, como $\left(x_{n}\right)_{n}$ converge em medida para $x$, então , existe uma subsequência de $\left(x_{n}\right)_{n}$ que converge q.s. para $x$, que denotamos por $\left(x_{n}\right)_{n}$. Existem sequências $\left(v_{n}\right)_{n}$ e $\left(w_{n}\right)_{n}$ não negativas em $X_{0}$ e $X_{1}$, respectivamente, tais que $\left\|v_{n}\right\|_{X_{0}}^{1-\theta}=1 \mathrm{e}$ $\left\|w_{n}\right\|_{X_{1}}^{\theta} \leq 1+\frac{1}{2^{n}} \operatorname{com}\left|x_{n}\right|=v_{n}^{1-\theta} w_{n}^{\theta}$. Seja $X_{1}^{(\theta)}$ o espaço $\theta$-potência de espaço $X_{1}$ (ver Subseção 2.3.1). Lembre-se que o espaço $X_{1}^{(\theta)}$ é definido por

$$
X_{1}^{(\theta)}=\left\{x \in L_{0}:|x|^{1 / \theta} \in X_{1}\right\}
$$

e normado por

$$
\|x\|_{X_{1}^{(\theta)}}=\left\||x|^{1 / \theta}\right\|_{X_{1}}^{\theta}
$$

Se denotamos $\bar{w}_{n}=w_{n}^{\theta}$, temos que $\bar{w}_{n} \in X_{1}^{(\theta)}$ e $\left\|\bar{w}_{n}\right\|_{X_{1}^{(\theta)}}=\left\|w_{n}\right\|_{X_{1}}^{\theta}$, portanto $\left\|\bar{w}_{n}\right\|_{X_{1}^{(\theta)}} \leq 1+\frac{1}{2^{n}}$. Doravante, dividimos a prova do Passo 4. para facilitar sua compreensão.

Passo 4.A. Sejam $\left(v_{n}\right)_{n}$ e $\left(w_{n}\right)_{n}$ do Passo 4. Demonstraremos que existem subsequências $\left(\bar{w}_{(n, n)}\right)_{n}$ e $\left(\bar{v}_{(n, n)}\right)_{n}$, de $\left(\bar{w}_{n}\right)_{n}$ e $\left(\bar{v}_{n}\right)_{n}$, respectivamente, com $|x|=\bar{w}_{(n, n)} \bar{v}_{(n, n)}$ e elementos $\bar{w} \geq 0$ e $\bar{v} \geq 0$ em $B_{X_{1}^{(\theta)}}$ e $B_{X_{0}^{(1-\theta)}}$, respectivamente, tais que para toda subsequência $\left(g_{n_{k}}\right)_{k}$ de $\left(\bar{w}_{(n, n)}\right)_{n}$

$$
\frac{1}{n} \sum_{k=1}^{n} g_{n_{k}} \stackrel{\text { q.s. }}{\longrightarrow} \bar{w}
$$


e toda para toda subsequência $\left(f_{n_{k}}\right)_{k}$ de $\left(\bar{v}_{(n, n)}\right)_{n}$

$$
\frac{1}{n} \sum_{k=1}^{n} f_{n_{k}} \stackrel{\text { q.s. }}{\longrightarrow} \bar{v} .
$$

De fato, o espaço $S$ pode-se escrever como $S=\bigcup_{k \in \mathbb{N}} S_{k}$, onde $S_{k} \subseteq S_{k+1}$ e $\mu\left(S_{k}\right)<\infty$ para todo $k \in \mathbb{N}$ (lembre-se que $\mu$ é $\sigma$-finita). Pelo Lema 5.13, item 2 e pelo fato de ser $\left(\bar{w}_{n}\right)_{n}$ uma sequência limitada em $X_{1}^{(\theta)}$, obtemos que $\left(h \bar{w}_{n}\right)_{n}$ é uma sequência limitada em $L_{1}(S, \mu)$, portanto, limitada em $L_{1}\left(S_{1}, \mu\right)$, assim, pelo teorema de Komlós (ver Teorema 2.57), encontramos uma subsêquencia $\left(\bar{w}_{(1, n)}\right)_{n}$ de $\left(\bar{w}_{n}\right)_{n}$ tal que para qualquer subsequência $\left(g_{n}\right)_{n}$ de $\left(\bar{w}_{(1, n)}\right)_{n}$, a sequência $\left(h g_{n}\right)_{n}$ é Cesàro-convergente para algum $\bar{g}_{1} \in L_{1}\left(S_{1}, \mu\right)$ em $S_{1}$, isto é,

$$
\frac{1}{n} \sum_{k=1}^{n} h g_{k} \stackrel{q . s .}{\longrightarrow} \bar{g}_{1},
$$

se $n \rightarrow \infty$.

Similarmente $\left(h \bar{w}_{(1, n)}\right)_{n}$ é limitada em $L_{1}\left(S_{2}, \mu\right)$, usando novamente o teorema de Komlós, encontramos uma subsêquencia $\left(\bar{w}_{(2, n)}\right)_{n}$ de $\left(\bar{w}_{(1, n)}\right)_{n}$ tal que para qualquer subsequência $\left(g_{n}\right)_{n}$ de $\left(\bar{w}_{(2, n)}\right)_{n}$, a sequência $\left(h g_{n}\right)_{n}$ é Cesàro-convergente para algum $\bar{g}_{2} \in L_{1}\left(S_{2}, \mu\right)$ em $S_{2}$,isto é,

$$
\frac{1}{n} \sum_{k=1}^{n} h g_{k} \stackrel{q . s .}{\longrightarrow} \bar{g}_{2},
$$

se $n \rightarrow \infty$.

Observe que $\bar{g}_{2}=\bar{g}_{1}$ em $S_{1}$, pois $S_{1} \subseteq S_{2}$. Usando indução, encontramos para $j \in \mathbb{N}$ fixo, uma subsequência $\left(\bar{w}_{(j, n)}\right)_{n}$ de $\left(\bar{w}_{(j-1, n)}\right)_{n}$, portanto de $\left(\bar{w}_{n}\right)_{n}$, e $\bar{g}_{j} \in L_{1}\left(S_{j}, \mu\right)$, com $\bar{g}_{j}=\bar{g}_{j-1}$ em $S_{j-1}$, tal que para toda subsequência $\left(g_{n}\right)_{n}$ de $\left(\bar{w}_{(j, n)}\right)_{n}$ a sequência $\left(h g_{n}\right)_{n}$ é Cesàro-convergente para $\bar{g}_{j}$ em $S_{1}$, isto é,

$$
\frac{1}{n} \sum_{k=1}^{n} h g_{k} \stackrel{q . s .}{\longrightarrow} \bar{g}_{j},
$$

se $n \rightarrow \infty$.

Assumamos sem perda de generalidade que $\bar{w}_{(j+1, n)}=\bar{w}_{(j, n)}$ para todo $n \leq j+1$. Definamos uma função $\bar{g}$ em $S$ como $\bar{g}(s):=\bar{g}_{j}(s)$ se $s \in S_{j}$. Ela está bem definida, pois se $s \in S_{j} \cap S_{j+1}$, então $\bar{g}_{j}(s)=\bar{g}_{j+1}(s)$. Também, $\bar{g}$ assim definida está em $L_{0}$. Agora tomando a subsequência diagonal $\left(\bar{w}_{(n, n)}\right)_{n}$, obtemos uma subsequência de $\left(\bar{w}_{(j, n)}\right)_{n}$ para $j \in \mathbb{N}$ tal que para qualquer subsequência $\left(g_{n}\right)_{n}$ de $\left(\bar{w}_{(n, n)}\right)_{n}$, obtemos que

$$
\frac{1}{n} \sum_{k=1}^{n} h g_{k} \stackrel{q . s .}{\longrightarrow} \bar{g}
$$

em $S$. Demonstraremos agora que $\bar{g} h^{-1} \in X_{1}^{(\theta)}$. De fato, seja $G_{n}=\frac{1}{n} \sum_{k=1}^{n} g_{k}$ 


$$
\begin{aligned}
\left\|G_{n}\right\|_{X_{1}^{(\theta)}} & \leq \frac{1}{n} \sum_{k=1}^{n}\left\|g_{k}\right\|_{X_{1}^{(\theta)}} \\
& \leq \frac{1}{n} \sum_{k=1}^{n}\left(1+\frac{1}{2^{k}}\right) \\
& \leq 1+\frac{1}{n} .
\end{aligned}
$$

Fixemos $j \in \mathbb{N}$. Então $\left\|G_{n}\right\|_{X_{1}^{(\theta)}} \leq 1+\frac{1}{j}$ sempre que $n \geq j$. Se definimos a subsequência $\bar{G}_{n}=G_{j+n}$, de $\left(G_{n}\right)_{n}$, temos que $\left\|\bar{G}_{n}\right\|_{X_{1}^{(\theta)}} \leq 1+\frac{1}{j}$ para todo $n \in \mathbb{N}$. Também obtemos que $\bar{G}_{n} \stackrel{q . s .}{\longrightarrow} h^{-1} \bar{g}$, e por $B_{X_{1}^{(\theta)}}$ ser fechada na topologia da convergência q.s. (ver Lema 5.13, item 1), concluimos que $h^{-1} \bar{g} \in X_{1}^{(\theta)} \mathrm{e}$

$$
\left\|h^{-1} \bar{g}\right\|_{X_{1}^{(\theta)}} \leq 1+\frac{1}{j}
$$

para $j$ natural arbitrário, logo $\left\|h^{-1} \bar{g}\right\|_{X_{1}^{(\theta)}} \leq 1$. Denotemos por $\bar{w}=h^{-1} \bar{g} \geq 0$. Resumindo, existe uma subsequência $\left(\bar{w}_{(n, n)}\right)_{n}$ de $\left(\bar{w}_{n}\right)_{n}$ e um elemento $\bar{w} \geq 0$ em $B_{X_{1}^{(\theta)}}$ tal que para toda subsequência $\left(g_{n_{k}}\right)_{k}$ de $\left(\bar{w}_{(n, n)}\right)_{n}$, temos que

$$
\frac{1}{n} \sum_{k=1}^{n} g_{n_{k}} \stackrel{q . s .}{\longrightarrow} \bar{w} .
$$

Usando o mesmo argumento, encontramos uma subsequência $\left(\bar{v}_{(n, n)}\right)_{n}$ de $\left(\bar{v}_{n}\right)_{n}$ e um elemento $\bar{v} \geq 0$ em $B_{X_{0}^{(1-\theta)}}$ tal que para toda subsequência $\left(f_{n_{k}}\right)_{k}$ de $\left(\bar{v}_{(n, n)}\right)_{n}$

$$
\frac{1}{n} \sum_{k=1}^{n} f_{n_{k}} \stackrel{\text { q.s. }}{\longrightarrow} \bar{v}
$$

$\operatorname{com}|x|=\bar{w}_{(n, n)} \bar{v}_{(n, n)}$.

Passo 4.B. Vejamos que $x \in B_{X_{\theta}}$. De fato, Como $|x|=\bar{w}_{(n, n)} \bar{v}_{(n, n)}$,

$$
\frac{1}{n} \sum_{k=1}^{n} \bar{w}_{(k, k)} \stackrel{q . s .}{\longrightarrow} \bar{w}
$$

e

$$
\frac{1}{n} \sum_{k=1}^{n} \bar{v}_{(k, k)} \stackrel{q . s .}{\longrightarrow} \bar{v}
$$


a Proposição 2.77 implica que $|x| \leq \bar{w} \bar{v}$. Afirmamos que existe $\bar{w} \in X_{1}^{(\theta)}$ tal que $|x|=\bar{w} \bar{v}$. De fato, pela desigualdade $\frac{|x|}{\bar{v}} \leq \bar{w}$ e pelo fato de ser $X_{1}^{(\theta)}$ um espaço de Banach reticulado, temos que $\frac{|x|}{\bar{v}} \in X_{1}^{(\theta)} \mathrm{e}$

$$
\left\|\frac{|x|}{\bar{v}}\right\|_{X_{1}^{(1 / \theta)}} \leq\|\bar{w}\|_{X_{1}^{(1 / \theta)}} \leq 1
$$

Nós demonstramos que existem $\bar{w} \in X_{1}^{(\theta)}$ e $\bar{v} \in X_{1}^{(1-\theta)}$ tais que $|x|=\bar{w} \bar{v}$, com $\bar{w} \in B_{X_{1}^{(\theta)}} \mathrm{e}$ $\bar{v} \in B_{X_{0}^{(1-\theta)}}$. Se escrevemos $\bar{w}^{1 / \theta}=u_{1} \mathrm{e} \bar{v}^{1 /(1-\theta)}=u_{0}$, temos que $u_{i} \in B_{X_{i}}$ para $i=0,1 \mathrm{e}$

$$
|x|=u_{0}^{1-\theta} u_{1}^{\theta}
$$

daquí $x \in B_{X_{\theta}}$.

Proposição 5.15 Se X é um espaço de Köthe p-convexo, então o espaço $X^{(p)}$, que denotamos por $X^{p}$, é um espaço de Köthe.

Demonstração. Vamos dividir a prova em partes:

Passo 1. $X^{p}$ é um espaço normado com $\|\cdot\|_{X^{p}}$. De fato,

$$
0=\|x\|_{X^{p}}=\left\||x|^{\frac{1}{p}}\right\|_{X}^{p} \Leftrightarrow|x|=0 \Leftrightarrow x=0 .
$$

Para $\lambda$ escalar,temos que

$$
\|\lambda x\|_{X^{p}}=\left\||\lambda x|^{\frac{1}{p}}\right\|_{X}^{p}=|\lambda|\left\||x|^{\frac{1}{p}}\right\|_{X}^{p}=|\lambda|\|x\|_{X^{p}} .
$$

Vamos provar a desigualdade triangular. De fato, como $X$ é um espaço $p$-convexo, segue-se

$$
\left\|\left(\left|x_{0}\right|^{p}+\left|y_{0}\right|^{p}\right)^{1 / p}\right\|_{X} \leq\left(\left\|x_{0}\right\|_{X}^{p}+\left\|y_{0}\right\|_{X}^{p}\right)^{1 / p}
$$

para todo $x_{0}, y_{0} \in X$. Se $x, y \in X^{p}$, então $|x|^{1 / p},|y|^{1 / p} \in X$, substituindo $x_{0}, y_{0}$ respectivamente por $|x|^{1 / p},|y|^{1 / p}$ em (5.2), obtemos que

$$
\left\|(|x|+|y|)^{1 / p}\right\|_{X} \leq\left(\left\||x|^{1 / p}\right\|_{X}^{p}+\left\||y|^{1 / p}\right\|_{X}^{p}\right)^{1 / p}
$$

Por outro lado, $|x+y|^{1 / p} \leq(|x|+|y|)^{1 / p}$ e por $X$ ser um espaço de Köthe, deduzimos $\left\||x+y|^{1 / p}\right\|_{X} \leq$ $\left\|(|x|+|y|)^{1 / p}\right\|_{X}$, desta desigualdade e (5.3), segue-se que 


$$
\left\||x+y|^{1 / p}\right\|_{X} \leq\left(\left\||x|^{1 / p}\right\|_{X}^{p}+\left\||y|^{1 / p}\right\|_{X}^{p}\right)^{1 / p}
$$

e assim

$$
\|x+y\|_{X^{p}} \leq\|x\|_{X^{p}}+\|y\|_{X^{p}} .
$$

Passo 2. A bola unitária $B_{X^{p}}$ é fechada em $L_{0}$. De fato, seja $\left(x_{n}\right)_{n}$ uma sequência em $B_{X^{p}}$ que converge en medida para $x$. Note que $\left|x_{n}\right|^{1 / p}$ converge em medida para $|x|^{1 / p}$. Como $\left(\left|x_{n}\right|^{1 / p}\right)_{n}$ é uma sequência em $B_{X}$, segue-se que $|x|^{1 / p} \in B_{X}$, assim $x \in B_{X^{p}}$.

Passo 3. Demonstraremos que existe $h$ positivo tal que

$$
\|h x\|_{L_{1}} \leq\|x\|_{X^{p}}
$$

para todo $x \in X^{p}$. De fato, por $X$ ser um espaço de Köthe, existe $\bar{h}$ positiva tal que

$$
\|\bar{h} y\|_{L_{1}} \leq\|y\|_{X}
$$

para todo $y \in X$. Suponha $x \in X^{p}$ tal que $\|x\|_{X^{p}}=1$. Então $|x|^{1 / p} \in X$ e segue-se que

$$
\left\|\bar{h}|x|^{1 / p}\right\|_{L_{1}} \leq 1
$$

isto implica que existe um conjunto $A, \operatorname{com} \mu(A)=0$, tal que

$$
\bar{h}(s)|x(s)|^{1 / p} \leq 1
$$

para todo $s \in S / A$, portanto

$$
\bar{h}^{p}(s)|x(s)| \leq 1,
$$

se $s \in S / A$. Agora, por $\mu$ ser uma medida $\sigma$-finita, escrevemos $S=\cup_{k \in \mathbb{N}} S_{k}$, onde $0<\mu\left(S_{k}\right)<\infty$ e $\left(S_{k}\right)_{k}$ é uma sequência de conjuntos disjuntos. Note que

$$
\left\|\bar{h}^{p} x 1_{S_{k}}\right\|_{L_{1}} \leq \mu\left(S_{k}\right)
$$

Definindo

$$
h(s)=\frac{\bar{h}^{p}(s)}{2^{k} \cdot \mu\left(S_{k}\right)}, \text { se } s \in S_{k},
$$

obtemos que

$$
\begin{aligned}
\|x h\|_{L_{1}} & =\sum_{k=1}^{\infty} \int_{S_{k}}|x(s)| h(s) d \mu \\
& =\sum_{k=1}^{\infty}\left\|x \cdot \frac{\bar{h}^{p} \cdot 1_{S_{k}}}{2^{k} \cdot \mu\left(S_{k}\right)}\right\|_{L_{1}} \\
& \leq 1 .
\end{aligned}
$$


Agora para $x \in X^{p}, \operatorname{com} x \neq 0$, temos que

$$
\left\|\frac{x}{\|x\|_{X^{p}}} \cdot h\right\|_{L_{1}} \leq 1
$$

$\operatorname{assim}\|x h\|_{L_{1}} \leq\|x\|_{X^{p}}$.

Passo 4. Verifiquemos a condição da definiçao de espaço de Köthe. De fato, suponhamos $|x(s)| \leq|y(s)|$ para quase todo $s \in S$ com $x$ mensurável e $y \in X^{p}$. Observe que $|x(s)|^{1 / p} \leq|y(s)|^{1 / p}$ para quase todo $s \in S$, onde $|y|^{1 / p} \in X$, pelo fato de $X$ ser um espaço de Köthe, temos que $|x|^{1 / p} \in X \mathrm{e}$

$$
\left\||x|^{1 / p}\right\|_{X}^{p} \leq\left\||y|^{1 / p}\right\|_{X}^{p}
$$

isto mostra $x \in X^{p} \mathrm{e}\|x\|_{X^{p}} \leq\|y\|_{X^{p}}$. 
$\Gamma_{\text {Capítulo }}$

\section{O indicador de um espaço de funções} g-convexo

Neste capítulo introduzimos o conceito de indicador de um espaço de funções $g$-convexo e provamos algumas das suas propriedades.

Definição 6.1 (Semi-ideal) Um subconjunto $\mathscr{T}$ do cone $L_{1}^{+}$é chamado de semi-ideal, se $\mathscr{T} e ́$ um cone e verifica que

$$
\text { se } 0 \leq f \leq g \text {, com } g \in \mathscr{T} \text {, implica que } f \in \mathscr{T} \text {. }
$$

Além disso, se $\mathscr{T}$ contém um elemento estritamente positivo, então $\mathscr{T}$ é chamado semi-ideal estrito.

Definição 6.2 (Semi-ideal associado a um espaço de funções $g$-convexos) Seja $X$ um espaço de funções g-convexos. Para cada $x \in X$, consideremos o conjunto $A=\{s \in S:|x(s)|>1\}$.

Nós definimos $\mathscr{T}_{X}$ como o semi-ideal de todos os $f \in L_{1}^{+}$tais que

$$
\sup _{x \in B_{X}} \int_{A} f \log |x| d \mu<\infty
$$

- e existe $x \in B_{X}$ tal que

$$
\int_{A} f|\log | x \| d \mu<\infty .
$$

Denotamos simplesmente por $\int f \log _{+}|x| d \mu$ a integral $\int_{A} f \log |x| d \mu$, onde $A=\{s \in S$ : $|x(s)|>1\}$. 
Definição 6.3 (Indicador de um espaço de funções $g$-convexo) Para $f \in \mathscr{T}_{X}$ definimos $o$ indicador de $X$, por

$$
\Phi_{X}(f)=\sup _{x \in B_{X}} \int_{S} f \log |x| d \mu
$$

Observe que a definição de $\mathscr{T}_{X}$ implica que $-1 \Phi_{X}(f) \in \mathbb{R}$ para todo $f \in \mathscr{T}_{X}$.

Denotamos como $L \log L$ o subconjunto de $L_{1}$ tal que $f(1+|\log | f||) \in L_{1}$.

\subsection{Propriedades dos indicadores}

Proposição 6.4 Se $1 \leq p<\infty$, então $\mathscr{T}_{L_{p}}=(L \log L)_{+} e$

$$
\Phi_{L_{p}}(f)=\frac{1}{p} \int_{S} f \log \frac{f}{\|f\|_{L_{1}}} d \mu .
$$

Demonstração. Suponhamos $f \in(L \log L)_{+}$, isto é, $f(1+|\log | f||) \in L_{1}$ e $f>0$. Sem perda de generalidade podemos supor $\|f\|_{L_{1}}=1$. Seja $\|x\|_{L_{p}} \leq 1$. Então

$$
\begin{aligned}
\int f \log _{+}|x| d \mu & =\frac{1}{p} \int_{A} f \log |x|^{p} d \mu \\
& =\frac{1}{p} \int_{A} f \log f d \mu+\frac{1}{p} \int_{A} f \log \frac{|x|^{p}}{f} d \mu \\
& \leq \frac{1}{p} \int_{A} f \log |f| d \mu+\frac{1}{p} \int_{A}|x|^{p} d \mu \\
& \leq \frac{1}{p} \int_{A} f|\log | f\left\|d \mu+\frac{1}{p}\right\| x \|_{L_{p}}^{p} \\
& \leq \frac{1}{p} \int_{A} f|\log | f \| d \mu+\frac{1}{p}
\end{aligned}
$$

$\operatorname{logo} \int f \log _{+}|x| d \mu<\infty$, portanto, (6.1) é verdadeira. Para (6.2) basta tomar $x=f^{1 / p}$. Nós mostramos que $(L \log L)_{+} \subset \mathscr{T}_{L_{p}}$.

Reciprocamente, seja $f \in \mathscr{T}_{L_{p}}$ e suponha sem perda de generalidade que $\|f\|_{L_{1}}=1$. Agora por (6.2), existe $x \in B_{L_{P}}$ tal que $f \log |x|$ é integrável. Pela desigualdade de Jensen (ver Teorema 2.55), temos que

$$
\int f \log \frac{|x|^{p}}{f} d \mu \leq \log \int|x|^{p} d \mu=\log \|x\|_{L_{p}}^{p} d \mu \leq 0
$$




\subsection{Propriedades dos indicadores}

e desta desigualdade concluimos

$$
\int f \log |x|^{p} d \mu \leq \int f \log f d \mu,
$$

isto é

$$
\int f \log |x| d \mu \leq \int f \log f^{1 / p} d \mu
$$

A integral da direita existe, pois (6.1) vale e em particular vale para $f^{1 / p} \in B_{L_{p}}$. Logo

$$
\Phi_{L_{p}}(f)=\frac{1}{p} \int_{S} f \log f d \mu .
$$

Observe que $\Phi_{X}$ é uma função homogênea, portanto, a igualdade vale para todo $f \neq 0$.

Observe que denotando $\Lambda=\Phi_{L_{1}}$, temos que $\Phi_{L_{p}}=\frac{1}{p} \Lambda$.

Teorema 6.5 (Fatoração de Lozanovskii) Seja $X$ um espaço de Köthe e $f \in L_{1}(\mu)$. Então existe um único par $\left(x, x^{*}\right)$ em $X \times X^{*}$, com $x \geq 0,\|x\|_{X}=1,\left\|x^{*}\right\|_{X^{*}}=\|f\|_{L_{1}} e \operatorname{supp}(x)=$ $\operatorname{supp}\left(x^{*}\right)=\operatorname{supp}(f)$ tal que $f=x \cdot x^{*}$.

A prova deste teorema pode ser vista na referência [25]. Por simplicidade, vamos dar uma prova da fatoração de Lozanovskii para espaços de Banach de sequências (Ver seção 2.3.3).

Teorema 6.6 (Fatoração de Lozavovskii para E.B.S) Sejam $X$ um espaço de Banach de sequências e $u \in l_{1}, u \geq 0$ com $\|u\|_{l_{1}}=1$. Então existem $x \in B_{X}$ e $x^{*} \in B_{X^{*}}, x \geq 0, x^{*} \geq 0$ tais que $u=x \cdot x^{*}$. Os elementos $x^{*}$ e $x$ são únicos se assumirmos que $x_{n}=x_{n}^{*}=0$ sempre que $u_{n}=0$.

Demonstração. Assumamos primeiro que $u \in c_{00}$ fixo, onde $c_{00}$ denota o espaço das sequências de suporte finito. Escolhamos $x \in B_{X}$, com $x \geq 0$, tal que, maximize

$$
\sum_{n=1}^{\infty} u_{n} \log \left|x_{n}\right|
$$

escrevendo por conveniência $\log 0:=0$. Note que tal $x$ existe, pois a função

$$
\left(x_{1}, x_{2}, \ldots, x_{n}\right) \mapsto \sum_{n=1}^{\infty} u_{n} \log \left|x_{n}\right|
$$

definida em $\mathbb{R}^{n}$ atinge um máximo em $B_{\mathbb{R}^{n}}$ pelo fato de ser contínua. Se $\xi \in B_{X} \operatorname{com} \xi \geq 0$, então 


\subsection{Propriedades dos indicadores}

$$
\sum_{n=1}^{\infty} u_{n} \log \left(x_{n}+t\left(\xi_{n}-x_{n}\right)\right) \leq \sum_{n=1}^{\infty} u_{n} \log x_{n}
$$

para todo $0 \leq t \leq 1$, pois $x+t(\xi-x) \in B_{X}$ devido a que $B_{X}$ é convexa e pela forma como nós escolhemos $x$. Observe que se $u_{n}>0$, então $x_{n}>0$. Derivando a expressão

$$
\sum_{n=1}^{\infty} u_{n} \log \left(x_{n}+t\left(\xi_{n}-x_{n}\right)\right)
$$

em relação a $t$ obtemos

$$
\sum_{u_{n} \neq 0} u_{n} \frac{\xi_{n}-x_{n}}{x_{n}+t\left(\xi_{n}-x_{n}\right)}
$$

A expressão (6.4) tem um máximo em $t=0$, portanto a derivada nesse ponto é não positiva, isto é,

$$
\sum_{u_{n} \neq 0} u_{n} \frac{\xi_{n}-x_{n}}{x_{n}} \leq 0 .
$$

Definamos $x_{n}^{*}=\frac{u_{n}}{x_{n}}$ se $x_{n} \neq 0$ e zero nos outros casos. Da desigualdade (6.5), temos que

$$
\sum_{n=1}^{\infty} \xi_{n} x^{*} \leq \sum_{n=1}^{\infty} u_{n} \leq 1,
$$

assim $\left\|x^{*}\right\|_{X^{*}} \leq 1$. Com isso, provamos a parte da existência do teorema.

Agora provaremos a unicidade do teorema. De fato, suponha que existam $y \in B_{X}$ e $y^{*} \in B_{X^{*}}$ tais que $y \geq 0, y^{*} \geq 0$ e $u=y \cdot y^{*}$. Pela convexidade das bolas obtemos que

$$
\frac{x+y}{2} \in B_{X} \quad \text { e } \quad \frac{x^{*}+y^{*}}{2} \in B_{X^{*}}
$$

$\log 0$

$$
\sum_{n=1}^{\infty} \frac{1}{4}\left(x_{n}+y_{n}\right)\left(x_{n}^{*}+y_{n}^{*}\right) \leq\left\|\frac{x_{n}^{*}+y_{n}^{*}}{2}\right\|_{X^{*}} \leq 1 .
$$

Usando a desigualdade acima, os fatos $u=x \cdot x^{*}=y \cdot y^{*}$ e $\sum_{k=1}^{n} u_{n}=1$, provamos que

$$
\sum_{n=1}^{\infty}\left(x_{n} y_{n}^{*}+y_{n} x_{n}^{*}\right) \leq 2 \text {, }
$$

sustituindo $y_{n}^{*}=\frac{u_{n}}{y_{n}}$ e $x_{n}^{*}=\frac{u_{n}}{x_{n}}$, com $x_{n} y_{n} \neq 0$, e $u_{n} \neq 0$ na última desigualdade, obtemos que

$$
\sum_{x_{n} y_{n} \neq 0}\left(\frac{x_{n}^{2} u_{n}+y_{n}^{2} u_{n}}{x_{n} y_{n}}\right) \leq 2,
$$




\subsection{Propriedades dos indicadores}

$\log 0$

$$
\sum_{x_{n} y_{n} \neq 0}\left(\frac{x_{n}^{2} u_{n}+y_{n}^{2} u_{n}}{x_{n} y_{n}}-2 u_{n}\right) \leq 0,
$$

portanto,

$$
\sum_{x_{n} y_{n} \neq 0} u_{n}\left(\frac{x_{n}^{2}+y_{n}^{2}-2 x_{n} y_{n}}{x_{n} y_{n}}\right) \leq 0
$$

consequentemente,

$$
\sum_{x_{n} y_{n} \neq 0} u_{n} \frac{\left(x_{n}-y_{n}\right)^{2}}{x_{n} y_{n}} \leq 0 .
$$

Da última desigualdade obtemos $x_{n}=y_{n}$. Logo $x_{n}^{*}=y_{n}^{*}$.

Agora suponhamos que $u \in l_{1}$, com $\|u\|_{L_{1}}=1$ e $u_{n}=\left(u^{1}, \ldots, u^{n}, 0,0, \ldots\right) \in c_{00}$. Note que $\left|u_{n}^{k}\right| \leq u^{k}$, para todo $k \in \mathbb{N}$. Portanto $\left\|u_{n}\right\|_{l_{1}} \leq\|u\|_{l_{1}}=1$. Então existem $x_{n} \in B_{X}$ e $x_{n}^{*} \in B_{X^{*}}$, com $x_{n} \geq 0, x_{n}^{*} \geq 0$, tais que

$$
\frac{u_{n}}{\left\|u_{n}\right\|_{l_{1}}}=x_{n} \cdot x_{n}^{*}
$$

onde $u_{n}=\left\|u_{n}\right\|_{l_{1}} x_{n} \cdot x_{n}^{*}$. Se definirmos $x_{n}:=\left\|u_{n}\right\|_{l_{1}} x_{n}$ obteremos $\left\|x_{n}\right\|_{X} \leq 1$ e $u_{n}=x_{n} \cdot x_{n}^{*}$. Como $\left|x_{n}^{1}\right| \leq C_{1}\left\|x_{n}\right\|_{X} \leq C_{1}$ para todo $n \in \mathbb{N}$ (lembre-se que para todo $k \in \mathbb{N}$, o funcional $x \longmapsto x^{k}$ é contínuo), então existe uma subsequência de $\left(x_{n}^{1}\right)_{n}$ tal que $x_{n}^{1} \rightarrow y^{1}$ quando $n \rightarrow \infty$, onde $y^{1} \geq 0$. Por indução demonstramos que existe uma subsequência de $\left(x_{n}\right)_{n}$ que denotaremos por $\left(x_{n}\right)_{n}$, tal que, $x_{n}^{k} \rightarrow y^{k}$ se $n \rightarrow \infty$ para $k \in \mathbb{N}$. Assim, $x_{n}$ converge pontualmente para $y$, logo $y \in B_{X}$, pois $X$ é um E.B.S. Da mesma forma, provamos que existe uma subsequência de $\left(x_{n}^{*}\right)_{n}$, que denotaremos por $\left(x_{n}^{*}\right)_{n}$, que converge pontualmente para algum $y \in B_{X^{*}}$. Logo, $u_{n}=x_{n} \cdot x_{n}^{*}$ converge pontualmente para $y \cdot y^{*}$. Porém $u_{n}$ converge em norma de $l_{1}$ para $u$, logo $u=y \cdot y^{*}$. A unicidade pode ser provada de forma análoga ao caso $u \in c_{00}$.

Observação 6.7 Se f é uma função de valor real, então $x^{*}$ é uma função de valor real.

O seguinte resultado relaciona os indicadores de um espaço e seu dual de Köthe.

Proposição 6.8 Se X um espaço de Köthe, então

$$
\Phi_{X}(f)+\Phi_{X^{*}}(f)=\Lambda(f)
$$

para todo $f \in \mathscr{T}_{X} \cap \mathscr{T}_{X^{*}}$

Demonstração. Seja $z \in B_{L_{1}}$. Pela fatoração de Lozanovskii temos que existem $x \in B_{X}, y \in$ $B_{X^{*}}$ não negativos tais que $\frac{|z|}{\|z\|}=x y$. Logo 


$$
\begin{aligned}
\int f \log |z| d \mu & =\int f \log |x y| d \mu+\int f \log \|z\| d \mu \\
& \leq \int f \log |x| d \mu+\int f \log |y| d \mu \\
& \leq \Phi_{X}(f)+\Phi_{X^{*}}(f),
\end{aligned}
$$

portanto $\Lambda(f) \leq \Phi_{X}(f)+\Phi_{X^{*}}(f)$.

Reciprocamente, suponha $x \in B_{X}$ e $y \in B_{X^{*}}$. Pelo fato $|x y| \in L_{1}(\mu)$, temos que

$$
\begin{aligned}
\int f \log |x| d \mu+\int f \log |y| d \mu & =\int f \log |x y| d \mu \\
& \leq \Lambda(f)
\end{aligned}
$$

e então vale a desigualdade inversa.

Se $\|f\|=1$, então escrevemos $\Phi_{X} \operatorname{como} \Phi_{X}(f)=\int f \log |x| d \mu$, onde $f=x y$ é a fatoração de Lozanovskii. De fato, para $\xi \in B_{X}$

$$
\begin{aligned}
\int f \log |\xi| d \mu & =\int f \log |\xi y| d \mu-\int f \log |y| d \mu \\
& \leq \Phi_{L_{1}}(f)-\int f \log |y| d \mu \\
& =\int f \log f d \mu-\int f \log |y| d \mu \\
& =\int f \log |x| d \mu .
\end{aligned}
$$

Proposição 6.9 Sejam $X_{0}, X_{1}$ espaços de Köthe e $X_{\theta}=X_{0}^{1-\theta} X_{1}^{\theta}$ um espaço interpolado com $0<\theta<1$. Então

$$
\Phi_{X_{\theta}}(f)=(1-\theta) \Phi_{X_{0}}(f)+\theta \Phi_{X_{1}}(f)
$$

Demonstração. Seja $x \in B_{X_{\theta}}$. Existem $x_{0}$ e $x_{1}$ em $B_{X_{0}}$ e $B_{X_{1}}$ respectivamente, tais que $|x|=\left|x_{0}\right|^{1-\theta}\left|x_{1}\right|^{\theta}$. Então

$$
\begin{aligned}
\int f \log |x| d \mu & =\int f \log \left|x_{0}\right|^{\theta}\left|x_{1}\right|^{1-\theta} d \mu \\
& =(1-\theta) \int f \log \left|x_{0}\right| d \mu+\theta \int f \log \left|x_{1}\right| d \mu \\
& \leq(1-\theta) \Phi_{X_{0}}(f)+\theta \Phi_{X_{1}}(f) .
\end{aligned}
$$




\subsection{Propriedades dos indicadores}

Com isso provamos que $\Phi_{X_{\theta}}(f) \leq(1-\theta) \Phi_{X_{0}}(f)+\theta \Phi_{X_{1}}(f)$. Supondo $x_{0}$ em $B_{X_{0}}$ e $x_{1}$ em $B_{X_{1}}$, temos que

$$
\begin{aligned}
(1-\theta) \int f \log \left|x_{0}\right| d \mu+\theta \int f \log \left|x_{1}\right| d \mu & =\int f \log \left|x_{0}\right|^{\theta}\left|x_{1}\right|^{1-\theta} d \mu \\
& \leq \Phi_{X_{\theta}}(f),
\end{aligned}
$$

portanto, $\Phi_{X_{\theta}}(f) \geq(1-\theta) \Phi_{X_{0}}(f)+\theta \Phi_{X_{1}}(f)$.

Proposição 6.10 Se X um espaço de Köthe p-convexo, então $\Phi_{X^{p}}=p \Phi_{X}$.

Demonstração. Note que se $X$ é um espaço de Köthe $p$-convexo, então $X^{p}$ é um espaço de Köthe. Portanto,

$$
\begin{aligned}
\Phi_{X^{p}}(f) & =\sup _{\|x\|_{X}{ }^{p} \leq 1} \int f \log |x| d \mu \\
& =p \sup _{\|x\|_{X} \leq 1} \int f \log |x|^{1 / p} d \mu \\
& =p \sup _{\left\|\left.x\right|^{1 / p}\right\|_{X} \leq 1} \int f \log |x|^{1 / p} d \mu \\
& \leq p \sup _{\|y\|_{X} \leq 1} \int f \log |y| d \mu \\
& =p \Phi_{X}(f) .
\end{aligned}
$$

Mais ainda,

$$
\begin{aligned}
\Phi_{X}(f) & =\sup _{\|x\|_{X} \leq 1} \int f \log |x| d \mu \\
& =\frac{1}{p} \sup _{\|x\|_{X} \leq 1} \int f \log |x|^{p} d \mu \\
& =\frac{1}{p} \sup _{\left\|\left(|x|^{p}\right)^{1 / p}\right\|_{X}^{p} \leq 1} \int f \log |x|^{p} d \mu
\end{aligned}
$$




$$
\begin{aligned}
& =\frac{1}{p} \sup _{\left\||x|^{p}\right\|_{X^{p}} \leq 1} \int f \log |x|^{p} d \mu \\
& \leq \frac{1}{p} \sup _{\|y\|_{X^{p}} \leq 1} \int f \log |y| d \mu \\
& =\frac{1}{p} \Phi_{X^{p}}(f) .
\end{aligned}
$$

$\operatorname{Logo} \Phi_{X^{p}}=p \Phi_{X}$

Definição 6.11 (Funcional semilinear) Seja $\mathscr{T}$ um semi-ideal de $L_{1}^{+}$. Uma função $\Phi: \mathscr{T} \longrightarrow$ $\mathbb{C}$ é chamada de funcional semilinear, se

1. $\Phi$ é homogêneo positivo, isto é, $\Phi(\lambda f)=\lambda \Phi(f)$ para todo $\lambda \geq 0$.

2. Existe uma constante $\delta(\Phi)>0$ tal que

$$
\left|\triangle_{\Phi}(f, g)\right| \leq \delta(\Phi) \cdot\left(\|f\|_{L_{1}}+\|g\|_{L_{1}}\right),
$$

para todo $f, g \in \mathscr{T}$, onde $\Delta_{\Phi}(f, g)=\Phi(f)+\Phi(g)-\Phi(f+g)$.

3. Se $f \in \mathscr{T}$ e $\left(f_{n}\right)_{n}$ é uma sequência de $\mathscr{T}$ tal que $0 \leq f_{n} \leq f$ para todo $n \in \mathbb{N}$, com $\lim _{n \rightarrow \infty}\left\|f_{n}\right\|_{L_{1}}=0$, então $\lim _{n \rightarrow \infty} \Phi\left(f_{n}\right)=0$.

Vamos denotar como $\delta(\Phi)$ a menor constante que verifica essa condição .

Segue de forma imediata da definição que se $\Phi, \Psi$ são funcionais lineares e $\lambda$ é um número real, então $\lambda \Phi+\Psi$ é um funcional semilinear.

Observação 6.12 Vamos denotar por

$$
\Delta_{\Phi}\left(f_{1}, \ldots, f_{n}\right)=\sum_{k=1}^{n} \Phi\left(f_{k}\right)-\Phi\left(\sum_{k=1}^{n} f_{k}\right) .
$$

É imediato provar que se $\Phi, \Psi: \mathscr{T} \longrightarrow \mathbb{R}$ são funcionais homogêneos e $\lambda \in \mathbb{R}$, então

$$
\Delta_{\Psi+\lambda \Phi}\left(f_{1}, \ldots, f_{n}\right)=\Delta_{\Psi}\left(f_{1}, \ldots, f_{n}\right)+\lambda \Delta_{\Phi}\left(f_{1}, \ldots, f_{n}\right) .
$$

Por indução, prova-se que

$$
\left|\Delta_{\Psi}\left(f_{1}, \ldots, f_{n}\right)\right| \leq(n-1) \delta(\Phi) \sum_{k=1}^{n}\left\|f_{k}\right\|_{L_{1}} .
$$


Definição 6.13 (Funcional convexo e côncavo ) Um funcional $\Phi: \mathscr{T} \longrightarrow \mathbb{R}$ homogêneo positivo será chamado de convexo se, e somente se, $\Delta_{\Phi}(f, g) \geqslant 0$. O funcional $\Phi$ será chamado de côncavo se, e somente se, $\Delta_{\Phi}(f, g) \leqslant 0$.

Note que a soma de funcionais convexos (respectivamente côncavo) é convexa respectivamente côncavo).

Definição 6.14 (Funcional $K$-contínuo) Um funcional semilinear $\Phi: \mathscr{T} \longrightarrow \mathbb{C}$ é dito $K$ contínuo, se

$$
\lim _{\varepsilon \rightarrow 0} \sup _{\|f\|_{L_{1}} \leq 1} \sup _{\|g\|_{L_{1}} \leq \varepsilon}\left|\Delta_{\Phi}(f, g)\right|=0
$$

Teorema 6.15 Seja $X$ um espaço de funções g-convexo. Então $\Phi_{X}$ é um funcional semilinear, $K$-contínuo e convexo. Se X é um espaço de Köthe, então $\delta\left(\Phi_{X}\right) \leq \log 2$.

Demonstração. Demonstraremos o teorema por partes.

Passo 1. Afirmamos que $\Phi_{X}$ é um funcional semilinear.

Passo 1.A. $\Phi_{X}$ é homogêneo positivo. Imediato.

Passo 1.B. Provaremos que $\Phi_{X}$ verifica 2 . da definição de funcional semilinear.

Sejam $f, g \in \mathscr{T}_{X}$ e $\varepsilon>0$. Então existem $x$ e $y \in B_{X}$ tais que

$$
\int f \log |x| d \mu \geq \Phi_{X}(f)-\frac{\varepsilon}{2}
$$

$\mathrm{e}$

$$
\int g \log |y| d \mu \geq \Phi_{X}(g)-\frac{\varepsilon}{2}
$$

Para $0<\tau<\infty$, definamos

$$
v_{\tau}=(1+\eta(\tau))^{-1} \max (|x|, \tau|y|)
$$

onde $\eta(\tau)$ é o número introduzido em Definição 5.4. Note que $v_{\tau} \in B_{X}$ pois se $A=\{s:|x(s)| \geq$ $\tau|y(s)|\}$ e $B=\{s:|x(s)|<\tau|y(s)|\}$. Então pelo fato $\operatorname{supp}\left(|x| 1_{A}\right) \cap \operatorname{supp}\left(\tau|y| 1_{B}\right)=\emptyset$, temos que

$$
\begin{aligned}
\|\max (|x|, \tau|y|)\|_{X} & =\left\||x| 1_{A}+\tau|y| 1_{B}\right\|_{X} \\
& \leq 1+\eta(\tau) .
\end{aligned}
$$

Mas ainda, 


$$
\begin{aligned}
\Phi_{X}(f+g) & \geq \int(f+g) \log \left|v_{\tau}\right| d \mu \\
& =\int f \log \left|v_{\tau}\right| d \mu+\int g \log \left|v_{\tau}\right| d \mu \\
& \geq \int f \log (1+\eta(\tau))^{-1}|x| d \mu+\int g \log \tau(1+\eta(\tau))^{-1}|y| d \mu \\
& \geq \Phi_{X}(f)+\Phi_{X}(g)-\varepsilon-\|f\|_{L_{1}} \log (\eta(\tau)+1)-\|g\|_{L_{1}} \log (\eta(\tau)+1)+\|g\|_{L_{1}} \log \tau
\end{aligned}
$$

para todo $\varepsilon>0$, isto é,

$$
\Delta_{\Phi_{X}}(f, g) \leq\left(\|f\|_{L_{1}}+\|g\|_{L_{1}}\right) \log (\eta(\tau)+1)-\|g\|_{L_{1}} \log \tau,
$$

para todo $\tau>0$ e todo $f, g \in \mathscr{T}_{X}$. Em particular, se $\tau=1$, temos que $\Phi_{X}$ verifica 2 . da definição de funcional semilinear. No Passo 2. demostramos que $0 \leq \Delta_{\Phi_{X}}(f, g)$ para todo $f, g \in \mathscr{T}_{X}$.

Passo 1.C. Agora demonstremos que dados $f \in \mathscr{T}_{X}$ e $\left(f_{n}\right)_{n}$ uma sequência de $\mathscr{T}_{X}$ tais que $0 \leq f_{n} \leq f$ para todo $n \in \mathbb{N}$, com $\lim _{n \rightarrow \infty}\left\|f_{n}\right\|_{L_{1}}=0$, temos que $\lim _{n \rightarrow \infty} \Phi_{X}\left(f_{n}\right)=0$ (parte 3 . da definição de funcional semilinear). De fato, definamos o espaço

$$
F=\left\{g \in L_{1}:\|g\|_{F}=\sup _{x \in B_{X}} \int|g| \log _{+}|x| d \mu+\|g\|_{L_{1}}<\infty\right\} .
$$

$\operatorname{Logo}\left(F,\|\cdot\|_{F}\right)$ é um espaço normado e verifica:

$$
|h| \leq|g| \Rightarrow\|h\|_{F} \leq\|g\|_{F} .
$$

Mostraremos que $F$ é um espaço com norma de ordem contínua (Ver Subseção 2.3.2). Pela Proposição 2.45 basta verificar que dadas $g \geq 0$ e uma sequência de conjuntos mensuráveis disjuntos $\left(S_{n}\right)_{n}$, temos que $\left\|g \cdot 1_{S_{n}}\right\|_{F} \rightarrow 0$, se $n \rightarrow \infty$. Como $L_{1}$ é um espaço com norma de ordem contínua, segue-se que $\left\|g \cdot 1_{S_{n}}\right\|_{L_{1}} \rightarrow 0$. Portanto é suficiente verificar que

$$
\sup _{x \in B_{X}} \int\left|g \cdot 1_{S_{n}}\right| \log _{+}|x| d \mu \rightarrow 0 \text {, se } n \rightarrow \infty .
$$

Suponha por contradição que

$$
\sup _{x \in B_{X}} \int\left|g \cdot 1_{S_{n}}\right| \log _{+}|x| d \mu \rightarrow 0
$$




\subsection{Propriedades dos indicadores}

isto é, existe uma sequência de conjuntos mensuráveis disjuntos $\left(S_{n}\right)_{n}$ e $\varepsilon>0$ tais que

$$
\sup _{x \in B_{X}} \int_{S_{n}} g \log _{+}|x| d \mu>\varepsilon .
$$

Portanto para cada $n \in \mathbb{N}$, existe $x_{n} \in B_{X}$ tal que

$$
\varepsilon<\int_{S_{n}} g \log _{+}\left|x_{n}\right| d \mu .
$$

Vamos definir a sequência $y_{n}=\max \left(\left|x_{1}\right|,\left|x_{2}\right|, \ldots,\left|x_{n}\right|\right)$. Agora, escolhendo $\rho>0$ e $\beta>1$ oportunos obtemos que $\left\|y_{n}\right\|_{X} \leq \beta n^{1 / \rho}$. Isto é, $\beta^{-1} n^{-1 / \rho} y_{n} \in B_{X}$. Portanto,

$$
\begin{aligned}
\Phi_{X}(g) & \geq \int g \log _{+}\left|\beta^{-1} n^{-1 / \rho} y_{n}\right| d \mu \\
& =\int g \log _{+}\left|y_{n}\right| d \mu-\|g\|_{L_{1}}\left(\log \beta+\frac{1}{\rho} \log n\right) \\
& \geq \sum_{k=1}^{n} \int_{S_{k}} g \log _{+}\left|y_{n}\right| d \mu-\|g\|_{L_{1}}\left(\log \beta+\frac{1}{\rho} \log n\right) \\
& \geq \sum_{k=1}^{n} \int_{S_{k}} g \log _{+}\left|x_{k}\right| d \mu-\|g\|_{L_{1}}\left(\log \beta+\frac{1}{\rho} \log n\right) \\
& \geq n \varepsilon-\|g\|_{L_{1}}\left(\log \beta+\frac{1}{\rho} \log n\right),
\end{aligned}
$$

para todo $n \in \mathbb{N}$, o que contraria o fato de $\operatorname{ser} n \varepsilon-\|g\|_{L_{1}}\left(\log \beta+\frac{1}{\rho} \log n\right)$ uma sequência não limitada. Daqui concluimos que $F$ é um espaço com norma de ordem contínua.

Afirmamos que se $\|\cdot\|_{F}$ é uma norma de ordem contínua, então $\left\|f_{n}\right\|_{F} \rightarrow 0$. De fato, suponha por contradição que $\left\|f_{n}\right\|_{F} \nrightarrow 0$, isto é, existe uma subsequência de $\left(f_{n}\right)_{n}$, que denotaremos por $\left(f_{n}^{\prime}\right)_{n}$, e $\varepsilon>0$, tal que $\left\|f_{n}^{\prime}\right\|_{F}>\varepsilon$ para todo $n \in \mathbb{N}$. Agora, por hipótese $\left\|f_{n}^{\prime}\right\|_{L_{1}} \rightarrow 0$. Portanto existe uma subsequência de $\left(f_{n}^{\prime}\right)_{n}$, que denotamos por $\left(f_{n}^{\prime \prime}\right)_{n}$, tal que $f_{n}^{\prime \prime} \rightarrow 0$ q.s. Se definirmos $g_{n}(s)=\sup _{k \geq n} f_{k}^{\prime \prime}(s)$, temos que $\left(g_{n}\right)_{n}$ é uma sequência decrescente de $F$, com $g_{n} \rightarrow 0$ q.s. e $f_{n}^{\prime \prime} \leq g_{n}$. Como $\|\cdot\|_{F}$ é uma norma de ordem contínua, obtemos que $\left\|g_{n}\right\|_{F} \rightarrow 0$. Usando a afirmação (6.10), deduzimos que $\left\|f_{n}^{\prime \prime}\right\|_{F} \rightarrow 0$, o qual é absurdo. Portanto, $\left\|f_{n}\right\|_{F} \rightarrow 0$, isto é,

$$
\sup _{x \in B_{X}} \int f_{n} \log _{+}|x| d \mu \rightarrow 0,
$$

e como

$$
\Phi_{X}\left(f_{n}\right) \leq \sup _{x \in B_{X}} \int f_{n} \log _{+}|x| d \mu,
$$




\subsection{Propriedades dos indicadores}

temos que

$$
\limsup _{n \rightarrow \infty} \Phi_{X}\left(f_{n}\right) \leq 0 .
$$

Por outro lado, como $f \in \mathscr{T}_{X}$, então existe $x \in B_{X}$ tal que $f|\log | x||$ é integrável. Logo $f_{n}|\log | x|| \leq f|\log | x||$. O Teorema da Convergência Dominada implica que $\lim _{n \rightarrow \infty} \int f_{n}|\log | x|| d \mu=$ 0 , isto é, $\lim _{n \rightarrow \infty}\left|\int f_{n} \log \right| x|d \mu|=0$. Como

$$
\int f_{n} \log |x| d \mu \leq \Phi_{X}\left(f_{n}\right)
$$

temos que

$$
\liminf _{n \rightarrow \infty} \Phi_{X}\left(f_{n}\right) \geq 0 .
$$

Com isso, nós provamos que $\lim _{n \rightarrow \infty} \Phi_{X}\left(f_{n}\right)=0$. Note que até o momento nós demonstramos que $\Phi_{X}$ é semilinear.

Passo 2. Provaremos que $\Phi_{X}$ é convexo. De fato, seja $x$ em $B_{X}$. Logo

$$
\begin{aligned}
\int(f+g) \log |x| d \mu & =\int f \log |x| d \mu+\int g \log |x| d \mu \\
& \leq \Phi_{X}(f)+\Phi_{X}(g)
\end{aligned}
$$

portanto

$$
\int(f+g) \log |x| d \mu \leq \Phi_{X}(f)+\Phi_{X}(g),
$$

para todo $x \in B_{X}$. Logo $\Delta_{\Phi_{X}}(f, g) \geq 0$ para todo $f, g \in \mathscr{T}_{X}$.

Passo 3. Provaremos que $\Phi_{X}$ é $K$-contínuo. De fato, no Passo 1., nós provamos que $\Phi_{X}$ verifica

$$
\Delta_{\Phi_{X}}(f, g) \leq\left(\|f\|_{L_{1}}+\|g\|_{L_{1}}\right) \log (\eta(\tau)+1)-\|g\|_{L_{1}} \log \tau .
$$

(ver desigualdade (6.9)).

Supondo $\|f\|_{L_{1}} \leq 1$ e $\|g\|_{L_{1}} \leq \varepsilon$, obtemos que

$$
0 \leq \sup _{\|f\|_{L_{1}} \leq 1} \sup _{\|g\|_{L_{1}} \leq \varepsilon} \Delta_{\Phi_{X}}(f, g) \leq(1+\varepsilon) \log (\eta(\tau)+1)-\varepsilon \log \tau,
$$

portanto

$$
0 \leq \lim _{\varepsilon \rightarrow 0} \sup _{\|f\|_{L_{1}} \leq 1} \sup _{\|g\|_{L_{1}} \leq \varepsilon} \Delta_{\Phi_{X}}(f, g) \leq \log (\eta(\tau)+1)
$$




\subsection{Propriedades dos indicadores}

se $\tau \rightarrow 0$, obtemos que

$$
\lim _{\varepsilon \rightarrow 0} \sup _{\|f\|_{L_{1}} \leq 1} \sup _{\|g\|_{L_{1}} \leq \varepsilon} \Delta_{\Phi_{X}}(f, g)=0
$$

Passo 4. No caso em que $X$ seja um espaço de Köthe, obtemos que $\eta(\tau) \leq \tau$ (ver Observação 5.5, parte 3.), logo da desigualdade

$$
\Delta_{\Phi_{X}}(f, g) \leq\left(\|f\|_{L_{1}}+\|g\|_{L_{1}}\right) \log (\eta(\tau)+1)-\|g\|_{L_{1}} \log \tau,
$$

para todo $\tau>0$ e todo $f, g \in \mathscr{T}_{X}$, demonstrada no Passo 1. (ver desigualdade (6.9) ) temos que

$$
\Delta_{\Phi_{X}}(f, g) \leq\left(\|f\|_{L_{1}}+\|g\|_{L_{1}}\right) \log (\tau+1)-\|g\|_{L_{1}} \log \tau,
$$

para todo $f, g \in \mathscr{T}_{X}$. Se $\tau=1$, então

$$
\Delta_{\Phi_{X}}(f, g) \leq \log 2 \cdot\left(\|f\|_{L_{1}}+\|g\|_{L_{1}}\right)
$$

para todo $f, g \in \mathscr{T}_{X}$, isto é, $\delta\left(\Phi_{X}\right) \leq \log 2$.

Teorema 6.16 Seja $\mathscr{T}$ um semideal e $\Phi$ um funcional semilinear em $\mathscr{T}$. Então

- Para todo $f \in \mathscr{T}, \Phi$ é limitado em $[0, f]$.

- Se $f_{n} \in[0, f] e \lim _{n \rightarrow \infty}\left\|f_{n}-g\right\|_{L_{1}}=0$, então

$$
\limsup _{n \rightarrow \infty}\left|\Phi\left(f_{n}\right)-\Phi(g)\right| \leq 2 \delta(\Phi) \cdot\|f\|_{L_{1}}
$$

- Se $\Phi$ é K-contínuo, então $\Phi$ é contínuo em $[0, f]$ com a norma $\|\cdot\|_{L_{1}}$.

\section{Demonstração.}

- Sejam $f \in \mathscr{T}, \Gamma(f)=\sup \{|\Phi(g)|: 0 \leq g \leq f\}$. Vejamos que $\Gamma(f)<\infty$. Suponhamos que $\Gamma(f)=\infty$. Então existe $g \in[0, f]$ tal que

$$
|\Phi(g)|>3|\Phi(f)|+\delta(\Phi) \cdot\|f\|_{L_{1}}+1 .
$$

Agora, pelo fato de $\Phi$ ser semilinear e denotando $\delta:=\delta(\Phi)$, temos que

$$
|\Phi(f-g)+\Phi(g)-\Phi(f)| \leq \delta\left(\|f-g\|_{L_{1}}+\|g\|_{L_{1}}\right)=\delta\|f\|_{L_{1}},
$$

o que implica

$$
|\Phi(g)|-|\Phi(f)|-\delta\|f\|_{L_{1}} \leq|\Phi(f-g)| .
$$

Note que 


\subsection{Propriedades dos indicadores}

$$
\begin{aligned}
2|\Phi(f)|+1 & =\left(3|\Phi(f)|+\delta\|f\|_{L_{1}}+1\right)-|\Phi(f)|-\delta\|f\|_{L_{1}} \\
& <|\Phi(g)|-|\Phi(f)|-\delta\|f\|_{L_{1}} \\
& \leq|\Phi(f-g)| .
\end{aligned}
$$

Resumindo, existe $g \in[0, f]$ tal que $2|\Phi(f)|+1<|\Phi(f-g)|$.

Seja $f_{0}=f$. Então, pelo fato anterior, existe $g_{0} \geq 0$, com $g_{0} \leq f_{0}$, tal que $2\left|\Phi\left(f_{0}\right)\right|+1<$ $\left|\Phi\left(f_{0}-g_{0}\right)\right|$. Se $f_{1}=f_{0}-g_{0}$, obtemos $f_{1} \leq f_{0}$ e $2\left|\Phi\left(f_{0}\right)\right|+1<\left|\Phi\left(f_{1}\right)\right|$. Por indução, encontramos uma sequência decrescente de funções não negativas em $[0, f]$, que denotaremos por $\left(f_{n}\right)_{n}$, tal que $2\left|\Phi\left(f_{n}\right)\right|+1<\left|\Phi\left(f_{n-1}\right)\right|$. Portanto verifica-se $\left|\Phi\left(f_{n}\right)\right|>2^{n}|\Phi(f)|+2^{n}+1$ para todo $n \in \mathbb{N}$ e assim $\lim _{n \rightarrow \infty}\left|\Phi\left(f_{n}\right)\right|=\infty$. Pelo fato de que $\left(f_{n}\right)_{n}$ é uma sequência de funções decrescente, temos que que existe uma função $g \geq 0$ em $[0, f]$ tal que $f_{n} \rightarrow g$ q.s., se $n \rightarrow \infty$. Pelo Teorema da Convergência Monótona, obtemos que $\left\|f_{n}-g\right\|_{L_{1}} \longrightarrow 0$, se $n \rightarrow \infty$. Mas ainda, pelas desigualdades $0 \leq f_{n}-g \leq f-g$ e por $\Phi$ ser semilinear, temos que $\lim _{n \rightarrow \infty} \Phi\left(f_{n}-g\right)=0$. Agora,

$$
\begin{aligned}
\left|\Phi\left(f_{n}\right)\right| & \leq\left|\Phi(g)+\Phi\left(f_{n}-g\right)-\Phi\left(f_{n}\right)\right|+\left|\Phi\left(f_{n}-g\right)\right|+|\Phi(g)| \\
& \leq \delta\left\|f_{n}\right\|_{L_{1}}+\left|\Phi\left(f_{n}-g\right)\right|+|\Phi(g)| \\
& \leq \delta\|f\|_{L_{1}}+\left|\Phi\left(f_{n}-g\right)\right|+|\Phi(g)| .
\end{aligned}
$$

Isto prova que $\left(\left|\Phi\left(f_{n}\right)\right|\right)_{n}$ é limitada, o qual é contraditório.

- Seja $h_{n}=\max \left(f_{n}, g\right)$. Então,

$$
\left|\Phi\left(h_{n}\right)-\Phi\left(f_{n}\right)-\Phi\left(h_{n}-f_{n}\right)\right| \leq \delta\left\|h_{n}\right\|_{L_{1}} \leq \delta\|f\|_{L_{1}} .
$$

Similarmente

$$
\left|\Phi\left(h_{n}\right)-\Phi(g)-\Phi\left(h_{n}-g\right)\right| \leq \delta\|f\|_{L_{1}} .
$$

Agora pela desigualdade triangular e as desigualdade anteriores, temos que

$$
\left|\Phi\left(f_{n}\right)-\Phi(g)\right| \leq\left|\Phi\left(h_{n}-f_{n}\right)\right|+\left|\Phi\left(h_{n}-g\right)\right|+2 \delta\|f\|_{L_{1}} .
$$

Observe que $\left|h_{n}(s)-f_{n}(s)\right| \leq\left|g_{n}(s)-f_{n}(s)\right|$ para todo $s \in S$, logo $\left\|h_{n}-f_{n}\right\|_{L_{1}} \leq\left\|g_{n}-f_{n}\right\|_{L_{1}} \mathrm{e}$ então $\left\|h_{n}-f_{n}\right\|_{L_{1}} \rightarrow 0$. Similarmente provamos $\left\|h_{n}-g\right\|_{L_{1}} \rightarrow 0$. Agora como $h_{n}-g \leq f-g$, então pela hipótese de $\Phi$ ser semilinear, obtemos que

$$
\lim _{n \rightarrow \infty} \Phi\left(h_{n}-f_{n}\right)=\lim _{n \rightarrow \infty} \Phi\left(h_{n}-g\right)=0
$$




\subsection{Propriedades dos indicadores}

concluindo esta parte do teorema.

- Suponha que $\Phi$ seja $K$-contínuo, isto é,

$$
\lim _{\varepsilon \rightarrow 0} \sup _{\|f\|_{L_{1}} \leq 1} \sup _{\|g\|_{L_{1}} \leq \varepsilon}\left|\Delta_{\Phi}(f, g)\right|=0
$$

Seja $g \in[0, f]$ fixo e $\left(f_{n}\right)_{n}$ uma sequência em $[0, f]$ tal que $\left\|f_{n}-g\right\|_{L_{1}} \rightarrow 0$. Mostremos que $\lim _{n \rightarrow \infty}\left(\Phi\left(f_{n}\right)-\Phi(g)\right)=0$. Assumamos primeiro que $\|f\|_{L_{1}} \leq 1, \log$ o $\left\|f_{n}\right\|_{L_{1}} \leq 1$. Seja $h_{n}=\max \left(f_{n}, g\right)$ como na parte anterior. Analogamente, como no item 2 do teorema, obtemos que $\left\|h_{n}-f_{n}\right\|_{L_{1}} \rightarrow 0$. Para $n \in \mathbb{N}$ e $\varepsilon_{n}=\left\|h_{n}-f_{n}\right\|_{L_{1}}$, temos que

$$
\left|\Delta_{\Phi}\left(f_{n}, h_{n}-f_{n}\right)\right| \leq \sup _{\|F\|_{L_{1}} \leq 1} \sup _{\|G\|_{L_{1}} \leq \varepsilon_{n}}\left|\Delta_{\Phi}(F, G)\right|,
$$

usando a hipóteses concluimos que

$$
\lim _{n \rightarrow \infty}\left|\Delta_{\Phi}\left(f_{n}, h_{n}-f_{n}\right)\right|=0 .
$$

Cálculos similares demonstram que $\lim _{n \rightarrow \infty}\left|\Delta_{\Phi}\left(g, h_{n}-g\right)\right|=0$. Usando a desigualdade triangular, deduzimos que

$$
\left|\Phi\left(f_{n}\right)-\Phi(g)\right| \leq\left|\Delta_{\Phi}\left(f_{n}, h_{n}-f_{n}\right)\right|+\left|\Delta_{\Phi}\left(g, h_{n}-g\right)\right|,
$$

obtendo que $\lim _{n \rightarrow \infty}\left|\Phi\left(f_{n}\right)-\Phi(g)\right|=0$.

Agora assumamos $\|f\|_{L_{1}}>1$. Considerando $\frac{f}{\|f\|_{L_{1}}}$ e aplicando o caso anterior, temos que

$$
\lim _{n \rightarrow \infty}\left|\Phi\left(\frac{f_{n}}{\|f\|_{L_{1}}}\right)-\Phi\left(\frac{g}{\|f\|_{L_{1}}}\right)\right|=0
$$

E como $\Phi$ é homogêneo positivo, obtemos que $\lim _{n \rightarrow \infty}\left|\Phi\left(f_{n}\right)-\Phi(g)\right|=0$.

Teorema 6.17 Seja X um espaço de funções g-convexo e $\mathscr{T}$ um semi-ideal estrito, com $\mathscr{T} \subseteq$ $\mathscr{T}_{X}$. Então $x \in B_{X}$ se, e somente se, $f \log _{+}|x|$ é integrável $e$

$$
\int f \log |x| d \mu \leq \Phi_{X}(f) \text { para todo } f \in \mathscr{T} \text {. }
$$

Demonstração. A prova da ida é imediata pela definição de $\mathscr{T}_{X}$. 


\subsection{Propriedades dos indicadores}

Passo 1. Vamos provar a recíproca do teorema.

Passo 1.A. Vamos mostrar que é possível assumir, sem perda de generalidade, que $\mathscr{T}=\mathscr{T}_{X}$. A ideia é provar que se o teorema é válido para $\mathscr{T}_{X}$, então o teorema também é válido para todo semi-ideal estrito $\mathscr{T}$. De fato, suponha que a recíproca do teorema é válido para $\mathscr{T}_{X}$ e que $f \log _{+}|x|$ é integrável para todo $f \in \mathscr{T}$ e

$$
\int f \log |x| d \mu \leq \Phi_{X}(f) \text { para todo } f \in \mathscr{T} \text {. }
$$

Vamos demonstrar que $x \in B_{X}$. Seja $f \in \mathscr{T}_{X}$. Como $\mathscr{T}$ é um semi-ideal estrito, então existe um $g$ positivo em $\mathscr{T}$. Definamos uma sequência de funções como $f_{n}=\min (n g, f)$. Observe que $0 \leq f_{n} \leq n g$, onde $n g \in \mathscr{T}$. Portanto $\left(f_{n}\right)_{n}$ é uma sequência crescente de $\mathscr{T}$, com $f_{n} \rightarrow f$ q.s. Agora, seja $A=\{s:|x(s)|>1\}$. Pelo fato que $f_{n} \in \mathscr{T}$, temos que $1_{A} f_{n} \in \mathscr{T}$. Logo

$$
\int f_{n} 1_{A} \log |x| d \mu \leq \Phi_{X}\left(f_{n} 1_{A}\right)
$$

O Teorema da Convergência Monótona implica que $\left\|f_{n} 1_{A}-f 1_{A}\right\|_{L_{1}} \rightarrow 0$, logo pelos Teoremas 6.15 e 6.16 , temos que $\Phi_{X}\left(f_{n} 1_{A}\right) \rightarrow \Phi_{X}\left(f 1_{A}\right)$, se $n \rightarrow \infty$. Novamente, o Teorema da Convergência Monótona implica

$$
\int f \log _{+}|x| d \mu=\lim _{n \rightarrow \infty} \int f_{n} 1_{A} \log |x| d \mu \leq \Phi_{X}\left(f 1_{A}\right),
$$

e portanto $\int f \log _{+}|x|$ é integrável para todo $f \in \mathscr{T}_{X}$. Agora, se $\int f \log |x| d \mu=-\infty$, então imediatamente $\int f \log |x| d \mu \leq \Phi_{X}(f)$. Assuma agora que $f \log |x| \in L_{1}$. Então pelo teorema Convergência Dominada e os Teoremas 6.15,6.16, temos que

$$
\begin{aligned}
\int f \log |x| d \mu & =\lim _{n \rightarrow \infty} \int f_{n} \log |x| d \mu \\
& \leq \lim _{n \rightarrow \infty} \Phi_{X}\left(f_{n}\right) \\
& =\Phi(f)
\end{aligned}
$$

para todo $f \in \mathscr{T}_{X}$. Como a recíproca do teorema vale para $\mathscr{T}_{X}$, temos que $x \in B_{X}$. Isto mostra que podemos assumir que $\mathscr{T}=\mathscr{T}_{X}$.

Passo 2. Demonstraremos a recíproca do teorema para $\mathscr{T}_{X}$. Suponha que $f \log _{+}|x|$ é integrável para todo $f \in \mathscr{T}_{X} \mathrm{e}$

$$
\int f \log |x| d \mu \leq \Phi_{X}(f) \text { para todo } f \in \mathscr{T}_{X}
$$




\subsection{Propriedades dos indicadores}

Provemos que $x \in B_{X}$.

Assuma por contradição que $x \notin B_{X}$. Como $X$ é um espaço de funções g-convexo, então existe $u$ estritamente positiva em $B_{X}$. Agora, existe $w$ estritamente positiva em $L_{0}$ tal que

$$
\int w(1+|\log | x||+|\log u|)^{2} d \mu<\infty .
$$

Seja $L_{2}(w d \mu)$ o espaço de Hilbert de todos os $\phi$ tais que

$$
\|\phi\|_{w, 2}^{2}=\int|\phi|^{2} w d \mu<\infty .
$$

Seja $M=\left\{\log |y|: y \in B_{X}\right\}$ e $V=L_{2}(w d \mu) \cap M$. De (6.11), obtemos que $\int w|\log u|^{2} d \mu<\infty$. Logo $V \neq \emptyset$, pois $\log u \in V$.

Demonstraremos agora que $V$ é convexo. Sejam $\log |y|, \log |z| \in V$ e $0 \leq t \leq 1$. Então $(1-t) \log |y|+t \log |z|=\log |y|^{1-t}|z|^{t}$, como $X$ é um espaço de funções g-convexo, temos que $\left|\left\|\left.y\right|^{1-t}|z|^{t}\right\|_{X} \leq\|y\|_{X}^{1-t}\|z\|_{X}^{t} \leq 1\right.$. Portanto $\left.(1-t) \log \right| y|+t \log | z \mid \in V$.

Agora Provemos que $V$ é fechado em $L_{2}(w d \mu)$. De fato, seja $\left(f_{n}\right)_{n}$ uma sequência em $V$, onde $f_{n}=\log \left|y_{n}\right|$, com $y_{n} \in B_{X}$ e $f \in L_{2}(w d \mu)$, tal que $\left\|f_{n}-f\right\|_{w, 2} \rightarrow 0$, se $n \rightarrow \infty$. Portanto, existe uma subsequência de $\left(f_{n}\right)_{n}$, que denotamos por $\left(f_{n}\right)_{n}$, tal que $\log \left|y_{n}\right|=f_{n} \rightarrow f$ q.s., isto é, $\left|y_{n}\right| \rightarrow e^{f}$ q.s., portanto, $\left|y_{n}\right| \rightarrow e^{f}$ em medida, como $B_{X}$ é fechada nesta convergência, temos que $e^{f} \in B_{X}$, logo, $f \in V$. Observe que $V$ verifica:

$$
\phi_{1} \in V, \phi_{2} \in L_{2}(w d \mu) \text { e } \phi_{2} \leq \phi_{1} \Rightarrow \phi_{2} \in V .
$$

Como $\log |x| \notin V$, o teorema de separação de Hahn-Banach (ver Teorema 2.66) garante a existência de um número real $\alpha$ e um funcional linear $\varphi: L_{2}(w d \mu) \longrightarrow \mathbb{C}$ tais que

$$
\operatorname{Re}(\varphi(\log |x|))>\alpha \geq \operatorname{Re}(\varphi(\phi))
$$

para todo $\phi \in V$. Agora existe $G \in L_{2}\left(w^{-1} d \mu\right)$ tal que $\varphi(\phi)=\int G \phi d \mu$ para todo $\phi \in L_{2}(w d \mu)$. $\operatorname{Se} \operatorname{Re}(G)=g$, então $\operatorname{Re}(\varphi(\phi))=\int g \phi d \mu$ para todo $\phi \in V$ e $\operatorname{Re}(\varphi(\log |x|))=\int g \log |x| d \mu$, assim

$$
\int g \log |x| d \mu>\alpha \geq \int g \phi d \mu
$$

para todo $\phi \in V$.

Passo 2.A. Demonstraremos que $g \in \mathscr{T}_{X}$. De fato,

Primeiro demonstraremos que $g \geq 0$ q.s. Suponha por contradição que o conjunto $I=\{s$ : $g(s)<0\}$ não tem medida zero. Definamos para cada $n \in \mathbb{N}$ uma função em $S$ por

$$
\lambda_{n}(s)= \begin{cases}1 / n, & \text { se } s \in I \\ 1 / 2, & \text { se } s \in S \backslash I .\end{cases}
$$




\subsection{Propriedades dos indicadores}

Observe que $\log \lambda_{n} u \leq \log u$, portanto $\log \lambda_{n} u \in L_{2}(w d \mu)$ (lembre-se que $\log u \in V$ ), assim pela propriedade 6.12 que $V$ verifica, obtemos que $\log \lambda_{n} u \in V$, portanto $\int g \log \lambda_{n} u d \mu \leq \alpha$ para todo $n \in \mathbb{N}$. Agora

$$
\begin{aligned}
\int g \log \lambda_{n} u d \mu \leq \alpha & \Leftrightarrow \int_{I} g \log \lambda_{n} u d \mu+\int_{S \backslash I} g \log \lambda_{n} u d \mu \leq \alpha \\
& \Leftrightarrow \log \frac{1}{n} \int_{I} g d \mu+\int_{I} g \log u d \mu+\int_{S \backslash I} g \log \frac{u}{2} d \mu \leq \alpha,
\end{aligned}
$$

para todo $n \in \mathbb{N}$.

Os cálculos acimas mostram que a sequência não negativa $\left(\log \frac{1}{n} \int_{I} g d \mu\right)$ é limitada, o qual é absurdo.

Afirmamos que $g \in L_{1}(\mu)$. Observe que $g=g w^{-1 / 2} \cdot w^{1 / 2}$, onde $g w^{-1 / 2} \in L_{2}(\mu)$ e $w^{1 / 2} \in L_{2}(\mu)$ (isto é consequência de (6.11)). A desigualdade de Hölder prova que $g \in L_{1}(\mu)$.

Agora verifiquemos que

$$
\int g|\log | u|| d \mu<\infty
$$

$\mathrm{e}$

$$
\sup _{y \in B_{X}} \int g \log _{+}|y|<\infty
$$

para qualquer $y \in B_{X}$. De fato:

Para demonstrar a primeira parte, lembre-se que $g|\log u|=g w^{-1 / 2} \cdot\left(w^{1 / 2}|\log u|\right)$, onde $g w^{-1 / 2} \mathrm{e}$ $w^{1 / 2}|\log u|$ pertencem a $L_{2}(\mu)$. Logo $g|\log u| \in L_{1}(\mu) \mathrm{e}$

$$
\int g|\log u| d \mu \leq\|g\|_{w^{-1}, 2} \cdot\|\log u\|_{w, 2}
$$

Para demonstrar a segunda parte, seja $y \in B_{X}$ e definamos

$$
y_{m, \tau}=\max (\min (|y|, m|x|), \tau|u|),
$$

para $m \in \mathbb{N}$ e $\tau>0$. Note que $(\eta(\tau)+1)^{-1} y_{m, \tau} \in B_{X}$, onde $\eta(\tau)$ é o número definido na Definição $5.4 \mathrm{e}$

$$
\left.\log (\eta(\tau)+1)^{-1} y_{m, \tau}=\log y_{m, \tau}-\log (\eta(\tau)+1)\right) \in L_{2}(w d \mu)
$$

pois

$$
\log \tau u \leq \log y_{m, \tau} \leq \log \max (m|x|, \tau u)=1_{A} \cdot \log m|x|+1_{B} \cdot \log \tau u,
$$




\subsection{Propriedades dos indicadores}

onde

$$
A=\{s: m|x(s)| \geq \tau|u(s)|\} \text { e } B=\{s: m|x(s)|<\tau u(s)\} .
$$

Resumindo temos que

$$
\log \tau u \leq \log y_{m, \tau} \leq 1_{A} \cdot \log m|x|+1_{B} \cdot \log \tau u,
$$

onde $\log \tau u$ e $1_{A} \cdot \log m|x|+1_{B} \cdot \log \tau u$ são funções de $L_{2}(w d \mu)$, (isto é deduzido de (6.11)). Portanto $\log y_{m, \tau} \in L_{2}(w d \mu)$. Logo, concluimos que $\left.\log y_{m, \tau}-\log (\eta(\tau)+1)\right) \in L_{2}(w d \mu)$. Isto mostra que

$$
\log y_{m, \tau}(\eta(\tau)+1)^{-1} \in V
$$

Logo

$$
\int g \log y_{m, \tau}(\eta(\tau)+1)^{-1} d \mu \leq \alpha
$$

que equivale a

$$
\int g \log y_{m, \tau} d \mu \leq \alpha+\|g\|_{1} \log (\eta(\tau)+1)
$$

Seja $A=\{s:|y(s)| \geq 1\}$. Então

$$
\begin{aligned}
\int_{A} g \log y_{m, 1} d \mu & \leq \alpha+\|g\|_{L_{1}} \log (\eta(1)+1)-\int_{S \backslash A} g \log y_{m, 1} d \mu \\
& \leq \alpha+\|g\|_{L_{1}} \log (\eta(1)+1)-\int_{S \backslash A} g \log |u| d \mu \\
& \leq \alpha+\|g\|_{L_{1}} \log (\eta(1)+1)+\int g|\log u| d \mu \\
& \leq \alpha+\|g\|_{L_{1}} \log (\eta(1)+1)+\|g\|_{w^{-1}, 2} \cdot\|\log u\|_{w, 2}
\end{aligned}
$$

para todo $m \in \mathbb{N}$. Se $m \rightarrow \infty$, então pelo Teorema da Convergência Monótona obtemos que

$$
\int g \log _{+}|y| d \mu \leq \alpha+\|g\|_{L_{1}} \log (\eta(\tau)+1)+\|g\|_{w^{-1}, 2} \cdot\|\log u\|_{w, 2},
$$

para $y \in B_{X}$, consequentemente

$$
\sup _{y \in B_{X}} \int g \log _{+}|y|<\infty .
$$

Com isto, nós provamos que $g \in \mathscr{T}_{X}$.

Agora, usando a hipóteses do teorema, temos que $\int g \log |x| d \mu \leq \Phi_{X}(g)$, e como $g$ verifica $\alpha<\int g \log |x| d \mu$, segue-se que $\alpha<\Phi_{X}(g)$. 


\subsection{Propriedades dos indicadores}

Agora para $y \in B_{X}$, com cálculos similares, pode-se demonstrar que

$$
\int g \log \left(\max (|y|, \tau|u|)(\eta(\tau)+1)^{-1}\right) d \mu \leq \alpha,
$$

isto é,

$$
\int g \log (\max (|y|, \tau|u|)) d \mu \leq \alpha+\|g\|_{L_{1}} \log (\eta(\tau)+1)
$$

se $\tau \rightarrow 0$, temos que

$$
\int g \log |y| d \mu \leq \alpha, \text { para todo } y \in B_{X},
$$

portanto, $\Phi_{X}(g) \leq \alpha$, o qual é absurdo. Consequentemente $x \in B_{X}$.

Corolário 6.18 Sejam $X, Y$ espaços de funções g-convexos e $\mathscr{T}$ um semi-ideal estrito contido em $\mathscr{T}_{X} \cap \mathscr{T}_{Y}$. Então $\|x\|_{X} \leq M\|x\|_{Y}$ para todo $x \in L_{0}$ se, e somente se, $\Phi_{X}(f) \geq \Phi_{Y}(f)-$ $(\log M)\|f\|_{L_{1}}$ para todo $f \in \mathscr{T}$. Mais ainda, X e $Y$ são iguais com quase-normas equivalentes se, e somente se,

$$
d\left(\Phi_{X}, \Phi_{Y}\right)=\sup _{\|f\|_{L_{1}} \leq 1}\left|\Phi_{X}(f)-\Phi_{Y}(f)\right|<\infty .
$$

Demonstração. Assumamos primeiro que $\|x\|_{X} \leq M\|x\|_{Y}$ para todo $x \in L_{0}$. Seja $x \in B_{Y}$. Portanto, $|x| / M \in B_{X} \mathrm{e}$

$$
\begin{aligned}
\int f \log |x| d \mu-(\log M)\|f\|_{L_{1}} & =\int f \log \frac{|x|}{M} d \mu \\
& \leq \Phi_{X}(f),
\end{aligned}
$$

para todo $f \in \mathscr{T}$. Por $x$ ser arbitrário em $B_{Y}$, temos que $\Phi_{X}(f) \geq \Phi_{Y}(f)-(\log M)\|f\|_{L_{1}}$ para todo $f \in \mathscr{T}$.

Reciprocamente, assumamos que $\Phi_{X}(f) \geq \Phi_{Y}(f)-(\log M)\|f\|_{L_{1}}$ para todo $f \in \mathscr{T}$.

Seja $x \in B_{Y}$. Note que

$$
\begin{aligned}
\int f \log |x| d \mu-(\log M)\|f\|_{L_{1}} & \leq \Phi_{Y}(f)-(\log M)\|f\|_{L_{1}} \\
& \leq \Phi_{X}(f)
\end{aligned}
$$

para todo $f \in \mathscr{T}$. Isto equivale a

$$
\int f \log \frac{|x|}{M} d \mu \leq \Phi_{X}(f) \text { para todo } f \in \mathscr{T} .
$$

Pelo Teorema 6.17, temos que $\frac{x}{M} \in B_{X}$. Agora para todo $x \in Y$, obtemos que $\frac{x}{M\|x\|_{Y}} \in B_{X}$, concluindo que $\|x\|_{X} \leq M\|x\|_{Y}$ para todo $x \in L_{0}$. Note que a outra do corolário é imediata. 


\subsection{Propriedades dos indicadores}

Teorema 6.19 Seja $\mathscr{T}$ um semi-ideal estrito de $L_{1}^{+}(\mu)$ e $\Phi$ um funcional semilinear real, $K$ contínuo e convexo definido em $\mathscr{T}$. Então existe um único espaço $g$-convexo $X$ tal que $\mathscr{T} \subseteq \mathscr{T}_{X}$ $e \Phi(f)=\Phi_{X}(f)$, para todo $f \in \mathscr{T}$.

Demonstração. Vamos demonstrar o teorema por partes:

Passo 1. Vamos definir o espaço $X$.

Seja $B_{X}$ o subconjunto de $L_{0}$ de todos os $x$ tais que $f \log _{+}|x| \in L_{1}$ para todo $f \in \mathscr{T}$ e

$$
\int f \log |x| d \mu \leq \Phi(f) \text { para todo } f \in \mathscr{T} \text {. }
$$

Definamos uma função $\|\cdot\|_{X}: L_{0} \longmapsto[0, \infty]$ por

$$
\|x\|_{X}= \begin{cases}\inf \left\{\alpha>0: \alpha^{-1}|x| \in B_{X}\right\}, & \text { se } \alpha^{-1}|x| \in B_{X}, \text { para algum } \alpha>0 \\ \infty, & \text { se } \alpha^{-1}|x| \notin B_{X}, \text { para todo } \alpha>0 .\end{cases}
$$

Observe que o conjunto $B_{X}$ verifica,

$$
|y| \leq|x|, x \in B_{X} \Rightarrow y \in B_{X} .
$$

Então definimos $X$ por $X=\left\{x:\|x\|_{X}<\infty\right\}$. Note que $B_{X}=\left\{x:\|x\|_{X} \leq 1\right\}$.

Passo 2. Provaremos que $\|\lambda x\|_{X}=|\lambda| \cdot\|x\|_{X}$ para $\lambda \in \mathbb{C}$. É imediato.

Passo 3. Demonstraremos que $B_{X}$ é fechado em $L_{0}$.

Seja $\left(x_{n}\right)_{n}$ uma sequência em $B_{X}$ que converge em medida para $x$. Então , existe uma subsequência de $\left(x_{n}\right)_{n}$, que denotamos por $\left(x_{n}\right)_{n}$, tal que $\left|x_{n}\right| \rightarrow|x|$ q.s. Denotamos por $A=\operatorname{supp}(x)$. Pelo Corolário 2.62, existe uma sequência crescente de subconjuntos de $A$ mensuráveis, $\left(A_{n}\right)_{n}$, com $A_{k} \subseteq A_{k+1}$, tais que $\mu\left(A / \cup_{n} A_{n}\right)=0$, e $\left|x_{n}\right| \cdot\left|x^{-1}\right|$ converge uniformemente para 1 em $A_{n}$. Isto é, para $\varepsilon>0$ e $k \in \mathbb{N}$, existe $N \in \mathbb{N}$, tal que, para todo $s \in A_{k}$,

$$
|| x_{n}(s)|\cdot| x^{-1}(s)|-1|<\varepsilon \text { se } n \geq N .
$$

Deste fato deduzimos que

$$
(1-\varepsilon)|x| \cdot 1_{A_{k}}<\left|x_{n}\right| \cdot 1_{A_{k}} \text { se } n \geq N .
$$

Se $n \geq N$ e $f \in \mathscr{T}$, temos que, $f \log _{+}\left|x_{n}\right| 1_{A_{k}} \in L_{1}$. Isto é garantido pelo fato de $x_{n}$ ser um elemento de $B_{X}$ e pela propriedade (6.13). Agora por (6.14), obtemos que $f \log _{+}|x| 1_{A_{k}} \in L_{1}$. Consideremos $f \in \mathscr{T}$ arbitrária. Então 


\subsection{Propriedades dos indicadores}

$$
\int f \log |x| 1_{A_{k}} d \mu \leq-\log (1-\varepsilon) \cdot\|f\|_{L_{1}}+\int f \log \left|x_{n}\right| 1_{A_{k}} d \mu
$$

como $\left|x_{n}\right| 1_{A_{k}} \in B_{X}$, temos que

$$
\int f \log |x| 1_{A_{k}} d \mu \leq-\log (1-\varepsilon) \cdot\|f\|_{L_{1}}+\Phi(f)
$$

Se $\varepsilon \rightarrow 0$, obtemos que $|x| 1_{A_{k}} \in B_{X}$ para todo $k \in \mathbb{N}$. Sejam $G=\{s:|x(s)|>1\}$ e $f \in \mathscr{T}$. Observe que $0 \leq f \cdot 1_{G} \log |x| 1_{A_{k}} \leq f \cdot 1_{G} \log |x| 1_{A_{k+1}}$ para todo $k \in \mathbb{N}$, pois $A_{k} \subseteq A_{k+1}$. Também

$$
f \cdot 1_{G} \log |x| 1_{A_{k}} \rightarrow f \cdot 1_{G} \log |x| \text { q.s. em } A,
$$

pois

$$
f(s) \cdot 1_{G}(s) \log |x(s)| 1_{A_{k}}(s) \rightarrow f(s) \cdot 1_{G}(s) \log |x(s)|,
$$

para todo $s \in \cup_{n} A_{n}$, com $\mu\left(A \backslash \cup_{n} A_{n}\right)=0$. O Teorema da Convergêcia Monótona e o fato $|x| 1_{A_{k}} \in B_{X}$ implicam que

$$
\begin{aligned}
\int f \log _{+}|x| d \mu & =\int_{A} f \cdot 1_{G} \log |x| d \mu, \\
& =\lim _{k \rightarrow \infty} \int_{A} f \cdot 1_{G} \log |x| 1_{A_{k}} d \mu \\
& =\lim _{k \rightarrow \infty} \int f \cdot 1_{G} \log |x| 1_{A_{k}} d \mu \\
& \leq \Phi\left(f \cdot 1_{G}\right) .
\end{aligned}
$$

Os cálculos acima, mostram que $\int f \log _{+}|x| d \mu \in L_{1}$ para qualquer $f \in \mathscr{T}$.

Agora vejamos que $\int f \log |x| d \mu \leq \Phi(f)$, para todo $f \in \mathscr{T}$. Como $\left(x_{n}\right)_{n}$ converge para $x$ em medida, então existe uma subsequência de $\left(x_{n}\right)_{n}$, que denotamos por $\left(x_{n}\right)_{n}$, tal que $\left(x_{n}\right)_{n}$ converge q.s. para $x$.

Suponha primeiro que $f \log |x| \in L_{1}$. Seja $y_{n}=\inf _{k \geq n}\left|x_{k}\right|$. Esta sequência é crescente e $y_{n} \rightarrow|x|$ q.s., com $y_{n} \leq\left|x_{n}\right|$. Também $y_{n} \leq|x|$, portanto $f \log y_{n} \leq f \log |x|$. Pelo Teorema da Convergência Dominada, obtemos que $\int f \log y_{n} d \mu \rightarrow \int f \log |x| d \mu$, se $n \rightarrow \infty$. Como $y_{n} \in B_{X}$, pois $y_{n} \leq\left|x_{n}\right|$, onde $x_{n} \in B_{X}$, temos que $\int f \log y_{n} d \mu \leq \Phi(f)$ para todo $n \in \mathbb{N}$. Logo $\int f \log |x| d \mu \leq \Phi(f)$. Se $\int f \log |x| d \mu=-\infty$, trivialmente $-\infty=\int f \log |x| d \mu \leq \Phi(f)$. Nós provamos que $x \in B_{X}$ e portanto $B_{X}$ é fechado em $L_{0}$.

Passo 4. Demonstraremos a parte 4. da Definição 5.4.

Seja $0<\tau<\infty$ e $x, y \in B_{X}, \operatorname{com} \operatorname{supp}(x) \cap \operatorname{supp}(y)=\emptyset$. Sejam $A_{x}$ e $A_{y}$ conjuntos mensuráveis, 


\subsection{Propriedades dos indicadores}

tais que, $A_{x} \cap A_{y}=\emptyset, \operatorname{supp}(x) \subseteq A_{x}, \operatorname{supp}(y) \subseteq A_{y}$ e $A_{x} \cup A_{y}=S$. Então para para $f \in \mathscr{T}$, temos que

$$
\begin{aligned}
\int f \log \max (|x|, \tau|y|) d \mu & =\int_{A_{y}} f \log |x| d \mu+\int_{A_{x}} f \log \tau|y| d \mu \\
& \leq \Phi\left(f \cdot 1_{A_{x}}\right)+\Phi\left(f \cdot 1_{A_{y}}\right)+\left\|f \cdot 1_{A_{y}}\right\|_{L_{1}} \log \tau \\
& =\Phi(f)+\Delta_{\Phi}\left(f \cdot 1_{A_{x}}, f \cdot 1_{A_{y}}\right)+\left\|f \cdot 1_{A_{y}}\right\|_{L_{1}} \log \tau .
\end{aligned}
$$

Seja

$$
\sigma(\varepsilon)=\sup _{\|F\|_{L_{1}} \leq 1} \sup _{\|G\|_{L_{1}} \leq \varepsilon}\left|\Delta_{\Phi}(F, G)\right|
$$

Então

$$
\begin{aligned}
\int f \log \max (|x|, \tau|y|) d \mu & =\Phi(f)+\Delta_{\Phi}\left(f \cdot 1_{A_{x}}, f \cdot 1_{A_{y}}\right)+\left\|f \cdot 1_{A_{y}}\right\|_{L_{1}} \log \tau \\
& =\Phi(f)+\|f\|_{L_{1}} \cdot\left(\Delta_{\Phi}\left(\frac{f \cdot 1_{A_{x}}}{\|f\|_{L_{1}}}, \frac{f \cdot 1_{A_{y}}}{\|f\|_{L_{1}}}\right)+\frac{\left\|f \cdot 1_{A_{y}}\right\|_{L_{1}}}{\|f\|_{L_{1}}} \log \tau\right) \\
& \leq \Phi(f)+\|f\|_{L_{1}} \cdot(\sigma(\alpha)+\alpha \log \tau),
\end{aligned}
$$

onde $\alpha=\frac{\left\|f \cdot 1_{A_{y}}\right\|_{L_{1}}}{\|f\|_{L_{1}}}$. Definamos

$$
\sigma^{*}(\tau)=\sup _{0 \leq \beta \leq 1}(\sigma(\beta)+\beta \log \tau)
$$

Observe que

$$
\lim _{\tau \rightarrow 0} \sigma^{*}(\tau)=-\infty
$$

$\mathrm{e}$

$$
\int f \log \max (|x|, \tau|y|) d \mu \leq \Phi(f)+\|f\|_{L_{1}} \sigma^{*}(\tau) \Leftrightarrow \int f \log \frac{\max (|x|, \tau|y|)}{e^{\sigma^{*}(\tau)}} d \mu \leq \Phi(f),
$$

para todo $f \in \mathscr{T}$. Isto é,

$$
\frac{\max (|x|, \tau|y|)}{e^{\sigma^{*}(\tau)}} \in B_{X}
$$




\subsection{Propriedades dos indicadores}

que equivale a $\|\max (|x|, \tau|y|)\|_{X} \leq e^{\sigma^{*}(\tau)}$.

Observe que

$$
\|x+\tau y\|_{X} \leq 1+\|\max (|x|, \tau|y|)\|_{X} \leq 1+e^{\sigma^{*}(\tau)},
$$

isto é $\eta(\tau) \leq e^{\sigma^{*}(\tau)}$, demonstrando que $\lim _{\tau \rightarrow 0} \eta(\tau)=0$ e $\|x+\tau y\|_{X} \leq 1+\eta(\tau)$.

Passo 5. Este passo está dividido em três partes:

Passo 5.A Demonstremos que se $f \in \mathscr{T}$ e $\alpha$ é um número real tal que $\alpha<\Phi(f)$, então existe $x \in B_{X}$ tal que

$$
\alpha<\int f \log |x| d \mu .
$$

Passo 5.B. Demonstremos que dado $f \in \mathscr{T}$ existe $x \in B_{X}$ tal que

$$
\int f|\log | x|| d \mu<\infty
$$

Passo 5.C. O conjunto $B_{X}$ tem um elemento positivo.

AGora Provaremos o Passo 5.

Passo 5.A. Suponha que $f \in \mathscr{T}$ e $t \in \mathbb{R}$. Definamos um espaço vetorial por

$$
Y=\bigcup_{n \in \mathbb{N}} n[-f, f] .
$$

Seja $K_{t}$ o subconjunto de $Y$ de todos os $g \geq 0$ tais que $\Phi(g) \leq t$. Por $\Phi$ ser convexo, temos que $K_{t}$ é convexo. Note que $Y$ pode ser munido da norma de $L_{1}(\mu)$ e que para cada $n \in \mathbf{N}$, o conjunto $K_{t} \cap n[-f, f]$ é fechado em $\left(Y,\|\cdot\|_{L_{1}}\right)$, isto é garantido pelo Teorema 6.16. Agora definamos uma norma em $Y$ por $\|g\|_{Y}=\operatorname{ess} \sup |g(s)| f(s)^{-1}$ e seja $Y_{*}$ o predual de $\left(Y,\|\cdot\|_{Y}\right)$ (ver Definição 2.79) isto é, o conjunto de todas as funções mensuráveis $h$, com $\operatorname{supp}(h) \subseteq \operatorname{supp}(f)$ tais que $\|h\|_{Y_{*}}=\int f|h| d \mu<\infty$. Pela definição de predual, temos que $\left(Y,\|\cdot\|_{Y}\right) \cong Y_{*}^{\prime}$. O isomorfismo $\left(Y,\|\cdot\|_{Y}\right) \longrightarrow Y_{*}^{\prime}$ está dado por $g \longmapsto \varphi_{g}$, onde, $\varphi_{g}(h)=\int g h d \mu$, para todo $h \in Y_{*}$. Daqui obtemos que $Y$ pode ser munido da topologia $w^{\star}$.

Vejamos que $K_{t} \subset Y$ é $w^{\star}$-fechado em $Y$. Primeiro mostremos que $K_{t} \cap n[-f, f]$ é $w^{\star}$-fechado em $Y$. De fato, se $K_{t} \cap n[-f, f]$ é vazio, então $K_{t} \cap n[-f, f]$ é trivialmente $w^{\star}$-fechado em $Y$. Assumamos que $K_{t} \cap n[-f, f]$ é não vazio e que $\varphi_{g_{\beta}} \stackrel{w^{\star}}{\rightarrow} \varphi_{g}$, onde $g_{\beta}$ é uma rede em $K_{t} \cap n[-f, f]$, isto equivale a dizer que $\int g_{\beta} h d \mu \rightarrow \int g h d \mu$ para todo $h \in Y_{*}$. Assumamos por contradição que $g \notin K_{t} \cap n[-f, f]$. Como o conjunto $K_{t} \cap n[-f, f]$ é fechado em $\left(Y,\|\cdot\|_{L_{1}}\right)$, então existe um funcional linear contínuo $\Upsilon:\left(Y,\|\cdot\|_{L_{1}}\right) \longrightarrow \mathbb{R}$ e $a \in \mathbb{R}$ tais que

$$
\Upsilon(g)<a<\Upsilon(z) \text { para todo } z \in K_{t} \cap n[-f, f] .
$$




\subsection{Propriedades dos indicadores}

Note que o funcional $\Upsilon$ pode ser estendido, pelo teorema de Hahn-Banach, por um funcional em $L_{1}(\mu)$ de igual norma a $\Upsilon$, portanto, existe $h \in L_{\infty}$ tal que $\Upsilon(\rho)=\int \rho h d \mu$ para todo $\rho \in L_{1}(\mu)$. Portanto, a desigualdade (6.1) transforma-se em

$$
\int g h d \mu<a<\int z h d \mu \text { para todo } z \in K_{t} \cap n[-f, f],
$$

(podemos considerar $\operatorname{supp}(h) \subseteq \operatorname{supp}(f)$ e desta forma $h \in Y_{*}$ ), em particular, para $g_{\beta}$, obtemos que $\int g h d \mu<a<\int g_{\beta} h d \mu$ o qual é absurdo. Em conclusão, $g \in K_{t} \cap n[-f, f]$, portanto, para todo $n \in \mathbb{N}$ o conjunto $K_{t} \cap n[-f, f]$ é $w^{\star}$-fechado em $Y$. Agora pelo teorema de BanachDieudonné (ver Teorema 2.71), concluimos que $K_{t}$ é $w^{\star}$-fechado em $Y$.

Agora seja $f \in \mathscr{T}$ e $\alpha<\Phi(f)$ e escolhamos $\alpha_{0}$ tal que $\alpha<\alpha_{0}<\Phi(f)$. Definamos um subconjunto de $Y \times \mathbb{R}$ por

$$
C=\left\{(g, t) \in Y^{+} \times \mathbb{R}: \Phi(g) \leq t\right\} .
$$

Similarmente, $Y \times \mathbb{R}$ pode ser munido da topologia $w^{\star}$, isto é, $Y \times \mathbb{R} \cong(Y \times \mathbb{R})_{*}^{\prime} \cong\left(Y_{*} \times \mathbb{R}\right)^{\prime}$, onde o isomorfismo está dado por $(g, t) \longmapsto\left\langle\varphi_{g}, t\right\rangle$, com, $\left\langle\varphi_{g}, t\right\rangle(h, a)=\varphi_{g}(h)+a t$, para todo $(h, a) \in Y_{*} \times \mathbb{R}$. Por $\Phi$ ser convexo, temos que $C$ é um conjunto convexo. O fato de $K_{t}$ ser $w^{\star}$-fechado em $Y$ para todo $t \in \mathbb{R}$, implica que $C$ é $w^{\star}$-fechado em $Y \times \mathbb{R}$. Abusando da notação , $\left\langle\varphi_{f}, \alpha\right\rangle \notin C$, então o Teorema 2.72 implica que existe $\left(h_{0}, a\right) \in Y_{*} \times \mathbb{R}$ tal que

$$
\left\langle\varphi_{f}, \alpha\right\rangle\left(h_{0}, a\right)<\left\langle\varphi_{g}, t\right\rangle\left(h_{0}, a\right) \text { para todo }(g, t) \in C,
$$

isto é,

$$
\varphi_{f}(h)+\alpha a<\varphi_{g}\left(h_{0}\right)+t a \text { para todo }(g, t) \in C,
$$

Observe que se $(g, t) \in C$, então $\left(\frac{g}{n}, \frac{t}{n}\right) \in C$ para todo $n \in \mathbb{N}$, deduzindo pela última desiguldade que $\varphi_{f}\left(h_{0}\right)+a \alpha \leq 0$. Note também que se $(g, t) \in C$, então $(n g, n t) \in C$ para todo $n \in \mathbb{N}$, assim $0 \leq \varphi_{g}\left(h_{0}\right)+$ at para todo $(g, t) \in C$. Resumindo, demonstramos que

$$
\varphi_{f}\left(h_{0}\right)+a \alpha \leq 0
$$

$\mathrm{e}$

$$
0 \leq \varphi_{g}\left(h_{0}\right)+\text { at para todo }(g, t) \in C .
$$

Se $t \rightarrow \infty$ na desigualdade (6.17) obtemos que $a \geq 0$. Agora demonstraremos que $a>0$. Assumamos por contradição que $a=0$. Então (6.16) e (6.17) transformam-se respectivamente em $\varphi_{f}\left(h_{0}\right) \leq 0$ e $0 \leq \varphi_{g}\left(h_{0}\right)$ para todo $g \in Y^{+}$. Note que para qualquer $\rho \in Y$, encontramos $n \in \mathbb{N}$ tal que $-n f \leq \rho \leq n f$, isto é, $\rho+n f \in Y^{+}$. Agora $\varphi_{\rho}\left(h_{0}\right)=\varphi_{\rho+n f}\left(h_{0}\right)-n \varphi_{f}\left(h_{0}\right) \geq 0$ para todo $\rho \in Y$ e em consequência temos que $\varphi_{\rho}\left(h_{0}\right)=0$, para todo $\rho \in Y$. Isto mostra que $\left(h_{0}, a\right)=(0,0)$ o qual é absurdo, portanto $a>0$.

Definamos um funcional linear contínuo $\beta$ em $Y$ por

$$
\beta(g)=\frac{-\varphi_{g}\left(h_{0}\right)}{a}=\int g \cdot \frac{-h_{0}}{a} d \mu \text {. para todo } g \in Y .
$$




\subsection{Propriedades dos indicadores}

De (6.16) e (6.17), deduzimos respectivamente que $\alpha_{0} \leq \beta(f)$ e $\beta(g) \leq t$ para todo $(g, t) \in C$. Note que para $g \in Y^{+}$temos que $(g, \Phi(g)+\varepsilon) \in C$ para todo $\varepsilon>0$, portanto, $\beta(g) \leq \Phi(g)+\varepsilon$ para todo $\varepsilon>0$, isto é, $\beta(g) \leq \Phi(g)$ para todo $g \in Y^{+}$. Até o momento, provamos que existe um funcional linear contínuo $\beta: Y \rightarrow \mathbb{R}$ tal que $\alpha<\alpha_{0} \leq \beta(f)$ e $\beta(g) \leq \Phi(g)$ para todo $g \in Y^{+}$, isto é, $\alpha<\int f h d \mu$ e $\int g h d \mu \leq \Phi(g)$ para todo $g \in Y^{+}$, onde $h=\frac{-h_{0}}{a}$.

Definamos uma função $x$ de $L_{0}$ por

$$
x(s)=\left\{\begin{array}{ll}
e^{h(s)} & \text { se } s \in \operatorname{supp}(f), \\
0, & \text { se } s \notin \operatorname{supp}(f)
\end{array} .\right.
$$

Demonstremos que $x \in B_{X}$. De fato, seja $g \in \mathscr{T}$ e denotemos $A=\{s: h(s)>0\}$. Então pelo fato de $\min \left(g \cdot 1_{A}, n f\right) \in Y^{+}$e pelo Teorema 6.16, temos que

$$
\begin{aligned}
\int g \log _{+}|x| d \mu & =\int g h d \mu \\
& =\lim _{n \rightarrow \infty} \int_{A} \min (g, n f) h d \mu \\
& \leq \liminf _{n \rightarrow \infty} \Phi\left(\min \left(g \cdot 1_{A}, n f\right)\right) \\
& =\Phi\left(g \cdot 1_{A}\right)
\end{aligned}
$$

$\log 0 \log _{+}|x| \in L_{1}$.

Agora provaremos que $\int g \log |x| d \mu \leq \Phi(g)$. De fato, se $\operatorname{supp}(g) \nsubseteq \operatorname{supp}(f)$, então existe um conjunto de medida positiva $E \subset \operatorname{supp}(g)$, tal que $x(s)=0$ para todo $s \in E$, portanto $\int g \log |x| d \mu=-\infty$ e imediatamente demonstramos que $\int g \log |x| d \mu \leq \Phi(g)$.

Agora suponha que $\operatorname{supp}(g) \subseteq \operatorname{supp}(f)$. Vejamos que $g \log |x| \in L_{1}$ e $\int g \log |x| d \mu \leq \Phi(g)$. De fato, considere a sequência $g_{n}=\min (g, n f)$. Note que $g_{n} \rightarrow g \cdot 1_{\text {supp }(f)}$ q.s. e $g \cdot 1_{\text {supp }(f)}=g$ pelo fato $\operatorname{supp}(g) \subseteq \operatorname{supp}(f)$. Devido a que $\left|h g_{n}\right| \leq n f|h| \in L_{1}$, temos pelo Teorema da Convergência Dominada que $g \log |x| \in L_{1} \mathrm{e}$

$$
\begin{aligned}
\int g \log |x| d \mu & =\int_{\operatorname{supp}(g)} g h d \mu \\
& =\lim _{n \rightarrow \infty} \int g_{n} h d \mu .
\end{aligned}
$$




\subsection{Propriedades dos indicadores}

Agora segue da igualdade acima, o Teorema 6.16 e pelo fato de $\min (g, n f) \in Y^{+}$, que

$$
\begin{aligned}
\int g \log |x| d \mu & \leq \liminf _{n \rightarrow \infty} \Phi(\min (g, n f)), \\
& =\Phi(g)
\end{aligned}
$$

isto mostra que $x \in B_{X}$. Observe que $\int f \log |x| d \mu=\int f h d \mu>\alpha$, daqui mostramos que existe $x \in B_{X}$ tal que $\alpha<\int f \log |x| d \mu$.

Passo 5.B. É imediato, pois $\int f|\log | x|| d \mu=\int f|h| d \mu<\infty$.

Passo 5.C. Pelo fato de $\mathscr{T}$ ser um semi-ideal estrito, temos que existe $f \in \mathscr{T}$, com $f>0$. Portanto $x(s)=e^{h(s)}>0$ para todo $s \in \operatorname{supp}(f)=S$.

Os Passos 2,3,4 e 5.C. mostram que $X$ é um espaço de funções $g$-convexo.

Passo 6. Vejamos que $\mathscr{T} \subseteq \mathscr{T}_{X}$ e

$$
\Phi(f)=\Phi_{X}(f)=\sup _{x \in B_{X}} \int f \log |x| d \mu .
$$

De fato, seja $f \in \mathscr{T}$ e $x \in B_{X}$. Então denotando por $A=\{x:|x|>1\}$, segue do Teorema ??

$$
\begin{aligned}
\int f \log _{+}|x| d \mu & =\int 1_{A} f \cdot \log |x| d \mu \\
& \leq \Phi\left(1_{A} \cdot f\right) \\
& \leq \sup _{g \in[0, f]} \Phi(g),
\end{aligned}
$$

$\log 0 \sup _{x \in B_{X}} \int f \log _{+}|x| d \mu<\infty$.

No Passo 5.B., nós demonstramos que dado $f \in \mathscr{T}$, existe $x \in B_{X}$ tal que

$$
\int f|\log | x|| d \mu=\int f|h| d \mu<\infty,
$$

portanto $f \in \mathscr{T}_{X}$, e consequentemente $\mathscr{T} \subseteq \mathscr{T}_{X}$.

Agora no Passo 5.A. nós demostramos que dado $\alpha$ tal que $\alpha<\Phi(f)$ existe $x \in B_{X}$ tal que $\alpha<\int f \log |x| d \mu \leq \Phi(f)$, isto prova imediatamente que

$$
\Phi(f)=\Phi_{X}(f)=\sup _{x \in B_{X}} \int f \log |x| d \mu .
$$




\subsection{Propriedades dos indicadores}

Passo 7. Demonstraremos a unicidade do teorema. Sejam $X$ e $Y$ espacos $g$-convexos tais que $\Phi=\Phi_{X}=\Phi_{Y}$. Vejamos que $X=Y$.

Seja $x \in B_{X}$. Então $f \log _{+}|x| \in L_{1}$ e $\int f \log |x| d \mu \leq \Phi_{X}(f)=\Phi_{Y}(f)$ para todo $f \in \mathscr{T}$. Pelo Teorema 6.17 temos que $x \in B_{Y}$. Logo $B_{X} \subseteq B_{Y}$. Similarmente mostramos que $B_{Y} \subseteq B_{X}$, e portanto $B_{X}=B_{Y}$, concluindo que $X=Y$.

Teorema 6.20 Seja $\mathscr{T}$ um semi-ideal estrito e $\Phi: \mathscr{T} \longrightarrow \mathbb{R}$ um funcional semi-linear. Então para que exista um único espaço de Köthe $X$, com $\mathscr{T} \subseteq \mathscr{T}_{X}$ e $\Phi(f)=\Phi_{X}(f)$ para $f \in \mathscr{T}$,é necessário e suficiente que $\Phi$ e $\Lambda-\Phi$ sejam convexos.

Demonstração. Se existe um espaço de Köthe $X$, tal que $\mathscr{T} \subseteq \mathscr{T}_{X}$ e $\Phi(f)=\Phi_{X}(f)$ para $f \in \mathscr{T}$, obtemos imediatamente que $\Phi$ e $\Lambda-\Phi$ são convexos, pois no Teorema 6.15 mostramos que $\Phi_{X}=\Phi$ e $\Lambda-\Phi=\Lambda-\Phi_{X}=\Phi_{X^{*}}$ são convexos.

Reciprocamente, suponha que $\Phi$ e $\Lambda-\Phi$ são convexos. Isto implica que

$$
0 \leq \Delta_{\Phi}(f, g) \leq \Delta_{\Lambda}(f, g)
$$

portanto,

$$
0 \leq \sup _{\|f\|_{L_{1}} \leq 1} \sup _{\|g\|_{L_{1}} \leq \varepsilon} \Delta_{\Phi}(f, g) \leq \sup _{\|f\|_{L_{1}} \leq 1} \sup _{\|g\|_{L_{1}} \leq \varepsilon}\left|\Delta_{\Lambda}(f, g)\right|
$$

como $\Lambda$ é $K$ - contínuo (ver Teorema 6.15), temos que

$$
\lim _{\varepsilon \rightarrow 0} \sup _{\|f\|_{L_{1}} \leq 1} \sup _{\|g\|_{L_{1}} \leq \varepsilon}\left|\Delta_{\Phi}(f, g)\right|=0 .
$$

Isto prova que que $\Phi$ é $K$-contínuo, portanto, $\Lambda-\Phi$ é também $K$-contínuo. O Teorema 6.19 garante a existência de dois espaços $g$-convexos $X$ e $Y$ tais que $\mathscr{T} \subseteq \mathscr{T}_{X} \cap \mathscr{T}_{Y}$ e $\Phi=\Phi_{X}, \Lambda-\Phi=$ $\Phi_{Y}$ em $\mathscr{T}$. Demonstraremos que $X$ é um espaço de Köthe. De fato, definamos o conjunto $B_{\widetilde{X}}$ como $B_{\widetilde{X}}=\overline{C\left(B_{X}\right)}$ (Fecho na topologia em medida da envoltória convexa da bola unitária de $X)$. Definamos agora uma função $\|\cdot\|_{\tilde{X}}: L_{0} \longmapsto[0, \infty]$ como

$$
\|\tilde{x}\|_{\widetilde{X}}= \begin{cases}\inf \left\{\alpha>0: \alpha^{-1}|\tilde{x}| \in B_{\widetilde{X}}\right\}, & \text { se } \alpha^{-1}|\tilde{x}| \in B_{\widetilde{X}}, \text { para algum } \alpha>0 \\ \infty, & \text { se } \alpha^{-1}|\tilde{x}| \notin B_{\widetilde{X}}, \text { para todo } \alpha>0 .\end{cases}
$$

Definamos $\widetilde{X}=\left\{\tilde{x}:\|\tilde{x}\|_{\widetilde{X}}<\infty\right\}$. Demonstraremos que $\widetilde{X}$ é um espaço de Köthe e $\widetilde{X}=X$. De fato, observe que $\|\cdot\|_{\tilde{X}}$ foi definido como o funcional de Minkowski em $B_{\widetilde{X}}$. Agora $B_{\widetilde{X}}$ é convexo, balançado e absorvente, portanto $\|\cdot\|_{\tilde{X}}$ é uma semi-norma (ver Proposição 2.75). Se $D=\left\{\tilde{x} \in \widetilde{X}:\|\tilde{x}\|_{\widetilde{X}} \leq 1\right\}$, então pelo fato de ser $B_{\widetilde{X}}$ fechado na topologia em medida e pela forma como definimos $\|\cdot\|_{\tilde{X}}$, temos que $B_{\widetilde{X}}=D$. Com este fato nós mostramos que $\|\tilde{x}\|_{\tilde{X}}=0$, implica $\tilde{x}=0$. Logo, $\|\cdot\|_{\tilde{X}}$ é uma norma. É imediato ver que $B_{\widetilde{X}}$ é fechado em $L_{0}$. Note que $B_{\widetilde{X}}$ 


\subsection{Propriedades dos indicadores}

contém um elemento positivo, pois $B_{X} \subseteq B_{\widetilde{X}}$, onde $B_{X}$ tem um elemento positivo. É imediato verificar que se $|\tilde{y}| \leq|\tilde{x}|$, $\operatorname{com} \tilde{x} \in \tilde{X}$, então $\tilde{y}$ e $\|\tilde{y}\|_{\tilde{X}} \leq\|\tilde{x}\|_{\tilde{X}}$. Por enquanto, nós provamos que $\widetilde{X}$ é um espaço de funções $g$-convexo. Para demonstrar que $\widetilde{X}$ é um espaço de Köthe, basta verificar que existe $h>0$ tal que $\|h \tilde{x}\|_{L_{1}} \leq\|\tilde{x}\|_{\widetilde{X}}$ para todo $\tilde{x} \in \widetilde{X}$. Se $B_{\widetilde{X}} \cdot B_{Y} \subseteq B_{L_{1}}$ é válido, então imediatamente $\widetilde{X}$ é um espaço de Köthe, pois escolhendo um elemento positivo $h=y \mathrm{em} B_{Y}$ (isto é possível porque $Y$ é um espaço de funções $g$-convexo), obtemos que

$$
\|\tilde{x} \cdot h\|_{L_{1}}=\left\|\frac{\tilde{x}}{\|\tilde{x}\|_{\tilde{X}}} \cdot h\right\|_{L_{1}} \cdot\|\tilde{x}\|_{\tilde{X}} \leq 1 \cdot\|\tilde{x}\|_{\tilde{X}}
$$

para todo $\tilde{x} \in \tilde{X}$.

Afirmamos que $B_{\widetilde{X}} \cdot B_{Y} \subseteq B_{L_{1}}$. Provaremos primeiro que $B_{X} \cdot B_{Y} \subseteq B_{L_{1}}$. De fato, sejam $x \in$ $B_{X}$ e $y \in B_{Y}$. Então para todo $f \in \mathscr{T} \subseteq \mathscr{T}_{X} \cap \mathscr{T}_{Y}$ temos que $f \log _{+}|x|, f \log _{+}|y| \in L_{1}$, isto segue da definição dos semi-ideais $\mathscr{T}_{X}, \mathscr{T}_{Y}$. Pelas propriedades do logaritmo e pelo fato anterior mostramos que $f \log _{+}|x y| \in L_{1}$ para todo $f \in \mathscr{T}$. Agora para $f \in \mathscr{T}$ temos que

$$
\begin{aligned}
\int f \log |x y| d \mu & =\int f \log |x| d \mu+\int f \log |y| d \mu \\
& \leq \Phi_{X}(f)+\Phi_{Y}(f) \\
& =\Lambda(f) .
\end{aligned}
$$

Resumindo temos que $\int f \log _{+}|x y| \in L_{1}$ e $\int f \log |x y| d \mu \leq \Lambda(f)$ para todo $f \in \mathscr{T}$. Então o Teorema 6.17 garante que $x y \in B_{L_{1}}$. Agora, vejamos que $B_{X} \cdot B_{Y} \subseteq B_{L_{1}}$ implica $B_{\widetilde{X}} \cdot B_{Y} \subseteq B_{L_{1}}$. De fato:

Sejam $x \in B_{\widetilde{X}}$ e $y \in B_{Y}$. Se $x \in B_{X}$, então temos que $x y \in B_{L_{1}}$. Se $x \in C\left(B_{X}\right)$, então $x=\sum_{i=1}^{n} \lambda_{i} x_{i}$, onde $\lambda_{i} \geq 0, \sum_{i=1}^{n} \lambda_{i}=1$ e $x_{i} \in B_{X}$. Agora como $x_{i} y \in B_{X} \cdot B_{Y}$ temos que $x_{i} y \in B_{L_{1}}$, portanto $x y \in B_{L_{1}}$.

Se $x \in \overline{C(X)}$, então existe uma sequência $\left(x_{n}\right)_{n}$ de elementos em $C\left(B_{X}\right)$, tais que, $x_{n} \longrightarrow x$ q.s.,isto implica que $x_{n} y \longrightarrow x y$ q.s. Como $y \in B_{X}$, temos que $x_{n} y \in B_{L_{1}}$, portanto, $x_{n} y$ converge em medida para $x y$. Pelo Teorema 2.63 obtemos que $x y \in B_{L_{1}}$. Logo, concluimos que $B_{\widetilde{X}} \cdot B_{Y} \subseteq B_{L_{1}}$.

Demonstraremos que $X=\widetilde{X}$, para isso basta provar que $\Phi_{X}=\Phi_{\widetilde{X}}$, pois o Teorema 6.19 garante a unicidade dos espaços. De fato, devido que $B_{X} \subseteq B_{\widetilde{X}}$ temos que

$$
\Phi_{X}(f)=\sup _{x \in B_{X}} \int f \log |x| d \mu \leq \sup _{x \in B_{\widetilde{X}}} \int f \log |\tilde{x}| d \mu=\Phi_{\widetilde{X}}(f),
$$

para todo $f \in \mathscr{T} \cap \mathscr{T}_{\bar{X}} \operatorname{Logo}, \Phi_{X}(f) \leq \Phi_{\widetilde{X}}(f)$ para todo $f \in \mathscr{T} \cap \mathscr{T}_{\bar{X}}$.

Afirmamos que $\Phi_{\widetilde{X}}(f) \leq \Lambda(f)-\Phi_{Y}(f)$ para todo $f \in \mathscr{T} \cap \mathscr{T}_{\widetilde{X}}$. De fato, usando $B_{\widetilde{X}} \cdot B_{Y} \subseteq B_{L_{1}}$, deduzimos que 


$$
\int f \log |\tilde{x} y| d \mu \leq \sup _{z \in B_{L_{1}}} \int f \log |z| d \mu=\Lambda(f),
$$

para todo $f \in \mathscr{T} \cap \mathscr{T}_{\widetilde{X}}, \tilde{x} \in B_{\widetilde{X}}$ e $y \in B_{Y}$. Isto equivale a

$$
\int f \log |\tilde{x}| d \mu+\int f \log |y| d \mu \leq \Lambda(f)
$$

para todo $\tilde{x} \in B_{\widetilde{X}}$ e todo $y \in B_{Y}$, portanto $\Phi_{\widetilde{X}}(f) \leq \Lambda(f)-\Phi_{Y}(f)=\Phi_{X}(f)$ para todo $f \in \mathscr{T} \cap \mathscr{T}_{\widetilde{X}}$. Consequentemente, $\Phi_{X} \leq \Phi_{\widetilde{X}} \leq \Phi_{X}$ em $\mathscr{T} \cap \mathscr{T}_{\widetilde{X}}$, logo $\Phi_{X}=\Phi_{\widetilde{X}}$, e portanto $X=\widetilde{X}$.

\subsection{Aproximação de funcionais semilineares}

Nesta seção vamos aproximar funcionais semilineares por meio de funções indicadoras de espaços de Köthe.

Proposição 6.21 Seja $\mathscr{T}$ um semi-ideal estrito contido em $(L \log L)_{+} e \Phi$ um funcional semilinear em $\mathscr{T}$. Suponha que para algum $M$ e todo $f_{1}, \ldots, f_{n} \in \mathscr{T}$, temos que

$$
-M \sum_{k=1}^{n}\left\|f_{k}\right\|_{L_{1}} \leq \Delta_{\Phi}\left(f_{1}, \ldots, f_{n}\right) \leq \Delta_{\Lambda}\left(f_{1}, \ldots, f_{n}\right)+M \sum_{k=1}^{n}\left\|f_{k}\right\|_{L_{1}} .
$$

Então, existe um espaço de Köthe $X$ tal que $\mathscr{T} \subset \mathscr{T}_{X} e$

$$
d\left(\Phi, \Phi_{X}\right)=\sup _{\|f\|_{L_{1}} \leq 1}\left|\Phi(f)-\Phi_{X}(f)\right| \leq M+4 \delta,
$$

onde $\delta=\delta(\Phi)$.

Demonstração.

Caso 1. Primeiro assumamos que $S$ seja um conjunto finito e $\mathscr{T}=L_{1}^{+}(S)$. Definamos $\Phi: \mathscr{T} \rightarrow$ $\mathbb{R}$ por $\Phi_{0}(f)=\inf \sum_{j=1}^{n} \Phi\left(f_{j}\right)$, o ínfimo é tomado no conjuntos dos $f_{j}$ tais que $f=\sum_{j=1}^{n} f_{j}$, com $f_{j} \geq 0$. O funcional $\Phi_{0}$ assim definido é convexo, pois se $f=\sum f_{s}$ e $g=\sum g_{k}$, então $\Phi(f+g) \leq \sum_{k} \Phi\left(f_{k}\right)+\sum \Phi\left(g_{s}\right)$, concluindo que $\Phi(f+g) \leq \Phi(f)+\Phi(g)$. Note que se $f=\sum_{j=1}^{n} f_{j}$. Usando a hipóteses do teorema, provamos que

$$
\begin{aligned}
\Phi(f)-M\|f\|_{L_{1}} & =\Phi\left(\sum_{j=1}^{n} f_{j}\right)-M\|f\|_{L_{1}} \\
& =\sum_{j=1}^{n} \Phi\left(f_{j}\right)-\Delta_{\Phi}\left(f_{1}, \ldots, f_{n}\right)-M\|f\|_{L_{1}} \\
& \leq \sum_{j=1}^{n} \Phi\left(f_{j}\right)
\end{aligned}
$$




\subsection{Aproximação de funcionais semilineares}

para todo $f=\sum_{j=1}^{n} f_{j}$. Portanto

$$
\Phi(f)-M\|f\|_{L_{1}} \leq \Phi_{0}(f) \leq \Phi(f) .
$$

Seja $P=\left\{x \in L_{1}(\mu): x(s)>0\right.$, para todo $\left.s \in S\right\}$. Por $L_{1}(\mu)$ ser um espaço de dimensão finita, podemos munir a $P$ da norma do máximo $\|\cdot\|_{\infty}$.

Verifiquemos agora que existe uma constante $K$ tal que

$$
\left|\Phi_{0}(f)\right| \leq K \text { para todo } f \in P, \operatorname{com}\|f\|_{\infty} \leq 1 \text {. }
$$

De fato, escrevamos $S=\cup_{k=1}^{n} A_{k}$, onde os $A_{k}$ são disjuntos e $\mu\left(A_{k}\right)<\infty$ (lembre-se que $\mu$ é $\sigma$-finita e $S$ é finito). Note que se $f \in P$, com $\|f\|_{\infty} \leq 1$, então $f=\sum_{k=1}^{n} c_{k} \cdot 1_{A_{k}}$, para alguns $0<c_{k} \leq 1$. Agora pela desigualdade (6.18) e pela Observação 6.12 segue-se que

$$
\begin{aligned}
\left|\Phi_{0}(f)\right| & \leq\left|\Phi_{0}\left(\sum_{k=1}^{n} c_{k} \cdot 1_{A_{k}}\right)-\Phi\left(\sum_{k=1}^{m} c_{k} \cdot 1_{A_{k}}\right)\right|+\left|\Phi\left(\sum_{k=1}^{n} c_{k} \cdot 1_{A_{k}}\right)\right| \\
& \leq M \sum_{k=1}^{n}\left\|c_{k} \cdot 1_{A_{k}}\right\|_{L_{1}}+\left|\Phi\left(\sum_{k=1}^{n} c_{k} \cdot 1_{A_{k}}\right)\right| \\
& =M \sum_{k=1}^{n}\left\|c_{k} \cdot 1_{A_{k}}\right\|_{L_{1}}+\left|\sum_{k=1}^{n} c_{k} \Phi\left(1_{A_{k}}\right)-\Delta_{\Phi}\left(c_{1} \cdot 1_{A_{1}}, \ldots, c_{m} \cdot 1_{A_{n}}\right)\right| \\
& \leq M \mu(S)+\sum_{k=1}^{n}\left|\Phi\left(1_{A_{k}}\right)\right|+\left|\Delta_{\Phi}\left(c_{1} \cdot 1_{A_{1}}, \ldots, c_{m} \cdot 1_{A_{n}}\right)\right|, \\
& \leq M \mu(S)+\sum_{k=1}^{n}\left|\Phi\left(1_{A_{k}}\right)\right|+(n-1) \delta(\Phi) \sum_{k=1}^{n}\left\|c_{k} \cdot 1_{A_{k}}\right\|_{L_{1}} \\
& \leq M \mu(S)+\sum_{k=1}^{n}\left|\Phi\left(1_{A_{k}}\right)\right|+(n-1) \delta(\Phi) \mu(S) .
\end{aligned}
$$

Agora demonstraremos que $\Phi_{0}$ é contínua em $\left(P,\|\cdot\|_{\infty}\right)$. De fato, seja $f_{n} \rightarrow f$, onde $\left(f_{n}\right)_{n}$ e $f$ pertence a $P$, reescrevendo, $\frac{f_{n}}{f} \rightarrow 1$. Então para $\varepsilon>0$, existe $N \in \mathbb{N}$, tal que

$$
(1-\varepsilon) f \leq f_{n} \leq(1+\varepsilon) f
$$

Agora, para $s \in S$ e $n \geq \mathbb{N}$, temos por (6.19) 


\subsection{Aproximação de funcionais semilineares}

$$
\begin{aligned}
0 \leq f_{n}(s)-(1-\varepsilon) f(s) \leq 2 \varepsilon f(s) & \Leftrightarrow(1-\varepsilon) f(s) \leq f_{n}(s) \leq(1+\varepsilon) f(s) \leq 2 \varepsilon\|f\|_{\infty} \\
& \Rightarrow \frac{f_{n}-(1-\varepsilon) f}{2 \varepsilon\|f\|_{\infty}} \leq 1 \\
& \Leftrightarrow\left\|\frac{f_{n}-(1-\varepsilon) f}{2 \varepsilon\|f\|_{\infty}}\right\|_{\infty} \leq 1 \\
& \Rightarrow\left|\Phi_{0}\left(f_{n}-(1-\varepsilon) f\right)\right| \leq 2 \varepsilon K\|f\|_{\infty} .
\end{aligned}
$$

Similarmente demonstramos que

$$
\left|\Phi_{0}\left((1+\varepsilon) f-f_{n}\right)\right| \leq 2 \varepsilon\|f\|_{\infty} K
$$

sempre que $n \geq \mathbb{N}$. Agora pelo fato de $\Phi_{0}$ ser convexo, temos que

$$
\begin{aligned}
\Phi_{0}\left(f_{n}\right)-(1-\varepsilon) \Phi_{0}(f) & =\Phi_{0}\left(f_{n}-(1-\varepsilon) f+(1-\varepsilon) f\right)-\Phi_{0}(f(1-\varepsilon)) \\
& \leq \Phi_{0}\left(f_{n}-(1-\varepsilon) f\right) \\
& \leq 2 \varepsilon K\|f\|_{\infty}
\end{aligned}
$$

portanto,

$$
\Phi_{0}\left(f_{n}\right) \leq(1-\varepsilon) \Phi_{0}(f)+2 \varepsilon K\|f\|_{\infty}, \text { se } n \geq \mathbb{N}
$$

Analogamente demonstramos que

$$
(1+\varepsilon) \Phi_{0}(f) \leq \Phi_{0}\left(f_{n}\right)+2 \varepsilon K\|f\|_{\infty}, \text { se } n \geq \mathbb{N} .
$$

Pelas últimas desigualdade, concluimos que

$$
\left|\Phi_{0}(f)-\Phi_{0}\left(f_{n}\right)\right| \leq \varepsilon\left|\Phi_{0}(f)\right|+2 \varepsilon K\|f\|_{\infty} \text { se } n \geq \mathbb{N} .
$$

Daqui, $\lim _{n \rightarrow \infty} \Phi\left(f_{n}\right)=\Phi(f)$ e assim $\Phi$ é contínua em $\left(P,\|\cdot\|_{\infty}\right)$, portanto, contínua em $\left(P,\|\cdot\|_{L_{1}}\right)$ por $L_{1}(\mu)$ ser de dimensão finita.

Definamos o conjunto $B_{0}$ de todas as funções $x \in L_{0}(\mu)$, tais que

$$
\int f \log |x| d \mu \leq \Phi_{0}(f), \quad \forall f \in P
$$


Vejamos que

$$
\Phi_{0}(f)=\sup _{x \in B_{0}} \int f \log |x| d \mu .
$$

Fixemos $f \in P$. Demonstraremos que existe um funcional linear $\bar{\beta}: L_{1}(\mu) \longrightarrow \mathbb{R}$ tal que $\Phi_{0}(f) \leq \bar{\beta}(f)$ e $\bar{\beta}(g) \leq \Phi_{0}(g)$ para todo $g \in P$. De fato, seja $W=\{\lambda f: \lambda>0\}$.

Definamos uma função $\beta: W \longrightarrow \mathbb{R}$ por $\beta(\lambda f)=\lambda \Phi_{0}(f)$. Note que $\beta(g) \leq \Phi_{0}(g)$ para todo $g \in W$.

Definamos dois subconjuntos não vazios de $L_{1}(\mu) \times \mathbb{R}$ por

$$
C=\left\{(g, t) \in P \times \mathbb{R}: \Phi_{0}(g)<t\right\},
$$

e,

$$
\Gamma=\{(g, \beta(g)): g \in W\} .
$$

Pelo fato de $\Phi_{0}$ ser contínua e convexa, temos que $C$ é um subconjunto aberto e convexo de $L_{1}(\mu) \times \mathbb{R}$. É imediato verificar que $\Gamma$ é um subconjunto convexo de $L_{1}(\mu) \times \mathbb{R}$. Da desigualdade $\beta(g) \leq \Phi_{0}(g)$ para todo $g \in W$, deduzimos que $C$ e $\Gamma$ são conjuntos disjuntos. Então $C$ e $\Gamma$ verificam as hipóteses do teorema de Hahn-Banach geométrico (ver Teorema 2.65), portanto, existe um funcional linear $\varphi: L_{1}(\mu) \times \mathbb{R} \rightarrow \mathbb{R}$ tal que

$$
\varphi(h, \beta(h))<\varphi(g, \tau), \quad \forall h \in W e \forall(g, \tau) \in C .
$$

Escrevendo $\varphi(x, t)=l(x)+$ at, para todo $x \in L_{1}(\mu)$ e todo $t \in \mathbb{R}$, onde $l: L_{1}(\mu) \longrightarrow \mathbb{R}$ é um funcional linear e $a \in \mathbb{R}$, temos

$$
l(h)+a \beta(h)<l(g)+a \tau, \quad \forall h \in W e \forall(g, \tau) \in C,
$$

isto é,

$$
\lambda l(f)+\lambda a \beta(f)<l(g)+a \tau, \quad \forall \lambda>0 e \forall(g, \tau) \in C,
$$

assim, $l(f)+a \beta(f) \leq 0$, portanto $l(h)+a \beta(h) \leq 0$ para todo $h \in W$. Note que se $(g, \tau) \in C$, então $(n g, n \tau) \in C$ para todo $n \in \mathbb{N}$. Portanto $0 \leq l(g)+a \tau, \forall(g, \tau) \in C$. Resumindo, demonstramos que

$$
l(h)+a \beta(h) \leq 0, \quad \forall h \in W
$$

$\mathrm{e}$

$$
0 \leq l(g)+a \tau, \quad \forall(g, \tau) \in C .
$$

Se $\tau \rightarrow \infty$ na desigualdade (6.21) obtemos que $a \geq 0$. Demonstraremos que $a>0$. Assumamos por contradição que $a=0$. Então (6.20) e (6.21) transformam-se respectivamente em $l(h) \leq 0$ para todo $h \in W$ e $0 \leq l(g)$ para todo $g \in P$. Portanto $l(g-h) \geq 0$ para todo $g \in P$ e todo $h \in W$. Note que para $x \in L_{1}(\mu)$, por $\operatorname{Im}(x)$ ser um conjunto finito, encontramos $\lambda>0$ tal que $x+\lambda f>0$, isto é, $x+\lambda f \in P$. Agora $l(x)=l(x+\lambda f-\lambda f) \geq 0$ para todo $x \in L_{1}(\mu)$. Logo $l=0$. 


\subsection{Aproximação de funcionais semilineares}

Isto mostra que $\varphi=0$ o qual é absurdo. Consequentemente $a>0$.

Definamos um funcional linear $\bar{\beta}$ em $L_{1}(\mu)$ por

$$
\bar{\beta}=\frac{-l}{a} .
$$

De (6.20) e (6.21) deduzimos que $\beta(h) \leq \bar{\beta}(h)$, para todo $h \in W$, em particular $\Phi_{0}(f) \leq \bar{\beta}(f)$, e $\bar{\beta}(g) \leq \tau$ para todo $(g, \tau) \in C$. Note que para $g \in P$, temos que $\left(g, \Phi_{0}(g)+\varepsilon\right) \in C$ para todo $\varepsilon>0$. Portanto $\bar{\beta}(g) \leq \Phi_{0}(g)+\varepsilon$ para todo $\varepsilon>0$, isto é, $\bar{\beta}(g) \leq \Phi_{0}(g)$ para todo $g \in P$. Consequentemente, existe um funcional linear $\bar{\beta}: L_{1}(\mu) \rightarrow \mathbb{R}$ tal que

$$
\Phi_{0}(f) \leq \bar{\beta}(f) \text { e } \bar{\beta}(g) \leq \Phi_{0}(g), \quad \forall g \in P .
$$

Provaremos que dado $\alpha$ tal que $\alpha<\Phi_{0}(f)$, existe $x_{0} \in B_{0}$ que verifica $\alpha<\int f \log \left|x_{0}\right| d \mu$. De fato, pelo anterior existe um funcional linear $\bar{\beta}: L_{1}(\mu) \rightarrow \mathbb{R}$ que verifica (6.22). Nós podemos escrever o funcional $\bar{\beta}$ como $\bar{\beta}(g)=\int g h d \mu$ para todo $g \in L_{1}(\mu)$ e para algum $h \in L_{\infty}(\mu)$. Se $x_{0}=e^{h}$, então $\int g \log \left|x_{0}\right| d \mu \leq \Phi_{0}(g)$ para todo $g \in P$, isto é, $x_{0} \in B_{0}$, e $\alpha<\Phi_{0}(f) \leq \bar{\beta}(f)=$ $\int f \log \left|x_{0}\right| d \mu$. Logo,

$$
\Phi_{0}(f)=\sup _{x \in B_{0}} \int f \log |x| d \mu
$$

Definamos agora $\Phi_{1}(f)=\inf \sum_{k=1}^{n}\left(\Lambda\left(f_{k}\right)-\Phi\left(f_{k}\right)\right)$, onde o ínfimo é tomado sobre os $f_{k} \geq 0$, com $f=\sum_{k=1}^{n} f_{k}$. Por um argumento similar ao anterior provamos que

$$
\Lambda(f)-\Phi(f)-M\|f\|_{L_{1}} \leq \Phi_{1}(f) \leq \Lambda(f)-\Phi(f),
$$

e se $B_{1}$ é o conjunto de todas as funções $x \in L_{0}$, tais que

$$
\int f \log |x| d \mu \leq \Phi_{1}(f) \forall f \in P,
$$

demonstramos novamente que

$$
\Phi_{1}(f)=\sup _{x \in B_{1}} \int f \log |x| d \mu
$$

Provaremos que $\Phi_{1}+\Phi_{2} \leq \Lambda$. De fato, sejam $x \in B_{0}$ e $y \in B_{1}$. Então para $f \in P$, obtemos que

$$
\begin{aligned}
\int f \log |x| d \mu+\int f \log |y| d \mu & =\int f \log |x \cdot y| d \mu \\
& \leq \Lambda(f)
\end{aligned}
$$




\subsection{Aproximação de funcionais semilineares}

logo, pelo Teorema 6.17, obtemos que $x \cdot y \in B_{L_{1}}$, concluindo que $B_{0} \cdot B_{1} \subseteq B_{L_{1}}$. Seja $B_{X}=\overline{C\left(B_{1}\right)}$ a envoltória convexa fechada na topologia q.s. de $B_{1}$. Argumentando como na prova do Teorema 6.20, demonstra-se que $B_{X} \cdot B_{1} \subseteq B_{L_{1}}$. Definimos um espaço $X$, com uma função norma

$$
\|x\|_{X}= \begin{cases}\inf \left\{\alpha>0: \alpha^{-1} x \in B_{X}\right\}, & \text { se } \alpha^{-1} x \in B_{X}, \text { para algum } \alpha>0 \\ \infty, & \text { se } \alpha^{-1} x \notin B_{X}, \text { para todo } \alpha>0 .\end{cases}
$$

No Teorema 6.20 demonstramos que o $X$ assim definido é um espaço de Köthe. Usando o fato $B_{X} \cdot B_{1} \subseteq B_{L_{1}}$, provamos que

$$
\Phi_{X}(f)+\Phi_{1}(f) \leq \Lambda(f), \quad \forall f \in P
$$

Agora das desigualdades (6.23) e (6.24), segue-se que

$$
\Phi_{X}(f) \leq \Phi(f)+M\|f\|_{L_{1}}, \quad \forall f \in P .
$$

Como $B_{0} \subseteq B_{X}$, então $\Phi_{0}(f) \leq \Phi_{X}(f)$ para todo $f \in P$ e pela desigualdade (6.18), demonstramos $\Phi(f)-M\|f\|_{L_{1}} \leq \Phi_{X}(f)$ para todo $f \in P$. Até o momento Provamos que existe um espaço de Köthe $X$, tal que

$$
\left|\Phi_{X}(f)-\Phi(f)\right| \leq M\|f\|_{L_{1}}, \quad \forall f \in P .
$$

Se $f \geq 0$, existe uma sequência decrescente em $P$, que denotamos por $\left(f_{n}\right)_{n}$, tal que $f_{n} \rightarrow f$ na topologia pontual. O teorema da convergência monótona implica que $\left\|f_{n}\right\|_{L_{1}} \rightarrow 0$, se $n \rightarrow \infty$. Como $\Phi$ é semilinear, o Teorema 6.16 implica que

$$
\limsup _{n \rightarrow \infty}\left|\Phi\left(f_{n}\right)-\Phi(f)\right| \leq 2 \delta(\Phi)\|f\|_{L_{1}}
$$

portanto

$$
\begin{aligned}
\limsup _{n \rightarrow \infty}\left|\Phi_{X}\left(f_{n}\right)-\Phi(f)\right| & \leq \limsup _{n \rightarrow \infty}\left|\Phi\left(f_{n}\right)-\Phi(f)\right|+\limsup _{n \rightarrow \infty}\left|\Phi_{X}\left(f_{n}\right)-\Phi\left(f_{n}\right)\right| \\
& \leq 2 \delta(\Phi)\|f\|_{L_{1}}+M\|f\|_{L_{1}}
\end{aligned}
$$

Consequentemente,

$$
\underset{n \rightarrow \infty}{\limsup }\left|\Phi_{X}\left(f_{n}\right)-\Phi(f)\right| \leq(M+2 \delta(\Phi))\|f\|_{L_{1}} \quad \forall f \geq 0 .
$$

Note que

$$
\left|\Phi_{X}(f)-\Phi(f)\right| \leq\left|\Phi_{X}(f)-\Phi_{X}\left(f_{n}\right)\right|+\left|\Phi_{X}\left(f_{n}\right)-\Phi(f)\right|,
$$

$\log 0$

$$
\left|\Phi_{X}(f)-\Phi(f)\right| \leq(M+2 \delta(\Phi))\|f\|_{L_{1}} \quad \forall f \geq 0 .
$$




\subsection{Aproximação de funcionais semilineares}

Resumindo, existe um espaço de Köthe $X$, tal que

$$
\left|\Phi_{X}(f)-\Phi(f)\right| \leq(M+2 \delta(\Phi))\|f\|_{L_{1}},
$$

para todo $f \in L_{1}^{+}(S)$, onde $S$ é finito.

Caso 2. Suponha que $\mathscr{T}$ seja um semi-ideal estrito de $L_{1}^{+}(S)$, onde $S$ não é necessariamente finito. Seja $\mathscr{A}=\left(A_{1}, \ldots, A_{n}\right)$ uma coleção finita de conjuntos mensuráveis disjuntos tais que $1_{A_{k}} \in \mathscr{T}$, para cada $1 \leq k \leq n$. Consideremos $E_{\mathscr{A}}=\left\{\sum_{K=1}^{n} c_{k} \cdot 1_{A_{k}}: c_{k} \geq 0\right\}$. Demonstraremos que existe um funcional semilinear convexo $\Psi_{\mathscr{A}}$, definido em $E_{\mathscr{A}}$, tal que $\Lambda-\Psi_{\mathscr{A}}$ é convexo e

$$
\left|\Psi_{\mathscr{A}}(f)-\Phi(f)\right| \leq(M+2 \delta(\Phi))\|f\|_{L_{1}},
$$

para todo $f \in E_{\mathscr{A}}$. De fato, seja $S_{n}=\left\{s_{1}, \ldots, s_{n}\right\}$ e $\left(S_{n}, \mathscr{P}\left(S_{n}\right), v\right)$ um espaço de medida onde $v\left(\left\{s_{k}\right\}\right)=\mu\left(A_{k}\right)$, para cada $1 \leq k \leq n$. Definamos uma função $\pi: E_{\mathscr{A}} \longrightarrow L_{1}^{+}\left(S_{n}, v\right)$ pondo

$$
\sum_{k=1}^{n} c_{k} \cdot 1_{A_{k}} \longmapsto \sum_{k=1}^{n} c_{k} \cdot 1_{\left\{s_{k}\right\}} .
$$

É imediato verificar que $\pi$ satisfaz as seguintes propriedades:

- Se $f, g \in E_{\mathscr{A}}$ e $\lambda \geq 0$, então $\pi(f+\lambda \cdot g)=\pi(f)+\lambda \cdot \pi(g)$.

- A função $\pi$ é bijetora.

- $\|f\|_{L_{1}(S, \mu)}=\|\pi(f)\|_{L_{1}\left(S_{n}, v\right)}$.

- Se denotamos como $\Lambda_{L_{1}\left(S_{n}, v\right)}=\Phi_{L_{1}\left(S_{n}, v\right)}$, então

$$
\Lambda\left(\pi^{-1}(f)\right)=\Lambda_{L_{1}\left(S_{n}, v\right)}(f) \text {, para todo } f \in L_{1}^{+}\left(S_{n}, v\right) .
$$

Seja $\Phi_{E_{\mathscr{A}}}=\Phi \uparrow_{E_{\mathscr{A}}}$. Então $\Phi_{E_{\mathscr{A}}} \circ \pi^{-1}: L_{1}^{+}\left(S_{n}, v\right) \longrightarrow \mathbb{C}$ é um funcional semilinear sobre $L_{1}^{+}\left(S_{n}, v\right)$. Mais ainda

$$
\begin{aligned}
\Delta_{\Phi_{E_{\mathscr{A}}} \circ \pi^{-1}}\left(f_{1}, \ldots, f_{n}\right) & =\sum_{k=1}^{n}\left(\Phi_{E_{\mathscr{A}}} \circ \pi^{-1}\right)\left(f_{k}\right)-\left(\Phi_{E_{\mathscr{A}}} \circ \pi^{-1}\right)\left(\sum_{k=1}^{n} f_{k}\right) \\
& =\sum_{k=1}^{n} \Phi_{E_{\mathscr{A}}}\left(\pi^{-1}\left(f_{k}\right)\right)-\Phi_{E_{\mathscr{A}}}\left(\sum_{k=1}^{n} \pi^{-1}\left(f_{k}\right)\right)
\end{aligned}
$$




$$
\begin{aligned}
& =\Delta_{\Phi_{E_{\mathscr{A}}}}\left(\pi^{-1}\left(f_{1}\right), \ldots, \pi^{-1}\left(f_{n}\right)\right) \\
& \leq M \sum_{k=1}^{n}\left\|\pi^{-1}\left(f_{k}\right)\right\|_{L_{1}(S)}+\Delta_{\Lambda}\left(\pi^{-1}\left(f_{1}\right), \ldots, \pi^{-1}\left(f_{n}\right)\right) \\
& =M \sum_{k=1}^{n}\left\|f_{k}\right\|_{L_{1}^{+}\left(S_{n}, v\right)}+\Delta_{\Lambda_{L_{1}\left(S_{n}, v\right)}}\left(f_{1}, \ldots, f_{n}\right)
\end{aligned}
$$

para todo $f_{1}, \ldots, f_{n} \in L_{1}\left(S_{n}, v\right)$. Similarmente se prova que

$$
\Delta_{\Phi_{E_{\mathscr{A}}} \circ \pi^{-1}}\left(f_{1}, \ldots, f_{n}\right) \geq-M \sum_{k=1}^{n}\left\|f_{k}\right\|_{L_{1}^{+}\left(S_{n}, v\right)} .
$$

para todo $f_{1}, \ldots, f_{n} \in L_{1}\left(S_{n}, v\right)$.

Até o momento, demonstramos que

$$
-M \sum_{k=1}^{n}\left\|f_{k}\right\|_{L_{1}^{+}\left(S_{n}, v\right)} \leq \Delta_{\Phi_{E_{\mathscr{A}}} \circ \pi^{-1}}\left(f_{1}, \ldots, f_{n}\right) \leq M \sum_{k=1}^{n}\left\|f_{k}\right\|_{L_{1}^{+}\left(S_{n}, v\right)}+\Delta_{\Lambda_{L_{1}\left(S_{n}, v\right)}}\left(f_{1}, \ldots, f_{n}\right),
$$

para todo $f_{1}, \ldots, f_{n} \in L_{1}\left(S_{n}, v\right)$. Portanto o funcional semilinear $\Phi_{E_{\mathscr{A}}} \circ \pi^{-1}$ verifica as condições do Caso 1., assim existe um espaço de Köthe $X$, tal que

$$
\left|\Phi_{X}(g)-\left(\Phi_{E_{\mathscr{A}}} \circ \pi^{-1}\right)(g)\right| \leq(M+2 \delta(\Phi))\|g\|_{L_{1}},
$$

para todo $g \in L_{1}^{+}\left(S_{n}, v\right)$.

Seja $g=\pi(f)$, onde $f \in E_{\mathscr{A}}$, definimos $\Psi_{\mathscr{A}}$ por $\Psi_{\mathscr{A}}(f)=\Phi_{X}(\pi(f))$. O funcional $\Psi_{\mathscr{A}}$ assim definido é um funcional semilinear convexo com $\Lambda-\Psi_{\mathscr{A}}$ convexo (lembre-se que $\Phi_{X}$ e $\Lambda_{L_{1}^{+}\left(S_{n}, v\right)}$ $\Phi_{X}$ são convexos e $\pi$ linear, portanto $\Psi_{\mathscr{A}}$ e $\Lambda-\Psi_{\mathscr{A}}$ são convexos) tal que

$$
\left|\Psi_{\mathscr{A}}(f)-\Phi(f)\right| \leq(M+2 \delta(\Phi))\|f\|_{L_{1}},
$$

para todo $f \in E_{\mathscr{A}}$.

Caso 3. Seja $\mathscr{U}$ o cone das funções simples em $\mathscr{T}$. Então existe um funcional semilinear convexo $\Psi_{\mathscr{U}}$, definido em $\mathscr{U}, \operatorname{com} \Lambda-\Psi_{\mathscr{U}}$ convexo tal que

$$
\left|\Psi_{\mathscr{U}}(f)-\Phi(f)\right| \leq(M+2 \delta(\Phi))\|f\|_{L_{1}},
$$

para todo $f \in \mathscr{U}$. De fato, seja $f \in \mathscr{U}$. Note que $f \in E_{\mathscr{A}}$ para algum espaço $E_{\mathscr{A}}$. Representemos a partição $\left\{A_{1}, \ldots, A_{n},\left(S \backslash \cup_{k=1}^{n} A_{k}\right)\right\}$ de $S$ por $\mathscr{A}=\left(A_{1}, \ldots, A_{n}\right)$. Seja $\mathscr{P}$ o conjunto de todas 


\subsection{Aproximação de funcionais semilineares}

as partições de $S$. O espaço $E_{\mathscr{A}}$, onde $\mathscr{A}=\left(A_{1}, \ldots, A_{n}\right)$ e os $A_{k}$ são conjuntos mensuráveis disjuntos, será como no caso anterior, o conjunto das funções $f \in \mathscr{U}$ tais que $f=\sum_{k=1}^{n} c_{k} \cdot 1_{A_{k}}$, para constantes $c_{k} \geq 0$. Definamos uma relação $\geq$ em $\mathscr{P}$ como: se $\mathscr{A}=\left(A_{1}, \ldots, A_{n}\right), \mathscr{A}^{\prime}=$ $\left(A_{1}^{\prime}, \ldots, A_{m}^{\prime}\right) \in \mathscr{P}$, então $\mathscr{A}^{\prime} \geq \mathscr{A}$ se, e somente se, $\mathscr{A}^{\prime}$ é mais fina que $\mathscr{A}$. O par $(\mathscr{P}, \geq)$ é um conjunto direito. Portanto, para $f \in \mathscr{U}$ definimos uma rede $\Upsilon_{f}: \mathscr{P} \longrightarrow \mathbb{R}$ por

$$
\Upsilon_{f}(\mathscr{A})= \begin{cases}\Phi(f), & \text { se } f \notin E_{\mathscr{A}} \\ \Psi_{\mathscr{A}}(f), & \text { se } f \in E_{\mathscr{A}} .\end{cases}
$$

Por Abuso de notação, representamos a rede $\Upsilon_{f}$ por $\left(\Psi_{\mathscr{A}}(f)\right)_{\mathscr{A} \in \mathscr{P}}$. Então pelo caso Caso 2. e pela forma como definimos $\left(\Psi_{\mathscr{A}}(f)\right)_{\mathscr{A} \in \mathscr{P}}$ temos que

$$
\left|\Psi_{\mathscr{A}}(f)-\Phi(f)\right| \leq(M+2 \delta(\Phi))\|f\|_{L_{1}},
$$

para todo $\mathscr{A} \in \mathscr{P}$, onde $f \in \mathscr{U}$ é fixo. A desigualdade acima mostra que ( $\left.\Psi_{\mathscr{A}}(f)\right)_{\mathscr{A} \in \mathscr{P}}$ é uma rede limitada, portanto, existe uma subrede convergente de $\left(\Psi_{\mathscr{A}}(f)\right)_{\mathscr{A} \in \mathscr{P}}, \log$ o o conjunto

$$
D_{f}=\left\{\left(\Psi_{\mathscr{A}^{\prime}}(f)\right)_{\mathscr{A}^{\prime} \in \mathscr{P}} \text { subrede de }\left(\Psi_{\mathscr{A}}(f)\right)_{\mathscr{A} \in \mathscr{P}}:\left(\Psi_{\mathscr{A}^{\prime}}(f)\right)_{\mathscr{A}^{\prime} \in \mathscr{P}} \text { é convergente }\right\}
$$

é não vazio. Consideremos a família indexada $\left(D_{f}\right)_{f \in \mathscr{U}}$. Pelo axioma da escolha, existe uma funcão $e: \mathscr{U} \longrightarrow \cup_{f \in \mathscr{U}} D_{f}$ tal que $e(f) \in D_{f}$. Denotemos $e(f)=\left(\Psi_{\mathscr{A}^{\prime}}(f)\right)_{\mathscr{A}^{\prime} \in \mathscr{P}}$. Agora definamos um funcional semilinear $\Psi_{\mathscr{U}}: \mathscr{U} \longrightarrow \mathbb{R}$ pondo

$$
\Psi_{\mathscr{U}}(f)=\lim _{\mathscr{A}^{\prime}} \Psi_{\mathscr{A}^{\prime}}(f) .
$$

Observe que $\Psi_{\mathscr{U}}$ assim definido verifica que

$$
\left|\Psi_{\mathscr{U}}(f)-\Phi(f)\right| \leq(M+2 \delta(\Phi))\|f\|_{L_{1}},
$$

para todo $f \in \mathscr{U}$. Sejam $f, g \in \mathscr{U}$. Então existem $\mathscr{A}^{\prime}, \mathscr{A}^{\prime \prime} \in \mathscr{P}$ tais que $f \in E_{\mathscr{A}^{\prime}}$ e $g \in E_{\mathscr{A}^{\prime \prime}}$. Se $\mathscr{A}^{\prime \prime \prime}$ é uma coleção mais fina que $\mathscr{A}^{\prime}$ e $\mathscr{A}^{\prime \prime}$, então $f, g, f+g \in E_{\mathscr{A}}$ '”. Como $\Psi_{\mathscr{A}}$ é convexo para todo $\mathscr{A} \geq \mathscr{A}^{\prime \prime \prime}$, então $\Psi_{\mathscr{A}}(f+g) \leq \Psi_{\mathscr{A}}(f)+\Psi_{\mathscr{A}}(g)$ para todo $\mathscr{A} \geq \mathscr{A}^{\prime \prime \prime}$. Portanto $\Psi_{\mathscr{U}}$ é uma função convexa. Similarmente se demonstra que $\Lambda-\Psi_{\mathscr{U}}$ é uma função convexa. Consequentemente existe um funcional semilinear convexo $\Psi_{\mathscr{U}}$, definido em $\mathscr{U}$, com $\Lambda-\Psi_{\mathscr{U}}$ convexo tal que

$$
\left|\Psi_{\mathscr{U}}(f)-\Phi(f)\right| \leq(M+2 \delta(\Phi))\|f\|_{L_{1}},
$$

para todo $f \in \mathscr{U}$.

Caso 4. Demonstraremos que existe um funcional semilinear convexo $\Psi$, com $\Lambda-\Psi$ convexo, definido em $\mathscr{T}$ tal que

$$
|\Psi(f)-\Phi(f)| \leq(M+2 \delta(\Phi))\|f\|_{L_{1}}
$$




\subsection{Aproximação de funcionais semilineares}

para todo $f \in \mathscr{T}$. De fato, seja $f \in \mathscr{T}$. Como o conjunto das funções simples é denso em $L_{1}(\mu)$, então $\mathscr{U} \cap[0, f]$ é denso em $[0, f]$. Vejamos que o funcional $\Psi_{\mathscr{U}}$ é uniformemente contínuo em $\mathscr{U} \cap[0, f]$. Como $\Phi$ é semilinear, então $\Phi$ é contínua em 0 , quando $\Phi$ é restrito a $[0, f]$ (ver condição 3. da definição de funcional semilinear). Observe que

$$
\left|\Psi_{\mathscr{U}}(g)\right| \leq(M+2 \delta(\Phi))\|g\|_{L_{1}}+|\Phi(g)|
$$

para todo $g \in \mathscr{U} \cap[0, f]$.

Sejam $g, h \in[0, f]$ e $k=\max (g, h)$. Então pelo fato de $\Lambda-\Psi_{\mathscr{U}}$ ser convexo, temos que

$$
\begin{aligned}
\left|\Psi_{\mathscr{U}}(g)-\Psi_{\mathscr{U}}(h)\right|= & \left|\Psi_{\mathscr{U}}(g)+\Psi_{\mathscr{U}}(k-g)-\Psi_{\mathscr{U}}(k)\right| \\
& +\left|\Psi_{\mathscr{U}}(h)+\Psi_{\mathscr{U}}(k-h)-\Psi_{\mathscr{U}}(k)\right|+\left|\Psi_{\mathscr{U}}(k-h)\right|+\left|\Psi_{\mathscr{U}}(k-g)\right| \\
= & \left|\Delta_{\Psi_{\mathscr{U}}}(g, k-g)\right|+\left|\Delta_{\Psi_{\mathscr{U}}}(h, k-h)\right|+\left|\Psi_{\mathscr{U}}(k-h)\right|+\left|\Psi_{\mathscr{U}}(k-g)\right|, \\
\leq & \left|\Delta_{\Lambda}(g, k-g)\right|+\left|\Delta_{\Lambda}(h, k-h)\right|+\left|\Psi_{\mathscr{U}}(k-h)\right|+\left|\Psi_{\mathscr{U}}(k-g)\right| \\
\leq & \left|\Delta_{\Lambda}(g, k-g)\right|+\left|\Delta_{\Lambda}(h, k-h)\right|+(M+2 \delta(\Phi))|| k-\left.h\right|_{L_{1}}+|\Phi(k-h)| \\
& +(M+2 \delta(\Phi))|| k-g \|_{L_{1}}+|\Phi(k-g)| \\
= & \left|\Delta_{\Lambda}(g, k-g)\right|+\left|\Delta_{\Lambda}(h, k-h)\right|+|\Phi(k-h)|+|\Phi(k-g)| \\
& +(M+2 \delta(\Phi))|| h-g \|_{L_{1}}
\end{aligned}
$$

Note que

$$
\begin{aligned}
\left|\Delta_{\Lambda}(g, k-g)\right| & =\|g\|_{L_{1}} \cdot\left|\Delta_{\Lambda}\left(\frac{g}{\|g\|_{L_{1}}}, \frac{k-g}{\|g\|_{L_{1}}}\right)\right| \\
& \leq\|f\|_{L_{1}} \cdot \sup _{\|F\|_{L_{1}} \leq 1} \sup _{\|G\|_{L_{1}} \leq\|k-g\|_{L_{1}}}\left|\Delta_{\Lambda}(F, G)\right|,
\end{aligned}
$$

$\log 0$

$$
\left|\Delta_{\Lambda}(g, k-g)\right| \leq\|f\|_{L_{1}} \cdot \phi\left(\|k-g\|_{L_{1}}\right)
$$

onde

$$
\phi(\lambda)=\sup _{\|F\|_{L_{1}} \leq 1} \sup _{\|G\|_{L_{1}} \leq \lambda}\left|\Delta_{\Lambda}(F, G)\right|
$$

e $\lim _{\lambda \rightarrow 0} \phi(\lambda)=0$, pois $\Lambda$ é um funcional semilinear $K$-contínuo. Similarmente 


$$
\left|\Delta_{\Lambda}(h, k-h)\right| \leq\|f\|_{L_{1}} \cdot \phi_{0}\left(\|h-g\|_{L_{1}}\right),
$$

onde $\phi_{0}$ é uma função tal que $\lim _{\lambda \rightarrow 0} \phi_{0}(\lambda)=0$. Isto prova que

$$
\begin{aligned}
\left|\Psi_{\mathscr{U}}(g)-\Psi_{\mathscr{U}}(h)\right| & \leq\|f\|_{L_{1}} \cdot \phi\left(\|k-g\|_{L_{1}}\right)+\|f\|_{L_{1}} \cdot \phi_{0}\left(\|h-g\|_{L_{1}}\right) \\
& +(M+2 \delta(\Phi)) \cdot\|h-g\|_{L_{1}}+|\Phi(k-g)|+|\Phi(k-h)|
\end{aligned}
$$

Agora seja $\varepsilon>0$ arbitrário. Então, existe $\zeta>0$ tal que $0<\lambda<\zeta$ implica que

$$
\begin{gathered}
\phi(\lambda)<\frac{\varepsilon}{5\|f\|_{L_{1}}}, \\
\phi_{0}(\lambda)<\frac{\varepsilon}{5\|f\|_{L_{1}}},
\end{gathered}
$$

e,

$$
|\Phi(l)|<\frac{\varepsilon}{5} \text {, se }\|l\|_{L_{1}}<\zeta \text { e } l \in[0, f] .
$$

Escolha $0<\zeta<\frac{\varepsilon}{5(M+2 \delta(\Phi))}$.

Se $\|h-g\|_{L_{1}}<\zeta$, então como $|k-h|,|k-g| \leq|g-h|$, obtemos imediatamente que $\|k-h\|_{L_{1}}, \| k-$ $g \|_{L_{1}} \leq \zeta$, portanto,

$$
\begin{aligned}
\left|\Psi_{\mathscr{U}}(g)-\Psi_{\mathscr{U}}(h)\right| & \leq \frac{\varepsilon}{5}+\frac{\varepsilon}{5}+\frac{\varepsilon}{5}+\frac{\varepsilon}{5}+\frac{\varepsilon}{5} \\
& =\varepsilon .
\end{aligned}
$$

Resumindo, para cada $\varepsilon>0$ existe $\zeta>0$ tal que

$$
\|g-h\|_{L_{1}}<\zeta \Rightarrow\left|\Psi_{\mathscr{U}}(g)-\Psi_{\mathscr{U}}(h)\right|<\varepsilon .
$$

Isto prova que $\Psi_{\mathscr{U}}$ é uniformemente contínua em $\mathscr{U} \cap[0, f]$. Portanto, o Teorema 2.80 implica que existe uma única extensão uniformemente contínua de $\Psi_{\mathscr{U}}$ a $[0, f]$, que denotamos por $\Psi_{\mathscr{\ell}}$. Definamos $\Psi$ em $\mathscr{T}$ como

$$
\Psi(f)=\lim _{n \rightarrow \infty} \Psi_{\mathscr{U}}\left(f_{n}\right) \text {, onde os } f_{n} \in[0, f] \text { e } f_{n} \stackrel{\|\cdot\| L_{L_{1}}}{\longrightarrow} f .
$$

O Teorema 2.80 garante que a função está bem definida. Note que $\Psi$ e $\Lambda-\Psi$ são funcionais semilineares convexos em $\mathscr{T}$, pois $\Psi_{\mathscr{U}}$ e $\Lambda-\Psi_{\mathscr{U}}$ são funcionais semilineares convexos em $\mathscr{U}$. 
Seja $\left(f_{n}\right)_{n}$ uma sequência de funções simples de $\mathscr{T}$, tal que $f_{n} \rightarrow f$ em norma $\|\cdot\|_{L_{1}}$, com $f_{n} \leq f$. Portanto

$$
\begin{aligned}
\left|\Psi_{\mathscr{U}}\left(f_{n}\right)-\Phi(f)\right| & \leq\left|\Psi_{\mathscr{U}}\left(f_{n}\right)-\Phi\left(f_{n}\right)\right|+\left|\Phi\left(f_{n}\right)-\Phi(f)\right| \\
& \leq(M+2 \delta(\Phi))\left\|f_{n}\right\|_{L_{1}}+2 \delta(\Phi)\|f\|_{L_{1}} .
\end{aligned}
$$

Se $n \rightarrow \infty$, obtemos que

$$
|\Psi(f)-\Phi(f)| \leq(M+4 \delta(\Phi))\|f\|_{L_{1}}
$$

para todo $f \in \mathscr{T}, \operatorname{logo} d(\Phi, \Psi) \leq M+4 \delta(\Phi)$. O Teorema 6.20 implica que existe um espaço de Köthe tal que $\Psi=\Phi_{X}$. $\operatorname{Logo} d\left(\Phi, \Phi_{X}\right) \leq M+4 \delta(\Phi)$.

Teorema 6.22 Sejam $S$ um conjunto e $\mu$ uma medida positiva definida em uma $\sigma$-álgebra finita de $S$, que denotamos por $\Sigma$. Se $\phi: \Sigma \longrightarrow \mathbb{R}$ é uma função que satisfaz

$$
\phi(A)+\phi(B)-\mu(A \cup B) \leq \phi(A \cup B) \leq \phi(A)+\phi(B)
$$

para todo $A, B \in \Sigma$ disjuntos, então existe uma medida com sinal $v$ em $\Sigma$ tal que $v(A) \leq \phi(A)$ para todo $A \in \Sigma$ e $v(S) \geq \phi(S)-4 \mu(S)$.

Demonstração. Para $A \in \Sigma$, definamos um $\sigma$-álgebra em $A$ como $\Sigma_{A}=\{D \in \Sigma: D \subseteq A\}$. Seja $\Gamma_{A}$ o conjunto de todas as medidas com sinal $\rho$, definidas em $\left(A, \Sigma_{A}\right)$, tais que $\rho \leq \phi$. Seja $h: \Sigma \longrightarrow \mathbb{R}$ definida por

$$
h(A)= \begin{cases}\mu(A)^{-1}\left(\phi(A)-\sup _{\rho \in \Gamma_{A}} \rho(A)\right), & \text { se } \mu(A) \neq 0 \\ 0, & \text { se } \mu(A)=0 .\end{cases}
$$

Então, para cada $n \in \mathbb{N}$, existe $v_{n} \in \Gamma_{S}$, tal que $\sup _{\rho \in \Gamma_{S}} \rho(S)-1 / n \leq v_{n}(S)$, que equivale a

$$
\phi(S)-h(S) \mu(S)-1 / n \leq v_{n}(S), \forall n \in \mathbb{N} .
$$

Observe que a sequência $\left(v_{n}(A)\right)_{n}$ é limitada para todo $A \in \Sigma$, pois $v_{n}(A) \leq \phi(A)$ para todo $n \in \mathbb{N}$ e

$$
\begin{aligned}
v_{n}(A) & =v_{n}(S)-v_{n}(S \backslash A) \\
& \geq \phi(S)-h(S) \mu(S)-1 / n-\phi(S \backslash A) \\
& \geq \phi(S)-h(S) \mu(S)-1-\phi(S \backslash A)
\end{aligned}
$$




\subsection{Aproximação de funcionais semilineares}

para todo $n \in \mathbb{N}$. Como $\Sigma$ é finito, então existe uma subsequência de $\left(v_{n}\right)_{n}$, que denotamos por $\left(v_{n}\right)_{n}$, tal que para todo $A \in \Sigma$ a sequência $\left(v_{n}(A)\right)_{n}$ converge. Se definimos uma medida com sinal como $v(A)=\lim _{n \rightarrow \infty} v(A)$, temos que $v(S) \geq \phi(S)-h(S) \mu(S)$ e $v(A) \leq \phi(A)$ para todo $A \in \Sigma$. Para terminar a prova, basta mostrar que $\gamma=\max _{A \in \Sigma} h(A) \leq 4$, pois $\gamma(S) \geq \phi(S)-\gamma \mu(S) \geq$ $\phi(S)-4 \mu(S)$. De fato, assuma que $\max _{A \in \Sigma} h(A)=h(C)$, para algum $C \in \Sigma$.

Se $\mu(C)=0$, então $h(C)=0$, logo, trivialmente $\gamma \leq 4$.

Suponha $\mu(C)>0$. Então, pela definição de $h$, obtemos que para $0<\varepsilon<\mu(C)$, existe $\lambda \in \Gamma_{C}$ tal que

$$
\lambda(C) \geq \phi(C)-\varepsilon-\gamma \mu(C)
$$

Verifiquemos que a afirmação :

$$
B \in \Sigma_{C}, B \neq \emptyset \Rightarrow \lambda(B)<\phi(B)-2 \mu(B)
$$

é falsa. Assuma que seja verdadeira. Isto implica que $\lambda+2 \mu \in \Gamma_{C}$, e por (6.25) obtemos que

$$
\begin{aligned}
\gamma=h(C) & \leq \mu(C)^{-1}(\phi(C)-\lambda(C)-2 \mu(C)) \\
& \leq \mu(C)^{-1}(\varepsilon+\gamma \mu(C)-2 \mu(C)) .
\end{aligned}
$$

Isto é, $\gamma \leq \mu(C)^{-1}(\varepsilon+\gamma \mu(C)-2 \mu(C))$, que equivale a dizer que $2 \mu(C) \leq \varepsilon$ e que contradiz a escolha de $\varepsilon$. Portanto, existe $B \in \Sigma_{C} \operatorname{com} B \neq \emptyset$, tal que $\lambda(B) \geq \phi(B)-2 \mu(B)$. Isto é, o conjunto

$$
\Theta=\left\{D \in \Sigma_{C}: \lambda(D) \geq \phi(D)-2 \mu(D)\right\}
$$

ordenado por inclusão é não vazio e finito, portanto, possui um elemento maximal não vazio, que denotamos por $E$. Observe que

$$
\lambda(E) \geq \phi(E)-2 \mu(E) .
$$

Se $C \backslash E=\emptyset$, então $C=E$, logo por (6.26), temos a desigualdade $4 \geq 2 \geq \mu(C)^{-1}(\phi(C)-\lambda(C)) \geq$ $h(C)$, e novamente estaría provado o teorema.

Suponha que $C \backslash E \neq \emptyset$. Então para todo $A \in \Sigma_{C \backslash E}$, obtemos que $\lambda(E \cup A)<\phi(E \cup A)-2 \mu(E \cup A)$ (lembre-se que $E$ é um elemento maximal de $\Theta$ ). Como $E \cap A=\emptyset$, temos que $\lambda(A \cup E)=$ $\lambda(A)+\lambda(E)$. Usando (6.26) e a hipóteses do teorema, temos que 


$$
\begin{aligned}
\lambda(A) & <\phi(A \cup E)-2 \mu(A \cup E)-\lambda(E) \\
& =\phi(A \cup E)-\phi(E)-2 \mu(A)-(\lambda(E)+2 \mu(E)-\phi(E)) \\
& \leq \phi(A \cup E)-\phi(E)-2 \mu(A) \\
& \leq \phi(A)-2 \mu(A)
\end{aligned}
$$

Resumindo,

$$
\lambda(A)<\phi(A)-2 \mu(A), \text { para todo } A \in \Sigma_{C \backslash E} .
$$

Agora definamos uma medida com sinal em $\left(C, \Sigma_{C}\right)$, que denotamos por $\lambda_{0}$, como

$$
\lambda_{0}(B)=\lambda(B)+\mu(B \cap C \backslash E)-\mu(B \cap E) .
$$

Então, pela desigualdade $\lambda \leq \phi$, por (6.27) e pela hipóteses do teorema, temos que

$$
\begin{aligned}
\lambda_{0}(B) & =\lambda(B)+\mu(B \cap C \backslash E)-\mu(B \cap E) \\
& =\lambda((B \cap E) \cup(B \cap C \backslash E))+\mu(B \cap C \backslash E)-\mu(B \cap E) \\
& =\lambda(B \cap E)+\lambda(B \cap C \backslash E)+\mu(B \cap C \backslash E)-\mu(B \cap E) \\
& \leq \phi(B \cap E)+\lambda(B \cap C \backslash E)+\mu(B \cap C \backslash E)-\mu(B \cap E) \\
& <\phi(B \cap E)+(\phi(B \cap C \backslash E)-2 \mu(B \cap C \backslash E))+\mu(B \cap C \backslash E)-\mu(B \cap E) \\
& =\phi(B \cap E)+\phi(B \cap C \backslash E)-\mu(B \cap C \backslash E)-\mu(B \cap E) \\
& =\phi(B \cap E)+\phi(B \cap C \backslash E)-\mu(B) \\
& \leq \phi(B),
\end{aligned}
$$

isto mostra que $\lambda_{0} \in \Gamma_{C}$. Portanto,

$$
\gamma=h(C) \leq \mu(C)^{-1}\left(\phi(C)-\lambda_{0}(C)\right),
$$




\subsection{Aproximação de funcionais semilineares}

assim $\lambda_{0}(C) \leq \phi(C)-\gamma \mu(C)$ e pela definição de $\lambda_{0}$, temos que $\lambda_{0}(C)=\lambda(C)+\mu(C \backslash E)-\mu(E)$, portanto, $\lambda(C)+\mu(C \backslash E)-\mu(E) \leq \phi(C)-\gamma \mu(C)$, usando a equação (6.25), segue-se que $\phi(C)-$ $\varepsilon-\gamma \mu(C)+\mu(C \backslash E)-\mu(E) \leq \phi(C)-\gamma \mu(C)$, que equivale a $\mu(C \backslash E)-\mu(E) \leq \varepsilon$, deduzindo que

$$
\frac{\mu(C)-\varepsilon}{2} \leq \mu(E)
$$

Pela definição de $h$, existe uma medida com sinal $\vartheta \in \Gamma_{C \backslash E}$, tal que

$$
\vartheta(C \backslash E) \geq \phi(C \backslash E)-\gamma \mu(C \backslash E)-\varepsilon .
$$

Definamos uma medida com sinal em $\left(C, \Sigma_{C}\right)$ por

$$
\vartheta_{0}(B)=\lambda(B \cap E)+\vartheta(B \cap C \backslash E)-\mu(E) .
$$

Usando as desigualdades $\lambda \leq \phi, \vartheta \leq \phi$ e as hipóteses do teorema, temos que para todo $B \in \Sigma_{C}$,

$$
\begin{aligned}
\vartheta_{0}(B) & =\lambda(B \cap E)+\vartheta(B \cap C \backslash E)-\mu(E) \\
& \leq \phi(B \cap E)+\phi(B \cap C \backslash E)-\mu(B) \\
& \leq \phi(B),
\end{aligned}
$$

portanto, $\vartheta_{0} \in \Gamma_{C}$. Note que $\vartheta_{0}(C)=\lambda(E)+\vartheta(C \backslash E)-\mu(C)$ (ver definição de $\vartheta_{0}$ ). Agora

$$
\begin{aligned}
\gamma & =h(C) \\
& \leq \mu(C)^{-1}\left(\phi(C)-\vartheta_{0}(C)\right) \\
& =\mu(C)^{-1}(\phi(C)-\lambda(E)-\vartheta(C \backslash E)+\mu(C)) .
\end{aligned}
$$

Logo,

$$
\lambda(E)+\vartheta(C \backslash E)-\mu(C) \leq \phi(C)-\gamma \mu(C) .
$$

Observe que de (6.29) e (6.26), deduzimos que

$$
\begin{aligned}
\phi(C)-\gamma \mu(C) & \geq \lambda(E)+\vartheta(C \backslash E)-\mu(C) \\
& \geq \lambda(E)+(\phi(C \backslash E)-\gamma \mu(C \backslash E)-\varepsilon)-\mu(C) \\
& \geq \phi(E)-2 \mu(E)+\phi(C \backslash E)-\gamma \mu(C \backslash E)-\varepsilon-\mu(C),
\end{aligned}
$$




\subsection{Aproximação de funcionais semilineares}

pela hipóteses do teorema, temos que $\phi(C) \leq \phi(E)+\phi(C \backslash E)$, logo

$$
\phi(E)+\phi(C \backslash E)-\gamma \mu(C) \geq \phi(E)-2 \mu(E)+\phi(C \backslash E)-\gamma \mu(C \backslash E)-\varepsilon-\mu(C),
$$

que equivale a

$$
(\gamma-2) \mu(E) \leq \mu(C)+\varepsilon,
$$

e usando (6.28), obtemos que

$$
(\gamma-2) \leq \frac{2(\mu(C)+\varepsilon)}{\mu(C)-\varepsilon}
$$

Se $\varepsilon \rightarrow 0$, concluimos que $\gamma \leq 4$.

Lema 6.23 Seja $\sigma>1$. Então existem constantes $C$ e $C^{\prime}$ que dependem de $\sigma$ tais que se $\mathscr{T} \subset(L \log L)_{+}$é um semi-ideal estrito e $\Phi: \mathscr{T} \rightarrow \mathbb{R} e ́$ um funcional semilinear que satisfaz

$$
\Delta_{\Phi}(f, g) \leq \alpha \log 2\left(\|f\|_{L_{1}}+\|g\|_{L_{1}}\right)
$$

para todo $f, g \in \mathscr{T}$ (respectivamente, para todo $f, g \in \mathscr{T}$ disjuntos). Então para $f=\sum_{k=1}^{n} f_{k}$, temos que

$$
\begin{gathered}
\Delta_{\Phi}\left(f_{1}, \ldots, f_{n}\right) \leq \alpha\left(\sigma \sum_{k=1}^{n}\left\|f_{k}\right\|_{L_{1}} \log \frac{\|f\|_{L_{1}}}{\left\|f_{k}\right\|_{L_{1}}}+C\|f\|_{L_{1}}\right) \\
\Delta_{\Phi}\left(f_{1}, \ldots, f_{n}\right) \leq \alpha\left(2 \sigma \sum_{k=1}^{n}(\log k)\left\|f_{k}\right\|_{L_{1}}+C^{\prime}\|f\|_{L_{1}}\right) .
\end{gathered}
$$

para todo $f_{1}, \ldots, f_{n} \in \mathscr{T}$ (respectivamente, para todo $f_{1}, \ldots, f_{n} \in \mathscr{T}$ disjuntos).

Demonstração. Inicialmente, observe que se $f_{1}, \ldots, f_{n} \in \mathscr{T}$, então as $f_{1}, \ldots, f_{n}$ são não negativas, portanto $\|f\|_{L_{1}}=\sum_{k=1}^{n}\left\|f_{k}\right\|_{L_{1}}$.

Considere a função

$$
h(a)=\sigma\left(a \log \frac{1}{a}+(1-a) \log \frac{1}{1-a}\right)-\log 2
$$

definida para $0<a<1$. Note que $h$ é contínua, decrescente no intervalo $\left[\frac{1}{2}, 1\right]$ e $h(1 / 2)=$ $(\sigma-1) \log 2>0$, portanto, existe $0<\theta_{0}<1$ tal que $h$ é positiva em $\left[\frac{1}{2}, \frac{1}{2}\left(1+\theta_{0}\right)\right]$, assim $h$ atinge um mínimo nesse intervalo, isto é, existe $\tau>0$, tal que

$$
\tau \leq \sigma\left(a \log \frac{1}{a}+(1-a) \log \frac{1}{1-a}\right)-\log 2
$$

para todo $\frac{1}{2} \leq a \leq \frac{1}{2}\left(1+\theta_{0}\right)$.

Agora, como $\Phi$ verifica

$$
\Delta_{\Phi}(f, g) \leq \alpha \log 2\left(\|f\|_{L_{1}}+\|g\|_{L_{1}}\right)
$$




\subsection{Aproximação de funcionais semilineares}

para todo $f, g \in \mathscr{T}$, usando indução demonstraremos que

$$
\Delta_{\Phi}\left(f_{1}, \ldots, f_{n}\right) \leq \alpha(n-1) \log 2 \sum_{k=1}^{n}\left\|f_{k}\right\|_{L_{1}}
$$

para todo $f_{1}, \ldots, f_{n} \in \mathscr{T}$.

Assuma que $f_{1}, \ldots, f_{n} \in \mathscr{T}$, com $\sum_{k=1}^{n}\left\|f_{k}\right\|_{L_{1}}=1$. Então a desigualdade acima mostra que

$$
\Delta_{\Phi}\left(f_{1}, \ldots, f_{n}\right) \leq \alpha \sigma \sum_{k=1}^{n}\left\|f_{k}\right\|_{L_{1}} \log \frac{1}{\left\|f_{k}\right\|_{L_{1}}}+\alpha(n-1) \log 2 .
$$

Logo, existe $c_{n}$ tal que

$$
\Delta_{\Phi}\left(f_{1}, \ldots, f_{n}\right) \leq \alpha\left(\sigma \sum_{k=1}^{n}\left\|f_{k}\right\|_{L_{1}} \log \frac{1}{\left\|f_{k}\right\|_{L_{1}}}+c_{n}\right) .
$$

Escolha $c_{n}$ como o menor número que verifica essa desigualdade.

Seja $\theta=\max _{1 \leq k \leq n}\left\|f_{k}\right\|_{L_{1}}$. Vamos dar estimativas de $\Delta_{\Phi}\left(f_{1}, \ldots, f_{n}\right)$ quando $\theta \leq \theta_{0}$ e $\theta \geq \theta_{0}$. De fato: Suponha primeiro que $\theta \leq \theta_{0}$. Escolhamos para $1 \leq k \leq n$, constantes $\xi_{k}= \pm 1$ tais que $0 \leq \sum_{k=1}^{n} \xi_{k} \cdot\left\|f_{k}\right\|_{L_{1}} \leq \theta$. Sejam $A=\left\{k: \xi_{k}=1\right\}$ e $B=\left\{k: \xi_{k}=-1\right\}$. Consideremos $a=\sum_{k \in A}\left\|f_{k}\right\|_{L_{1}}$. Observe que $2 a-1=\sum_{k=1}^{n} \xi_{k} \cdot\left\|f_{k}\right\|_{L_{1}}$, logo $0 \leq 2 a-1 \leq \theta \leq \theta_{0}$. Isto é, $\frac{1}{2} \leq a \leq \frac{1}{2}\left(1+\theta_{0}\right)$.

Sejam $f_{A}=\sum_{k \in A} f_{k}$ e $f_{B}=\sum_{k \in B} f_{k}$. A hipótese do teorema implica que

$$
\Delta_{\Phi}\left(f_{A}, f_{B}\right) \leq \alpha \log 2 .
$$

Seja $\Delta_{\Phi}\left(f_{k}: k \in A\right)=\Delta_{\Phi}(\overbrace{\left\{f_{k}: k \in A\right\}, 0, \ldots, 0}^{n \text {-termos }})$. Agora, pela desigualdade (6.32), temos que

$$
\Delta_{\Phi}\left(\frac{f_{k}}{a}: k \in A\right) \leq \alpha\left(\sigma \sum_{k \in A} \frac{\left\|f_{k}\right\|_{L_{1}}}{a} \log \frac{a}{\left\|f_{k}\right\|_{L_{1}}}+c_{n}\right),
$$

que equivale a

$$
\Delta_{\Phi}\left(f_{k}: k \in A\right) \leq \alpha\left(\sigma \sum_{k \in A}\left\|f_{k}\right\|_{L_{1}} \log \frac{a}{\left\|f_{k}\right\|_{L_{1}}}+a c_{n}\right)
$$

Similarmente obtemos que

$$
\Delta_{\Phi}\left(f_{k}: k \in B\right) \leq \alpha\left(\sigma \sum_{k \in B}\left\|f_{k}\right\|_{L_{1}} \log \frac{1-a}{\left\|f_{k}\right\|_{L_{1}}}+(1-a) c_{n}\right) .
$$

Agora por (6.33), (6.34) e (6.35), temos que 


\subsection{Aproximação de funcionais semilineares}

$$
\begin{aligned}
\Delta_{\Phi}\left(f_{1}, \ldots, f_{n}\right)= & \Delta_{\Phi}\left(f_{A}, f_{B}\right)+\Delta_{\Phi}\left(f_{k}: k \in A\right)+\Delta_{\Phi}\left(f_{k}: k \in B\right) \\
\leq & \alpha \log 2+\alpha\left(\sigma \sum_{k \in A}\left\|f_{k}\right\|_{L_{1}} \log \frac{a}{\left\|f_{k}\right\|_{L_{1}}}+a c_{n}\right) \\
& +\alpha\left(\sigma \sum_{k \in B}\left\|f_{k}\right\|_{L_{1}} \log \frac{1-a}{\left\|f_{k}\right\|_{L_{1}}}+(1-a) c_{n}\right) \\
= & \alpha\left(\log 2+\sigma \sum_{k \in A}\left\|f_{k}\right\|_{L_{1}} \log \frac{a}{\left\|f_{k}\right\|_{L_{1}}}+\sigma \sum_{k \in B}\left\|f_{k}\right\|_{L_{1}} \log \frac{1-a}{\left\|f_{k}\right\|_{L_{1}}}+c_{n}\right)
\end{aligned}
$$

Substituindo $\sigma \sum_{k \in A}\left\|f_{k}\right\|_{L_{1}} \log \frac{a}{\left\|f_{k}\right\|_{L_{1}}}$ por $\sigma \sum_{k=1}^{n}\left\|f_{k}\right\|_{L_{1}} \log \frac{a}{\left\|f_{k}\right\|_{L_{1}}}-\sigma \sum_{k \in B}\left\|f_{k}\right\|_{L_{1}} \log \frac{a}{\left\|f_{k}\right\|_{L_{1}}}$ e pela desigualdade (6.31) temos que

$$
\begin{aligned}
& =\alpha\left(\sigma \sum_{k=1}^{n}\left\|f_{k}\right\|_{L_{1}} \log \frac{1}{\left\|f_{k}\right\|_{L_{1}}}+\sigma(a \log a+(1-a) \log (1-a))+\log 2+c_{n}\right) \\
& \leq \alpha\left(\sigma \sum_{k=1}^{n}\left\|f_{k}\right\|_{L_{1}} \log \frac{1}{\left\|f_{k}\right\|_{L_{1}}}-\tau+c_{n}\right),
\end{aligned}
$$

onde $\tau>0$. Resumindo,

$$
\Delta_{\Phi}\left(f_{1}, \ldots, f_{n}\right) \leq \alpha\left(\sigma \sum_{k=1}^{n}\left\|f_{k}\right\|_{L_{1}} \log \frac{1}{\left\|f_{k}\right\|_{L_{1}}}-\tau+c_{n}\right),
$$

para todo $f_{1}, \ldots, f_{n} \in \mathscr{T}, \operatorname{com} \sum_{k=1}^{n}\left\|f_{k}\right\|_{L_{1}}=1$ e $\theta \leq \theta_{0}$.

Agora suponha que $\theta>\theta_{0}$, e sem perda de generalidade assuma que $\theta=\left\|f_{n}\right\|$. Por cálculos similares dos anteriores deduzimos que

$$
\begin{aligned}
\Delta_{\Phi}\left(f_{1}, \ldots, f_{n}\right) & =\Delta_{\Phi}\left(\sum_{k=1}^{n-1} f_{k}, f_{n}\right)+\Delta_{\Phi}\left(f_{1}, \ldots, f_{n-1}, 0\right) \\
& \leq \alpha \log 2\left(\left\|\sum_{k=1}^{n-1} f_{k}\right\|_{L_{1}}+\left\|f_{k}\right\|_{L_{1}}\right)+\left(\alpha \sigma \sum_{k=1}^{n-1}\left\|f_{k}\right\|_{L_{1}} \log \frac{1-\theta}{\left\|f_{k}\right\|_{L_{1}}}+\alpha c_{n}(1-\theta)\right) \\
& \leq \alpha\left(\sigma \sum_{k=1}^{n}\left\|f_{k}\right\|_{L_{1}} \log \frac{1}{\left\|f_{k}\right\|_{L_{1}}}+c_{n}\left(1-\theta_{0}\right)+\log 2\right) .
\end{aligned}
$$




\subsection{Aproximação de funcionais semilineares}

Resumindo,

$$
\Delta_{\Phi}\left(f_{1}, \ldots, f_{n}\right) \leq \alpha\left(\sigma \sum_{k=1}^{n}\left\|f_{k}\right\|_{L_{1}} \log \frac{1}{\left\|f_{k}\right\|_{L_{1}}}+c_{n}\left(1-\theta_{0}\right)+\log 2\right),
$$

para todo $f_{1}, \ldots, f_{n} \in \mathscr{T}, \operatorname{com} \sum_{k=1}^{n}\left\|f_{k}\right\|_{L_{1}}=1$ e $\theta>\theta_{0}$.

Em Consequentemente, para todo $f_{1}, \ldots, f_{n} \in \mathscr{T}$, com $\sum_{k=1}^{n}\left\|f_{k}\right\|_{L_{1}}=1$, temos que

$$
\Delta_{\Phi}\left(f_{1}, \ldots, f_{n}\right) \leq \alpha\left(\sigma \sum_{k=1}^{n}\left\|f_{k}\right\|_{L_{1}} \log \frac{1}{\left\|f_{k}\right\|_{L_{1}}}+\max \left(c_{n}\left(1-\theta_{0}\right)+\log 2, c_{n}-\tau\right)\right) .
$$

Então pela forma como nós escolhemos $c_{n}$ (ver desigualdade (6.32)), deduzimos que

$$
\max \left(c_{n}\left(1-\theta_{0}\right)+\log 2, c_{n}-\tau\right)=c_{n}\left(1-\theta_{0}\right)+\log 2,
$$

$\operatorname{assim} c_{n} \leq c_{n}\left(1-\theta_{0}\right)+\log 2$, portanto $c_{n} \leq \frac{\log 2}{\theta_{0}}$, o que prova a primeira parte do teorema, isto é,

$$
\Delta_{\Phi}\left(f_{1}, \ldots, f_{n}\right) \leq \alpha\left(\sigma \sum_{k=1}^{n}\left\|f_{k}\right\|_{L_{1}} \log \frac{1}{\left\|f_{k}\right\|_{L_{1}}}+\frac{\log 2}{\theta_{0}}\right),
$$

para todo $f_{1}, \ldots, f_{n} \in \mathscr{T}$, com $\sum_{k=1}^{n}\left\|f_{k}\right\|_{L_{1}}=1$, onde $C=\frac{\log 2}{\theta_{0}}$ depende só de $\sigma$.

A segunda parte do teorema, é um resultado imediato do Lema 2.76. De fato, pela parte anterior, temos que

$$
\Delta_{\Phi}\left(f_{1}, \ldots, f_{n}\right) \leq \alpha\left(\sigma \sum_{k=1}^{n}\left\|f_{k}\right\|_{L_{1}} \log \frac{1}{\left\|f_{k}\right\|_{L_{1}}}+C\right),
$$

para todo $f_{1}, \ldots, f_{n} \in \mathscr{T}$, com $\sum_{k=1}^{n}\left\|f_{k}\right\|_{L_{1}}=1$, e $C$ depende só de $\sigma$. Assumamos sem perda de generalidade que $\left\|f_{1}\right\|_{L_{1}} \geq\left\|f_{2}\right\|_{L_{1}} \geq \ldots \geq\left\|f_{n}\right\|_{L_{1}}$. Se $\xi_{k}=\left\|f_{k}\right\|_{L_{1}}$ e $\varepsilon=1$, no Lema 2.76 temos que

$$
\sum_{k=1}^{n}\left\|f_{k}\right\|_{L_{1}} \log \frac{1}{\left\|f_{k}\right\|_{L_{1}}} \leq 2 \sum_{k=1}^{n}(\log k)\left\|f_{k}\right\|_{L_{1}}+B_{2},
$$

$\log 0$

$$
\Delta_{\Phi}\left(f_{1}, \ldots, f_{n}\right) \leq \alpha\left(2 \sigma \sum_{k=1}^{n}(\log k)\left\|f_{k}\right\|_{L_{1}}+C+B_{2}\right),
$$

para todo $f_{1}, \ldots, f_{n} \in \mathscr{T}$, com $\sum_{k=1}^{n}\left\|f_{k}\right\|_{L_{1}}=1$. Se $C^{\prime}=C+B_{2}$, então $C^{\prime}$ depende só de $\sigma$. Isto mostra a segunda parte do lema. 
Lema 6.24 Dado $\varepsilon>0$, existe uma constante $\beta$ (que depende de $\varepsilon$ ) tal que para todo semiideal $\mathscr{T}$ e $\Phi: \mathscr{T} \longrightarrow \mathbb{R}$ funcional semilinear, temos que

$$
\left|\Delta_{\Phi}\left(f_{1}, \ldots, f_{n}\right)\right| \leq \delta(\Phi)\left(\varepsilon \sum_{k=1}^{n} k\left\|f_{k}\right\|_{L_{1}}+\beta\left\|\sum_{k=1}^{n} f_{k}\right\|_{L_{1}}\right) .
$$

Demonstração. Seja $\varepsilon>0$ dado. Como $\Phi$ é semilinear, temos que

$$
\Delta_{\Phi}(f, g) \leq \frac{\delta(\Phi)}{\log 2} \cdot \log 2\left(\|f\|_{L_{1}}+\|g\|_{L_{1}}\right)
$$

Agora aplicando o Lema 6.23, $\operatorname{com} \sigma=2 \log 2>1$ e $\alpha=\frac{\delta(\Phi)}{\log 2}$, temos que

$$
\Delta_{\Phi}\left(f_{1}, \ldots, f_{n}\right) \leq \frac{\delta(\Phi)}{\log 2}\left(4 \log 2 \sum_{k=1}^{n}(\log k)\left\|f_{k}\right\|_{L_{1}}+C^{\prime}\left\|\sum_{k=1}^{n} f_{k}\right\|_{L_{1}}\right),
$$

para todo $f_{1}, \ldots, f_{n} \in \mathscr{T}$, onde $C^{\prime}$ é constante.

Afirmamos que para $\varepsilon>0$, existe un número real $\gamma(\varepsilon)$ tal que para todo $k \in \mathbb{N}$, temos que

$$
\log k \leq k \cdot \varepsilon+\gamma(\varepsilon)
$$

De fato, como $\lim _{k \rightarrow \infty} \frac{\log k}{k}=0$, então existe $N_{\varepsilon} \in \mathbb{N}$, tal que

$$
\text { se } k \geq N_{\varepsilon} \Rightarrow \log k \leq k \cdot \varepsilon \text {. }
$$

Basta definir $\gamma(\varepsilon)=\sum_{m=1}^{N_{\varepsilon}} \log m$. Agora da desigualdade (6.36), deduzimos que

$$
\begin{aligned}
\Delta_{\Phi}\left(f_{1}, \ldots, f_{n}\right) & \leq \delta(\Phi)\left(4 \sum_{k=1}^{n}(\varepsilon k+\gamma(\varepsilon))\left\|f_{k}\right\|_{L_{1}}+C^{\prime}\left\|\sum_{k=1}^{n} f_{k}\right\|_{L_{1}}\right) \\
& =\delta(\Phi)\left(\sum_{k=1}^{n} 4 \varepsilon k\left\|f_{k}\right\|_{L_{1}}+\left(C^{\prime}+4 \gamma(\varepsilon)\right)\left\|\sum_{k=1}^{n} f_{k}\right\|_{L_{1}}\right),
\end{aligned}
$$

o que prova o lema.

Lema 6.25 Dado $\varepsilon>0$, existe uma constante $C$ tal que se $\mathscr{T}$ é um semi-ideal de $L_{1}^{+}$e $\Phi$ é um funcional semilinear real definido em $\mathscr{T}$ que verifica para todo $f, g \in \mathscr{T}$, com $\operatorname{supp}(f) \cap$ $\operatorname{supp}(g)=\emptyset$, temos que $\Delta_{\Phi}(f, g) \geq 0$, então para todo $f_{1}, f_{2}, \ldots, f_{n} \in \mathscr{T}$,

$$
\Delta_{\Phi}\left(f_{1}, f_{2}, \ldots, f_{n}\right)+\delta \varepsilon \Delta_{\Lambda}\left(f_{1}, f_{2}, \ldots, f_{n}\right) \geq-C \delta \sum_{k=1}^{n}\left\|f_{k}\right\|_{L_{1}},
$$

onde $\delta=\delta(\Phi)$. 
Demonstração. Vamos provar o teorema para funções simples. Assuma que $f_{1}, f_{2}, \ldots, f_{n} \in$ $\mathscr{T}$ são funções simples e $f=f_{1}+f_{2}+\ldots+f_{n}$ com $\|f\|_{L_{1}}=1$. Seja $\Sigma_{0}$ o álgebra finita gerada por $f_{1}, f_{2}, \ldots, f_{n}$ (ver Definição 2.7). Definamos uma função $\phi: \Sigma_{0} \longrightarrow \mathbb{R}$ por $\phi(A)=\Phi\left(f \cdot 1_{A}\right)$. Então para $A, B$ disjuntos temos

$$
\begin{aligned}
\phi(A)+\phi(B)-\phi(A \cup B) & =\Phi\left(f \cdot 1_{A}\right)+\Phi\left(f \cdot 1_{B}\right)-\Phi\left(f \cdot 1_{A \cup B}\right) \\
& =\Phi\left(f \cdot 1_{A}\right)+\Phi\left(f \cdot 1_{B}\right)-\Phi\left(f \cdot 1_{A}+f \cdot 1_{B}\right) \\
& \leq\left|\Delta_{\Phi}\left(f \cdot 1_{A}, f \cdot 1_{B}\right)\right| \\
& \leq \delta\left(\left\|f \cdot 1_{A}\right\|_{L_{1}}+\left\|f \cdot 1_{B}\right\|_{L_{1}}\right) \\
& =\delta \int_{A \cup B} f d \mu
\end{aligned}
$$

e pela hipóteses do teorema, temos que $\Delta_{\Phi}\left(f 1_{A}, f 1_{B}\right) \geq 0$, isto é, $\phi(A \cup B) \leq \phi(A)+\phi(B)$. Resumindo

$$
\phi(A)+\phi(B)-\delta \int_{A \cup B} f d \mu \leq \phi(A \cup B) \leq \phi(A)+\phi(B) .
$$

Se $\mu_{0}(C)=\int_{C} \delta f d \mu$, então $\phi$ verifica as hipóteses do Teorema 6.22, logo existe uma medida com sinal $v$ definida na álgebra $\Sigma_{0}$ tal que $v(A) \leq \phi(A)$ para todo $A \in \Sigma_{0}$ e $v(S) \geq \phi(S)-4 \mu_{0}(S)=$ $\phi(S)-4 \delta=\Phi(f)-4 \delta$ (lembre-se que nós assumimos que $\|f\|_{L_{1}}=1$ ).

Por outro lado, seja $g$ uma função $\Sigma_{0}$ mensurável tal que $\operatorname{supp}(g) \subseteq \operatorname{supp}(f)$. Suponha que $\|g\|_{L_{1}}=1$ e definamos uma função $h$ por

$$
h(s)=\left\{\begin{array}{ll}
\frac{g(s)}{f(s)}, & \text { se } f(s) \neq 0 \\
0, & \text { se } f(s)=0
\end{array} .\right.
$$

Considere os conjuntos $\Sigma_{0}$ mensuráveis $A_{0}=\{s: h(s)<1\}$ e $A_{k}=\left\{s: 2^{k-1} \leq h(s)<2^{k}\right\}$ para $k>0$ natural. Como $\Sigma_{0}$ é um álgebra finita, temos que existe $N \in \mathbb{N}$ tal que $A_{k}=\emptyset$ para $k>N$. Então pelo Lema 6.24 temos que

$$
\Delta_{\Phi}\left(g \cdot 1_{A_{0}}, g \cdot 1_{A_{1}}, \ldots, g \cdot 1_{A_{N}}\right) \leq \delta\left(\varepsilon \log 2 \sum_{k=0}^{N}(k+1)\left\|g \cdot 1_{A_{k}}\right\|_{L_{1}}+C_{0}\right),
$$

onde $C_{0}$ depende de $\varepsilon$. Observe que 


$$
\begin{aligned}
\log 2 \sum_{k=0}^{N}(k+1)\left\|g \cdot 1_{A_{k}}\right\|_{L_{1}} & =\log 2 \int_{A_{0}} g d \mu+\log 2 \sum_{k=1}^{N}(k+1) \int_{A_{k}} g d \mu \\
& =\log 2 \int_{A_{0}} g d \mu+\sum_{k=1}^{N} \int_{A_{k}} g\left(\log 2^{k-1}+2 \log 2\right) d \mu \\
& \leq \log 2 \int_{A_{0}} g d \mu+\sum_{k=1}^{N} \int_{A_{k}} g \log h d \mu+2 \log 2 \sum_{k=1}^{N} \int_{A_{k}} g d \mu \\
& \leq \log 2 \int_{A_{0}} g d \mu+\int g \log h d \mu+2 \log 2 \\
& =\log 2 \int_{A_{0}} g d \mu+\int g \log h d \mu-\int g \log h d \mu+2 \log 2 \\
& =\log 2 \int_{A_{0}} g d \mu+\int g \log h d \mu+\int_{A_{0}} g \log \frac{f}{g} d \mu+2 \log 2 \\
& \leq \log 2+\int_{g} g \log h d \mu+\int_{A_{0}} g \cdot \frac{f}{g} d \mu+2 \log 2 \\
& \leq 3 \log 2+\int g \log h d \mu+1 .
\end{aligned}
$$

Portanto,

$$
\Delta_{\Phi}\left(g \cdot 1_{A_{0}}, g \cdot 1_{A_{1}}, \ldots, g \cdot 1_{A_{N}}\right) \leq \delta\left(\varepsilon \int g \log h d \mu+C_{1}\right),
$$

onde $C_{1}=\varepsilon(3 \log 2+1)+C_{0}$ (depende só de $\left.\varepsilon\right)$.

Por outro lado, para cada $k \in \mathbb{N}$, escrevemos

$$
h \cdot 1_{A_{k}}=2^{k} \sum_{j=1}^{\infty} 2^{-j} 1_{B_{k j}},
$$

onde os $B_{k j}$ são conjuntos de $\Sigma_{0}$ contidos em $A_{k}$. Seja $m \in \mathbb{N}$. Pelo Lema 6.24 temos que 


$$
\begin{aligned}
\Delta_{k m} & =\Delta_{\Phi}\left(2^{-1} f 1_{B_{k 1}}, 2^{-2} f 1_{B_{k 2}}, \ldots, 2^{-m} f 1_{B_{k m}}\right) \\
& \leq \delta\left(\sum_{j=1}^{m} j 2^{-j}\left\|f 1_{B_{k j}}\right\|_{L_{1}}+C_{2} \sum_{j=1}^{m} 2^{-j}\left\|f 1_{B_{k j}}\right\|_{L_{1}}\right) \\
& \leq \delta\left(\sum_{j=1}^{m} j 2^{-j}\left\|f 1_{A_{k}}\right\|_{L_{1}}+C_{2} \sum_{j=1}^{m} 2^{-j}\left\|f 1_{A_{k}}\right\|_{L_{1}}\right) \\
& \leq \delta C_{3}\left\|f 1_{A_{k}}\right\|_{L_{1}},
\end{aligned}
$$

onde $C_{2}$ depende só de $\varepsilon$ e $C_{3}=\sum_{j=1}^{\infty} j 2^{-j}+C_{2} \sum_{j=1}^{\infty} 2^{-j}$ depende de $\varepsilon$. Agora

$$
\begin{aligned}
\Phi\left(\sum_{j=1}^{m} 2^{k-j} f 1_{B_{k j}}\right) & =\sum_{j=1}^{m} \Phi\left(2^{k-j} f 1_{B_{k j}}\right)-\Delta_{\Phi}\left(2^{k-1} f 1_{B_{k 1}}, 2^{k-2} f 1_{B_{k 2}}, \ldots, 2^{k-m} f 1_{B_{k m}}\right) \\
& \geq \sum_{j=1}^{m} 2^{k-j} \phi\left(B_{k j}\right)-2^{k} C_{3} \delta\left\|f 1_{A_{k}}\right\|_{L_{1}} .
\end{aligned}
$$

Se $s \in A_{k}$, onde $k \geq 1$, então $2^{k-1} \leq g(s) / f(s)=h(s)$, portanto $-\left\|f 1_{A_{k}}\right\|_{L_{1}} \geq-\left\|g 1_{A_{k}}\right\|_{L_{1}}$, então

$$
\Phi\left(\sum_{j=1}^{m} 2^{k-j} f 1_{B_{k j}}\right) \geq \sum_{j=1}^{m} 2^{k-j} v\left(B_{k j}\right)-2 C_{3} \delta\left\|g 1_{A_{k}}\right\|_{L_{1}} .
$$

Agora pela definição de $h$, obtemos que $g \cdot 1_{A_{k}}=\sum_{j=1}^{\infty} 2^{k-j} f 1_{B_{k j}}$ e pelo Teorema 6.16 , temos que

$$
\Phi\left(\sum_{j=1}^{\infty} 2^{k-j} f 1_{B_{k j}}\right)=\Phi\left(g \cdot 1_{A_{k}}\right) \text {. }
$$

O Teorema da Convergência Dominada garante que

$$
\sum_{j=1}^{\infty} 2^{k-j} v\left(B_{k j}\right)=\sum_{j=1}^{\infty} \int 2^{k-j} 1_{B_{k j}} d v=\int 2^{k} \sum_{j=1}^{\infty} 2^{-j} 1_{B_{k j}} d v=\int_{A_{k}} h d v
$$

Se $m \rightarrow \infty$ na equação (6.38), então

$$
\Phi\left(g \cdot 1_{A_{k}}\right) \geq \int_{A_{k}} h d v-2 C_{3} \delta \int_{A_{k}} g d \mu, \text { se } k \geq 1 .
$$

Similarmente, obtemos que 


$$
\Phi\left(g \cdot 1_{A_{0}}\right) \geq \int_{A_{0}} h d v-2 C_{3} \delta \int_{A_{0}} f d \mu,
$$

somando as equações (6.39),(6.40), usando $\|f\|_{L_{1}}=\|g\|_{L_{1}}=1$ e o fato de $\left\{A_{k}: k \in \mathbb{N}\right\}$ ser uma coleção de conjuntos disjuntos

$$
\begin{aligned}
\sum_{k=0}^{N} \Phi\left(g \cdot 1_{A_{k}}\right) & \geq \sum_{k=0}^{N} \int_{A_{k}} h d v-2 C_{3} \delta \sum_{k=1}^{N} \int_{A_{k}} g d \mu-C_{3} \delta \int_{A_{0}} f d \mu \\
& \geq \int h d v-2 C_{3} \delta-C_{3} \delta \\
& =\int h d v-3 C_{3} \delta .
\end{aligned}
$$

Note que pela desigualdade 6.37 , temos que

$$
\begin{aligned}
\Phi(g) & =\Phi\left(\sum_{k=0}^{N} g \cdot 1_{A_{k}}\right) \\
& =\sum_{k=0}^{N} \Phi\left(g \cdot 1_{A_{k}}\right)-\Delta_{\Phi}\left(g \cdot 1_{A_{0}}, g \cdot 1_{A_{1}}, \ldots, g \cdot 1_{A_{N}}\right) \\
& \geq \int h d v-3 C_{3} \delta-\delta\left(\varepsilon \int g \log h d \mu+C_{1}\right) \\
& =\int h d v-\delta \varepsilon \int g \log h d \mu-\delta C_{4},
\end{aligned}
$$

onde $C_{4}$ é uma constante que depende só de $\varepsilon$, tendo assim o seguinte resultado:

$$
\Phi(g) \geq \int h d v-\delta \varepsilon \int g \log h d \mu-\delta C_{4},
$$

onde $g$ é uma função $\Sigma_{0}$-mensurável tal que $\|g\|_{L_{1}}=1$. Aplicando o resultado (6.41) para $g=$ $f_{k} /\left\|f_{k}\right\|_{L_{1}}$, temos que

$$
\Phi\left(f_{k}\right) \geq \int \frac{f_{k}}{f} d v-\delta \varepsilon \int g \log \frac{f_{k}}{f\left\|f_{k}\right\|_{L_{1}}} d \mu-\delta C_{4}\left\|f_{k}\right\|_{L_{1}},
$$

somando sobre $k$ 


$$
\begin{aligned}
\sum_{k=1}^{n} \Phi\left(f_{k}\right) & \geq \sum_{k=1}^{n} \int \frac{f_{k}}{f} d v-\delta \varepsilon \sum_{k=1}^{n} \int f_{k} \log \frac{f_{k}}{f\left\|f_{k}\right\|_{L_{1}}} d \mu-\delta C_{4} \sum_{k=1}^{n}\left\|f_{k}\right\|_{L_{1}} \\
& \geq v(S)-\delta \varepsilon \Delta_{\Lambda}\left(f_{1}, \ldots, f_{n}\right)-\delta C_{4} \\
& \geq \Phi(f)-4 \delta-\delta \varepsilon \Delta_{\Lambda}\left(f_{1}, \ldots, f_{n}\right)-\delta C_{4} \\
& =\Phi\left(\sum_{k=1}^{n} f_{k}\right)-\delta \varepsilon \Delta_{\Lambda}\left(f_{1}, \ldots, f_{n}\right)-\delta\left(C_{4}+4\right),
\end{aligned}
$$

concluindo assim que

$$
\Delta_{\Phi}\left(f_{1}, \ldots, f_{n}\right)+\delta \varepsilon \Delta_{\Lambda}\left(f_{1}, \ldots, f_{n}\right) \geq-C \delta,
$$

onde $C=C_{4}+4$ depende só de $\varepsilon$ e $f_{1}, \ldots, f_{n}$ são funções simples tais que $f=f_{1}+f_{2}+\ldots+f_{n}$ $\mathrm{e}\|f\|_{L_{1}}=1$.

Se $f_{1}, \ldots, f_{n}$ são funções simples arbitrárias, então definamos $f_{k}:=\frac{f_{k}}{\|f\|_{L_{1}}}$, assim

$$
\Delta_{\Phi}\left(f_{1}, \ldots, f_{n}\right)+\delta \varepsilon \Delta_{\Lambda}\left(f_{1}, \ldots, f_{n}\right) \geq-C \delta \sum_{k=1}^{n}\left\|f_{k}\right\|_{L_{1}},
$$

para $f_{1}, \ldots, f_{n}$ funções simples arbitrárias.

Se $f_{1}, \ldots, f_{n}$ são funções arbitrárias em $\mathscr{T}$, então para cada $f_{k}$, onde $1 \leq k \leq n$, existe uma sequência de funções simples não negativa e crescente $\left(f_{k, m}\right)_{m}$, com $f_{k, m} \leq f_{k}$ para todo $m \in \mathbb{N}$ (portanto, $\left.f_{k, m} \in \mathscr{T}\right)$ tal que $\left(f_{k, m}\right)_{m}$ converge q.s. para $f_{k}$. Aplicando o caso anterior, obtemos que

$$
\Delta_{\Phi}\left(f_{1, m}, \ldots, f_{n, m}\right)+\delta \varepsilon \Delta_{\Lambda}\left(f_{1, m}, \ldots, f_{n, m}\right) \geq-C \delta \sum_{k=1}^{n}\left\|f_{k, m}\right\|_{L_{1}},
$$

O Teorema da Convergência Dominada implica que $\lim _{m \rightarrow \infty}\left\|f_{k, m}-f_{k}\right\|_{L_{1}}=0$. Note que

$$
\begin{aligned}
\Delta_{\Phi}\left(f_{1, m}, \ldots, f_{n, m}\right) & =\sum_{k=1}^{n} \Phi\left(f_{k, m}\right)-\Phi\left(\sum_{k=1}^{n} f_{k, m}\right) \\
& =\sum_{k=1}^{n}\left(\Phi\left(f_{k, m}\right)-\Phi\left(f_{k}\right)\right)+\left(\Phi\left(\sum_{k=1}^{n} f_{k}\right)-\Phi\left(\sum_{k=1}^{n} f_{k, m}\right)\right)+\Delta_{\Phi}\left(f_{1}, \ldots, f_{n}\right) \\
& \leq \sum_{k=1}^{n}\left|\Phi\left(f_{k, m}\right)-\Phi\left(f_{k}\right)\right|+\left|\Phi\left(\sum_{k=1}^{n} f_{k}\right)-\Phi\left(\sum_{k=1}^{n} f_{k, m}\right)\right|+\Delta_{\Phi}\left(f_{1}, \ldots, f_{n}\right),
\end{aligned}
$$




\subsection{Aproximação de funcionais semilineares}

pelo Teorema 6.16, temos que

$$
\limsup _{m \rightarrow \infty} \Delta_{\Phi}\left(f_{1, m}, \ldots, f_{n, m}\right) \leq 2 \delta \sum_{k=1}^{n}\left\|f_{k}\right\|_{L_{1}}+2 \delta\left\|\sum_{k=1}^{n} f_{k}\right\|_{L_{1}}+\Delta_{\Phi}\left(f_{1}, \ldots, f_{n}\right) .
$$

Tomando limsup na equação (6.42), obtemos pela desigualdade acima e pelo fato de $\Lambda$ ser contínuo em $\left[0, f_{k}\right]$

$$
2 \delta \sum_{k=1}^{n}\left\|f_{k}\right\|_{L_{1}}+2 \delta\left\|\sum_{k=1}^{n} f_{k}\right\|_{L_{1}}+\Delta_{\Phi}\left(f_{1}, \ldots, f_{n}\right)+\delta \varepsilon \Delta_{\Lambda}\left(f_{1}, \ldots, f_{n}\right) \geq-C \delta \sum_{k=1}^{n}\left\|f_{k}\right\|_{L_{1}},
$$

$\operatorname{logo}$

$$
\Delta_{\Phi}\left(f_{1}, \ldots, f_{n}\right)+\delta \varepsilon \Delta_{\Lambda}\left(f_{1}, \ldots, f_{n}\right)+\geq-(C+4) \delta \sum_{k=1}^{n}\left\|f_{k}\right\|_{L_{1}} .
$$

para todo $f_{1}, \ldots, f_{n} \in \mathscr{T}$ arbitrários.

Teorema 6.26 Dado $\varepsilon>0$, existe uma constante $C$ (que depende de $\varepsilon$ ) tal que para todo semi-ideal estrito $\mathscr{T}$ contido em $(L \log L)_{+}$e todo funcional semilinear $\Phi: \mathscr{T} \longrightarrow \mathbb{R}$, com $\delta(\Phi) \leq(1-\varepsilon) \log 2$, temos que existe um espaço de Köthe $X$ tal que

$$
d\left(\Phi, \Phi_{X}-\Phi_{X^{*}}\right) \leq C
$$

Demonstração. Seja $0<\varepsilon<1$. Aplicando o Lema 6.23 para $\sigma=\frac{2-\varepsilon}{2(1-\varepsilon)}>1$ e $\alpha=1-\varepsilon$, temos que existe uma constante $C_{0}$, que depende de $\varepsilon$, tal que para todo semi-ideal estrito $\mathscr{T}$ contido em $(L \log L)_{+}$e todo funcional semilinear $\Phi: \mathscr{T} \longrightarrow \mathbb{R}$ que satisfaz

$$
\Delta_{\Phi}(f, g) \leq(1-\varepsilon) \log 2\left(\|f\|_{L_{1}}+\|g\|_{L_{1}}\right),
$$

obtemos que

$$
\left|\Delta_{\Phi}\left(f_{1}, \ldots, f_{n}\right)\right| \leq\left(1-\frac{1}{2} \varepsilon\right) \sum_{k=1}^{n}\left\|f_{k}\right\|_{L_{1}} \log \frac{\|f\|_{L_{1}}}{\left\|f_{k}\right\|_{L_{1}}}+C_{0}\|f\|_{L_{1}},
$$

para $f_{1}, \ldots, f_{n} \in \mathscr{T}$ e $f=\sum_{k=1}^{n} f_{k}$.

Se $f_{1}, \ldots, f_{n} \in \mathscr{T}$ são disjuntos e $f=\sum_{k=1}^{n} f_{k}$, então

$$
\Delta_{\Lambda}\left(f_{1}, \ldots, f_{n}\right)=\sum_{k=1}^{n}\left\|f_{k}\right\|_{L_{1}} \log \frac{\|f\|_{L_{1}}}{\left\|f_{k}\right\|_{L_{1}}}
$$

portanto, 


$$
\left|\Delta_{\Phi}\left(f_{1}, \ldots, f_{n}\right)\right| \leq\left(1-\frac{1}{2} \varepsilon\right) \Delta_{\Lambda}\left(f_{1}, \ldots, f_{n}\right)+C_{0}\|f\|_{L_{1}},
$$

para $f_{1}, \ldots, f_{n} \in \mathscr{T}$ disjuntos e $f=\sum_{k=1}^{n} f_{k}$.

Seja $\Psi_{0}=\Phi+\left(1-\frac{1}{2} \varepsilon\right) \Lambda$. Para $f_{1}, \ldots, f_{n} \in \mathscr{T}$ disjuntos, deduzimos pela desigualdade (6.43) que

$$
\begin{aligned}
\Delta_{\Psi_{0}}\left(f_{1}, \ldots, f_{n}\right) & =\Delta_{\Phi}\left(f_{1}, \ldots, f_{n}\right)+\left(1-\frac{1}{2} \varepsilon\right) \Delta_{\Lambda}\left(f_{1}, \ldots, f_{n}\right) \\
& \geq-\left(1-\frac{1}{2} \varepsilon\right) \Delta_{\Lambda}\left(f_{1}, \ldots, f_{n}\right)-C_{0} \sum_{k=1}^{n}\left\|f_{k}\right\|_{L_{1}}+\left(1-\frac{1}{2} \varepsilon\right) \Delta_{\Lambda}\left(f_{1}, \ldots, f_{n}\right) \\
& =-C_{0} \sum_{k=1}^{n}\left\|f_{k}\right\|_{L_{1}} .
\end{aligned}
$$

Definamos como $\Psi(f)=\inf \sum_{k=1}^{n} \Psi_{0}\left(f_{k}\right)$, onde o ínfimo é tomado no conjunto dos $f_{1}, \ldots, f_{n} \in$ $\mathscr{T}$ disjuntos tais que $f=\sum_{k=1}^{n} f_{k}$. Agora

$$
\begin{aligned}
\Psi_{0}(f)-C_{0}\|f\|_{L_{1}} & =\sum_{k=1}^{n} \Psi_{0}\left(f_{k}\right)-\Delta_{\Psi_{0}}\left(f_{1}, \ldots, f_{n}\right)-C_{0}\|f\|_{L_{1}} \\
& \leq \sum_{k=1}^{n} \Psi_{0}\left(f_{k}\right)+C_{0} \sum_{k=1}^{n}\left\|f_{k}\right\|_{L_{1}}-C_{0}\|f\|_{L_{1}} \\
& =\sum_{k=1}^{n} \Psi_{0}\left(f_{k}\right)
\end{aligned}
$$

para todo $f_{1}, \ldots, f_{n} \in \mathscr{T}$ disjuntos, com $f=\sum_{k=1}^{n} f_{k}, \operatorname{logo} \Psi_{0}(f)-C_{0}\|f\|_{L_{1}} \leq \Psi(f)$. Pela definição de $\Psi$, obtemos que $\Psi(f) \leq \Psi_{0}(f)$, resumindo

$$
0 \leq \Psi_{0}(f)-\Psi(f) \leq C_{0}\|f\|_{L_{1}} .
$$

Note que 


$$
\begin{aligned}
\left|\Delta_{\Psi}\left(f_{1}, \ldots, f_{n}\right)-\Delta_{\Psi_{0}}\left(f_{1}, \ldots, f_{n}\right)\right| & =\left|\sum_{k=1}^{n}\left(\Psi_{0}\left(f_{k}\right)-\Psi\left(f_{k}\right)\right)+\left(\Psi(f)-\Psi_{0}(f)\right)\right| \\
& \leq C_{0} \sum_{k=1}^{n}\left\|f_{k}\right\|_{L_{1}}+C_{0}\|f\|_{L_{1}} \\
& =2 C_{0} \sum_{k=1}^{n}\left\|f_{k}\right\|_{L_{1}},
\end{aligned}
$$

isto é,

$$
\left|\Delta_{\Psi}\left(f_{1}, \ldots, f_{n}\right)-\Delta_{\Psi_{0}}\left(f_{1}, \ldots, f_{n}\right)\right| \leq 2 C_{0} \sum_{k=1}^{n}\left\|f_{k}\right\|_{L_{1}} .
$$

Afirmamos que $\Delta_{\Psi}(f, g) \geq 0$ para todo $f, g \in \mathscr{T}$ disjuntos. De fato, sejam $f=\sum_{k} f_{k}$ e $g=\sum g_{k}$, onde os $f_{k}$ são disjuntos e os $g_{s}$ são disjuntos. Como $f, g$ são disjuntos, temos que os $f_{k}, g_{s}$ são disjuntos. Agora $\Psi(f+g) \leq \sum_{k} \Phi\left(f_{k}\right)+\sum_{s} \Phi\left(f_{s}\right)$, assim $\Psi(f+g) \leq \Psi(f)+\Psi(g)$, o que prova $\Delta_{\Psi}(f, g) \geq 0$, sempre que $f, g$ sejam disjuntas. Note que $\Psi$ verifica as hipóteses do Lema 6.25, portanto, para $\frac{\varepsilon}{2 \delta(\Psi)}$, existe $C_{0}$ (que depende de $\varepsilon$ ) tal que

$$
\Delta_{\Psi}\left(f_{1}, \ldots, f_{n}\right)+\delta(\Psi) \cdot \frac{\varepsilon}{2 \delta(\Psi)} \Delta_{\Lambda}\left(f_{1}, \ldots, f_{n}\right) \geq-C_{0} \cdot \delta(\Psi) \sum_{k=1}^{n}\left\|f_{k}\right\|_{L_{1}},
$$

para todo $f_{1}, \ldots, f_{n} \in \mathscr{T}$, que equivale a

$$
\Delta_{\Psi}\left(f_{1}, \ldots, f_{n}\right) \geq-\frac{\varepsilon}{2} \Delta_{\Lambda}\left(f_{1}, \ldots, f_{n}\right)-C_{0} \sum_{k=1}^{n}\left\|f_{k}\right\|_{L_{1}},
$$

para todo $f_{1}, \ldots, f_{n} \in \mathscr{T}$, onde $C_{0}:=\delta(\Phi) \cdot C_{0}$. Usando esta desigualdade e (6.44), demonstramos que

$$
\Delta_{\Psi_{0}}\left(f_{1}, \ldots, f_{n}\right) \geq-\frac{\varepsilon}{2} \Delta_{\Lambda}\left(f_{1}, \ldots, f_{n}\right)-C_{1} \sum_{k=1}^{n}\left\|f_{k}\right\|_{L_{1}},
$$

onde $C_{1}$ depende de $\varepsilon$. Como $\Psi_{0}=\Phi+\left(1-\frac{1}{2} \varepsilon\right) \Lambda$, temos que

$$
\Delta_{\Phi}\left(f_{1}, \ldots, f_{n}\right) \geq-\Delta_{\Lambda}\left(f_{1}, \ldots, f_{n}\right)-C_{2} \sum_{k=1}^{n}\left\|f_{k}\right\|_{L_{1}},
$$

onde $C_{2}$ depende de $\varepsilon$.

Analogamente provamos para $-\Phi$ que 


$$
\Delta_{-\Phi}\left(f_{1}, \ldots, f_{n}\right) \geq-\Delta_{\Lambda}\left(f_{1}, \ldots, f_{n}\right)-C_{2} \sum_{k=1}^{n}\left\|f_{k}\right\|_{L_{1}}
$$

Resumindo,

$$
\left|\Delta_{\Phi}\left(f_{1}, \ldots, f_{n}\right)\right| \leq \Delta_{\Lambda}\left(f_{1}, \ldots, f_{n}\right)+C_{2} \sum_{k=1}^{n}\left\|f_{k}\right\|_{L_{1}},
$$

para todo $f_{1}, \ldots, f_{n} \in \mathscr{T}$.

O funcional semilinear $\frac{1}{2}(\Phi+\Lambda)$ satisfaz as condições da Proposição 6.21, pois a desigualdade (6.45) equivale a

$$
-\frac{1}{2} C_{2} \sum_{k=1}^{n}\left\|f_{k}\right\|_{L_{1}} \leq \Delta_{\frac{\Phi+\Lambda}{2}}\left(f_{1}, \ldots, f_{n}\right) \leq \Delta_{\Lambda}\left(f_{1}, \ldots, f_{n}\right)+\frac{1}{2} C_{2} \sum_{k=1}^{n}\left\|f_{k}\right\|_{L_{1}}
$$

para todo $f_{1}, \ldots, f_{n} \in \mathscr{T}$. A Proposição 6.21 implica que existe um espaço de Köthe $X$ tal que

$$
d\left(\frac{\Phi+\Lambda}{2}, \Phi_{X}\right) \leq C_{3},
$$

onde $C_{3}$ depende de $\varepsilon$, assim

$$
\begin{aligned}
C_{3} & \geq d\left(\frac{\Phi+\Lambda}{2}, \Phi_{X}\right) \\
& =\sup _{\|f\|_{L_{1}} \leq 1}\left|\frac{\Phi(f)+\Lambda(f)}{2}-\Phi_{X}(f)\right| \\
& =\sup _{\|f\|_{L_{1}} \leq 1}\left|\frac{\Phi(f)-\Phi_{X}(f)+\Lambda(f)-\Phi_{X}(f)}{2}\right| \\
& =\frac{1}{2} \cdot \sup _{\|f\|_{L_{1}} \leq 1}\left|\Phi(f)-\Phi_{X}(f)+\Phi_{X^{*}}(f)\right| \\
& =\frac{1}{2} \cdot d\left(\Phi, \Phi_{X}-\Phi_{X^{*}}\right) .
\end{aligned}
$$

Portanto, existe $C$ que depende de $\varepsilon$ tal que $d\left(\Phi, \Phi_{X}-\Phi_{X^{*}}\right) \leq C$.

A prova do seguinte resultado é imediata pelo teorema acima. 


\subsection{Aproximação de funcionais semilineares}

Teorema 6.27 Dado $\varepsilon>0$, existe uma constante $C$ (que depende de $\varepsilon$ ) tal que para todo semiideal estrito $\mathscr{T}$ de $L_{1}^{+}$e todo funcional semilinear $\Phi: \mathscr{T} \longrightarrow \mathbb{R}, \operatorname{com} \delta(\Phi) \leq(1-\varepsilon) \log 2$, temos que existe um espaço de Köthe $X$ tal que

$$
\left|\Phi(f)-\left(\Phi_{X}(f)-\Phi_{X^{*}}(f)\right)\right| \leq C\|f\|_{L_{1}},
$$

para todo $f \in \mathscr{T}$. 
$\prod_{\text {Capítulo }}$

\section{Centralizadores em espaços de Köthe}

Neste capítulo vamos estudar um tipo de aplicações quase-lineares chamados de centralizadores. O objetivo deste capítulo é caracterizar as auto-extensões dos espaços de Köthe interpolados.

Definição 7.1 (Centralizador) Seja X um Espaços de Köthe. Um centralizador em X é uma função $\Omega: X \rightarrow L_{0}$ tal que

- $\Omega(\alpha x)=\alpha \Omega(x)$ para todo $x \in X$ e todo $\alpha \in \mathbb{C}$.

- Existe uma constante $\rho(\Omega)>0$ tal que

$$
\|\Omega(u x)-u \Omega(x)\|_{X} \leq \rho(\Omega)\|u\|_{\infty}\|x\|_{X} .
$$

para todo $u \in L_{\infty}$ e todo $x \in X$.

Vamos denotar como $\rho(\Omega)$ a menor constante que satisfaz essa condição . O centralizador $\Omega$ é dito Real se $\Omega(x)$ é uma função de valor real sempre que x seja uma função de valor real.

\subsection{Propriedades dos centralizadores}

Proposição 7.2 Seja $\Omega$ um centralizador definido em $X$ com

$$
\|\Omega(u x)-u \Omega(x)\|_{X} \leq \rho\|u\|_{\infty}\|x\|_{X}
$$

para todo $x \in X$ e $u \in L_{\infty}$, onde $\rho:=\rho(\Omega)$. Então

$$
\|\Omega(x+y)-\Omega(x)-\Omega(y)\|_{X} \leq 3 \rho\left(\|x\|_{X}+\|y\|_{X}\right),
$$

ou seja, todo centralizador em X é uma função quase-linear em X. 


\subsection{Propriedades dos centralizadores}

Demonstração. Seja $z=|x|+|y|$. Definimos $u=\frac{x}{z}$ e $v=\frac{y}{z}$.

Note que $|u(s)|,|v(s)| \leq 1$ para quase todo $s \in S$ e também que $|u(s)+v(s)| \leq 1$ para quase todo $s \in S$, o que prova $u, v, u+v \in L_{\infty} \mathrm{e}\|u\|_{\infty},\|v\|_{\infty},\|u+v\|_{\infty} \leq 1$. Escrevamos $x=u z$ e $y=v z$, assim

$$
\begin{aligned}
\Omega(x+y)-\Omega(x)-\Omega(y)= & \Omega((u+v) z)-\Omega(u z)-\Omega(v z) \\
= & {[\Omega((u+v) z)-(u+v) \Omega(z)]+[u \Omega(z)-\Omega(u z)] } \\
& +[v \Omega(z)-\Omega(v z)],
\end{aligned}
$$

onde cada termo acima está em $X$ e em consequentemente

$$
\begin{aligned}
\|\Omega(x+y)-\Omega(x)-\Omega(y)\|_{X} \leq & \|\Omega((u+v) z)-(u+v) \Omega(z)\|_{X}+\|u \Omega(z)-\Omega(u z)\|_{X} \\
& +\|v \Omega(z)-\Omega(v z)\|_{X} \\
\leq & \rho\|u+v\|_{\infty}\|z\|_{X}+\rho\|u\|_{\infty}\|z\|_{X}+\rho\|v\|_{\infty}\|z\|_{X} \\
\leq & 3 \rho\|z\|_{X} \\
= & 3 \rho\||x|+|y|\|_{X} \\
\leq & 3 \rho\left(\|x\|_{X}+\|y\|_{X}\right) .
\end{aligned}
$$

Definição 7.3 Dois centralizadores $\Omega, \Omega^{\prime}$ em $X$ são limitadamente equivalentes, se $\Omega(x)-$ $\Omega^{\prime}(x) \in X$ para todo $x \in X$ e se existe uma constante $C$ tal que

$$
\left\|\Omega(x)-\Omega^{\prime}(x)\right\|_{X} \leq C\|x\|_{X}
$$

para todo $x \in X$.

Definição 7.4 (Espaço derivado) $O$ espaço derivado associado a um centralizador $\Omega: X \longrightarrow$ $L_{0}$, é definido por

$$
d_{\Omega} X=\left\{(x, y) \in X \times L_{0}: \Omega(x)-y \in X\right\},
$$

com a quase-norma $\|(x, y)\|_{d_{\Omega} X}=\|x\|_{X}+\|\Omega(x)-y\|_{X}$. 


\subsection{Propriedades dos centralizadores}

Observação 7.5 Pela Proposição 7.2, temos que todo centralizador $\Omega: X \longrightarrow L_{0}$ é uma aplicação quase-linear, portanto, a Proposição 3.9 garante que o espaço $d_{\Omega} X$ é uma soma torcida.

Se $\Omega^{\prime}: X \longrightarrow L_{0}$ é um centralizador limitadamente equivalente a $\Omega$, então a Proposição 3.13, garante que os espaços derivados $d_{\Omega} X$ e $d_{\Omega^{\prime}} X$ são somas torcidas equivalentes.

Agora mostraremos que todo centralizador Real pode ser aproximado de uma certa maneira por um centralizador em $L_{1}$.

Proposição 7.6 Seja $\Omega$ um centralizador real em $X$ tal que

$$
\|\Omega(u x)-u \Omega(x)\|_{X} \leq \rho(\Omega)\|u\|_{\infty}\|x\|_{X},
$$

para todo $x \in X$ e $u \in L_{\infty}$. Então existe um centralizador real $\Omega^{1}$ em $L_{1}$ tal que

$$
\left\|\Omega^{1}\left(y y^{*}\right)-y^{*} \Omega(y)\right\|_{L_{1}} \leq K\|y\|_{X}\left\|y^{*}\right\|_{X^{*}}
$$

para todo y $\in X$ e todo $y^{*} \in X^{*}$, onde $K=4 \rho(\Omega)$. Além disso, $\Omega^{1}$ é único no seguinte sentido: se $\Omega_{0}^{1}$ é outro centralizador em $L_{1}$ que satisfaz a desigualdade (7.3), então $\Omega_{0}^{1}$ e $\Omega^{1}$ são centralizadores limitadamente equivalentes em $L_{1}$.

Demonstração. Denotemos por $\rho:=\rho(\Omega)$. Suponha $x_{1}, x_{2} \in X$ e $y_{1}, y_{2} \in X^{*} \operatorname{com} f=$ $x_{1} \cdot y_{1}=x_{2} \cdot y_{2}$. Note que que $F=\left|x_{1}\right|+\left|x_{2}\right| \in X$. Definamos $F^{-1}$ como

$$
F^{-1}(s)= \begin{cases}\frac{1}{\left|x_{1}(s)\right|+\left|x_{2}(s)\right|} & \text { se } x_{1}(s) \neq 0 \text { ou } x_{2}(s) \neq 0, \\ 0 & \text { se } x_{1}(s)=0 \text { e } x_{2}(s)=0 .\end{cases}
$$

Observe que $x_{1} F^{-1} \in L_{\infty}$, com $\left\|x_{1} F^{-1}\right\|_{\infty} \leq 1$, assim

$$
\begin{aligned}
\left\|\Omega\left(x_{1}\right)-x_{1} F^{-1} \Omega(F)\right\|_{X} & \leq \rho\left\|x_{1} F^{-1}\right\|_{\infty}\|F\|_{X} \\
& \leq \rho\|F\|_{X} .
\end{aligned}
$$

O fato $y_{1} \in X^{*}$ implica que $y_{1}\left(\Omega x_{1}-x_{1} F^{-1} \Omega F\right) \in L_{1}$ e

$$
\begin{aligned}
\left\|y_{1}\left(\Omega x_{1}-x_{1} F^{-1} \Omega F\right)\right\|_{L_{1}} & \leq\left\|y_{1}\right\|_{X^{*}}\left\|\Omega x_{1}-x_{1} F^{-1} \Omega F\right\|_{X} \\
& \leq \rho\left\|y_{1}\right\|_{X^{*}}\|F\|_{X} \\
& \leq \rho\left\|y_{1}\right\|_{X^{*}}\left(\left\|x_{1}\right\|_{X}+\left\|x_{2}\right\|_{X}\right),
\end{aligned}
$$




\subsection{Propriedades dos centralizadores}

isto mostra que

$$
\left\|y_{1} \Omega x_{1}-f F^{-1} \Omega F\right\|_{L_{1}} \leq \rho\left\|y_{1}\right\|_{X^{*}}\left(\left\|x_{1}\right\|_{X}+\left\|x_{2}\right\|_{X}\right) .
$$

Similarmente para $y_{2} \in X^{*}$ obtemos que

$$
\left\|y_{2} \Omega x_{2}-f F^{-1} \Omega F\right\|_{L_{1}} \leq \rho\left\|y_{2}\right\|_{X^{*}}\left(\left\|x_{1}\right\|_{X}+\left\|x_{2}\right\|_{X}\right) .
$$

Somando as últimas desigualdades e usando desigualdade triangular, segue-se que

$$
\left\|y_{1} \Omega x_{1}-y_{2} \Omega x_{2}\right\|_{L_{1}} \leq \rho\left(\left\|x_{1}\right\|_{X}+\left\|x_{2}\right\|_{X}\right)\left(\left\|y_{1}\right\|_{X^{*}}+\left\|y_{2}\right\|_{X^{*}}\right)
$$

Suponha que $x_{1}, x_{2}, y_{1}, y_{2}$ são não nulos, portanto,

$$
\alpha=\frac{\left\|x_{1}\right\|_{X}^{1 / 2}\left\|y_{2}\right\|_{X^{*}}^{1 / 2}}{\left\|x_{2}\right\|_{X}^{1 / 2}\left\|y_{1}\right\|_{X^{*}}^{1 / 2}}>0 .
$$

Substituindo $x_{2}$ e $y_{2}$ por $\alpha x_{2}$ e $\alpha^{-1} y_{2}$, em (7.4), respectivamente, deduzimos que

$$
\left\|y_{1} \Omega x_{1}-y_{2} \Omega x_{2}\right\|_{L_{1}} \leq \rho\left(\left\|x_{1}\right\|_{X}^{1 / 2}\left\|y_{1}\right\|_{X^{*}}^{1 / 2}+\left\|x_{2}\right\|_{X}^{1 / 2}\left\|y_{2}\right\|_{X^{*}}^{1 / 2}\right)^{2}
$$

para todo $x_{1}, x_{2} \in X$ e todo $y_{1}, y_{2} \in X^{*}$ tais que $f=x_{1} \cdot y_{1}=x_{2} \cdot y_{2}$.

Se $f \in L_{1}$, então pela fatoração de Lozanovskii, existe um únicos par $\left(x, x^{*}\right) \in X \times X^{*}$ tal que $x \geq 0,\|x\|_{X}=1,\left\|x^{*}\right\|_{X^{*}}=\|f\|_{L_{1}}, \operatorname{supp}(x)=\operatorname{supp}\left(x^{*}\right)=\operatorname{supp}(f)$ e $f=x \cdot x^{*}$.

Definamos $\Omega^{1}: L_{1} \longrightarrow L_{0}$ por $\Omega^{1}(f)=x^{*} \Omega(x)$.

Vejamos que $\Omega^{1}$ é um centralizador. De fato, note que $\Omega^{1}$ é homogênea.

Afirmamos que $\left\|\Omega^{1}(u f)-u \Omega^{1}(f)\right\|_{L_{1}} \leq 5 \rho\|u\|_{\infty}\|f\|_{L_{1}}$, para todo $u \in L_{\infty}$ e todo $f \in L_{1}$. De fato, sejam $u f=y \cdot y^{*}$ e $f=x \cdot x^{*}$ as fatorações de Lozanovskii de $u f$ e $f$, respectivamente. Observe que $u f=y \cdot y^{*}=(u x) \cdot x^{*}$. Pela desigualdade (7.5), com $y_{1}=y^{*}, x_{1}=y, y_{2}=x^{*}, x_{2}=u x$, temos que $y^{*} \Omega(y)-x^{*} \Omega(u x) \in L_{1} \mathrm{e}$

$$
\begin{aligned}
\left\|y^{*} \Omega(y)-x^{*} \Omega(u x)\right\|_{L_{1}} & \leq \rho\left(\|y\|_{X}^{1 / 2}\left\|y^{*}\right\|_{X^{*}}^{1 / 2}+\|u x\|_{X}^{1 / 2}\left\|x^{*}\right\|_{X^{*}}^{1 / 2}\right)^{2} \\
& =\rho\left(\|u f\|_{L_{1}}^{1 / 2}+\|u x\|_{X}^{1 / 2}\|f\|_{L_{1}}^{1 / 2}\right)^{2} \\
& \leq \rho\left(\left(\|u\|_{\infty}\|f\|_{L_{1}}\right)^{1 / 2}+\left(\|u\|_{\infty}\|x\|_{X}\|f\|_{L_{1}}\right)^{1 / 2}\right)^{2} \\
& =4 \rho\|u\|_{\infty}\|f\|_{L_{1}} .
\end{aligned}
$$

Mas ainda 


\subsection{Propriedades dos centralizadores}

$$
\begin{aligned}
\Omega^{1}(u f)-u \Omega^{1}(f) & =y^{*} \Omega(y)-u x^{*} \Omega(x) \\
& =\left(y^{*} \Omega(y)-x^{*} \Omega(u x)\right)+\left(x^{*}(\Omega(u x)-u \Omega(x))\right) \in L_{1}
\end{aligned}
$$

A desigualdade triangular implica

$$
\begin{aligned}
\left\|\Omega^{1}(u f)-u \Omega^{1}(f)\right\|_{L_{1}} & \leq\left\|y^{*} \Omega(y)-x^{*} \Omega(u x)\right\|_{L_{1}}+\left\|x^{*}(\Omega(u x)-u \Omega(x))\right\|_{L_{1}} \\
& \leq 4 \rho\|u\|_{\infty}\|f\|_{L_{1}}+\rho\left\|x^{*}\right\|_{X^{*}}\|u\|_{\infty}\|x\|_{X} \\
& =5 \rho\|u\|_{\infty}\|f\|_{L_{1}} .
\end{aligned}
$$

Consequentemente, $\Omega^{1}$ é um centralizador sobre $L_{1}$. Vejamos que $\Omega^{1}$ verifica a condição (7.3). De fato, seja $y \cdot y^{*}=x \cdot x^{*}$, onde $x \cdot x^{*}$ é a fatoração de Lozanovskii de $y \cdot y^{*}$, isto é, $\|x\|_{X}=$ $1,\left\|x^{*}\right\|_{X^{*}}=\left\|y \cdot y^{*}\right\|_{L_{1}}, x \geq 0, \operatorname{supp}(x)=\operatorname{supp}\left(x^{*}\right)=\operatorname{supp}\left(y \cdot y^{*}\right)$. Se $y_{1}=y^{*}, x_{1}=y, y_{2}=x^{*} \mathrm{e}$ $x_{2}=x$ na desigualdade (7.5), obtemos que

$$
\begin{aligned}
\left\|y^{*} \Omega(y)-x^{*} \Omega(x)\right\|_{L_{1}} & \leq \rho(\Omega)\left(\|y\|_{X}^{1 / 2}\left\|y^{*}\right\|_{X^{*}}^{1 / 2}+\|x\|_{X}^{1 / 2}\left\|x^{*}\right\|_{X^{*}}^{1 / 2}\right)^{2} \\
& \leq 4 \rho(\Omega)\|y\|_{X}\left\|y^{*}\right\|_{X^{*}}
\end{aligned}
$$

pela definição de $\Omega^{1}$, concluimos que

$$
\left\|\Omega^{1}\left(y y^{*}\right)-y^{*} \Omega(y)\right\|_{L_{1}} \leq 4 \rho(\Omega)\|y\|_{X}\left\|y^{*}\right\|_{X^{*}}
$$

para todo $y \in X$ e todo $y^{*} \in X^{*}$.

Agora vejamos que se $\Omega_{0}^{1}$ é outro centralizador em $L_{1}$ que satisfaz

$$
\left\|\Omega_{0}^{1}\left(y \cdot y^{\star}\right)-y^{*} \Omega_{0}^{1}(y)\right\|_{L_{1}} \leq K_{0}\|y\|_{X}\|y\|_{X^{*}}
$$

para todo $y \in X$ e todo $y^{*} \in X^{*}$ e alguma constante $K_{0}>0$, então $\Omega^{1}$ é limitadamente equivalente a $\Omega_{0}^{1}$. Seja $f=x \cdot x^{*} \in L_{1}$ a fatoração de Lozanovskii, isto é, $\|x\|_{X}=1,\left\|x^{*}\right\|_{X^{*}}=\|f\|_{L_{1}}, x \geq$ $0, \operatorname{supp}(x)=\operatorname{supp}\left(x^{*}\right)=\operatorname{supp}(f)$. Note que 


\subsection{Propriedades dos centralizadores}

$$
\begin{aligned}
\left\|\Omega^{1}(f)-\Omega_{0}^{1}(f)\right\|_{L_{1}} & =\left\|\Omega^{1}\left(x \cdot x^{*}\right)-\Omega_{0}^{1}\left(x \cdot x^{*}\right)\right\|_{L_{1}} \\
& \leq\left\|\Omega^{1}\left(x \cdot x^{*}\right)-x^{*} \Omega(x)\right\|_{L_{1}}+\left\|x^{*} \Omega(x)-\Omega_{0}^{1}\left(x \cdot x^{*}\right)\right\|_{L_{1}} \\
& \leq\left(K+K_{0}\right)\|x\|_{X}\left\|x^{*}\right\|_{X^{*}} \\
& =\left(K+K_{0}\right)\|f\|_{L_{1}},
\end{aligned}
$$

ou seja, $\Omega^{1}$ e $\Omega_{0}^{1}$ são centralizadores limitadamente equivalentes.

Observação 7.7 O Teorema 7.6 mostra que se $\Omega$ é um centralizador tal que

$$
\|\Omega(u x)-u \Omega(x)\|_{X} \leq \rho(\Omega)\|u\|_{\infty}\|x\|_{X},
$$

para todo $x \in X$ e $u \in L_{\infty}$, então $\Omega^{1}$ verifica

$$
\left\|u \Omega^{1}(f)-\Omega(u f)\right\|_{L_{1}} \leq 5 \rho(\Omega)\|u\|_{\infty}\|f\|_{L_{1}}
$$

para todo $u \in L_{\infty}$ e todo $f \in L_{1}$.

Corolário 7.8 Se $\Omega$ e $\Omega_{0}$ são centralizadores limitadamente equivalentes de X, então $\Omega^{1}$ e $\Omega_{0}^{1}$ são centralizadores limitadamente equivalentes de $L_{1}$.

Demonstração. Como $\Omega$ e $\Omega_{0}$ são centralizadores limitadamente equivalentes de $X$, então existe $C>0$, tal que

$$
\left\|\Omega(x)-\Omega_{0}(x)\right\|_{X} \leq C\|x\|
$$

para todo $x \in X$. Agora seja $f=x \cdot x^{*} \in L_{1}$ a fatoração de Lozanovskii, isto é, $\|x\|_{X}=1,\left\|x^{*}\right\|_{X^{*}}=$ $\|f\|_{L_{1}}, x \geq 0, \operatorname{supp}(x)=\operatorname{supp}\left(x^{*}\right)=\operatorname{supp}(f)$. Então

$$
\begin{aligned}
\left\|\Omega^{1}(f)-\Omega_{0}^{1}(f)\right\|_{L_{1}}= & \left\|\Omega^{1}\left(x \cdot x^{*}\right)-\Omega_{0}^{1}\left(x \cdot x^{*}\right)\right\|_{L_{1}} \\
\leq & \left\|\Omega^{1}\left(x \cdot x^{*}\right)-x^{*} \Omega(x)\right\|_{L_{1}}+\left\|x^{*} \Omega(x)-x^{*} \Omega_{0}(x)\right\|_{L_{1}} \\
& +\left\|x^{*} \Omega_{0}(x)-\Omega_{0}^{1}\left(x \cdot x^{*}\right)\right\|_{L_{1}} \\
\leq & 4 \rho(\Omega)\|x\|_{X}\left\|x^{*}\right\|_{X^{*}}+C\|x\|_{X}\left\|x^{*}\right\|_{X^{*}}+4 \rho\left(\Omega_{0}\right)\|x\|_{X}\left\|x^{*}\right\|_{X^{*}} \\
= & C_{1}\|x\|_{X}\left\|x^{*}\right\|_{X^{*}} \\
= & C_{1}\|f\|_{L_{1}},
\end{aligned}
$$


onde $C_{1}=4 \rho\left(\Omega_{0}\right)+4 \rho(\Omega)+C$.

Corolário 7.9 Se $\Omega$ é um centralizador tal que

$$
\|\Omega(u x)-u \Omega(x)\|_{X} \leq \rho(\Omega)\|u\|_{\infty}\|x\|_{X},
$$

para todo $x \in X$ e $u \in L_{\infty}$, então $\Omega^{1}$ verifica

$$
\left\|\Omega^{1}(f+g)-\Omega^{1}(f)-\Omega^{1}(g)\right\|_{L_{1}} \leq 15 \rho(\Omega)\left(\|f\|_{L_{1}}+\|g\|_{L_{1}}\right)
$$

para todo $f, g \in L_{1}$.

Demonstração. É uma consequência imediata da Observação 7.7 e da Proposição 7.2.

\subsection{Auto-extensões de espaços interpolados}

Sejam $X_{0}, X_{1}$ espaços de Köthe e $X=X_{\theta}=\left[X_{0}, X_{1}\right]_{\theta}$. Definimos um espaço vetorial $d X_{\theta}$ como

$$
d X_{\theta}=\left\{(x, y) \in L_{0} \times L_{0}: x=F(\theta)=x, F^{\prime}(\theta)=y \text { para algum } F \in \mathscr{F}\right\} .
$$

Lema 7.10 Existe $F \in \mathscr{F} \operatorname{com} F(\theta)=0$ e $y=F^{\prime}(\theta)$ se, e somente se, $y \in X_{\theta}$.

Demonstração. O teorema de Riemman (ver Teorema 2.30) garante a existência de uma única aplicação conforme $\varphi: \mathbb{S} \longrightarrow \mathbb{D}$ tal que $\varphi(\theta)=0$ e $\beta=\varphi^{\prime}(\theta)>0$, onde $\mathbb{D}$ é o disco unitário complexo e $\mathbb{S}=\{z: 0<\operatorname{Re}(z)<1\}$

Suponhamos primeiro que existe $F \in \mathscr{F} \operatorname{com} F(\theta)=0$ e $y=F^{\prime}(\theta)$. Definamos $H=\varphi^{-1} F$, isto implica que $H$ pertence a $\mathscr{F},\|H\|_{\mathscr{F}}=\|F\|_{\mathscr{F}}$ e $y=F^{\prime}(\theta)=\beta H(\theta) \in X_{\theta}$.

Recíprocamente suponhamos $y \in X_{\theta}$. Por definição de $X_{\theta}$, temos que existe $G \in \mathscr{F}$ tal que $G(\theta)=x$. Se definimos $F:=\beta^{-1} \varphi G$, obtemos uma função $F$ que está em $\mathscr{F}$, que satisfaz $F(\theta)=0$ e $y=F^{\prime}(\theta)$.

A proposição seguinte fornece a $d X_{\theta}$ de uma norma.

Proposição 7.11 $\|\cdot\|_{d X_{\theta}}$ definida em $d X_{\theta}$ como

$$
\|(x, y)\|_{d X_{\theta}}=\inf \left\{\|F\|_{\mathscr{F}}: x=F(\theta), y=F^{\prime}(\theta)\right\}
$$

é uma norma.

Demonstração. Suponha que $(x, y) \in d X_{\theta} \operatorname{com}\|(x, y)\|_{d X_{\theta}}=0$. Por definição obtemos $\|x\|_{\theta} \leq$ $\|(x, y)\|_{d X_{\theta}}=0$ e assim $x=0$. Existe $F \in \mathscr{F}$ tal que $F(\theta)=0$ e $F^{\prime}(\theta)=y$. Usando as notações 


\subsection{Auto-extensões de espaços interpolados}

do Lema 7.10 obtemos que existe $H \in \mathscr{F}$ tal que $F=\varphi H$ e $y=F^{\prime}(\theta)=\beta H(\theta) \in X_{\theta}$. Agora

$$
\begin{aligned}
\|(0, y)\|_{d X_{\theta}} & =\inf \left\{\|F\|_{\mathscr{F}}: F(\theta)=0, y=F^{\prime}(\theta)\right\} \\
& =\inf \left\{\|\varphi H\|_{\mathscr{F}}: y=\beta H(\theta)\right\} \\
& =\inf \left\{\|H\|_{\mathscr{F}}: y \beta^{-1}=H(\theta)\right\} \\
& =\left\|y \beta^{-1}\right\|_{\theta} \\
& =\beta^{-1}\|y\|_{\theta}
\end{aligned}
$$

portanto

$$
\|(0, y)\|_{d X_{\theta}}=\beta^{-1}\|y\|_{\theta}
$$

Neste caso $y=0$, pois $\|(x, y)\|_{d X_{\theta}}=0$.

É claro que $\|\alpha(x, y)\|_{d X_{\theta}}=|\alpha|\|(x, y)\|_{d X_{\theta}}$ para todo $\alpha$ complexo. Provaremos agora a desigualdade triangular. Sejam $(x, y),(w, z) \in d X_{\theta}$ e $x=F(\theta), y=F^{\prime}(\theta), w=G(\theta), z=G^{\prime}(\theta)$

$$
\begin{aligned}
\|(x, y)+(w, z)\|_{d X_{\theta}} & =\|(x+w, y+z)\|_{d X_{\theta}} \\
& =\inf \left\{\|H\|_{\mathscr{F}}: H(\theta)=x+w, y+z=H^{\prime}(\theta)\right\} \\
& \leq\|F+G\|_{\mathscr{F}} \\
& \leq\|F\|_{\mathscr{F}}+\|G\|_{\mathscr{F}} .
\end{aligned}
$$

Isto implica

$$
\|(x, y)+(w, z)\|_{d X_{\theta}} \leq \inf \left\{\|F\|_{\mathscr{F}}: F(\theta)=x, y=F^{\prime}(\theta)\right\}+\|G\|_{\mathscr{F}},
$$

para qualquer $G$, portanto

$$
\begin{aligned}
\|(x, y)+(w, z)\|_{d X_{\theta}} & \leq\|(x, y)\|_{d X_{\theta}}+\inf \left\{\|G\|_{\mathscr{F}}: G(\theta)=w, y=G^{\prime}(\theta)=z\right\} \\
& \leq\|(x, y)\|_{d X_{\theta}}+\|(w, z)\|_{d X_{\theta}} .
\end{aligned}
$$

Teorema 7.12 O espaço dX $X_{\theta}$ é uma auto-extensão de $X_{\theta}$.

Demonstração. Basta provar que

$$
0 \rightarrow X_{\theta} \stackrel{i}{\rightarrow} d X_{\theta} \stackrel{j}{\rightarrow} X_{\theta} \rightarrow 0
$$

onde $i(x)=(0, x)$ e $j(x, y)=x$, é uma sequência exata curta. O Lema 7.10 garante que $i$ está bem definida. Imediatamente obtemos que $i$ é injetora, $j$ é sobrejetora, $i\left(X_{\theta}\right)=\operatorname{ker}(j)$ e $i, j$ são contínuas, Assim $d X_{\theta}$ é uma auto-extensão de $X_{\theta}$. 
Observação 7.13 Considere a função $\delta_{\theta}: \mathscr{F} \longrightarrow X_{\theta}$ definida por $\delta_{\theta}(F)=F(\theta)$. Note que $\delta_{\theta}$ é limitada e sobrejetora. Então a Proposição 2.54 garante que existe uma função homogênea $B_{\theta}: X_{\theta} \longrightarrow \mathscr{F}$ que verifica $\left\|B_{\theta}(x)\right\|_{\mathscr{F}} \leq C\|x\|_{X_{\theta}}$, para todo $x \in X_{\theta}$ e algum $C>1$, e $\delta_{\theta} \circ B_{\theta}=i d_{X_{\theta}}$. Consideremos a função homogênea $\delta_{\theta}^{\prime}: \mathscr{F} \longrightarrow L_{0}$ definida por $\delta_{\theta}^{\prime}(F)=F^{\prime}(\theta)$ e $\Omega: X_{\theta} \longrightarrow$ $L_{0}$ dada como $\Omega=\delta_{\theta}^{\prime} \circ B_{\theta}$. Note que $\Omega$ assim definida é também homogênea e $\Omega(x)=$ $F^{\prime}(\theta)$, onde $F(\theta)=x$ e $\|F\|_{\mathscr{F}} \leq C\|x\|_{X_{\theta}}$.

Proposição 7.14 Sejam $X_{0}, X_{1}$ espaços de Köthe e $X_{\theta}=X=\left[X_{0}, X_{1}\right]_{\theta}$ e $C>1$ constante. Definimos uma função $\Omega$ em $X$ como

$$
\Omega(x)=F^{\prime}(\theta) \text { onde } F(\theta)=x \text { e }\|F\|_{\mathscr{F}} \leq C\|x\|_{X_{\theta}} .
$$

Então $\Omega$ é um centralizador chamado Centralizador induzido por $\left[X_{0}, X_{1}\right]_{\theta}$.

Demonstração. É imediato provar que $\Omega$ é homogênea. Seja $u \in L_{\infty}$ e $\Omega(x)=F^{\prime}(\theta)$, com $\|F\|_{\mathscr{F}} \leq C\|x\|_{X_{\theta}}$. Então $u F \in \mathscr{F}$ e $\|u F\|_{\mathscr{F}} \leq\|u\|_{\infty}\|F\|_{\mathscr{F}}$. Seja $G \in \mathscr{F}$ com $\Omega(u x)=G^{\prime}(\theta)$ onde $G(\theta)=u x \mathrm{e}\|G\|_{\mathscr{F}} \leq C\|u x\|_{X_{\theta}}$. Observe que

$$
(G-u F)(\theta)=0 \text { e } \Omega(u x)-u \Omega(x)=(G-u F)^{\prime}(\theta) .
$$

Pelo Lema 7.10 (usando a notação do lema 7.10) existem $\varphi: \mathbb{S} \longrightarrow \mathbb{D}, \beta>0$ e $H \in \mathscr{F}$, com $G-u F=\varphi H$ e $(G-u F)^{\prime}(\theta)=\beta H(\theta)$. Agora

$$
\begin{aligned}
\|\Omega(u x)-u \Omega(x)\|_{X_{\theta}} & =\left\|(G-u F)^{\prime}(\theta)\right\|_{X_{\theta}} \\
& =\beta\|H(\theta)\|_{X_{\theta}} \\
& \leq \beta\|H\|_{\mathscr{F}} \\
& =\beta\|G-u F\|_{\mathscr{F}} \\
& \leq \beta\left(\|G\|_{\mathscr{F}}+\|u F\|_{\mathscr{F}}\right) \\
& \leq C \beta\left(\|u x\|_{X_{\theta}}+C\|u\|_{\infty}\|x\|_{\theta}\right) \\
& \leq C(C+1) \beta\|u\|_{\infty}\|x\|_{X_{\theta}} .
\end{aligned}
$$

Observação 7.15 Se nós definimos outra função $\Omega_{1}$ como na Proposição 7.14 , isto é,

$$
\Omega_{1}(x)=F_{1}^{\prime}(\theta) \text { onde } F_{1}(\theta)=x \text { e }\left\|F_{1}\right\|_{\mathscr{F}} \leq C_{1}\|x\|_{X_{\theta}},
$$

então $\Omega_{1}$ e $\Omega$ são limitadamente equivalentes, portanto $d_{\Omega} X_{\theta}$ e $d_{\Omega_{1}} X_{\theta}$ são equivalente. De fato: 
usando as notações do Lema 7.10, temos que $F-F_{1}=\varphi H$, além disso, $\Omega(x)-\Omega_{1}(x)=$ $\left(F-F_{1}\right)^{\prime}(\theta)=\beta H(\theta), \log 0$

$$
\begin{aligned}
\left\|\Omega(x)-\Omega_{1}(x)\right\|_{X_{\theta}} & =\left\|\left(F-F_{1}\right)^{\prime}(\theta)\right\|_{X_{\theta}} \\
& =\beta\|H(\theta)\|_{X_{\theta}} \\
& \leq \beta\|H\|_{\mathscr{F}} \\
& =\beta\|\varphi H\|_{\mathscr{F}} \\
& =\beta\left(\left\|F-F_{1}\right\|_{\mathscr{F}}\right. \\
& \leq C\|x\|_{X_{\theta}}+C_{1}\|x\|_{X_{\theta}} \\
& \leq \beta\left(C+C_{1}\right)\|x\|_{X_{\theta}} .
\end{aligned}
$$

Resumindo, a definição de $d_{\Omega} X_{\theta}$ é independente da escolha de $\Omega$.

Proposição 7.16 Sejam $X_{0}, X_{1}$ espaços de Köthe e $X=\left[X_{0} X_{1}\right]_{\theta}$. Então existem $u_{0} \geq 0 e$ $u_{1} \geq 0$, respectivamente em $X_{0}$ e $X_{1}$ tais que $|x|=u_{0}^{1-\theta} u_{1}^{\theta}$ e $\|x\|_{X_{\theta}}=\left\|u_{0}\right\|_{X_{0}}=\left\|u_{1}\right\|_{X_{1}}$. Uma fatoração deste tipo é chamada de optimal.

Demonstração. Primeiro, assumamos que $\|x\|_{X_{\theta}}=1$. Escolhamos uma sequência $\left(x_{n}\right)_{n}$ em $B_{X_{\theta}}$ convengente em medida para $x$. Na prova da Proposição 5.14 (Passo 4.) encontramos $u_{0} \in B_{X_{0}}$ e $u_{1} \in B_{X_{1}}$, não negativos, tais que $|x|=\left|u_{0}\right|^{1-\theta}\left|u_{1}\right|^{\theta}$. Como $\|x\|_{X_{\theta}}=1$, temos que $\left\|u_{0}\right\|_{X_{0}}=\left\|u_{1}\right\|_{X_{1}}=1$. Agora assuma que $\|x\|_{X_{\theta}} \neq 0$. Então existem $w_{0} \in X_{0}$ e $w_{1} \in X_{1}$ unitários não negativos tais que

$$
\frac{|x|}{\|x\|_{X_{\theta}}}=\left|w_{0}\right|^{1-\theta}\left|w_{1}\right|^{\theta},
$$

isto é, $|x|=\left(\|x\|_{\theta}\left|w_{0}\right|\right)^{1-\theta}\left(\left|w_{1}\right|\|x\|_{\theta}\right)^{\theta}$. Se $u_{0}=\|x\|_{\theta} \cdot w_{0}$ e $u_{0}=\|x\|_{\theta} \cdot w_{1}$, então $u_{0}$ e $u_{1}$ são funções não negativas em $X_{0}$ e $X_{1}$, respectivamente, que verificam $|x|=u_{0}^{1-\theta} u_{1}^{\theta}$ e $\|x\|_{X_{\theta}}=\left\|u_{0}\right\|_{X_{0}}=\left\|u_{1}\right\|_{X_{1}}$.

Observação 7.17 (Fórmula explícita de centralizador induzido) A Observação 7.15 é útil para encontrar uma fórmula explícita de $\Omega$ (centralizador induzido por $\left[X_{0} X_{1}\right]$ ). Se $x \in X_{\theta} e$ $|x|=u_{0}^{1-\theta} u_{1}^{\theta}$, onde $u_{0} \in X_{0}$ e $u_{1} \in X_{1}$, é uma fatoração optimal para $x$, então

$$
\Omega(x)=x\left(\log u_{1}-\log u_{0}\right) .
$$

De fato:

escrevamos $x(s)=|x(s)| \cdot \zeta(s)$, onde $\zeta$ é uma função de valor complexo tal que $|\zeta(s)|=1$ para todo $s \in S$. Seja $F(z)=u_{0}^{1-z} u_{1}^{z} \cdot \zeta$. Verifiquemos que $F \in \mathscr{F}$. De fato, é imediato ver que 
$\left|u_{0}^{1-i t} u_{1}^{i t} \cdot \zeta\right|=u_{0}$, daí resulta que $F(i t) \in X_{0}$. De maneira análoga obtemos $\left|u_{0}^{1-(1+i t)} u_{1}^{1+i t} \cdot \zeta\right|=u_{1}$. Portanto, $F(1+i t) \in X_{1}$. Observe que

$$
\begin{aligned}
\|F\|_{\mathscr{F}} & =\max \left(\sup _{t}\|F(i t)\|_{X_{0}}, \sup _{t}\|F(1+i t)\|_{X_{1}}\right) \\
& =\max \left(\sup _{t}\left\|\left(u_{0}\right)^{1-i t}\left(u_{1}\right)^{i t} \cdot \zeta\right\|_{X_{0}}, \sup _{t}\left\|\left(u_{0}\right)^{i t}\left(u_{1}\right)^{1+i t} \cdot \zeta\right\|_{X_{1}}\right) \\
& =\max \left(\sup _{t}\left\|\left|\left(u_{0}\right)^{1-i t}\left(u_{1}\right)^{i t}\right|\right\|_{X_{0}}, \sup _{t}\left\|\left|\left(u_{0}\right)^{i t}\left(u_{1}\right)^{1+i t}\right|\right\|_{X_{1}}\right) \\
& =\max \left(\left\|u_{0}\right\|_{X_{0}},\left\|u_{1}\right\|_{X_{1}}\right) \\
& =\left\|x_{\theta}\right\|_{X_{\theta}}
\end{aligned}
$$

Os cálculos acima provam que $\|F\|_{\mathscr{F}}<\infty$. Observe que $F$ possui derivada $F^{\prime}(z)=F(z)\left(\log u_{1}-\right.$ $\log u_{0}$ ) e assim

$$
\Omega(x)=x\left(\log u_{1}-\log u_{0}\right)
$$

O teorema seguinte mostra que é possível identicar $d_{\Omega} X_{\theta}$ com $d X_{\theta}$, o que significa que $d_{\Omega} X_{\theta}$ pode ser fornecido de uma norma equivalente a $\|\cdot\|_{\Omega_{\Omega} X_{\theta}}$.

Teorema 7.18 Seja $\Omega$ o centralizador induzido em $X_{\theta}=\left[X_{0} X_{1}\right]_{\theta}$. O diagrama seguinte



é comutativo, onde $i, j$ e $i^{\prime}, j^{\prime}$ são definidos como no Teorema 7.12 e a Observação 3.10, respectivamente, e $T(x, y)=(x, y)$.

Demonstração. Primeiro mostraremos que $T$ está bem definido. Para provar que o diagrama comuta é suficiente mostrar que $T$ é limitado ( ver Proposição 2.69).

Suponha $(x, y) \in d_{\Omega} X_{\theta}$, isto é, $x \in X_{\theta}$ e $y-\Omega(x) \in X_{\theta}$. Seja $\Omega(x)=F^{\prime}(\theta) \operatorname{com} F(\theta)=x$ e $\|F\|_{\mathscr{F}} \leq C\|x\|_{X_{\theta}}$, onde $C>1$. Existe $G \in \mathscr{F}$ tal que $G(\theta)=y-\Omega(x)$ e $\|G\|_{\mathscr{F}} \leq C\|y-\Omega(x)\|_{X_{\theta}}$. Seja $\varphi$ e $\beta>0$ como no Lema 7.10, definimos $J=\beta^{-1} \varphi G$ em $\mathscr{F}$. Observe que $J(\theta)=$ $0, J^{\prime}(\theta)=y-\Omega(x)$ e $\|J\|_{\mathscr{F}} \leq \beta^{-1} C\|y-\Omega(x)\|_{X_{\theta}}$ para algum $K>0$. Agora $y=(y-\Omega(x))+\Omega(x)=$ $(J+F)^{\prime}(\theta)$ e também $(J+F)(\theta)=x$, onde $J+F \in \mathscr{F}$, e assim provamos que $(x, y) \in d X_{\theta}$. Isto prova que $T$ está bem definido. Afirmamos que $T$ é limitado. De fato: 


$$
\begin{aligned}
\|(x, y)\|_{d X_{\theta}} & =\inf \left\{\|H\|_{\mathscr{F}}: H(\theta)=x, H^{\prime}(\theta)=y\right\} \\
& \leq\|F+J\|_{\mathscr{F}} \\
& \leq\|F\|_{\mathscr{F}}+\|J\|_{\mathscr{F}} \\
& \leq C\|x\|_{X_{\theta}}+C \beta^{-1}\|y-\Omega(x)\|_{X_{\theta}} \\
& \leq \max \left(C, C \beta^{-1}\right)\left(\|x\|_{X_{\theta}}+\|y-\Omega(x)\|_{X_{\theta}}\right) \\
& =\max \left(C, C \beta^{-1}\right)\|(x, y)\|_{d_{\Omega} X_{\theta}} .
\end{aligned}
$$

isto mostra que $d X_{\theta}=d_{\Omega} X_{\theta}$ (a igualdade é um isomorfismo topologico).

Proposição 7.19 Sejam $X_{0}$ e $X_{1}$ espaço de Köthe e $X=\left[X_{0} X_{1}\right]_{\theta}$. Seja $\Omega$ o centralizador induzido por $X_{0}, X_{1}$ e $\theta$. Se $\Omega^{1}$ é o centralizador em $L_{1}$ definido na Proposição 7.6, então para $f \in L_{1}$, temos que

$$
\Omega^{1}(f)=f\left(\log x_{1}-\log x_{0}\right),
$$

onde $|f|=x_{0} x_{0}^{*}=x_{1} x_{1}^{*}$ são as fatorações de Losanovskii de $f$ respecto $X_{0}$ e $X_{1}$.

Demonstração. Assumamos que $\|f\|_{L_{1}}=1 \mathrm{e}|f|=x x^{*}$ é a fatoração de Losanovskii de $f$ em relação a $X=\left[X_{0} X_{1}\right]_{\theta}$, isto é $\|x\|_{X}=\left\|x^{*}\right\|_{X^{*}}=1, \operatorname{supp}(f)=\operatorname{supp}(x)=\operatorname{supp}\left(x^{*}\right), x \geq 0$.

Em $\mathbb{S}=\{z: 0<\mathfrak{R e}(z)<1\}$ definamos duas funções como $G(z)=\left(x_{0}\right)^{1-z}\left(x_{1}\right)^{z}$ e $H(z)=$ $\left(x_{0}^{*}\right)^{1-z}\left(x_{1}^{*}\right)^{z}$. Vejamos que $G(\theta) H(\theta)$ é a fatoração de Lozanovskii de $f$. De fato, observe que

$$
\|G(\theta)\|_{X}=\left\|x_{0}^{1-\theta} x_{1}^{\theta}\right\|_{X} \leq\left\|x_{0}\right\|_{X_{0}}^{1-\theta}\left\|x_{1}\right\|_{X_{1}}^{\theta} \leq 1
$$

$\mathrm{e}$

$$
\|H(\theta)\|_{X^{*}}=\left\|\left(x_{0}^{*}\right)^{1-\theta}\left(x_{1}^{*}\right)^{\theta}\right\|_{X^{*}} \leq\left\|x_{0}^{*}\right\|_{X_{0}^{*}}^{1-\theta}\left\|x_{1}^{*}\right\|_{X_{1}^{*}}^{\theta} \leq 1,
$$

pois lembre-se que $\left(X_{0}^{*}\right)^{1-\theta}\left(X_{1}^{*}\right)^{\theta} \subset X^{*}=\left(X_{0}^{1-\theta} X_{1}^{\theta}\right)^{*}$ (ver Proposição 5.12). Note que $G(\theta) H(\theta)=$ $|f| \mathrm{e}$

$$
1=\|f\|_{L_{1}}=\|G(\theta) H(\theta)\|_{L_{1}} \leq\|G(\theta)\|_{X}\|H(\theta)\|_{X^{*}} \leq 1,
$$

$\operatorname{logo}\|G(\theta)\|_{X}=\|H(\theta)\|_{X^{*}}=1$. Note também que

$$
\operatorname{supp}(G(\theta))=\operatorname{supp}\left(x_{0}\right) \cap \operatorname{supp}\left(x_{1}\right)=\operatorname{supp}(f) .
$$

Analogamente vemos que $\operatorname{supp}(H(\theta))=\operatorname{supp}(f)$. Como $G(\theta) \geq 0$, concluimos que $G(\theta) H(\theta)=$ $|f|$ é a fatoração de Lozanovskii de $f$, portanto, $x_{0}^{1-\theta} x_{1}^{\theta}=G(\theta)=x$ e $H(\theta)=x^{*}$ pela unicidade da fatoração de Lozanovskii. Agora vamos demonstrar que a única fatoração optimal de $|x|$ é $|x|=x_{0}^{1-\theta} x_{1}^{\theta}$. De fato, seja $x=u_{0}^{1-\theta} u_{1}^{\theta}$ uma fatoração optimal para $x$, isto é, $\left\|u_{0}\right\|_{X_{0}}=\left\|u_{1}\right\|_{X_{1}}=1$. Definamos outra função $K$ em $\mathbb{S}$ por $K(z)=u_{0}^{1-z} u_{1}^{z}$. Observe que

$$
\begin{aligned}
|K(i t) H(i t)| & =\left|u_{0}^{1-i t} u_{1}^{i t}\left(x_{0}^{*}\right)^{1-i t}\left(x_{1}^{*}\right)^{i t}\right| \\
& =\left|u_{0}\right|\left|x_{0}^{*}\right|,
\end{aligned}
$$


$\log 0$

$$
\int|K(i t) H(i t)| d \mu=\int\left|u_{0}\left\|x_{0}^{*} \mid d \mu \leq\right\| x_{0}^{*} \|_{X^{*}}=1 .\right.
$$

Analogamente mostramos que

$$
|K(1+i t) H(1+i t)|=\left|u_{1}\right|\left|x_{1}^{*}\right|
$$

Portanto $\int|K(1+i t) H(1+i t)| d \mu \leq 1$. Pelo Principio do Módulo Máximo, como $\int|G(\theta) H(\theta)| d \mu=$ 1 , onde $\theta$ pertence ao interior de $\overline{\mathbb{S}}$, temos que

$$
\int|K(z) H(z)| d \mu=1
$$

para qualquer $z \in \mathbb{S}$. Agora $\left|\int K(z) H(z) d \mu\right| \leq \int|K(z) H(z)| d \mu=1$ para qualquer $z \in \mathbb{S}$, além de $\int K(\theta) H(\theta) d \mu=1$ temos $\int K(z) H(z) d \mu=1$ para qualquer $z \in \mathbb{S}$. Nós provamos que

$$
\int K(z) H(z) d \mu=\int|G(z) H(z)| d \mu,
$$

para $z \in \mathbb{S}$, consequentemente

$$
\int \operatorname{Re}(k(z) H(z)) d \mu+i \int \operatorname{Im}(K(z) H(z)) d \mu=\int|K(z) H(z)| d \mu .
$$

Desta igualde obtemos que $K(z) H(z)$ é sempre real não negativo. Por outro lado

$$
\begin{aligned}
K(\theta+i t) H(\theta+i t) & =|K(\theta+i t) H(\theta+i t)| \\
& =\left|u_{0}^{1-\theta-i t} u_{1}^{\theta+i t}\left(x_{0}^{*}\right)^{1-\theta-i t}\left(x_{1}^{*}\right)^{\theta+i t}\right| \\
& =\left|u_{0}^{1-\theta} u_{1}^{\theta}\left(x_{0}^{*}\right)^{1-\theta}\left(x_{1}^{*}\right)^{\theta}\right| \\
& =|x H(\theta)| \\
& =|x|\left|x^{*}\right| \\
& =|f|
\end{aligned}
$$

para todo $t \in \mathbb{R}$. Lembre-se que as funções $K$ e $H$ definidas acimas dependem também de $s \in S$ (espaço polonês). Para cada $s \in S$ definamos $g_{s}: \mathbb{S} \rightarrow \mathbb{R}$ como $g_{s}(z)=K(z) H(z)-|f|$. A função $g_{s}$ é holomorfa em $\mathbb{S}$ e qualquer elemento da forma $\theta+i t$ é um zero de $g_{s}$, portanto existe uma sequência de pontos distintos em $\mathbb{S}$ com ponto de acumulação no interior de $\mathbb{S}$, tais que anulam a $g_{s}$. Isto mostra que $g_{s}$ é nula em $\mathbb{S}$, e assim $K(z) H(z)=|f|$ para todo $z \in \mathbb{S}$, isto é, $u_{0}^{1-z} u_{1}^{z}\left(x_{0}^{*}\right)^{1-z}\left(x_{1}^{*}\right)^{z}=|f|$ para todo $z \in \mathbb{S}$, em particular, para $z=0$ e $z=1$ (estendendo continuamente à fronteira de $\mathbb{S}$ ), respectivamente, obtemos $u_{0} x_{0}^{*}=|f|$ e $u_{1} x_{1}^{*}=|f|$, como $u_{i}, x_{i}^{*} \geq 0$ para $i=0,1$, isto é , $u_{0} x_{0}^{*}=x_{0} x_{0}^{*}$ e $u_{1} x_{1}^{*}=x_{1} x_{1}^{*}$, assim $u_{i}(s)=x_{i}(s)$ para $i=0,1$, se 
$s \in \operatorname{supp}(f)$. A Observação 7.17, permite escrever $\Omega(x)=x\left(\log u_{1}-\log u_{0}\right)=x\left(\log x_{1}-\log x_{0}\right)$. Na Proposição 7.6 definimos $\Omega^{1}$ pondo $\Omega^{1}(f)=x^{*} \Omega(x)$, portanto $\Omega^{1}(f)=f\left(\log x_{1}-\log x_{0}\right)$ sempre que $\|f\|_{L_{1}}=1$. Quando $\|f\|_{L_{1}}$ é diferente de 1 e positivo, o resultado é imediato pelo fato de $\Omega^{1}$ ser homogêneo.

Definição 7.20 Seja $\Omega$ um centralizador em X. Definamos um conjunto e uma função, respectivamente, como

$$
\mathscr{T}_{\Omega}=\left\{f \in L_{1}^{+}(\mu): \Omega^{1}(f) \in L_{1}(\mu)\right\}
$$

$e \Phi^{\Omega}: \mathscr{T}_{\Omega} \longrightarrow \mathbb{R}$ por

$$
\Phi^{\Omega}(f)=\int \Omega^{1}(f) d \mu
$$

onde $\Omega^{1}$ é o Centralizador em $L_{1}$ definido na Proposicão 7.19.

A proposição seguinte prova que $\mathscr{T}_{\Omega}$ é um ideal e que $\Phi^{\Omega}$ é semilinear.

Proposição 7.21 $\mathscr{T}_{\Omega}$ e $\Phi^{\Omega}$ definidos acima são, respectivamente, um ideal e uma aplicação semilinear.

Demonstração. Vejamos primeiro que $\mathscr{T}_{\Omega}$ é um semi-ideal de $L_{1}^{+}$. De fato, se $f, g \in \mathscr{T}_{\Omega}$, então

$$
\left|\Omega^{1}(f+g)\right| \leq\left|\Omega^{1}(f+g)-\Omega^{1}(f)-\Omega^{1}(g)\right|+\left|\Omega^{1}(f)\right|+\left|\Omega^{1}(g)\right| .
$$

Como $\Omega^{1}(f+g)-\Omega^{1}(f)-\Omega^{1}(g) \in L_{1}\left(\right.$ ver Proposição 7.2) e $\Omega^{1}(f), \Omega^{1}(g)$ pertence a $L_{1}$ obtemos que $\Omega^{1}(f+g) \in L_{1}$, que equivale a dizer $f+g \in \mathscr{T}_{\Omega}$.

Assuma que $0 \leq f \leq g$, com $g \in \mathscr{T}_{\Omega}$, isto é $\Omega_{1}(g) \in L_{1}$. Note que $\frac{f}{g} \in L_{\infty}$. Pelo fato de ser $\Omega^{1}$ um centralizador, temos que $\Omega^{1}(f)-\frac{f}{g} \Omega^{1}(g) \in L_{1}$, assim

$$
\Omega^{1}(f)=\left(\Omega^{1}(f)-\frac{f}{g} \Omega^{1}(g)\right)+\frac{f}{g} \Omega^{1}(g) \in L_{1} .
$$

Demonstraremos que $\Phi^{\Omega}$ é um funcional semilinear sobre $\mathscr{T}_{\Omega}$. De fato, pelo Corolário 7.9 deduzimos que

$$
\left\|\Omega^{1}(f+g)-\Omega^{1}(f)-\Omega^{1}(g)\right\|_{L_{1}} \leq 15 \rho(\Omega)\left(\|f\|_{L_{1}}+\|g\|_{L_{1}}\right),
$$

portanto,

$$
\left|\Phi^{\Omega}(f+g)-\Phi^{\Omega}(f)-\Phi^{\Omega}(g)\right| \leq 15 \rho(\Omega)\left(\|f\|_{L_{1}}+\|g\|_{L_{1}}\right) .
$$

O funcional $\Phi^{\Omega}$ é homogêneo devido a que $\Omega^{1}$ é homogêneo. 
Seja $0 \leq f_{n} \leq f$, tal que $f \in \mathscr{T}_{\Omega}$ e $\lim _{n \rightarrow \infty}\left\|f_{n}\right\|_{L_{1}}=0$. Demonstraremos que $\lim _{n \rightarrow \infty} \Phi^{\Omega}\left(f_{n}\right)=0$. De fato, como $\lim _{n \rightarrow \infty}\left\|f_{n}\right\|_{L_{1}}=0$, então existe uma subsequência de $\left(f_{n}\right)$, que denotaremos por $\left(f^{(1)}\right)_{n}$, tal que $f_{n}^{(1)} \stackrel{n \rightarrow \infty}{\rightarrow} 0$ q.s. Definamos $g_{m}=\sup _{k \geq m} f_{k}^{(1)}$. Note que $g_{m} \in \mathscr{T}_{\Omega}, 0 \leq f_{n}^{(1)} \leq g_{m} \leq f$ se $n \geq m$ e $g_{m} \rightarrow 0$ q.s., se $m \rightarrow \infty$. Portanto, pelo Teorema da Convergência Dominada, temos que $\lim _{m \rightarrow \infty}\left\|g_{m}\right\|_{L_{1}}=0$. Devido a que $\Omega^{1}$ é um centralizador em $L_{1}$ e $0 \leq f_{n}^{(1)} \cdot g_{m}^{-1} \leq 1$, se $n \geq m$, deduzimos que

$$
\left\|\Omega^{1}\left(f_{n}^{(1)}\right)-f_{n}^{(1)} g_{m}^{-1} \Omega^{1}\left(g_{m}\right)\right\|_{L_{1}} \leq 5 \rho(\Omega)\left\|g_{m}\right\|_{L_{1}}, \text { se } n \geq m .
$$

Como $\Omega\left(g_{m}\right) \in L_{1}$,

$$
\left|f_{n}^{(1)} g_{m}^{-1} \Omega^{1}\left(g_{m}\right)\right| \leq\left|\Omega^{1}\left(g_{m}\right)\right|,
$$

$\mathrm{e}$

$$
\left|f_{n}^{(1)} g_{m}^{-1} \Omega^{1}\left(g_{m}\right)\right| \rightarrow 0 \text { q.s. }
$$

obtemos pelo Teorema da Convergência Dominada que

$$
\lim _{n \rightarrow \infty}\left\|f_{n}^{(1)} g_{m}^{-1} \Omega^{1}\left(g_{m}\right)\right\|_{L_{1}}=0,
$$

para $m \in \mathbb{N}$ fixo.

Consequentemente, existe uma subsequência $\left(f_{n}^{(1)}\right)_{n}$ de $\left(f_{n}\right)_{n}$ tal que

$$
\begin{aligned}
\left\|\Omega^{1}\left(f_{n}^{(1)}\right)\right\|_{L_{1}} & \leq\left\|\Omega^{1}\left(f_{n}^{(1)}\right)-f_{n}^{(1)} g_{m}^{-1} \Omega^{1}\left(g_{m}\right)\right\|_{L_{1}}+\left\|f_{n}^{(1)} g_{m}^{-1} \Omega^{1}\left(g_{m}\right)\right\|_{L_{1}} \\
& \leq 5 \rho(\Omega)\left\|g_{m}\right\|_{L_{1}}+\left\|f_{n}^{(1)} g_{m}^{-1} \Omega^{1}\left(g_{m}\right)\right\|_{L_{1}},
\end{aligned}
$$

se $n \geq m$. Logo,

$$
\underset{n \rightarrow \infty}{\limsup }\left\|\Omega^{1}\left(f_{n}^{(1)}\right)\right\|_{L_{1}} \leq 5 \rho(\Omega)\left\|g_{m}\right\|_{L_{1}} .
$$

Além disso, se $m \rightarrow \infty$, então

$$
\underset{n \rightarrow \infty}{\limsup }\left\|\Omega^{1}\left(f_{n}^{(1)}\right)\right\|_{L_{1}}=0 .
$$

Portanto

$$
\lim _{n \rightarrow \infty}\left\|\Omega^{1}\left(f_{n}^{(1)}\right)\right\|_{L_{1}}=0 .
$$

Até o momento demonstramos que para toda sequência $\left(f_{n}\right)_{n}$ tal que $0 \leq f_{n} \leq f$ para todo $n \in \mathbb{N}$ e $\lim _{n \rightarrow \infty}\left\|f_{n}\right\|_{L_{1}}=0$, existe uma subsequência $\left(f_{n}^{(1)}\right)_{n}$ de $\left(f_{n}\right)_{n}$ tal que

$$
\lim _{n \rightarrow \infty}\left\|\Omega^{1}\left(f_{n}^{(1)}\right)\right\|_{L_{1}}=0 .
$$

Agora demonstraremos que se $\left(f_{n}\right)_{n}$ é uma sequência tal que $0 \leq f_{n} \leq f$ para todo $n \in \mathbb{N}$ e $\lim _{n \rightarrow \infty}\left\|f_{n}\right\|_{L_{1}}=0$, então

$$
\lim _{n \rightarrow \infty}\left\|\Omega^{1}\left(f_{n}\right)\right\|_{L_{1}}=0
$$


De fato, assumamos por contradição que existe uma subequência de $\left(f_{n}\right)_{n}$, que denotaremos por $\left(f_{n}^{(1)}\right)_{n}$, tal que

$$
\left\|\Omega^{1}\left(f_{n}^{(1)}\right)\right\|_{L_{1}}>\varepsilon, \quad \forall n \in \mathbb{N}
$$

e para algum $\varepsilon>0$. Note que $\left\|f_{n}^{(1)}\right\|_{L_{1}} \rightarrow 0$, se $n \rightarrow 0$. Portanto, exite uma subsequência de $\left(f_{n}^{(1)}\right)_{n}$, que denotaremos por $\left(f_{n}^{(2)}\right)_{n}$, tal que

$$
\lim _{n \rightarrow \infty}\left\|\Omega^{1}\left(f_{n}^{(2)}\right)\right\|_{L_{1}}=0 .
$$

Isto contraria o fato (7.10). Consequentemente

$$
\lim _{n \rightarrow \infty}\left\|\Omega^{1}\left(f_{n}\right)\right\|_{L_{1}}=0
$$

$\operatorname{logo} \lim _{n \rightarrow \infty} \Phi^{\Omega}\left(f_{n}\right)=0$ e segue que $\Phi^{\Omega}$ é uma aplicação semilinear.

Proposição 7.22 Sejam $X_{1}, X_{0}$ espaços de Köthe e $X=\left[X_{0} X_{1}\right]_{\theta}$. Seja $\Phi^{\Omega}$ o funcional semilinear definido anteriormente. Então para $f \in \mathscr{T}_{X_{0}} \cap \mathscr{T}_{X_{1}} \cap \mathscr{T}_{\Omega}$ temos que

$$
\Phi^{\Omega}=\Phi_{X_{1}}-\Phi_{X_{0}}
$$

Demonstração. Na prova da Proposição 6.8 provamos que se $f=x y$ é a fatoração de Losanovskii, onde $x \in X$ e $y \in X^{*}$, então

$$
\int f \log |x| d \mu=\sup _{\|z\|_{X} \leq 1} \int f \log |z| d \mu .
$$

Na Proposição 7.19 demonstramos que $\Omega^{1}(f)=f\left(\log x_{1}-\log x_{0}\right)$, onde $|f|=x_{0} x_{0}^{*}$ e $|f|=x_{1} x_{1}^{*}$ são as fatorações de Lozanovskii de $|f| \operatorname{em} X_{0}$ e $X_{1}$, respectivamente. Integrando $\Omega^{1}(f)$, obtemos que

$$
\Phi^{\Omega}=\Phi_{X_{1}}-\Phi_{X_{0}} .
$$




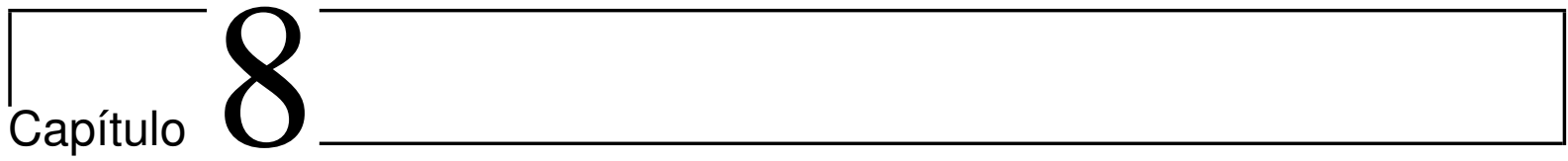

\section{Teoremas de extrapolação}

Antes de começar as provas dos teoremas, vamos fazer uma observação

Observação 8.1 Seja X um espaço de Köthe p-convexo e q-côncavo, onde $\frac{1}{p}+\frac{1}{q}=1$ e $1<$ $p \leq 2$. Destas desigualdades deduzimos imediatamente que $q \geq 2$. A Proposição 2.39 garante que $X^{\prime}$ é um espaço p-convexo e q-côncavo. Por $X^{*}$ ser isométrico a um subespaço de $X^{\prime}$, temos que $X^{*}$ é p-convexo e q-côncavo (ver Proposição 2.40).

Teorema 8.2 (Pisier) [16, pág.498] Se X é um espaço de Köthe p-convexo e q-côncavo, onde $\frac{1}{p}+\frac{1}{q}=1$, então para $\frac{\theta}{2}=\frac{1}{q}$ e $0<\theta<1$ existe um único espaço de Köthe $Y$ tal que

$$
X=L_{2}^{\theta} Y^{1-\theta}
$$

Demonstração. Note que as desigualdades $1 / p+1 / q=1, \theta / 2=1 / q$ e $0<\theta<1$, implicam que $1<p \leq 2$.

Como $X$ é um espaço de Köthe $p$-convexo, então $X^{p}$ é um espaço de Köthe (ver Proposição 5.15 ). O Teorema 6.15 garante que $\Phi_{X^{p}}$ e $\Lambda-\Phi_{X^{p}}$ são funções convexas e pela Proposição 6.10 obtemos que $\Phi_{X^{p}}=p \Phi_{X}$. Portanto $\Lambda-p \Phi_{X}$ é uma função convexa, assim $\frac{1}{p} \Lambda-\Phi_{X}$ é convexa. Por $X$ ser um espaço $q$-côncavo , temos que $X^{*}$ é um espaço $p$-convexo, $\log \frac{1}{p} \Lambda-\Phi_{X^{*}}$ é uma função convexa.

Seja agora

$$
\Phi=\frac{1}{1-\theta} \Phi_{X}-\frac{\theta}{2(1-\theta)} \Lambda
$$

A função $\Phi$ é homogênea, pois $\Phi_{X}$ e $\Lambda$ são homogêneas. Vejamos que $\Phi$, assim definida é uma função convexa. De fato, pelo fato de que $\frac{1}{p} \Lambda-\Phi_{X^{*}}$ é uma função convexa, temos que 


$$
\begin{aligned}
\Delta_{\frac{1}{p} \Lambda-\Phi_{X^{*}}}(f, g) \geq 0 & \Leftrightarrow \frac{1}{p} \Delta_{\Lambda}(f, g)-\Delta_{\Phi_{X^{*}}}(f, g) \geq 0 \\
& \Leftrightarrow \frac{1}{p} \Delta_{\Lambda}(f, g)-\Delta_{\Lambda}(f, g)+\Delta_{\Phi_{X}}(f, g) \geq 0 \\
& \Leftrightarrow \Delta_{\Phi_{X}}(f, g)-\frac{1}{q} \Delta_{\Lambda}(f, g) \geq 0 \\
& \Leftrightarrow \Delta_{\Phi_{X}}(f, g)-\frac{\theta}{2} \Delta_{\Lambda}(f, g) \geq 0 \\
& \Leftrightarrow \frac{1}{1-\theta} \Delta_{\Phi_{X}}(f, g)-\frac{\theta}{2(1-\theta)} \Delta_{\Lambda}(f, g) \geq 0 \\
& \Leftrightarrow \Delta_{\Phi}(f, g) \geq 0 .
\end{aligned}
$$

Demonstraremos similarmente que $\Lambda-\Phi$ é uma função convexa. De fato, similarmente, pelo fato de que $\frac{1}{p} \Lambda-\Phi_{X}$ é uma função convexa, temos que

$$
\begin{aligned}
\Delta_{\frac{1}{p} \Lambda-\Phi_{X}}(f, g) \geq 0 & \Leftrightarrow\left(1-\frac{1}{q}\right) \Delta_{\Lambda}(f, g)-\Delta_{\Phi_{X}}(f, g) \geq 0 \\
& \Leftrightarrow\left(1-\frac{\theta}{2}\right) \Delta_{\Lambda}(f, g)-\Delta_{\Phi_{X}}(f, g) \geq 0 \\
& \Leftrightarrow \frac{2-\theta}{2} \Delta_{\Lambda}(f, g)-\Delta_{\Phi_{X}}(f, g) \geq 0 \\
& \Leftrightarrow \frac{2-\theta}{2(1-\theta)} \Delta_{\Lambda}(f, g)-\frac{1}{1-\theta} \Delta_{\Phi_{X}}(f, g) \geq 0 \\
& \Leftrightarrow \Delta_{\Lambda}(f, g)-\frac{1}{1-\theta} \Delta_{\Phi_{X}}(f, g)+\frac{\theta}{2(1-\theta)} \Delta_{\Lambda}(f, g) \geq 0 \\
& \Leftrightarrow \Delta_{\Lambda}(f, g)-\Delta_{\Phi}(f, g) \geq 0 \\
& \Leftrightarrow \Delta_{\Lambda-\Phi}(f, g) \geq 0 .
\end{aligned}
$$

Portanto, existe um espaço de Köthe $Y$ tal que $\Phi_{Y}=\Phi$ (ver Teorema 6.20). Isto é, 


$$
\begin{aligned}
\Phi_{Y}=\frac{1}{1-\theta} \Phi_{X}-\frac{\theta}{2(1-\theta)} \Lambda & \Leftrightarrow \Phi_{X}=\frac{\theta}{2} \Lambda+(1-\theta) \Phi_{Y} \\
& \Leftrightarrow \Phi_{X}=\Phi_{L_{2}^{\theta} Y^{1-\theta}},
\end{aligned}
$$

e pela unicidade do Teorema 6.20 obtemos $X=L_{2}^{\theta} Y^{1-\theta}$.

Se $Y_{0}$ é um espaço de Köthe que satisfaz $X=L_{2}^{\theta} Y_{0}^{1-\theta}$, então

$$
\Phi_{Y_{0}}=\frac{1}{1-\theta} \Phi_{X}-\frac{\theta}{2(1-\theta)} \Lambda=\Phi_{Y}
$$

Logo $Y_{0}=Y_{1}$, o que prova a unicidade do teorema.

Teorema 8.3 Seja $X$ um espaço de Köthe e $0<\theta<1$. Suponha que existem espaços de Köthe $X_{0}, X_{1}, Y_{0}$ e $Y_{1}$ tais que $X=\left[X_{0}, X_{1}\right]_{\theta}$ e $X=\left[Y_{0}, Y_{1}\right]_{\theta}$. Se $\Omega$ e $\Omega_{0}$ são os centralizadores induzidos por $\left[X_{0}, X_{1}\right]_{\theta}$ e $\left[Y_{0}, Y_{1}\right]_{\theta}$, respectivamente, e são limitadamente equivalentes, então $X_{0}=Y_{0}$ e $X_{1}=Y_{1}$ (os espaços são iguais como normas equivalentes).

Demonstração. Pela Proposição 7.22 temos que

$$
\Phi^{\Omega}=\Phi_{X_{1}}-\Phi_{X_{0}}
$$

$\mathrm{e}$

$$
\Phi^{\Omega_{0}}=\Phi_{Y_{1}}-\Phi_{Y_{0}} .
$$

Pela Proposição 6.9, obtemos que

$$
\Phi_{X}=(1-\theta) \Phi_{X_{0}}+\theta \Phi_{X_{1}}
$$

e

$$
\Phi_{X}=(1-\theta) \Phi_{Y_{0}}+\theta \Phi_{Y_{1}} .
$$

Agora

$$
\begin{aligned}
(1-\theta)\left(\Phi^{\Omega}-\Phi^{\Omega_{0}}\right)= & (1-\theta) \Phi^{\Omega}-(1-\theta) \Phi^{\Omega_{0}}+\Phi_{X}-\Phi_{X} . \\
& \text { Substituindo as equações acimas, temos } \\
= & (1-\theta)\left(\Phi_{X_{1}}-\Phi_{X_{0}}\right)-(1-\theta)\left(\Phi_{Y_{1}}-\Phi_{Y_{0}}\right)+\left(\theta \Phi_{X_{1}}+(1-\theta) \Phi_{X_{0}}\right) \\
& -\left(\theta \Phi_{Y_{1}}+(1-\theta) \Phi_{Y_{0}}\right) \\
= & \Phi_{X_{1}}-\Phi_{Y_{1}} .
\end{aligned}
$$


Como $\Omega$ e $\Omega_{0}$ são limitadamente equivalentes, então $\Omega^{1}$ e $\Omega_{0}^{1}$ são limitadamente equivalentes (ver Corolário 7.8), portanto

$$
\begin{aligned}
\left|\Phi^{\Omega}(f)-\Phi^{\Omega_{0}}(f)\right| & =\left|\int \Omega^{1}(f) d \mu-\int \Omega_{0}^{1}(f) d \mu\right| \\
& \leq\left\|\Omega^{1}(f)-\Omega_{0}^{1}(f)\right\|_{L_{1}} \\
& \leq C_{1}\|f\|_{L_{1}}
\end{aligned}
$$

para todo $f \in \mathscr{T}_{\Omega} \cap \mathscr{T}_{\Omega_{0}}$ e para alguma constante $C_{1}>0$.

Em particular, se $f \in \mathscr{T}$, com $\|f\|_{L_{1}} \leq 1$, onde $\mathscr{T}$ é um semi-ideal estrito contido em $\mathscr{T}_{X_{0}} \cap$ $\mathscr{T}_{X_{1}} \cap \mathscr{T}_{Y_{0}} \cap \mathscr{T}_{Y_{1}} \cap \mathscr{T}_{\Omega} \cap \mathscr{T}_{\Omega_{0}}$, temos que

$$
\begin{aligned}
d\left(\Phi_{X_{1}}, \Phi_{Y_{1}}\right) & =\sup _{\|f\|_{L_{1}} \leq 1}\left|\Phi_{Y_{1}}(f)-\Phi_{X_{1}}(f)\right| \\
& =(1-\theta) \cdot \sup _{\|f\|_{L_{1}} \leq 1}\left|\Phi^{\Omega}(f)-\Phi^{\Omega_{0}}(f)\right| \\
& \leq(1-\theta) C_{1},
\end{aligned}
$$

portanto o Corolário 6.18 garante que $X_{1}$ e $Y_{1}$ são iguais com normas equivalentes. Analogamente se prova que $X_{0}$ e $Y_{0}$ são iguais com normas equivalentes.

Teorema 8.4 Sejam X um espaço de Köthe p-convexo e q-côncavo, onde $\frac{1}{p}+\frac{1}{q}=1$, com $1<p \leq 2$ e $\Omega$ um centralizador real em $X \operatorname{com} \rho(\Omega) \leq \frac{1}{200 q}$, então existem espaços de Köthe $X_{0}$ e $X_{1}$ tais que $X=\left[X_{0}, X_{1}\right]_{1 / 2}$ e se $\Omega_{0}$ é o centralizador induzido em $X$. Então existe uma constante positiva tal que

$$
\left\|\Omega(x)-\Omega_{0}(x)\right\|_{X} \leq C\|x\|_{X}
$$

para todo $x \in X$, ou seja, $\Omega$ e $\Omega_{0}$ são limitadamente equivalentes.

Demonstração. Suponha que $\Omega$ é um centralizador real sobre $X$.

Seja $\Phi^{\Omega}: \mathscr{T}_{\Omega} \longrightarrow \mathbb{R}$ definido por

$$
\Phi^{\Omega}(f)=\int \Omega^{1}(f) d \mu
$$

como na Definição 7.20. A desigualdade (7.9) e a hipóteses do teorema mostram que 


$$
\begin{aligned}
\left|\Phi^{\Omega}(f+g)-\Phi^{\Omega}(f)-\Phi^{\Omega}(g)\right| & \leq 15 \rho\left(\|f\|_{L_{1}}+\|g\|_{L_{1}}\right) \\
& \leq \frac{15}{200 q}\left(\|f\|_{L_{1}}+\|g\|_{L_{1}}\right)
\end{aligned}
$$

$\log 0$

$$
\begin{aligned}
\left|q \Phi^{\Omega}(f+g)-q \Phi^{\Omega}(f)-q \Phi^{\Omega}(g)\right| & \leq \frac{15}{200}\left(\|f\|_{L_{1}}+\|g\|_{L_{1}}\right) \\
& \leq(1-\varepsilon) \log 2\left(\|f\|_{L_{1}}+\|g\|_{L_{1}}\right)
\end{aligned}
$$

onde $\varepsilon=1-\frac{15}{200 \log 2}$. A função $q \Phi^{\Omega}$ é homogênea positiva e verifica as hipóteses do Teorema 6.27 , portanto, existe um espaço de Köthe $W$ e uma constante $c_{1}>0$ tais que

$$
\left|q \Phi^{\Omega}(f)-\left(\Phi_{W}(f)-\Phi_{W^{*}}\right)(f)\right| \leq c_{1}\|f\|_{L_{1}} .
$$

Denotemos por

$$
\Phi_{0}=\Phi_{X}-\frac{1}{2 q}\left(\Phi_{W}-\Phi_{W^{*}}\right) \text { e } \Phi_{1}=\Phi_{X}+\frac{1}{2 q}\left(\Phi_{W}-\Phi_{W^{*}}\right) .
$$

Observe que $\Phi_{0}$ e $\Phi_{1}$ são homogêneas positiva. Agora pelo fato de $\Lambda-\Phi_{W}$ ser convexo, temos que

$$
\begin{aligned}
\Delta_{\Phi_{0}}(f, g) & =\Delta_{\Phi_{X}}(f, g)-\frac{1}{2 q}\left(\Delta_{\Phi_{W}}(f, g)-\Delta_{\Phi_{W^{*}}}(f, g)\right) \\
& =\Delta_{\Phi_{X}}(f, g)-\frac{1}{2 q} \Delta_{\Phi_{W}}(f, g)+\frac{1}{2 q} \Delta_{\Phi_{W^{*}}}(f, g) \\
& \geq \Delta_{\Phi_{X}}(f, g)-\frac{1}{q} \Delta_{\Phi_{W}}(f, g) \\
& \geq \Delta_{\Phi_{X}}(f, g)-\frac{1}{q} \Delta_{\Lambda}(f, g) .
\end{aligned}
$$


Pelo fato de $X$ ser um espaço $p$-convexo, obtemos que $\Lambda-\Phi_{\left(X^{*}\right)^{p}}$ é convexo.

Logo

$$
\begin{aligned}
\Delta_{\Phi_{0}}(f, g) & \geq \Delta_{\Phi_{X}}(f, g)-\frac{1}{q} \Delta_{\Lambda}(f, g) \\
& =\Delta_{\Phi_{X}-\frac{1}{q} \Lambda}(f, g) \\
& =\Delta_{\frac{1}{p} \Lambda-\Phi_{X^{*}}}(f, g) \\
& =\frac{1}{p} \Delta_{\Lambda-\Phi_{\left(X^{*}\right)}}(f, g) \\
& \geq 0,
\end{aligned}
$$

isto mostra que $\Phi_{0}$ é um funcional convexo. Similarmente

$$
\begin{aligned}
\Delta_{\Phi_{0}}(f, g) & =\Delta_{\Phi_{X}}(f, g)-\frac{1}{2 q}\left(\Delta_{\Phi_{W}}(f, g)-\Delta_{\Phi_{W^{*}}}(f, g)\right) \\
& =\Delta_{\Phi_{X}}(f, g)-\frac{1}{2 q} \Delta_{\Phi_{W}}(f, g)+\frac{1}{2 q} \Delta_{\Phi_{W^{*}}}(f, g) \\
& \leq \Delta_{\Phi_{X}}(f, g)+\frac{1}{q} \Delta_{\Phi_{W^{*}}}(f, g) \\
& =\Delta_{\Phi_{X}}(f, g)+\left(1-\frac{1}{p}\right)\left(\Delta_{\Lambda}-\Delta_{\Phi_{W}}\right)(f, g) \\
& \leq \Delta_{\Phi_{X}}(f, g)+\left(1-\frac{1}{p}\right) \Delta_{\Lambda}(f, g) \\
& =\Delta_{\Phi_{X}}(f, g)-\frac{1}{p} \Delta_{\Lambda}(f, g)+\Delta_{\Lambda}(f, g) \\
& \leq \Delta_{\Lambda}(f, g),
\end{aligned}
$$

onde a última desigualdade é justificada pelo fato de ser $X$ um espaço $p$-convexo . Isto prova que $\Phi_{0}-\Lambda$ é uma função convexa. De forma análoga demonstramos que $\Phi_{1}$ e $\Phi_{1}-\Lambda$ são convexos. O Teorema 6.20 implica que existem espaços de Köthe $X_{0}$ e $X_{1}$ tais que $\Phi_{0}=\Phi_{X_{0}}$ e $\Phi_{1}=\Phi_{X_{1}}, \log \mathrm{o}$

$$
\Phi_{X_{0}}+\Phi_{X_{1}}=2 \Phi_{X}
$$


isto é, $X=\left[X_{0}, X_{1}\right]_{1 / 2}$ (Teorema 6.20), com isto demonstramos a primeira parte do teorema. Para demonstrar a segunda parte do teorema note que

$$
\Phi_{X_{1}}-\Phi_{X_{0}}=\frac{1}{q}\left(\Phi_{W}-\Phi_{W^{*}}\right)
$$

A Proposição 7.22 implica que $\Phi^{\Omega_{0}}=\Phi_{X_{1}}-\Phi_{X_{0}}$. Portanto,

$$
\begin{aligned}
\left|\Phi^{\Omega}(f)-\Phi^{\Omega_{0}}(f)\right| & =\left|\Phi^{\Omega}(f)-\frac{1}{q}\left(\Phi_{W}-\Phi_{W^{*}}\right)(f)\right| \\
& \leq \frac{c_{1}}{q}\|f\|_{L_{1}}
\end{aligned}
$$

para todo $f \in \mathscr{T}_{\Omega} \cap \mathscr{T}_{\Omega_{0}}$.

Sejam $x \in X$ e $y \in X^{*}$ tais que $x y \in \mathscr{T}_{\Omega} \cap \mathscr{T}_{\Omega_{0}}$. Por definição, $\Omega^{1}(x y), \Omega_{0}^{1}(x y)$ pertence a $L_{1}$, portanto, $y \Omega^{1}(x), y \Omega_{0}^{1}(x)$ são integráveis. Agora para $y \in X^{*}$, temos que

$$
\begin{aligned}
\left|\int\left(\Omega(x)-\Omega_{0}(x)\right) y d \mu\right| \leq & \left|\int y \Omega(x) d \mu-\int \Omega^{1}(x y) d \mu\right|+\left|\int \Omega^{1}(x y) d \mu-\int \Omega_{0}^{1}(x y) d \mu\right| \\
& +\left|\int \Omega_{0}^{1}(x y) d \mu-\int y \Omega_{0}(x) d \mu\right| \\
& =\left\|y \Omega(x)-\Omega^{1}(x y)\right\|_{L_{1}}+\left|\Phi^{\Omega}(x y)-\Phi^{\Omega_{0}}(x y)\right|+\left\|\Omega_{0}^{1}(x y)-y \Omega_{0}(x)\right\|_{L_{1}} \\
\leq & 5 \rho\|x\|_{X}\|y\|_{X^{*}}+\frac{c_{1}}{q}\|x\|_{X}\|y\|_{X^{*}}+5 \rho_{0}\|x\|_{X}\|y\|_{X^{*}} \\
& \leq C\|x\|_{X}\|y\|_{X^{*}} .
\end{aligned}
$$

por $X^{*}$ ser isométrico a um subespaço normando do dual usual de $X$, obtemos que

$$
\left\|\Omega(x)-\Omega_{0}(x)\right\|_{X} \leq C\|x\|_{X}
$$

para todo $x \in X$. 


\section{Bibliografia}

[1] A.P. CALDERón, Intermediate spaces and interpolation, the complex method, Studia Math. 24 (1964), 113-190.

[2] Alexander E. Litvak, The Extension of the Finite-Dimensional Version of Krivine's Theorem to Quasi-Normed Spaces, Convex Geometric Analysis MSRI Publications vol.34 (1998).

[3] Anton R. Schep, Products of Cesàro convergent sequences with applications to convex solid sets and integral operators, Proc. Amer. Math. Soc., 137 (2009),579-584.

[4] Donald L. Cohn, Measure Theory, Birkhäuser, Boston, USA (2013).

[5] D.H. Fremlin, Measure Theory Vol.II, Torres Fremlin, Colchester, England (2010).

[6] Georgi E. Shilov, Linear algebra, Dover Publications, Inc., New York (1977).

[7] J. Diestel and J.J. UHL, JR, Vector Measures, Mathematical Surveys and Monographs Vol. 15, Amer. Math. Soc. (1977).

[8] J. Bergh, J. Löfström, Interpolation Spaces. An Introduction, Springer Verlag, Berlin (1976).

[9] J.M.F. Castillo and M. Gonzalez, Three-space Problems in Banach Space Theory, Lecture Notes in Math. 1667, Springer, Berlin (1997).

[10] J. KomLós, A generalization of a problem of Steinhaus, Acta Math. Hungar. 18 (1967) 217-229.

[11] J. Lindenstrauss, L. Tzafriri, Classical Banach Spaces I and II, Function Spaces, Springer, Berlin (1979).

[12] M. Fabian, P. Habala, P. Hájek, V. Montesinos Santalucía, J. Pelant, V. Zizler, Functional Analysis and Infinite-Dimensional Geometry, Springer-Verlag, New York (2001). 


\section{BIBLIOGRAFIA}

[13] G. PIsIER, Some applications of the complex interpolation method to Banach lattice, Journal d'Analyse Mathématique vol.5 (1979), 264-281.

[14] Micheál Ó Searcoíd, Metric Spaces, Springer Undergraduate Mathematics Series, London (2006).

[15] N.J Kalton, Analytic functions in non-locally convex spaces, Studia Math. 83 (1986), No.3, 275-303.

[16] N.J Kalton, Differentials of complex interpolation processes for Köthe function spaces, Trans. Amer. Math. Soc. 333 (1992), 479-529.

[17] N.J Kalton, Nonlinear commutators in interpolation theory, Mem. Amer. Math. Soc. No.385 (1988).

[18] N.J Kalton, Convexity, type and the three-space problem, Studia Math. 69 (1980), 57-96.

[19] N.J Kalton, N.T Peck, Twisted sums of sequence spaces and three space problem, Trans. Amer. Math. Soc. 255 (1979), 1-30.

[20] Peter Meyer-Nieberg, Banach Lattices, Universitext, Springer-Verlag, Berlin (1991)

[21] P. Sam Johnson, S. BalajI, On Linear Operators with Closed Range, Journal of Applied Mathematics and Bioinformatics (2011) vol.1, No.2, 175-182.

[22] Robert E. Megginson, An introduction to Banach space theory Springer-Verlag, New York Berlin Heidelberg (1998).

[23] Serge Lang, Complex analysis, Vol. 106 Graduate Texts in Mathematics. Springer-Verlag, fourth edition, New York (1999).

[24] S. Okada,W.J. Ricker, E. A. Sanchez, Optimal Domain and Integral Extension of Operators, Acting in Function Spaces, Oper. Theory Adv. Appl., vol. 180 Birkhäuser, Basel (2008).

[25] T.A. GillespIE, Factorization in Banach function spaces,Indag. Math. 43 (1981), 287-300.

[26] Tакаsнi Iто, On Banach spaces with unique isometric predual, Michigan Math. J.(1977) Vol.24, 321-324.

[27] W. Arendt,Charles J.K. Batty, M. Hieber,F. Neubrander Vector-valued Laplace Transforms and Cauchy Problems: Second Edition, Birkhäuser, Natick, MA (2001)

[28] W. Rudin, Real and Complex Analysis, McGraw-Hill (1970).

[29] W.B. Johnson, J. Lindenstrauss, Handbook of the Geometry of Banach spaces, Vol.2 eds, Elsevier, Amsterdam (2003), 1099-1130. 